\title{
Turtle Anoxia - Biochemistry and Gene Regulation in an Anaerobic Extremist
}

\author{
Anastasia Krivoruchko \\ B.Sc. Carleton University, 2006
}

\begin{abstract}
A Thesis Submitted to the Faculty of Graduate Studies and Research in partial fulfillment of the requirements for the degree of
\end{abstract}

\author{
Doctor of Philosophy \\ Department of Biology
}

\author{
Carleton University \\ Ottawa, Ontario, Canada
}

(C) copyright 2010

Anastasia Krivoruchko 


$\begin{array}{ll}\begin{array}{l}\text { Library and Archives } \\ \text { Canada }\end{array} & \begin{array}{l}\text { Bibliothèque et } \\ \text { Archives Canada }\end{array} \\ \begin{array}{l}\text { Published Heritage } \\ \text { Branch }\end{array} & \begin{array}{l}\text { Direction du } \\ \text { Patrimoine de l'édition }\end{array} \\ \begin{array}{l}\text { 395 Wellington Street } \\ \text { Ottawa ON K1A ON4 }\end{array} & \begin{array}{l}395, \text { rue Wellington } \\ \text { Ottawa ON K1A ON4 } \\ \text { Canada }\end{array} \\ \text { Canada }\end{array}$

Your file Votre référence

ISBN: 978-0-494-70526-1

Our file Notre référence

ISBN: $978-0-494-70526-1$

NOTICE:

The author has granted a nonexclusive license allowing Library and Archives Canada to reproduce, publish, archive, preserve, conserve, communicate to the public by telecommunication or on the Internet, loan, distribute and sell theses worldwide, for commercial or noncommercial purposes, in microform, paper, electronic and/or any other formats.

The author retains copyright ownership and moral rights in this thesis. Neither the thesis nor substantial extracts from it may be printed or otherwise reproduced without the author's permission.
AVIS:

L'auteur a accordé une licence non exclusive permettant à la Bibliothèque et Archives Canada de reproduire, publier, archiver, sauvegarder, conserver, transmettre au public par télécommunication ou par l'internet, prêter, distribuer et vendre des thèses partout dans le monde, à des fins commerciales ou autres, sur support microforme, papier, électronique et/ou autres formats.

L'auteur conserve la propriété du droit d'auteur et des droits moraux qui protège cette thèse. $\mathrm{Ni}$ la thèse ni des extraits substantiels de celle-ci ne doivent être imprimés ou autrement reproduits sans son autorisation.
In compliance with the Canadian Privacy Act some supporting forms may have been removed from this thesis.

While these forms may be included in the document page count, their removal does not represent any loss of content from the thesis.
Conformément à la loi canadienne sur la protection de la vie privée, quelques formulaires secondaires ont été enlevés de cette thèse.

Bien que ces formulaires aient inclus dans la pagination, il n'y aura aucun contenu manquant. 


\begin{abstract}
While the physiological responses to oxygen deprivation have been studied extensively in the anoxia-tolerant turtle, Trachemys scripta elegans, adaptations of transcriptional regulatory processes are mostly unknown. This thesis addresses this knowledge gap by examining the anoxia responsiveness of several important proteins and pathways in T. s. elegans tissues. The unfolded protein response (UPR) was activated in turtle heart, kidney and liver, as evidenced by increased phosphorylation of PERK and increased expression and activation of ATF4. Enhanced expression of the molecular chaperones GRP78 and GRP94, as well as other UPR-responsive proteins was also observed. These results suggest that the UPR is an important component of stress

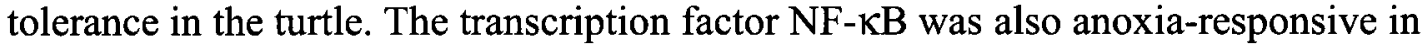
turtle liver and activated via increased expression of its component proteins, increased nuclear presence and increased DNA-binding activity. Transcript levels of NF- $\kappa \mathrm{B}$ target genes involved in antioxidant defense and anti-apoptotic signaling were also upregulated under anoxia. The FoxO transcription factors, implicated in hypometabolism and stress resistance, were also anoxia-responsive. Studies of FoxO expression, phosphorylation status, nuclear presence and DNA-binding activity showed that FoxO1 and FoxO3 were both activated in liver, whereas FoxO3 was activated in heart and kidney. FoxO target genes involved in cell cycle arrest and stress resistance were also upregulated in liver under anoxia. Expression and activation of the transcriptional inhibitors, histone deacetylases (HDACs), was strongly elevated in white skeletal muscle during anoxia, with a lesser response in liver cells, results that indicated an important role for HDACs in anoxia-mediated transcriptional suppression. Finally, the metabolic transcription factor
\end{abstract}


involved in control of glycolytic enzymes, ChREBP, was activated in the liver in response to $5 \mathrm{~h}$ of anoxia, and its target gene, LPK, was transcriptionaly induced, suggesting a role for this transcription factor in adjusting carbohydrate metabolism for anaerobiosis. Overall, the data in this thesis enhance understanding of the gene and protein adaptations that support cellular endurance of anoxia and document several new mechanisms that are involved in stress resistance, hypometabolism and fuel metabolism as being key to natural anoxia tolerance. 


\section{ACKNOWLEDGEMENTS}

First and foremost, I would like to thank my thesis supervisor, Dr. Kenneth Storey, for taking me into his lab when I was still an undergrad and turning me into a scientist. I am grateful for his scientific and non-scientific advice, for giving me the opportunity to try new things and for shaping me into the best scientist I can be. I am also grateful for all the chocolate he provided me with over the years. In addition, I am extremely grateful to Jan Storey for her excellent editing, comments and suggestions of my manuscripts, talks and posters, as well as her vigorous editing of my thesis.

I would also like to thank my committee members, Dr. Katie Gilmour and Dr. John Vierula, for their support and valuable suggestions. Thanks also goes to all the storey lab members, past and present, for their help, support, and role in making this such an amazing lab.

Finally, I would like to thank my parents for always pushing me to succeed in my younger years, as well as their continuous support and encouragement throughout my graduate studies. 


\section{TABLE OF CONTENTS}

Title Page

Acceptance Sheet

$\begin{array}{ll}\text { Abstract } & \text { iii }\end{array}$

Acknowledgements $\quad$ V

Table of Contents vi

List of Abbreviations vii

List of Figures $\quad$ xi

List of Tables $\quad$ xvi

List of Appendices $\quad$ xvi

Chapter 1 General Introduction 1

Chapter 2 Activation of the unfolded protein response upon anoxic 22 submergence in Trachemys scripta elegans.

Chapter 3 Molecular mechanisms of anoxia tolerance: a role for NF- $\quad 78$ $\kappa \mathrm{B}$.

Chapter 4 Activation of the FoxO transcription factors in response to $\quad 110$ anoxia in the turtle Trachemys scripta elegans.

Chapter 5 Epigenetics in anoxia tolerance: a role for histone 155 deacetylases

Chapter 6 Changing metabolism: activation of ChREBP in response 188 to anoxia

$\begin{array}{llr}\text { Chapter } 7 & \text { General Discussion } & 213\end{array}$

$\begin{array}{ll}\text { Appendices } & 238\end{array}$

$\begin{array}{ll}\text { References } & 261\end{array}$ 


\section{LIST OF ABBREVIATIONS}

AA

AMP, ADP, ATP

AMPK

APS

ATF

$\mathrm{Bcl}$

BiP

BLAST

bZip

cAMP

CDK

cDNA

CHOP

ChRE

ChREBP

CRE

CREB

CTBP

$\mathrm{Cu} / \mathrm{Zn}$ SOD

DAF

DEPC

dNTP

DTT amino acid

adenosine mono-, di-, or triphosphate

AMP activated protein kinase

ammonium persulphate

activating transcription factor

B-cell leukemia/lymphoma

binding immunoglobulin protein

Basic Local Alignment Search Tool

basic leucine zipper

3', 5'-cyclic adenosine monophosphate

cyclin dependent kinase

complementary deoxyribonucleic acid

CCAAT/enhance-binding protein (C/EBP) homologous protein

carbohydrate response element

carbohydrate response element binding protein

cAMP response element

cAMP response element binding protein

C-terminal binding protein

Copper/Zinc superoxide dismutase

dauer formation

diethylpyrocarbonate

deoxynucleotide triphosphate

dithiolthreitol 


\begin{tabular}{|c|c|}
\hline E2F & E2 promoter binding factor \\
\hline ECL & enhanced chemiluminescence \\
\hline EDEM & ER degradation-enhancing alpha-mannosidase-like protein \\
\hline EDTA & ethylenediamine tetraacetic acid \\
\hline $\operatorname{eIF} 2 \alpha$ & eukaryotic initiation factor $2 \alpha$ subunit \\
\hline ELISA & enzyme-linked immunosorbent assay \\
\hline ER & endoplasmic reticulum \\
\hline ERAD & ER associated degradation \\
\hline EtBr & ethidium bromide \\
\hline ETC & electron transport chain \\
\hline FoxO & forkhead box, class $O$ protein \\
\hline FTH1 & ferritin heavy chain 1 \\
\hline G6Pase & glucose-6-phosphatase \\
\hline GADD & growth arrest and DNA damage gene \\
\hline GPX & glutathione peroxidase \\
\hline GR & glutathione reducatse \\
\hline GRP & glucose regulated protein \\
\hline GSH & reduced glutathione \\
\hline GSSG & oxidized glutathione \\
\hline GST & glutathione $S$-transferase \\
\hline GTP & guanosine triphosphate \\
\hline HDAC & histone deacetylase \\
\hline HEPES & N- (2-hydroxyethyl) piperazine-N'- (2-ethanesulfonic acid) \\
\hline HIF-1 & hypoxia-inducible factor- 1 \\
\hline HO-1 & heme oxygenase-1 \\
\hline
\end{tabular}




\begin{tabular}{|c|c|}
\hline HP1 & heterochromatin protein 1 \\
\hline HRP & horseradish peroxidase \\
\hline HSF1 & heat shock factor 1 \\
\hline HSP & heat shock protein \\
\hline IRE1 & inositol requiring kinase 1 \\
\hline $\mathbf{I} \mathbf{B}$ & inhibitor of $\mathrm{NF} \kappa \mathrm{B}$ \\
\hline JNK & jun-N-terminal kinase \\
\hline $\mathbf{k b}$ & kilobase \\
\hline $\mathrm{kDa}$ & kilodalton \\
\hline Keap1 & kelch-like ECH-associated protein 1 \\
\hline LPK & liver pyruvate kinase \\
\hline MnSOD & manganese superoxide dismutase \\
\hline mRNA & messenger RNA \\
\hline MST1 & mammalian Sterile 20-like kinase 1 \\
\hline $\mathrm{Na}^{+} \mathrm{K}^{+}$-ATPase & ATP-dependent sodium potassium pump \\
\hline NCBI & National Center for Biotechnology Information \\
\hline $\mathrm{NF}-\kappa \mathrm{B}$ & nuclear factor kappa B \\
\hline Nrf2 & nuclear factor-erythroid 2 related factor -2 \\
\hline ORF & open reading frame \\
\hline PAGE & polyacrylamide gel electrophoresis \\
\hline PBS & phosphate-buffered saline \\
\hline PCR & polymerase chain reaction \\
\hline PDI & protein disulfide isomerase \\
\hline PDK1 & phosphoinositide-dependent kinase \\
\hline PEP & phosphoenolpyruvate \\
\hline
\end{tabular}




\begin{tabular}{|c|c|}
\hline PEPCK & phosphoenolpyruvate carboxykinase \\
\hline PERK & PKR-like endoplasmic reticulum kinase \\
\hline PGC & PPAR $\gamma$ co-activator \\
\hline PI3K & phosphoinositide 3-kinase \\
\hline PIP3 & phosphatidyl inositol-(3,4,5)-triphosphate \\
\hline PKA & cAMP- activated protein kinase \\
\hline PMSF & phenylmethanesulfonyl fluoride \\
\hline PP2A & protein phosphatase $2 \mathrm{~A}$ \\
\hline PVDF & polyvinylidine fluoride \\
\hline RACE & rapid amplification of cDNA ends \\
\hline $\mathrm{Rb}$ & retinoblastoma protein \\
\hline ROS & reactive oxygen species \\
\hline RT & room temperature $\left(\sim 21^{\circ} \mathrm{C}\right)$ \\
\hline RT-PCR & reverse-transcriptase polymerase chain reaction \\
\hline SCP & sterol carrier protein \\
\hline SDS & sodium dodecyl sulfate \\
\hline STAT3 & signal transducer and activator of transcription 3 \\
\hline TAE & tris-acetate-ethylenediamine tetraacetic acid buffer \\
\hline TBST & tris-buffered saline containing Tween-20 \\
\hline TEMED & $\mathrm{N}, \mathrm{N}, \mathrm{N}^{\prime}, \mathrm{N}^{\prime}$-tetra methylethylenediamine \\
\hline TMB & 3,3',5,5'-Tetramethylbenzidine \\
\hline Tris & tris (hydroxymethyl) aminomethane \\
\hline UPR & unfolded protein response \\
\hline $\mathrm{X} 5 \mathrm{P}$ & xylulose-5-phosphate \\
\hline XBP1 & $\mathrm{X}$-box binding protein \\
\hline
\end{tabular}




\section{LIST OF FIGURES}

FIG.1.1 Duration of anoxic submergence at various temperatures from

which painted turtles (Chrysemys picta bellii) are known to recover fully.

FIG.1.2 ATP levels in turtles. The figure indicates that a coordinated decrease occurs in both ATP production and ATP utilization when a turtle makes the transition from a normoxic to an anoxic state.

FIG.2.1 Overview of the Unfolded Protein Response (UPR).

FIG.2.2 Effect of 5 and $20 \mathrm{~h}$ of anoxic submergence on the phosphorylation $\quad 59$ status of (A) PERK and (B) eIF $2 \alpha$ in T. s. elegans tissues.

FIG.2.3 Complete cDNA sequence of atf 4 amplified from liver of $T$. $s$. elegans and the translated amino acid sequence from three open reading frames.

FIG.2.4 Complete amino acid sequence of ATF4 from T. s. elegans aligned with ATF4 from other vertebrates

FIG.2.5 (A) Homology tree of turtle ATF4 protein compared to other vertebrates. (B) Homology matrix showing amino acid identity of the $T$. s. elegans ATF4 sequence compared with ATF4 from the other vertebrates.

FIG.2.6 Effect of 5 and $20 \mathrm{~h}$ of anoxic submergence on ATF4 (A) transcript and (B) protein expression in $T$. s. elegans tissues.

FIG.2.7 Effect of 5 and $20 \mathrm{~h}$ of anoxic submergence on ATF4 (A) nuclear levels and (B) DNA-binding activity in $T$. s. elegans tissues.

FIG.2.8 (A) A partial cDNA sequence of atf3 with the corresponding amino acid sequence. (B) Homology tree produced from an alignment of the partial T. s. elegans ATF3 protein sequence with ATF3 from other vertebrates.

FIG.2.9 Effect of 5 and $20 \mathrm{~h}$ of anoxic submergence on ATF3 (A) transcript and (B) protein expression in $T$. s. elegans tissues.

FIG.2.10 Effect of 5 and $20 \mathrm{~h}$ of anoxic submergence on the protein expression of GADD34 in T. s. elegans tissues. 
FIG.2.11 (A) A partial cDNA sequence of $g r p 78$ is shown with the corresponding amino acid sequence. (B) Homology tree produced from an alignment of the partial $T$. s. elegans GRP78 protein sequence with GRP78 from other vertebrates.

FIG.2.12 Effect of 5 and $20 \mathrm{~h}$ of anoxic submergence on GRP78 (A) transcript and (B) protein expression in $T$. s. elegans tissues.

FIG.2.13 (A) A partial cDNA sequence of $g r p 94$ is shown with the 75 corresponding amino acid sequence. (B) Homology tree produced from an alignment of the partial $T$. s. elegans GRP94 protein sequence with GRP94 from other vertebrates.

FIG.2.14 Effect of 5 and $20 \mathrm{~h}$ of anoxic submergence on GRP94 (A) transcript 77 and (B) protein expression in $T$. s. elegans tissues.

FIG.3.1 Activation of NF- $\mathrm{KB}$ in response to anoxia.

FIG.3.2 Effect of $5 \mathrm{~h}$ of anoxic submergence on levels of phosphorylated $\mathrm{I} \kappa \mathrm{B} \alpha(\mathrm{Ser} 32)$ in tissues of T.s. elegans.

FIG.3.3 Nucleotide and deduced amino acid sequence for T. s. elegans partial NFKB1 sequence.

FIG.3.4 Nucleotide and deduced amino acid sequence for T. s. elegans partial p65 sequence.

FIG.3.5 Effect of 5 and $20 \mathrm{~h}$ of anoxic submergence on transcript levels of the 100 proteins making up the NF- $\mathrm{kB}$ dimer, NFKB1 (p50) and $\mathrm{p} 65$ in $T$. $s$. elegans liver.

FIG.3.6 Effect of 5 and $20 \mathrm{~h}$ of anoxic submergence on the protein levels of 101 the proteins making up the NF-KB dimer, p50 and p65 in $T . s$. elegans liver.

FIG.3.7 Effect of 5 and $20 \mathrm{~h}$ of anoxic submergence on nuclear protein levels 102 of the proteins making up NF- $\mathrm{kB}$ dimer, p50 and p65 in T. s. elegans liver.

FIG.3.8 Effect of 5 and $20 \mathrm{~h}$ of anoxic submergence on the DNA-binding 103 activity of NF-KB in $T$. s. elegans liver.

FIG.3.9 Nucleotide and deduced amino acid sequence for T. s. elegans partial 104 Fth1 sequence 
FIG.3.10 Nucleotide and deduced amino acid sequence for T. s. elegans partial 105 $M n S O D$ sequence.

FIG.3.11 Nucleotide and deduced amino acid sequence for T. s. elegans partial 106 $\mathrm{Cu} / \mathrm{ZnSOD}$ sequence.

FIG.3.12 Effect of 5 and $20 \mathrm{~h}$ of anoxic submergence on transcript levels of antioxidant target genes of NF- $\mathrm{KB}$ in $T$. s. elegans liver.

FIG.3.13 Nucleotide and deduced amino acid sequence for $T$. s. elegans partial 108 $B c l 2$ sequence.

FIG.3.14 Effect of 5 and $20 \mathrm{~h}$ of anoxic submergence on transcript levels of 109 pro-survival target genes of NF-KB in T. s. elegans liver.

FIG.4.1 An overview of the FoxO pathway.

FIG.4.2 (A) The partial cDNA sequence of foxo1, with the corresponding amino acid sequence. (B) Homology tree produced from an alignment of the partial T. s. elegans FoxOl protein sequence with FoxO1 from other vertebrates.

FIG.4.3 The partial amino acid sequence of FoxO1 from T. s. elegans compared with FoxO1 sequences from other vertebrates.

FIG.4.4 Effect of 5 and $20 \mathrm{~h}$ anoxic submergence on foxo1 transcript expression in T. s. elegans tissues as determined by RT-PCR.

FIG.4.5 Effect of 5 and $20 \mathrm{~h}$ anoxic submergence on the protein expression of (A) FoxO1 and (B) phospho-FoxO1 (Ser256) as determined by immunobloting.

FIG.4.6 Effect of 5 and $20 \mathrm{~h}$ anoxic submergence on FoxO1 (A) nuclear levels and (B) DNA-binding activity in $T$. s. elegans tissues.

FIG.4.7 (A) The partial cDNA sequence of foxo3, with the corresponding amino acid sequence. (B) Homology tree produced from an alignment of the partial $T$. s. elegans FoxO3 protein sequence with FoxO3 from other vertebrates.

FIG.4.8 The partial amino acid sequence of $\mathrm{FoxO} 3$ from $T$. s. elegans compared with FoxO3 sequences from other vertebrates.

FIG.4.9 Effect of 5 and $20 \mathrm{~h}$ anoxic submergence on foxo 3 transcript expression in $T$. s. elegans tissues as determined by RT-PCR. 
FIG.4.10 Effect of 5 and $20 \mathrm{~h}$ anoxic submergence on the protein expression of 146 (A) FoxO3, (B) p-FoxO3 (253) and (C) p-FoxO3 (ser318/21).

FIG.4.11 Effect of 5 and $20 \mathrm{~h}$ anoxic submergence on FoxO3 (A) nuclear levels and (B) DNA-binding activity in T. s. elegans tissues.

FIG.4.12 (A) The partial cDNA sequence of $p 27 k i p 1$, with its corresponding amino acid sequence. (B) Homology tree produced from an alignment of the partial $T$. s. elegans $\mathrm{p} 27 \mathrm{kip} 1$ protein sequence with p27kip1 from other vertebrates.

FIG.4.13 Effect of 5 and $20 \mathrm{~h}$ anoxic submergence on p27Kipl transcript expression in $T$. s. elegans tissues as determined by RT-PCR.

FIG.4.14 (A) The partial cDNA sequence of catalase, with the corresponding 152 amino acid. (B) Homology tree produced from an alignment of the partial $T$. s. elegans catalase protein sequence with catalase from other vertebrates.

FIG.4.15 Effect of 5 and $20 \mathrm{~h}$ anoxic submergence on catalase transcript 154 expression in T. s. elegans tissues as determined by RT-PCR.

FIG.5.1 Nucleotide and deduced amino acid sequence for T. s. elegans partial 175 Hdacl sequence.

FIG.5.2 Nucleotide and deduced amino acid sequence for T. s. elegans partial 176 Hdac2 sequence.

FIG.5.3 Nucleotide and deduced amino acid sequence for T. s. elegans partial Hdac3 sequence.

FIG.5.4 Nucleotide and deduced amino acid sequence for T. s. elegans partial 178 Hdac4 sequence.

FIG.5.5 Nucleotide and deduced amino acid sequence for $T$. s. elegans partial Hdac5 sequence.

FIG.5.6 Effect of 5 and $20 \mathrm{~h}$ of anoxic submergence on transcript levels of Hdac1-5 in T. s. elegans heart.

FIG.5.7 Effect of 5 and $20 \mathrm{~h}$ of anoxic submergence on protein levels of (A) class I and (B) class II HDACs in T. s. elegans heart.

FIG.5.8 Effect of 5 and $20 \mathrm{~h}$ of anoxic submergence on transcript levels of Hdac1-5 in T. s. elegans liver. 
FIG.5.9 Effect of 5 and $20 \mathrm{~h}$ of anoxic submergence on protein levels of (A)

class I and (B) class II HDACs in T. s. elegans liver.

FIG.5.10 Effect of 5 and $20 \mathrm{~h}$ of anoxic submergence on transcript levels of 184 Hdac1-5 in T. s. elegans muscle.

FIG.5.11 Effect of 5 and $20 \mathrm{~h}$ of anoxic submergence on protein levels of (A) class I and (B) class II HDACs in T. s. elegans muscle

185

FIG.5.12 Effect of 5 and $20 \mathrm{~h}$ of anoxic submergence on HDAC activity in $T$. s. elegans muscle.

FIG.5.13 Effect of 5 and $20 \mathrm{~h}$ of anoxic submergence on total and acetylated 187 (Lys 9 and 23) histone $\mathrm{H} 3$ expression in the heart, liver, and white skeletal muscle of $T$. s. elegans.

FIG.6.1 A model for ChREBP activation.

FIG.6.2 Nucleotide and deduced amino acid sequence for T. s. elegans partial 206 ChREBP sequence.

FIG.6.3 The partial amino acid sequence of ChREBP from $T$. s. elegans aligned with ChREBP sequences from other vertebrates.

FIG.6.4 (A) Homology tree produced from an alignment of the T. s. elegans 208 ChREBP protein sequence with ChREBP from other vertebrates. (B) Homology matrix showing amino acid identity of the $T$. s. elegans ChREBP sequence compared with ChREBP from the other vertebrates.

FIG.6.5 Effect of 5 and $20 \mathrm{~h}$ of anoxic submergence on ChREBP (A) transcript and (B) protein expression in $T$. $s$. elegans kidney and liver.

FIG.6.6 Effect of $5 \mathrm{~h}$ anoxic submergence on subcellular distribution of 210 ChREBP.

FIG.6.7 Effect of $5 \mathrm{~h}$ anoxic submergence on the DNA-binding activity of ChREBP in nuclei isolated from kidney and liver.

FIG.6.8 Effect of 5 and $20 \mathrm{~h}$ anoxic submergence on $L P K$ transcript expression in $T$. s. elegans liver as determined by RT-PCR.

FIG.7.1 Overview of cross-talk between the different pathways examined in this thesis. 
FIG.7.2 Overview of the molecular responses observed in turtle liver in response to anoxia.

\section{LIST OF TABLES}

TABLE.1.1 The main ATP-demanding pathways during normoxia and anoxia in turtle hepatocytes.

TABLE.2.1 List of primers used in chapter 2 .

TABLE.3.1 List of primers used in chapter 3.

TABLE.4.1 List of primers used in chapter 4 .

TABLE.5.1 List of primers used in chapter 5.

\section{LIST OF APPENDICES}

$\begin{array}{lll}\text { Appendix A } & \text { List of publications } & 239\end{array}$

$\begin{array}{lll}\text { Appendix B } & \text { Communications at scientific meetings } & 240\end{array}$

$\begin{array}{lll}\text { Appendix C } \quad \text { List of GenBank submissions } & 242\end{array}$

$\begin{array}{lll}\text { Appendix D } \quad \text { Extra amino acid alignments } & 243\end{array}$

Appendix E Ensuring antibody specificity for western blotting 259

Appendix F $\quad$ Examples of constantly expressed proteins in T. s. elegans 260 tissues 


\section{CHAPTER 1}

\section{GENERAL INTRODUCTION}




\section{Oxygen and oxygen depletion}

Some two billion years ago primitive life forms evolved to use oxygen as the final acceptor in their electron transport pathways. The high redox potential of oxygen allows it to easily accept electrons from reduced substrates, making it extremely useful for energy production. The ability to extract greater amounts of energy from organic molecules by using oxygen-linked catabolism has driven life to evolve into high complexities and has made oxygen vital to many life forms on Earth. Many animals are sensitive to oxygen deprivation and situations of hypoxia or anoxia can lead rapidly to severe tissue damage or even death.

Decrease in the oxygen supply leads to inhibition of oxidative phosphorylation and subsequent interruption in mitochondrial ATP production. This leads to a disruption of many ATP-utilizing processes in the cell. Loss of function of ATP-dependent ion channels can be particularly damaging since it disrupts the normal balance between the opposing rates of ATP-dependent ion pumps versus ATP-independent ion channels and results in a quick loss of membrane potential difference (Hochachka and Lutz, 2001; Storey, 2007). Loss of membrane potential difference causes a rapid breakdown of critical transmembrane ion gradients, a rise in intracellular $\mathrm{Ca}^{2+}$ concentrations and a release of excitatory neurotransmitters, which triggers multiple dangerous effects on physiological functions including nerve transmission and muscle contraction (Fraser et al., 2001).

Many animals experience situations of oxygen deprivation that occur either due to variations in environmental oxygen levels or behaviors that interrupt oxygen supply. As a result, most animals have developed mechanisms that allow them to compensate for mild 
or short-term hypoxia. In mammals, these responses are activated in order to (1) improve oxygen delivery to tissues and (2) increase anaerobic ATP production to compensate for the reduced ATP output. Physiological responses include increases in ventilation and heart rates, an increase in hemoglobin unloading of oxygen, and release of stored red blood cells from the spleen. These adaptations serve to increase $\mathrm{O}_{2}$ uptake, carrying capacity, and delivery to organs (Storey, 2004a). Biochemical responses to oxygen deprivation include an increase in the glycolytic rate, as well as consumption of creatine phosphate reserves (in tissues that have large phosphagen pools) (Storey, 2004a). However, despite these responses, severe oxygen deprivation can still be extremely damaging.

\section{Physiological adaptations to anoxia}

In contrast, some ectothermic vertebrates are particularly well-adapted to surviving oxygen limitation. Various species of turtles have very well-developed capacities for living without oxygen (called anaerobiosis) (Storey, 1996; Storey, 2007). For example, freshwater turtles living in northern regions of the United States and Canada typically hibernate under water to elude freezing temperatures on land. However, bodies of water often become ice-locked, limiting the ability of animals to surface for lung breathing. Some turtle species compensate for this situation with a good capacity for extrapulmonary (aquatic) gas exchange across other epithelia whereas others have perfected strategies that allow survival without oxygen for three months or more (Ultsch, 2006). Sliders and painted turtles, for example, can survive without oxygen for up to two weeks at $16-18^{\circ} \mathrm{C}$ and for $12-18$ weeks at $3^{\circ} \mathrm{C}$ (FIG.1.1; Ultsch, 2006). Those species 
that tolerate extreme hypoxia or anoxia employ biochemical and physiological mechanisms for survival, the major ones including prehibernation accumulation of huge glycogen stores to fuel anaerobic glycolysis, the use of the shell and skeletal systems for buffering lactate accumulation and minimizing acidosis, and a capacity for profound metabolic rate depression to only $10-20 \%$ of the corresponding aerobic rate. It also appears that a number of gene expression mechanisms are involved in turtle anoxia tolerance (discussed later), but these have received relatively little attention to date.

\section{Anoxia and oxidative stress}

In addition to the challenges presented to tissues by severe oxygen limitation, turtles must also deal with the potential for oxidative stress that occurs with the reintroduction of oxygen. The mitochondrial electron transport chain becomes reduced during hypoxia/anoxia because there is little or no oxygen to accept electrons. This condition makes it particularly susceptible to generating reactive oxygen species (ROS) upon reoxygenation when the oxygen tension is high. This can be extremely damaging to

cells and indeed, in situations of ischemia-reperfusion, mammalian tissues show profound damage caused by ROS during the recovery phase (Churchill, 2004; Ma et al., 2008). Situations of environmental oxygen variability, such as those experienced by diving or hibernating turtles, could present many opportunities for oxidative stress and oxidative damage, and yet, turtles emerge apparently unharmed from repeated cycles of anoxia and reoxygenation.

Formation of most ROS occurs at the site where the vast majority of intracellular oxygen is consumed, the mitochondria (Boveris and Chance, 1973). It is here that 
reduced substrates donate their electrons to form an electrochemical gradient, resulting ultimately in the production of ATP, the energy currency of the cell. This occurs through a series of oxidative reactions conducted by protein complexes on the inner membrane of the mitochondria. Leakage of electrons from these complexes results in the one-electron reduction of oxygen to form superoxide, the precursor to most ROS. It is estimated that approximately $1-2 \%$ of the oxygen consumed during respiration is reduced to superoxide (Cadenas et al., 1977). Dismutation of superoxide results in the production of hydrogen peroxide, whereas the subsequent iron (or copper) catalyzed breakdown of $\mathrm{H}_{2} \mathrm{O}_{2}$ (Fenton reaction) generates hydroxyl radicals (Hermes-Lima, 2004). Hydroxyl radicals are highly reactive and are responsible for most of the damage incurred to biological macromolecules by ROS. Because of their short half life (less than $1 \mathrm{~ns}$ ), hydroxyl radicals attack molecules very close to their site of formation (Valko et al., 2004) and their very high reactivity makes them virtually impossible to scavenge (Halliwell, 2007). The reaction of hydroxyl radicals with biomacromolecules produces new radicals, usually with lower reactivity. Once unleashed upon the intracellular milieu, ROS react with many cellular macromolecules, including lipids, proteins and DNA, inflicting damaging effects on multiple subcellular structures.

\section{Antioxidant defenses and anoxia tolerance}

In order to avoid oxidative-stress-related damage during anoxia-reoxygenation bouts, anoxia-tolerant organisms generally make use of two strategies: the maintenance of constitutively high antioxidant defenses and anoxia-induced antioxidant defenses.

Maintenance of high levels of antioxidant defenses can prevent oxidative damage 
from occurring during successive bouts of anoxia and recovery. For example, the redeared slider turtle, Trachemys scripta elegans maintains high constitutive activities of various antioxidant enzymes including catalase, superoxide dismutase (SOD) and alkyl hydroperoxide reductase (Willmore and Storey, 1997a). These activities are much higher than those in other ectothermic vertebrates and are actually comparable to mammalian activities even though the metabolic rate of turtles is much lower than mammalian values. Tissue pools of glutathione are also higher in turtle organs compared to other ectotherms (Willmore and Storey, 1997b), and ascorbic acid levels in turtle brain cortex are 2-3 times higher than in mammals (Rice et al., 1995).

The structure of turtle antioxidant enzymes might also be especially suitable for frequent anoxic excursions. For example, turtle glutathione reductase (GR), an enzyme that catalyzes the reduction of oxidized glutathione (GSSG), has a higher affinity for GSSG than the enzyme in most other vertebrates (Willmore and Storey, 2007), allowing for better enzyme efficiency under different redox conditions. Turtle GR also showed high activity over a broad $\mathrm{pH}$ range including at the low $\mathrm{pH}$ values typical of anoxic conditions (Willmore and Storey, 2007). In another recent study, two alpha classes of glutathione $S$-transferases (GSTs) were found in $T$. s. elegans liver, a homodimer and a heterodimer (Willmore and Storey, 2005). Both exhibited a major reduction in specific activity in response to anoxia and the heterodimer showed a major change in substrate preference, indicating an anoxia-responsive stable modification of the enzyme (Willmore and Storey, 2005). Therefore, it appears that GST undergoes modifications in response to changing oxygen availability to optimize its function.

These mechanisms could help the turtle avoid oxidative damage during situations 
of oxygen variability. Indeed, it was shown that the ratio of GSH/GSSG, which decreases under oxidative stress, actually increases during recovery from anoxia exposure in turtles (compared to control) suggesting that no oxidative stress occurs during reoxygenation (Willmore and Storey, 1997b). In addition, oxidative damage products were largely unaffected over the course of anoxia/recovery in turtle organs, as evident from minimal changes in lipid peroxidation products (Willmore and Storey, 1997b).

In addition to the maintenance of constitutively-high antioxidant defenses, anticipatory changes in antioxidant defenses during the anoxic period have been observed for several species, including anoxia tolerant garter snakes (Hermes-Lima and Storey 1993), estivating land snails (Hermes-Lima and Storey 1995a) and anoxia tolerant carp (Vig and Nemcsok 1989). Induction of proteins associated with antioxidant defense has also been observed in turtle organs in response to anoxia. Heme oxygenase-1 (HO-1) is an enzyme that might have antioxidant functions during anoxia/reoxygenation episodes. It is involved in the degradation of heme, a major source of redox active iron in the cell, and its induction is also typically tied to the simultaneous upregulation of ferritin, which sequesters the iron released by the heme degradation reaction (Soares and Bach, 2009; Vile et al., 1994). HO-1 was upregulated in response to anoxia in turtle brain (Kesaraju et al., 2009), which might be a demonstration of the importance of minimizing redoxactive iron during anoxia. In mammals, the expression of HO-1 is known to be induced in response to various forms of oxidative stress (Motterlini et al., 1996; Doi et al., 1999; Motterlini et al., 2000) and its expression is believed to be protective in different disease states (Abraham and Kappas, 2008). The important antioxidant properties of HO-1 also stem from biliverdin, one of the products of heme catabolism by HO-1. Biliverdin and its 
catabolite, bilirubin, have antioxidant properties with demonstrated beneficial effects in a number of disease/injury states including ischemia-reperfusion, organ transplantation, graft rejection, and autoimmune inflammation (Soares and Bach, 2009; Ollinger et al., 2007; Idriss et al., 2008).

The transcript levels of several other antioxidant enzymes have been shown to increase in response to anoxia in the turtle. Array screening showed that several antioxidant genes were up-regulated by anoxia exposure in heart and liver of hatchling painted turtles Chrysemys picta marginata: SOD-1, glutathione peroxidase (GPX) isozymes 1 and 4, GST isozymes M5 and A2, and peroxiredoxin 1 (Storey, 2006). The activity of the antioxidant enzyme, $\gamma$-glutamyltranspeptidase, also increased significantly during freezing (an anoxic/ischemic stress) in liver of painted turtles (Hemmings and Storey, 2000), whereas catalase activity increased under anoxia exposure in livers of several hatchling turtle species (Dinkelacker et al., 2005). In addition, the protein levels of the transcription factor Nrf2 and several downstream antioxidant enzymes were significantly elevated in the brains of $C . p$. marginata hatchlings in response to freezing exposure (Krivoruchko and Storey, 2010a).

\section{Other molecular adaptations to anoxia and reoxygenation stress}

Proteins that are particularly sensitive to changes in intracellular $\mathrm{pH}$, redox state and ion milieu, conditions that occur in cells during anoxia and reoxygenation, can lose their native folded conformation to become misfolded. Proliferation of heat shock proteins (HSPs) is one of the best known cytoprotective mechanisms in response to stress (Lindquist and Craig, 1988). HSPs act as molecular chaperones, helping to fold nascent 
proteins, as well as aiding in the refolding of misfolded proteins under stress conditions (Lindquist and Craig, 1988; Kalmar and Greensmith, 2009). By their chaperone action, HSPs help to preserve cellular proteins and extend their functional life. HSPs also play a protective role in a number of pathological conditions associated with oxidative stress, including ischemia, cardiovascular disease and neurodegeneration (Kalmar and Greensmith, 2009).

Studies have found significantly higher levels of Hsp60 in the hearts of anoxiatolerant painted turtles compared to anoxia intolerant softshell turtles, rabbits and rats (Chang et al., 2000). Hsp60 is a predominantly mitochondrial chaperone involved in the folding of proteins entering the mitochondria. Hsp60 also has protective effects against oxidative stress (Hollander et al., 2003). High constitutive levels of Hsp72 have also been reported in turtle brain (Prentice et al., 2004). Hsp72 is known as the inducible HSP70 family member, and is generally found at very low levels under normal conditions. The constitutive expression of $\mathrm{Hsp} 72$ in turtle brain suggests that it has some importance in neuroprotection in this animal.

As in the case of antioxidant proteins, HSPs and other molecular chaperones also show inducibility in response to anoxia in turtle tissues. These include Hsp25/27, Hsp40, Hsp60, Hsp72, Hsc70, and Hsp90 (Kesaraju et al., 2009; Prentice et al., 2004;

Ramaglia and Buck, 2004; Krivoruchko and Storey, 2010b). Array screening of turtle brain comparing aerobic and anoxic animals also showed upregulation of transcripts coding for $\alpha \mathrm{B}$-crystallin, another known chaperone (Storey, 2007). In addition, the heat shock transcription factor (HSF1) that is responsible for the activation of the heat shock response, was activated in response to anoxia in turtle tissues (Krivoruchko and Storey, 
2010b). Activation of the heat shock response in response to anoxia might help maintain protein stability under anoxic conditions as well as serve as a preparative mechanism for re-oxygenation, since increased HSP expression might also actively prevent damage following oxidative stress.

\section{Anoxia tolerance and metabolic rearrangements}

When animals face a decrease in their oxygen supply, they can raise anaerobic ATP production to meet energy demands, suppress ATP needs to lower their energy demands via metabolic rate depression, or use a combination of both strategies. Increasing the rate of glycolysis does increase ATP output, but also results in a quick depletion of internal fuel reserves as well as a large accumulation of end products. Some species of fish have dealt with that by accumulating very large glycogen reserves to fuel glycolysis and converting lactate to ethanol that can be easily excreted. However, metabolic rate depression still appears to be the preferred strategy utilized by the majority of facultative anaerobes to survive anoxia. Indeed, in turtles, a profound metabolic rate depression to $10-20 \%$ of the corresponding aerobic resting rate at the same temperature occurs in response to anoxia. This suppresses, rebalances and reprioritizes the rates of ATP-utilizing and ATP-generating processes so that turtles can sustain long term viability without oxygen. FIG.1.2 shows that both ATP production and ATP consumption are strongly decreased in response to anoxia in turtles. Entrance into hypometabolism is not just an anoxia-specific phenomenon, but is a common survival strategy across phylogeny and is observed in hibernation, torpor, aestivation, anaerobiosis, diapause, and anhydrobiosis. 
Several factors can contribute to metabolic rate depression in turtles, including reduced voluntary movement by skeletal muscles, reducing heart, breathing and kidney filtration rates, and energy savings associated with reduced/halted digestion and absorption if animals do not eat while hibernating in cold water (Storey, 2004a). In addition, since the metabolic rate of ectotherms directly correlates with the external temperature, many ectotherms seek cooler habitats to decrease their body temperatures and reduce oxygen demands under hypoxic or anoxic conditions (Tattersall and Boutilier, 1997).

Multiple intrinsic factors are also involved in reducing cellular energy demands. Entrance into hypometabolism in response to anoxia in turtles is characterized by a reorganization of energy-consuming processes. For example, studies with turtle hepatocytes showed a $94 \%$ decrease in overall ATP turnover under anoxia, but found that five main ATP-consuming processes were suppressed to different degrees (Table 1.1; Hochachka et al., 1996). Under anoxia, the $\mathrm{Na}^{+} \mathrm{K}^{+}$-ATPase became the dominant energy consumer of the cell, consuming $62 \%$ of total ATP turnover as compared with $28 \%$ in normoxia. Meanwhile, protein synthesis and degradation were strongly suppressed by $>90 \%$, whereas gluconeogenesis and urea synthesis were virtually halted. Similar reductions in protein synthesis were also observed in other organs (Fraser et al., 2001). This reorganization of metabolic processes allows turtle cells to direct energy into the most vital processes for cellular survival under anoxia and results in the most efficient ATP utilization under energy-limited conditions. 
Reversible protein phosphorylation

The depression of metabolic rate in response to anoxia, as well as the reorganization of metabolic priorities is largely accomplished by reversible protein phosphorylation of cellular enzymes and functional proteins. Reversible protein phosphorylation is considered to be the most widespread and powerful mechanism utilized by cells to make stable changes to enzyme activities in response to various stimuli (Storey and Storey, 2004, 2007). This mechanism also plays an important role in the regulation of metabolic depression in many other hypometabolic systems (Storey and Storey, 2004, 2007). Reversible protein phosphorylation regulates the activities and kinetic properties of enzymes involved in diverse cellular processes, including membrane receptors and transporters, ion channels and ATPases, proteins involved in gene transcription, protein synthesis and degradation, cell cycle control and signal transduction cascades. For example, it was shown that reversible phosphorylation is involved in the regulation of voltage-gated ion channels $\left(\mathrm{Na}^{+}, \mathrm{Ca}^{2+}, \mathrm{K}^{+}\right)$and membrane receptors (e.g. $N$ methyl-D-aspartate-type glutamate receptor) in anoxia-tolerant turtles (Hochachka and Lutz, 2001; Bickler et al., 2001; Bickler and Buck, 2007). In addition, changes in the phosphorylation status of various ribosomal translation factors were observed in turtle tissues in response to anoxia (Rider et al., 2009), suggesting that such changes are involved in anoxia-induced suppression of protein synthesis in turtles.

\section{OBJECTIVES AND HYPOTHESES}

Understanding the molecular nature of vertebrate anoxia tolerance is important not only from a comparative perspective, but also has applications for medical science. 
Whereas mammalian tissues have a very limited tolerance for oxygen deprivation, an anoxia-tolerant vertebrate would be expected to activate responses that are beneficial for hypoxic/anoxic survival, thereby highlighting the processes that help an animal to cope with oxygen deprivation. One aspect of natural anoxia tolerance that has received very little attention to date is the role of gene expression. Therefore, it is of particular interest to further examine the changes in gene and protein expression associated with anoxia tolerance and the regulatory factors that control these. In my thesis, I chose to take a gene-based approach to analyzing the response to anoxia exposure by red-eared slider turtles, T. s. elegans, with a focus on three areas that appear to be crucial for anoxia tolerance:

1. Stress-responsive pathways

2. Metabolic rate depression

3. Metabolic rearrangements

To gain a better understanding of the adaptive changes occurring in response to anoxia, these mechanisms were examined in multiple tissues of $T$. s. elegans by comparing the response to $5 \mathrm{~h}$ of anoxic submergence, which represents the hypoxic transition stage, as well as $20 \mathrm{~h}$ of anoxia, a time by which anoxia-induced metabolic rate depression is well developed.

\section{Objective 1: activation of stress-responsive pathways}

As mentioned above, bouts of anoxia and reoxygenation can be extremely harmful for organisms. In addition to stresses caused by oxygen (and energy) depletion, anoxia is also characterized by reduced $\mathrm{pH}$ (due to lactate accumulation) and altered redox conditions. Activation of stress-responsive pathways could help to preserve cell 
viability during anaerobiosis. Indeed, as mentioned above, activation of the heat shock response is one major component of anoxia tolerance in turtles. However, little is known about other stress-responsive pathways that could contribute to anoxia tolerance. In this thesis I examine two additional stress-responsive pathways, the unfolded protein response and the NF- $\kappa B$ pathway, to evaluate their potential role in anoxia tolerance.

Hypothesis 1: Activation of stress-responsive cytoprotective pathways and transcription factors, including the unfolded protein response and the NF- $\mathrm{KB}$ pathway, will support anoxia survival in T. s. elegans.

Chapter 2 tests this hypothesis by exploring the activation of the unfolded protein response in turtle organs in response to anoxia. The unfolded protein response (UPR) is a pivotal cytoprotective pathway that becomes activated in response to accumulation of unfolded proteins in the endoplasmic reticulum. This response involves several pathways whose collective goal is to relieve protein folding stress by reducing protein synthesis, degrading slowly folding or permanently unfolded proteins, and increasing the folding capacity of the cell. As previously mentioned, anoxia can compromise protein folding and therefore, activation of the unfolded protein response could help alleviate any folding stress occurring in the endoplasmic reticulum under anoxic conditions.

Chapter 2 examines the responses of multiple UPR-regulated proteins to anoxia in T. s. elegans tissues. The phosphorylation status of two important components of the response, PERK and eIF $2 \alpha$ are examined. In addition, activation of the stress-activated transcription factor ATF4 is analyzed by measurement of its transcript and protein levels, nuclear localization and DNA-binding activity, as well as upregulation of its target genes. 
The expression of two key UPR-inducible molecular chaperones, GRP78 and GRP94, is also examined.

Chapter 3 tests the hypothesis by examining the activation of NF- $\kappa B$ in response to anoxia in the turtle. NF- $\mathrm{KB}$ is an oxygen-responsive transcription factor, known to be activated in response to a variety of stimuli and to control the expression of hundreds of target genes, including genes involved in the immune response, stress response, antioxidant defenses, cell growth and differentiation, and apoptosis. Because of its ability to activate a variety of genes involved in cytoprotection, especially those involved in antioxidant defense and anti-apoptosis, activation of NF- $\mathrm{KB}$ in response to anoxia could be beneficial to T. s. elegans tissues.

Initial experiments suggested that liver is the primary organ demonstrating NF- $\mathrm{kB}$ activation under anoxic conditions, so studies focused on this tissue. Transcript and protein levels of NF- $\mathrm{KB}$, as well as nuclear localization and DNA-binding activity were examined. In addition, the expression patterns of NF- $\mathrm{kB}$ target genes involved in antioxidant defense and anti-apoptosis were examined.

\section{Objective 2: Activation of pathways involved in hypometabolism}

The suppression of metabolic rate is a crucial aspect of anoxia tolerance, as it allows a lowering of cellular energy demands to a level that can be met by ATP generation via anaerobic glycolysis alone and greatly extends the time that fixed tissue stores of carbohydrates can sustain viability. However, outside of reversible protein phosphorylation, little is known about the molecular regulatory mechanisms involved in 
anoxia-responsive metabolic rate depression in T. s. elegans.

Hypothesis 2: Anoxic survival by T. s. elegans will include activation of mechanisms that promote hypometabolism, including activation of FoxO transcription factors and histone deacetylases.

Chapter 4 tests this hypothesis by examining the anoxia-responsiveness of the forkhead class $\mathrm{O}$ (FoxO) transcription factors. FoxOs are evolutionarily conserved transcription factors that appear to have a key role in hypometabolism through promotion of cell cycle arrest. In addition, FoxO activation promotes resistance to oxidative stress. Therefore, activation of FoxOs could be a physiologically-relevant mechanism of anoxia tolerance in T. s. elegans.

Chapter 4 examines the gene and protein expression of two proteins belonging to the FoxO family, FoxO1 and FoxO3, as well as their phosphorylation status, nuclear localization, and DNA-binding activity. In addition, the status of several FoxO target genes are assessed by analyzing their transcript levels; these include genes involved in metabolic depression and antioxidant defense.

Chapter 5 further tests the hypothesis by examining the role of histone deacetylases in anoxia tolerance. Histone acetylation is associated with higher transcriptional activity because this modification makes chromatin more accessible to proteins of the transcriptional apparatus. Histone deacetylases (HDACs) are a group of enzymes that catalyze the removal of acetyl groups from histones, and as such are associated with transcriptional repression. Because transcription has a large metabolic 
cost, it must be repressed in situations of hypometabolism and activation of HDACs could potentially address this need.

Chapter 5 examines the expression of HDACs in three tissues, analyzing changes in Hdac transcript and HDAC protein levels in response to anoxia. In addition, HDAC activity in turtle muscle was measured under aerobic and anoxic conditions.

\section{Objective 3: Metabolic rearrangement}

Under normal conditions, organisms rely mainly on carbohydrate and lipid fuels for energy production. However, upon oxygen deprivation, lipid fuels become useless because they can only be catabolized in an oxygen-dependent manner in mitochondria. To deal with this metabolic reality, anoxia-exposed turtles must readjust metabolic pathways to redirect carbon flux into glycolytic pathways in response to anoxia. The transcription factor ChREBP (Carbohydrate Element Binding Protein) plays important roles in carbohydrate metabolism in the cell. It is activated in response to high glucose concentrations and induces genes involved in glycolysis (such as pyruvate kinase) and lipogenesis. Because of its key role in regulating liver pyruvate kinase (LPK), activation of ChREBP in response to anoxia could be beneficial as it could enhance glycolytic capacity in response to oxygen limitation, allowing cells to meet their ATP demands, particularly within the hypoxia transition period before metabolic depression sets in.

Hypothesis 3: ChREBP is activated in response to anoxia in T. s. elegans, resulting in upregulation of its glycolytic target gene, LPK.

Chapter 6 studies ChREBP activation by examining its transcript and protein 
expression, nuclear localization, and DNA-binding activity, as well as the transcript levels of its target gene LPK in T. s. elegans kidney and liver in response to anoxia. 
FIG.1.1

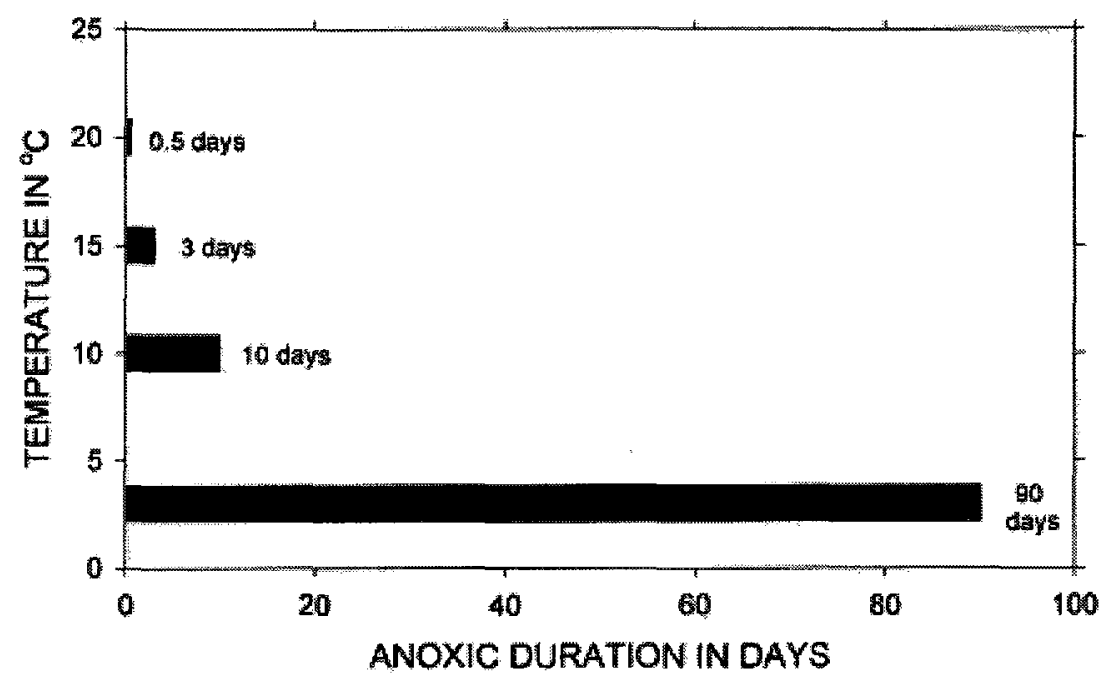

FIG.1.1 Duration of anoxic submergence at various temperatures from which painted turtles (Chrysemys picta bellii) are known to recover fully. Data are from Jackson (2000) (adapted from Herbert and Jackson, 1985). 
FIG.1.2

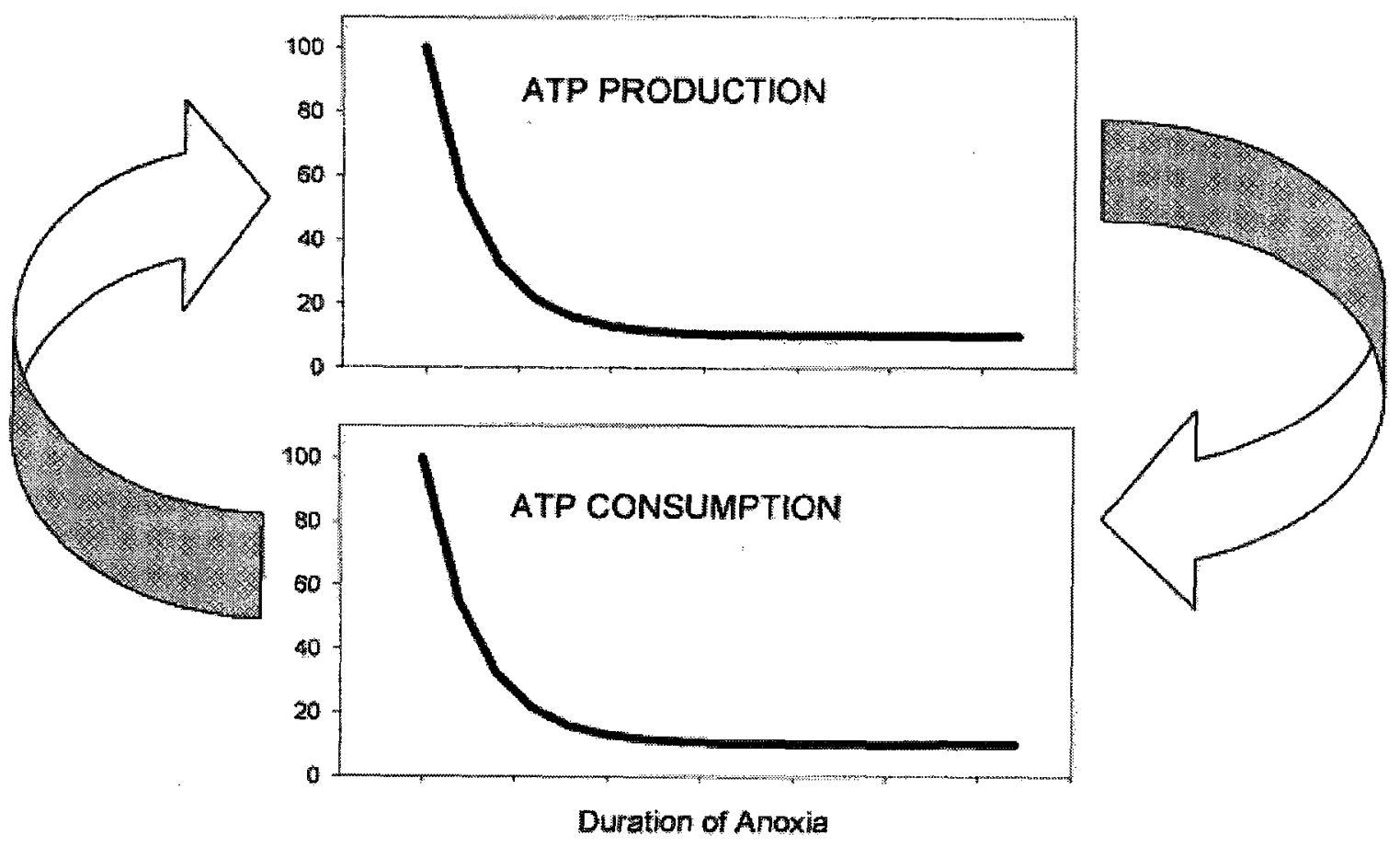

FIG.1.2. ATP levels in turtles. The figure indicates that a coordinated decrease occurs in both ATP production and ATP utilization when a turtle makes the transition from a normoxic to an anoxic state. Modified from Jackson (2000). 
TABLE 1.1. The main ATP-demanding pathways during normoxia and anoxia in turtle hepatocytes. (adapted from Hochachka et. al., 1996).

\begin{tabular}{lccc}
\hline & \multicolumn{3}{c}{ ATP demand, $\mu$ mol ATP $\times \mathrm{g}^{-1} \times \mathrm{h}^{-1}$} \\
\cline { 2 - 4 } \multicolumn{1}{c}{ Pathway } & Normoxia & Anoxia & \% suppression \\
\hline Total & 67.0 & 6.3 & 94 \\
$\mathrm{Na}^{+}$pump & 19.1 & 4.8 & 75 \\
Protein synthesis & 24.4 & 1.6 & 93 \\
Protein breakdown & 11.1 & 0.7 & 94 \\
Urea synthesis & 2.0 & 0.6 & 70 \\
Gluconeogenesis & 11.4 & 0.0 & 100 \\
\hline
\end{tabular}




\section{CHAPTER 2 \\ Activation of the unfolded protein response upon anoxic submergence in T. s. elegans}

(Publication in preparation) 


\subsection{INTRODUCTION}

The endoplasmic reticulum (ER) of eukaryotic cells is the first compartment of the secretory pathway. It is the site of membrane and secretory protein synthesis, as well as the site where the majority of secretory and transmembrane proteins fold into their native conformations (Schroder and Kaufman, 2005a,b). It is also the site where nascent proteins undergo several post-translational modifications, including $\mathrm{N}$-linked glycosylation (Ruddock and Moliani, 2006) and disulfide bond formation (Ellgaard and Ruddock, 2005). Conditions that interfere with ER functions are termed ER stress. Various stress conditions can cause perturbations in the protein folding of the ER, leading to accumulation of unfolded proteins in the ER lumen and ER stress. This leads to the activation of the unfolded protein response (UPR) (reviewed by Schroder, 2008; Schroder and Kaufman, 2005a,b). The UPR consists of several signaling pathways that act to return the ER to its normal physiological state (FIG. 2.1) (Selye, 1985). This response is evolutionarily conserved from yeast to man and is known to be activated in response to various stressors.

Initiation of the UPR is controlled by three ER-resident protein sensors: the two protein kinases IRE1 (inositol requiring kinase 1) (Sidrauski and Walter, 1997) and PERK (double-stranded RNA-activated protein kinase-like ER kinase (Harding et al., 2000) and the transcription factor ATF6 (activating transcription factor 6) (Yoshida et al., 2000). It is believed that under normal conditions, these proteins are found in an inactive state through association with the ER-resident chaperone GRP78 (BiP) (Watowich et al., 1991; Bertolotti et al., 2000). Accumulation of unfolded proteins leads GRP78 to interact with these proteins and decreases its association with the luminal 
domains of the sensors (Bertolotti et al., 2000). This decrease in association, in turn, leads to activation of the sensors and initiation of the UPR that triggers a series of protective measures designated to restore the cell to homeostasis, including inhibition of global protein translation to attenuate the influx of proteins into the ER (Harding et al., 1999; Shi et al., 1999; Shi et al., 1998), induction of chaperone proteins to increase the protein-folding capacity of the ER (Kozutsumi et al., 1988; Dorner et al., 1992), and induction of genes involved in the ER-associated degradation pathway to remove proteins that cannot be refolded (Friedlander et al., 2000; Travers et al., 2000; Oda et al., 2006). If the accumulation of unfolded proteins in the ER is not resolved following activation of the UPR, the UPR can also induce apoptosis.

PERK is an ER-associated transmembrane serine/threonine protein kinase with a cytoplasmic activity that is selectively activated by ER stress (Shi et al., 1998; Harding et al., 1999). Upon accumulation of unfolded proteins in the ER lumen, PERK dimerizes and autophosphorylates, leading to its activation (Harding et al., 2000). It then phosphorylates a key ribosomal protein, the eukaryotic initiation factor 2 on its alpha subunit (eIF2 $\alpha$ ) at Ser 51 (Harding et al., 1999; Shi et al., 1998). Because eIF2 $\alpha$ transports the initiating methionine residue to the assembling ribosome to start protein synthesis, phosphorylation and inactivation of this protein leads to a rapid attenuation of protein synthesis in the cell and is the most immediate response to ER stress (Kaufman, 2004).

In addition to translational attenuation, activation of PERK induces many UPRdependent genes (Scheuner et al., 2001; Harding et al., 2003; Harding et al., 1999; Ron, 2002). Its downstream transcription factor, ATF4, is an important stress-responsive 
transcription factor that is activated upon PERK-mediated phosphorylation of eIF2 $\alpha$ (Scheuner et al., 2001; Harding et al., 2003; Harding et al., 1999; Ron, 2002). ATF4 activates the transcription of several genes involved in amino acid biosynthesis and transport, antioxidant stress responses, and apoptosis (Scheuner et al., 2001; Harding et al., 2003; Harding et al., 1999; Ron, 2002). Because eIF2 $\alpha$ can be phosphorylated by several different kinases in response to stress stimuli, ATF4 translation is stimulated by different stress inducers and serves as a common downstream response to integrate these signaling events (Rutkowski and Kaufman, 2003; Ron, 2002).

Translation inhibition by PERK is generally transient in order to allow recovery from ER stress as well as permit an efficient protein synthesis response to prolonged ER stress. GADD34 is a UPR-responsive gene whose expression is induced by ATF4 late in ER stress (Ma and Hendershot, 2003). It can bind to the catalytic subunit of protein phosphatase 1 (PP1), and direct it toward eIF $2 \alpha$, promoting the dephosphorylation of eIF2 $\alpha$ (Connor et al., 2001; Brush et al., 2003). Another target of ATF4 is activating transcription factor 3 (ATF3). Recently it has been suggested that ATF3 promotes a proapoptotic function during nutritional stress, but a pro-survival function during ER stress, the difference in roles being attributed to different binding partners of ATF3 (Jiang et al., 2004).

Various molecular chaperones lie downstream of UPR activation and are induced to increase the folding capacity of the ER. The best described are the glucose-regulated proteins GRP78 and GRP94 (Schröder, 2008). These proteins reside in the ER and are involved in the folding and assembly of newly synthesized proteins. Induction of these 
genes has been used extensively as a marker for the onset of the UPR (Lee, 2001).

I hypothesized that the UPR will be activated in response to anoxia in turtle tissues to preserve and stabilize cellular proteins over the anoxic episode. Anoxia is characterized by altered redox conditions and decreased $\mathrm{pH}$, which could disrupt protein folding. In addition, many of the protein foldases operating in the ER require an oxidizing environment and molecular oxygen as their final electron acceptor and could therefore be severely compromised during oxygen deprivation. Previous studies have demonstrated increased production of heat shock proteins and other molecular chaperones in response to anoxia (Prentice et al., 2004; Krivoruchko and Storey, 2010b). Like the heat shock response, activation of the UPR could help relieve cellular stress associated with anoxia and restore cells to homeostasis. The present chapter explores the responses of multiple UPR-regulated proteins in response to anoxia. These include ATF4, ATF3, GADD34, GRP78 and GRP94 as well as the phosphorylated forms of PERK and-eIF2 $\alpha$.

\subsection{MATERIALS AND METHODS}

\subsubsection{Animals}

Adult red eared slider turtles, $T$. s. elegans, were purchased from Carolina Biological (Burlington, NC, USA). All turtles (aerobic control turtles and anoxic turtles) were handled exactly the same. Turtles had been previously held at $\sim 11^{\circ} \mathrm{C}$ and were initially placed at this temperature in a holding tank containing dechlorinated water. Temperature was then lowered to $\sim 4^{\circ} \mathrm{C}$ over one week and animals were then acclimated to $4^{\circ} \mathrm{C} \pm 1{ }^{\circ} \mathrm{C}$. Aerobic control turtles were sampled from this condition. Animals were 
euthanized by decapitation, and then tissue samples were quickly dissected and immediately frozen in liquid nitrogen. To impose anoxia, other turtles were moved singly into containers filled with dechlorinated water at $4^{\circ} \mathrm{C}$ that had been previously bubbled with $100 \%$ extra dry nitrogen gas for at least $6 \mathrm{~h}$ beforehand. After turtles were placed in a tank, a wire mesh was fitted $\sim 10 \mathrm{~cm}$ below the water line so that turtles could not surface during the anoxic episode; a low level of nitrogen bubbling continued throughout. Animals were exposed to 5 or $20 \mathrm{~h}$ of anoxia and were then rapidly sampled. Tissues obtained included the heart, kidney, liver and white skeletal muscle (from the neck). All turtles survived the experimental treatments. Animals were cared for in accordance with the guidelines of the Canadian Council on Animal Care and all experimental procedures had the prior approval of the Carleton University Animal Care Committee.

\subsubsection{RNA extraction}

All solutions and materials were treated with $0.1 \%$ diethylpyrocarbonate (DEPC) and autoclaved prior to use. Total RNA was isolated from liver tissue of normoxic, $5 \mathrm{~h}$ and $20 \mathrm{~h}$ anoxic turtles using Trizol ${ }^{\mathrm{TM}}$ (Invitrogen). Briefly, $100 \mathrm{mg}$ of tissue was homogenized in $1 \mathrm{~mL}$ Trizol using a Polytron homogenizer followed by the addition of $200 \mu \mathrm{L}$ of chloroform and centrifugation at $10,000 \times g$ for $15 \mathrm{~min}$ at $4^{\circ} \mathrm{C}$. The upper aqueous phase (containing the RNA) was removed to a fresh tube and precipitated by the addition of $500 \mu \mathrm{L}$ isopropanol followed by incubation for $10 \mathrm{~min}$ at room temperature (RT). The samples were then centrifuged again at $10,000 \times g$ for $15 \mathrm{~min}$ at $4^{\circ} \mathrm{C}$. The total RNA pellet was washed with $1 \mathrm{~mL}$ of $70 \%$ ethanol and centrifuged again as above. The 
resulting pellet was air-dried for $10-15 \mathrm{~min}$ and resuspended in $50 \mu \mathrm{L}$ of DEPC-treated water. The quality of RNA was judged based on the ratio of absorbances at 260 and $280 \mathrm{~nm}$ as well as gel electrophoresis on a 1\% agarose gel stained with ethidium bromide to check for the integrity of $18 \mathrm{~S}$ and $28 \mathrm{~S}$ ribosomal RNA bands.

\subsubsection{Primers}

Primers were synthesized by Sigma Genosys. Forward and reverse primers for all genes were designed based on conserved regions of these genes as determined from a DNA alignment of the sequences from other vertebrate species including human (Homo sapiens), chicken (Gallus gallus), western clawed frog (Xenopus tropicalis), and zebrafish (Danio rerio). For grp94 and grp78, perfect (species-specific) primers were designed based on the turtle sequence and then used for quantification. The primers and their conditions are listed in table 2.1.

\subsection{4. cDNA synthesis and PCR}

A $4 \mu \mathrm{g}$ aliquot of RNA was diluted with DEPC water to $10 \mu \mathrm{L}$ final volume and used to synthesize cDNA. Briefly, $1 \mu \mathrm{L}$ of $200 \mathrm{ng} / \mu \mathrm{L}$ Oligo $d T$ (5'TTTTTTTTTTTTTTTTTTTTTTV-3') primer was added to the RNA sample and incubated at $65^{\circ} \mathrm{C}$ for $5 \mathrm{~min}$. The mixture was then chilled rapidly on ice and $4 \mu \mathrm{L} 5 \mathrm{X}$ first strand buffer, $2 \mu \mathrm{L} 100 \mathrm{mM}$ DTT, $1 \mu \mathrm{L}$ dNTP mixture (10 mM each) and $1 \mu \mathrm{L}$ reverse transcriptase enzyme M-MLV (all reagents from Invitrogen) were added for a 
total volume $19 \mu \mathrm{L}$. The mix was incubated at $42^{\circ} \mathrm{C}$ for $1 \mathrm{~h}$ and chilled to $4^{\circ} \mathrm{C}$. The resulting cDNA was diluted $\left(10^{-1}\right.$ to $\left.10^{-3}\right)$ and amplified by polymerase chain reaction (PCR) with the forward and reverse primers mentioned above. The PCR reaction of $25 \mu \mathrm{L}$ final volume was composed by mixing $13.25 \mu \mathrm{L}$ of sterile water, $5 \mu \mathrm{L}$ of diluted cDNA, $1.25 \mu \mathrm{L}$ of primer mixture (to a final concentration of $1.5 \mu \mathrm{M}$ forward and $1.5 \mu \mathrm{M}$ reverse), $2.5 \mu \mathrm{L}$ of $10 \times$ PCR buffer (Invitrogen), $1.5 \mu \mathrm{L}$ of $50 \mathrm{mM} \mathrm{MgCl}_{2}, 0.5 \mu \mathrm{L}$ of dNTP mixture ( $10 \mathrm{mM}$ each) and $1 \mu \mathrm{L}$ of Taq Polymerase. The PCR program was as follows: $7 \mathrm{~min}$ at $94^{\circ} \mathrm{C}$ followed by 36 cycles of $1 \mathrm{~min}$ at $94^{\circ} \mathrm{C}, 1 \mathrm{~min}$ at a predetermined annealing temperature $\left(54^{\circ} \mathrm{C}\right.$ for atf 3 and tubulin, $62.5^{\circ} \mathrm{C}$ for $a t f 4, \operatorname{grp} 78$ and $\left.g r p 94\right)$, and $1.5 \mathrm{~min}$ at $72^{\circ} \mathrm{C}$. The final step was $72^{\circ} \mathrm{C}$ for $10 \mathrm{~min}$. PCR products were separated on a $1 \%$ agarose gel stained with ethidium bromide, visualized using the ChemiGenius imaging system (Syngene, Frederick, MD, USA) under UV light and quantified using the GeneTools program. The bands from the most dilute cDNA sample that gave visible product were used for quantification purposes to make sure that the products had not reached amplification saturation. The brightness of the bands increased linearly with the dilutions used, so that the lower the dilution, the brighter the band was. In addition, the number of cycles used in all PCR reactions was carefully optimized to ensure linearity. A higher number of cycles to what was used resulted in a significantly brighter (more saturated) band, while a lower number of cycles resulted in the band's disappearance.

\subsubsection{Amplification of $3^{\prime}$ ' and 5 ' ends}

In order to determine whether ATF4 mRNA translation in the turtle is regulated in 
the same manner as in other vertebrates, RACE was used to obtain the 3' and 5' upstream regions. To amplify the 3' end of atf4, $4 \mu \mathrm{g}$ aliquot of RNA was diluted with DEPC water to a $10 \mu \mathrm{L}$ final volume and used to synthesize cDNA. A $1 \mu \mathrm{L}$ aliquot of $10 \mu \mathrm{M}$ AP primer (5' - GGCCACGCGTCGACTAGTACTTTTTTTTTTTTTTTTT - $\left.3^{\prime}\right)$ was added to the RNA sample and incubated at $65^{\circ} \mathrm{C}$ for $5 \mathrm{~min}$. The mixture was then chilled rapidly on ice and $4 \mu \mathrm{L} 5 \mathrm{X}$ first strand buffer, $2 \mu \mathrm{L} 100 \mathrm{mM}$ DTT, $1 \mu \mathrm{L}$ dNTP mixture (10 mM each) and $1 \mu \mathrm{L}$ reverse transcriptase enzyme M-MLV (all reagents from Invitrogen) were added for a total volume $19 \mu \mathrm{L}$. The mix was incubated at $42^{\circ} \mathrm{C}$ for $1 \mathrm{~h}$ and the reaction was terminated by incubating at $70^{\circ} \mathrm{C}$ for $15 \mathrm{~min}$ and chilling to $4^{\circ} \mathrm{C}$. The resulting cDNA was diluted $10^{-1}$ and then amplified using PCR. The PCR reaction of $25 \mu \mathrm{L}$ final volume was composed by mixing $12.5 \mu \mathrm{L}$ of sterile water, $5 \mu \mathrm{L}$ of diluted cDNA, $1 \mu \mathrm{L}$ of $10 \mu \mathrm{M}$ atf 4 forward primer, $1 \mu \mathrm{L}$ of $10 \mu \mathrm{M}$ AP primer, $2.5 \mu \mathrm{L}$ of $10 \times$ PCR buffer (Invitrogen), $1.5 \mu \mathrm{L}$ of $50 \mathrm{mM} \mathrm{MgCl}_{2}, 0.5 \mu \mathrm{L}$ of dNTP mixture (10 mM each) and $1 \mu \mathrm{L}$ of $\mathrm{Taq}$ Polymerase. The PCR program was as follows: 7 min at $94^{\circ} \mathrm{C}$ followed by 36 cycles of $1 \mathrm{~min}$ at $94^{\circ} \mathrm{C}, 1 \mathrm{~min}$ at $68{ }^{\circ} \mathrm{C}$, and $1.5 \mathrm{~min}$ at $72^{\circ} \mathrm{C}$. The product was $\sim 1000$ bp in size.

The 5' end of atf4 was amplified using First Choice RLM RACE kit (Ambion) using the protocol from the manufacturer. The outer primer used was the atf4 reverse primer, whereas the inner primer was 5'-GCATCCTCCTGGCTGTTATCTGTGTT-3'. The annealing temperature was $62.5^{\circ} \mathrm{C}$. The product obtained was $\sim 500$ bp in size. 


\subsubsection{Sequencing}

PCR products were sequenced by DNA Landmarks (St.-Jean-sur-Richelieu, QC). Sequences were verified as encoding the correct genes using the program BLASTN at the NIH (http://www.ncbi.nlm.nih.gov/blast). The sequences were translated using DNAMAN. Each protein sequence was aligned with the respective sequences from other vertebrates, and homology trees were generated based on that alignment using DNAMAN.

\subsubsection{Protein homogenates and western blotting}

Frozen tissue samples were crushed under liquid nitrogen and then homogenized 1:2.5 w:v in homogenizing buffer ( $20 \mathrm{mM}$ Hepes, $\mathrm{pH} 7.5,200 \mathrm{mM} \mathrm{NaCl}, 0.1 \mathrm{mM}$ EDTA, $10 \mathrm{mM} \mathrm{NaF}, 1 \mathrm{mM} \mathrm{Na}_{3} \mathrm{VO}_{4}, 10 \mathrm{mM} \beta$-glycerophosphate) with a few crystals of PMSF and $1 \mu \mathrm{L}$ protease inhibitor cocktail added (Sigma-Aldrich, Oakville, ON, CA). Samples were centrifuged at $4^{\circ} \mathrm{C}$ for $15 \mathrm{~min}$ at $10,000 \times \mathrm{g}$ and the supernatant was collected. Soluble protein concentration was measured using the Coomassie Blue dyebinding method with a prepared reagent (Bio-Rad, Hercules, CA, USA) and bovine serum albumin as the standard. Concentrations were adjusted to $10 \mu \mathrm{g} / \mu \mathrm{L}$ by the further addition of small amounts of homogenizing buffer and then samples were mixed 1:1 v:v with $2 \times$ SDS buffer $(100 \mathrm{mM}$ Tris-base, $4 \% \mathrm{w} / \mathrm{v}$ SDS, $20 \% \mathrm{v} / \mathrm{v}$ glycerol, $0.2 \% \mathrm{w} / \mathrm{v}$ bromophenol blue, $10 \% \mathrm{v} / \mathrm{v} 2$-mercaptoethanol) to give a final protein concentration of $5 \mu \mathrm{g} / \mu \mathrm{L}$. Samples were boiled for $5 \mathrm{~min}$ and chilled on ice prior to loading onto gels. Aliquots of control and anoxic samples containing $30 \mu \mathrm{g}$ protein each were loaded 
onto $10 \%$ polyacrylamide gels, together with prestained molecular weight standards (BioRad) and separated using a discontinuous buffer system (Laemmli, 1970). Samples from all experimental conditions were always run on the same gel. Gels were run in a MiniProtean III apparatus (Bio-Rad) at a constant voltage $(180 \mathrm{~V})$ for $45 \mathrm{~min}$ at RT. The resolved proteins were transferred onto PVDF membranes at $70 \mathrm{~V}$ for $90 \mathrm{~min}$ at $4^{\circ} \mathrm{C}$.

Blots were blocked for 10-30 min in Tris-buffered saline containing $0.05 \%$ Tween-20 (TBST) with $2.5-5 \%$ milk to minimize non-specific binding. The blots were then probed overnight with primary antibodies for p-PERK (Thr 981) (Santa Cruz), peIF2 $\alpha$ (Ser51) (Cell Signaling), ATF4 (Santa Cruz), ATF3 (Santa Cruz), GADD34 (Santa Cruz), GRP78 (StressMarq), or GRP94 (StressGen). Please see Appendix E for information regarding a number of steps taken in this thesis to ensure antibody specificity. Antibodies were diluted 1:250-1:1000. After incubation, blots were washed several times with TBST and then incubated at RT for $1.5 \mathrm{~h}$ with HRP-linked goat antirabbit IgG (Cell Signaling, diluted 1:2000 in TBST). Immunoblots were developed using enhanced chemiluminescence (ECL) reagents.

\subsubsection{Preparation of nuclear extracts}

Nuclear extracts were prepared using a slight modification of the method described by Dignam et al. (1983). Briefly, $0.5 \mathrm{~g}$ samples were homogenized using a Dounce homogenizer in $1 \mathrm{~mL}$ of homogenization buffer $(10 \mathrm{mM}$ Hepes, $10 \mathrm{mM} \mathrm{KCl}$, $10 \mathrm{mM}$ EDTA, $1 \mathrm{mM}$ DTT, pH 7.9). The DTT, as well as $10 \mu \mathrm{L}$ of Protease Inhibitor Cocktail (Sigma) were added just prior to homogenization. Samples were centrifuged at $10,000 \times g$ for $10 \mathrm{~min}$ at $4^{\circ} \mathrm{C}$ and the supernatant (cytoplasmic extract) was removed. The 
pellet was resuspended in $150 \mu \mathrm{L}$ of extraction buffer (20 mM HEPES, pH 7.9; $400 \mathrm{mM}$ $\mathrm{NaCl} ; 1 \mathrm{mM}$ EDTA; $10 \% \mathrm{v} / \mathrm{v}$ glycerol; $1 \mathrm{mM}$ DTT). The DTT, as well as $1.5 \mu \mathrm{L}$ of Protease Inhibitor Cocktail (Sigma) were added just prior to addition of the buffer to the pellet. Tubes containing the samples were put on ice horizontally on a rocking platform for $1 \mathrm{~h}$. Samples were then centrifuged at $10,000 \times g$ for $10 \mathrm{~min}$ at $4{ }^{\circ} \mathrm{C}$. The supernatant (nuclear extract) was collected. The extracts were quantified and treated as described above to create samples for western immunoblotting. The integrity of the nuclei was confirmed by immunoblotting of cytoplasmic and nuclear fractions and probing with a histone $\mathrm{H} 3$ antibody specific for animals ranging from drosophila to mammals (Cell Signaling; diluted 1:1000 in TBST).

\subsubsection{DNA-binding assay}

Nuclear extracts were prepared as above, with the exception that following quantification, the soluble protein concentrations of the extracts were adjusted to $8 \mu \mathrm{g} / \mu \mathrm{L}$ with extraction buffer without further manipulation. Aliquots containing equal amounts of protein from each sample (10-16 $\mu \mathrm{g} / \mathrm{well}$, depending on tissue) were then used to assess the amount of binding by ATF4 to its response element. This was done using an ELISA-type assay that uses 96-well microplates that were previously coated with an oligonucleotide corresponding to the DNA binding element that is recognized by ATF4.

The sequence of the biotin-conjugated probe was 5'-BiotinCTCCCATTTCCATGACGTCATGGTTA-3', whereas the complementary sequence was 5'-TAACCATGACGTCATGGAAATGGGAG-3'. Both oligonucleotides were 
purchased from Sigma Genosys, diluted to $500 \mu \mathrm{M}$ using sterile $\mathrm{H}_{2} \mathrm{O}$ and subsequently mixed 1:1 (v/v) for a total volume of $20 \mu \mathrm{l}$. Probes were then placed in a thermocycler for $10 \mathrm{~min}$ at $95^{\circ} \mathrm{C}$ and the temperature of the block was slowly cooled to room temperature. A $40 \mathrm{pmol}$ aliquot of double-stranded, biotinylated probe in $50 \mu \mathrm{L}$ of phosphate buffered saline (PBS; $137 \mathrm{mM} \mathrm{NaCl}, 2.7 \mathrm{mM} \mathrm{KCl}, 10 \mathrm{mM} \mathrm{Na} 2 \mathrm{HPO}_{4}, 2 \mathrm{mM} \mathrm{KH}_{2} \mathrm{PO}_{4}, \mathrm{pH} 7.4$ ) was then added to each well of a streptavidin-coated microplate (VWR). The plate was then incubated at RT for $1 \mathrm{~h}$ and washed twice with wash buffer $(0.1 \%$ Tween-20 in PBS) and once with PBS. Aliquots of $10-16 \mu \mathrm{g}$ protein were combined with $50 \mu \mathrm{L}$ of $1 \mathrm{x}$ protein binding buffer [10 mM Hepes, pH 7.9, $50 \mathrm{mM} \mathrm{KCl,} 0.5 \mathrm{mM}$ EDTA, $3 \mathrm{mM}$ $\mathrm{MgCl}_{2}, 10 \%$ glycerol, $0.5 \mathrm{mg} / \mathrm{mL}$ BSA, $0.05 \%$ NP-40, $1-2 \mu \mathrm{g}$ salmon sperm DNA (BioShop), $0.5 \mathrm{mM} \mathrm{DTT}$, and $\mathrm{NaCl}$ (a variable amount was used based on the volume of nuclear extract added, final concentration was always $40 \mathrm{mM}$ )] and then added to the wells. Equal amounts of protein for all experimental conditions were used in each assay, and all experimental samples were always run together. Only binding buffer, without protein samples, was added to the negative control wells. The plate was incubated at room temperature with mild agitation for 75 min followed by 4 washes with wash buffer. A $60 \mu \mathrm{L}$ aliquot of the ATF4 antibody (the same one used for western blotting), diluted 1:1000 in PBS was added to the wells and the plate was incubated for $1 \mathrm{~h}$. The plate was washed as above and then $60 \mu \mathrm{L}$ of anti-rabbit IgG-HRP (BioShop) diluted 1:2000 in PBS was added and the plate was incubated for $1 \mathrm{~h}$. Following this, the plate was washed as above and $60 \mu \mathrm{L}$ of TMB (tetramethylbenzidine) was added (Bioshop). Once colour had developed, the reaction was stopped by the addition of $1 \mathrm{M} \mathrm{HCl}$ and optical density was read at $450 \mathrm{~nm}$ with a reference wavelength of $655 \mathrm{~nm}$ using a Multiskan Spectrum 
(Thermo Labsystems).

\subsubsection{Data and statistics}

Bands on agarose or SDS-PAGE gels were scanned using a ChemiGenius BioImaging system and densitometric analysis was performed using the associated GeneTools software (Syngene, Frederick, MD). RT-PCR bands for all genes were normalized against bands for $\alpha$-tubulin amplified from the same cDNA sample. $\alpha$-tubulin was also checked against $\beta$-actin to ensure it remains constant throughout the anoxic episodes. To control for loading irregularities in western blots, the band intensity of immunoreactive material in each lane was normalized against the combined density of a group of Coomassie stained protein bands on the blot that were constant between control and experimental conditions and that were not located close to the protein band of interest, as previously described (Krivoruchko and Storey, 2010a-d). These have been tested in the past against several other constant proteins in these tissue preparations and stresses. Examples of constantly expressed proteins are listed in Appendix F. To normalize ELISA results, the OD from the negative control was subtracted from the OD of each sample. Mean normalized band densities \pm SEM were then calculated for control and anoxic samples. Data were analyzed using ANOVA and significant differences between control and anoxic values were identified using the Dunnett's post-hoc test. 


\subsection{RESULTS}

\subsubsection{Phosphorylation of PERK and eIF2 $\alpha$}

The relative phosphorylation state of selected key residues on a protein can often be determined using an antibody that recognizes the specific short peptide sequence containing the phosphorylated amino acid residue. Changes in the phosphorylated forms of the kinase PERK and the $\alpha$ subunit of eIF2 in response to anoxia were assessed using immunoblotting (FIG. 2.2). The polyclonal anti-p-PERK (Thr 981) antibody crossreacted with a protein band of $\sim 140 \mathrm{kDa}$, the expected size of PERK. All tissues examined showed an increase in the phosphorylation state of PERK in response to anoxia (FIG. 2.2A). Levels of p-PERK in both heart and white skeletal muscle remained stable after $5 \mathrm{~h}$ of anoxia exposure, but increased to $1.4 \pm 0.1$ and $2.2 \pm 0.1$ fold higher than controls (both $\mathrm{P}<0.05$ ) in response to $20 \mathrm{~h}$ of anoxia, respectively. Levels of p-PERK in kidney increased by $1.4 \pm 0.1$ fold in response to $5 \mathrm{~h}$ of anoxia $(\mathrm{P}<0.05)$ and to $2.0 \pm$ 0.05 fold higher in response to $20 \mathrm{~h}$ of anoxia $(\mathrm{P}<0.01)$. Similarly, liver showed an increase of $1.6 \pm 0.1$ fold in response to $5 \mathrm{~h}$ of anoxia and a rise to $2.0 \pm 0.02$ fold greater than controls in response to $20 \mathrm{~h}$ of anoxia (both $\mathrm{P}<0.01$ ).

The polyclonal anti-p-eIF $2 \alpha$ antibody cross-reacted with a protein band of $\sim 40$ $\mathrm{kDa}$, the expected size of eIF2 $\alpha$. FIG.2.2B shows the changes in the relative protein levels of p-eIF $2 \alpha(\operatorname{Ser} 51)$ in response to anoxia. In both heart and kidney, levels of $\mathrm{p}$ eIF $2 \alpha$ remained constant in response to $5 \mathrm{~h}$ of anoxia, but increased to $2.5 \pm 0.4$ and $1.4 \pm$ 0.1 fold higher than controls in response to $20 \mathrm{~h}$ of anoxia (both $\mathrm{P}<0.05$ ), respectively. In the liver, p-eIF $2 \alpha$ levels rose by $1.8 \pm 0.1$ in response to $5 \mathrm{~h}$ of anoxia and remained at 
$1.8 \pm 0.3$ at $20 \mathrm{~h}$ (both $\mathrm{P}<0.05$ ). No changes in the phosphorylation status of eIF2 $\alpha$ were observed in the muscle.

\subsection{2. cDNA cloning of full-length turtle atf4}

Using RT-PCR and primers derived from the consensus sequence of atf4 from other vertebrates, a PCR product of 821 bp was retrieved from the total RNA prepared from turtle liver. The product was confirmed as encoding a portion of the atf 4 sequence and then 5' and 3' RACE were used to extend the sequence and a final assembled sequence of 1441 bp was obtained. FIG. 2.3 shows the nucleotide sequence of atf 4 and the three possible open reading frames (ORFs). The atf4 transcript is translated in an unusual manner and its translation is largely dependent on the presence of upstream reading frames (uORFs) in the 5' of its mRNA (described further in the Discussion) (Wek and Cavener, 2007). The turtle sequence contained 2 upstream open reading frames (uORFs) in its 5' region, as previously documented in other species; Fig. 2.3 shows ORF1 in bold and boxed, ORF2 in bold and underlined, and the third ORF coding for ATF4 is shaded. FIG. 2.4 shows the translated amino acid sequence of turtle ATF4 aligned with sequences from human (Homo sapiens), chicken (Gallus gallus), western clawed frog (Xenopus tropicalis) and zebrafish (Danio rerio) whereas FIG. 2.5 shows a homology tree (FIG. 2.5A) and a homology matrix (FIG. 2.5B) showing the percent identities between the protein sequences of T.s. elegans ATF4 and the other vertebrates. The turtle atf4 encoded a 349 amino acid protein, compared to 336-354 aa for the other vertebrates. Turtle ATF4 protein was $81.6 \%$ identical with the chicken protein and $70.1 \%$ 
identical with the human ATF4. The turtle sequence did not appear to have any turtlespecific amino acid substitutions.

\subsubsection{Transcript and protein expression of ATF4 in turtle tissues}

Changes in the relative transcript levels of atf 4 in response to 5 and $20 \mathrm{~h}$ of anoxia were assessed using RT-PCR. FIG. 2.6A shows both representative RT-PCR bands of atf 4 amplicons with corresponding $\alpha$-tubulin bands, as well as a graphical representation of the changes in the relative levels of these transcripts in response to anoxia. While atf4 transcript levels remained constant in the heart and muscle throughout the anoxic episode, both kidney and liver displayed significant increases in atf 4 levels, to $1.5 \pm 0.15$ $(\mathrm{P}<0.05)$ and $1.8 \pm 0.1$ fold $(\mathrm{P}<0.01)$ higher than control levels, respectively. Transcript levels returned to normoxic values by $20 \mathrm{~h}$ in both tissues.

Changes in ATF4 protein levels in response to anoxia were assessed via immunoblotting. The polyclonal anti-ATF4 antibody cross-reacted with a protein band of $\sim 40 \mathrm{kDa}$, the expected size of the ATF4 protein. FIG. 2.6B shows the changes in the relative protein levels of ATF4 in response to anoxia. In the heart, ATF4 levels were unchanged in response to $5 \mathrm{~h}$ of anoxia, but rose in response to $20 \mathrm{~h}$ of anoxia by $1.9 \pm$ 0.3 fold $(\mathrm{P}<0.05)$. In the kidney, levels of ATF4 increased by $2.4 \pm 0.1$ fold in response to $5 \mathrm{~h}$ of anoxia $(\mathrm{P}<0.05)$ and remained elevated at $2.2 \pm 0.4$ fold above controls after 20 $\mathrm{h}$ of anoxia $(\mathrm{P}<0.05)$. Liver ATF4 levels increased by $1.6 \pm 0.1$ fold in response to $5 \mathrm{~h}$ of anoxia $(\mathrm{P}<0.01)$ and returned to normoxic levels by $20 \mathrm{~h}$ of anoxia. Muscle was the only tissue to show a decrease in the levels of ATF4 protein during anoxia; levels decreased to 
$48 \pm 6 \%$ and $58 \pm 7 \%$ of control values in response to 5 and $20 \mathrm{~h}$ of anoxia, respectively (both $\mathrm{P}<0.05$ ).

\subsubsection{Nuclear expression and DNA-binding activity of ATF4 in turtle tissues}

In order to assess whether increased ATF4 expression corresponded with increased translocation of this transcription factor into the nucleus, western immunoblotting was performed on nuclear extracts from turtle tissues (FIG. 2.7A). In the nuclear fraction from heart, ATF4 levels increased by $1.4 \pm 0.16$ fold in response to $5 \mathrm{~h}$ of anoxia $(\mathrm{P}<0.05)$ and rose to $2.1 \pm 0.1$ fold greater than controls after $20 \mathrm{~h}$ of anoxia $(\mathrm{P}<$ $0.01)$. Similarly, in the kidney nuclear fraction ATF4 increased by $1.6 \pm 0.1$ fold in response to $5 \mathrm{~h}$ of anoxia and remained elevated $(1.7 \pm 0.2$ fold $)$ after $20 \mathrm{~h}$ of anoxia (both $\mathrm{P}<0.05$ ). Liver displayed a $1.4 \pm 0.04$ fold increase in the nuclear fraction in response to $5 \mathrm{~h}$ of anoxia $(\mathrm{P}<0.05)$, with levels returning to normoxic values by $20 \mathrm{~h}$.

Changes in the DNA-binding activity of nuclear ATF4 in response to anoxia were assessed using a transcription factor binding assay and are presented in FIG. 2.7B. Such changes can be due to changed amounts of ATF4 in the nucleus, a change in the DNAbinding ability of the protein, or both. In the heart, the DNA-binding activity of ATF4 was increased by $1.7 \pm 0.1$ fold in response to $5 \mathrm{~h}$ of anoxia $(\mathrm{P}<0.05)$ and by $2.2 \pm 0.2$ fold in response to $20 \mathrm{~h}$ of anoxia $(\mathrm{P}<0.01)$, compared with controls. Similarly, kidney ATF4 showed $1.5 \pm 0.1$ and $1.9 \pm 0.15$ fold increases in binding activity in response to 5 or $20 \mathrm{~h}$ of anoxia, respectively (both $\mathrm{P}<0.01$ ). In nuclear extracts from liver, ATF4 DNA-binding activity increased by $2.3 \pm 0.45$ fold in response to $5 \mathrm{~h}$ of anoxia $(\mathrm{P}<0.05)$ 
but returned to normoxic values after $20 \mathrm{~h}$ of anoxia.

\subsection{5. cDNA cloning of partial turtle atf3}

A PCR product of $333 \mathrm{bp}$ was retrieved from the total RNA prepared from turtle liver. The product was confirmed as encoding a portion of the atf3 sequence by BLAST. FIG. 2.8A shows the partial nucleotide sequence with the corresponding amino acid sequence. The corresponding amino acid sequence was 111 amino acids in length, corresponding to amino acids $15-125$ of the chicken ATF3 sequence. This sequence represents $61 \%$ of the total ATF3 sequence. FIG. 2.8B shows a homology tree produced from the alignment of the partial T. s. elegans ATF3 protein sequence with ATF3 from human, chicken, African clawed frog (Xenopus laevis) and zebrafish. The chicken ATF3 sequence was the closest to the turtle sequence, showing an $88 \%$ similarity.

\subsubsection{Expression of ATF3 in response to anoxia}

Relative transcript levels of atf3, a target gene of ATF4, were assessed using RTPCR. FIG. 2.9A shows both representative RT-PCR bands of atf 3 amplicons with corresponding a $\alpha$-tubulin bands, as well as a graphical representation of the changes in the relative levels of these transcripts in response to anoxia. Heart atf 3 levels remained constant in response to $5 \mathrm{~h}$ of anoxia, but rose to $2.1 \pm 0.1$-fold of control values $(\mathrm{P}<$ 0.01 ) in response to $20 \mathrm{~h}$ of anoxia. In the kidney, atf levels also remained stable in response to $5 \mathrm{~h}$ of anoxia, but decreased to $54 \pm 7 \%$ of the control by $20 \mathrm{~h}(\mathrm{P}<0.05)$. In 
the liver, at 3 levels were also unchanged after $5 \mathrm{~h}$ of anoxia, but strongly decreased to 14 $\pm 1 \%$ of control values by $20 \mathrm{~h}$ of anoxia $(\mathrm{P}<0.01)$.

The relative protein levels of ATF3 were examined using western blotting. Using the mammalian ATF3 antibody, a band of $\sim 20 \mathrm{kDa}$ was detected, corresponding to the known molecular weight of ATF3. FIG. 2.9B shows that expression of ATF3 protein remained stable in the heart in response to $5 \mathrm{~h}$ of anoxia, but increased to $1.6 \pm 0.1$ fold of control in response to $20 \mathrm{~h}(\mathrm{P}<0.05)$. Kidney ATF3 also remained stable after $5 \mathrm{~h}$ anoxia, but decreased to $54 \pm 10 \%$ of the control after $20 \mathrm{~h}$ of anoxia $(\mathrm{P}<0.01)$. In liver, ATF3 increased to $3.3 \pm 0.25$ fold of control in response to $5 \mathrm{~h}$ of anoxia $(\mathrm{P}<0.01)$, returning to normoxic values by $20 \mathrm{~h}$.

\subsubsection{Expression of GADD34 in response to anoxia}

Changes in the relative protein expression of GADD34, a downstream target of ATF4, in response to anoxia were assessed using western blotting. The polyclonal GADD34 antibody cross-reacted with a band of $\sim 75 \mathrm{kDa}$, corresponding to the known molecular weight of GADD34. FIG. 2.10 shows that GADD34 expression remained stable in the heart in response to $5 \mathrm{~h}$ of anoxia, but increased to $1.4 \pm 0.05$ fold higher than control $(\mathrm{P}<0.05)$ in response to $20 \mathrm{~h}$ of anoxia. In the liver, GADD34 protein levels increased by $1.8 \pm 0.1$ fold in response to $5 \mathrm{~h}$ of anoxia $(\mathrm{P}<0.05)$ and returned to normoxic levels by $20 \mathrm{~h}$ of anoxia. No significant changes in GADD34 expression were observed in the kidney in response to anoxia. 


\subsection{8. cDNA cloning of partial turtle grp78}

A PCR product of $837 \mathrm{bp}$ was retrieved using the original $g r p 78$ primers from the total RNA prepared from turtle liver. The product was confirmed as encoding a portion of the $\operatorname{grp} 78$ sequence by BLAST. FIG. 2.11A shows the partial nucleotide sequence with the corresponding 279 amino acid sequence that corresponded to amino acid residues 87365 of the chicken GRP78 sequence. This sequence represents $43 \%$ of the full chicken GRP78 sequence. FIG. 2.11B shows a homology tree produced from the alignment of the partial T. s. elegans GRP78 protein sequence with GRP78 from human, chicken, African clawed frog and zebrafish. The chicken GRP78 sequence was the closest to the turtle sequence, showing $99 \%$ similarity.

\subsubsection{Expression of GRP78 in response to anoxia}

Relative transcript levels of $g r p 78$ were assessed using RT-PCR. FIG. 2.12A shows both representative RT-PCR bands of $g r p 78$ amplicons with corresponding $\alpha$ tubulin bands, as well as a graphical representation of the changes in the relative levels of these transcripts in response to anoxia. Levels of $g r p 78$ in heart increased by $1.7 \pm 0.2$ fold in response to $5 \mathrm{~h}$ of anoxia $(\mathrm{P}<0.05)$ and returned to normoxic values by $20 \mathrm{~h}$ of anoxia. Similarly, kidney showed a $1.8 \pm 0.2$ fold $(\mathrm{P}<0.05)$ rise in grp 78 levels in response to $5 \mathrm{~h}$ of anoxia, whereas in liver transcript levels rose by $2.2 \pm 0.1$ fold $(\mathrm{P}<$ $0.01)$. Transcript levels in both tissues returned to nomoxic levels by $20 \mathrm{~h}$ of anoxia.

The relative protein levels of GRP78 were examined using western blotting. Using the mammalian GRP78 antibody, a band of $\sim 78 \mathrm{kDa}$ was detected, corresponding 
to the known molecular weight of GRP78. FIG. 2.12B shows representative western blots of GRP78 in turtle tissues, as well as a histogram showing the changes in GRP78 levels in response to anoxia. In both heart and kidney, levels of GRP78 remained stable in response to $5 \mathrm{~h}$ of anoxia, but increased to $1.5 \pm 0.1$ and $1.4 \pm 0.02$ fold higher than controls in response to $20 \mathrm{~h}$ of anoxia, respectively (both $\mathrm{P}<0.01$ ). Liver showed a $2.2 \pm$ 0.2 fold increase in response to $5 \mathrm{~h}$ of anoxia, with levels remaining elevated at $1.8 \pm 0.1$ fold at $20 \mathrm{~h}$ of anoxia (both $\mathrm{P}<0.05$ ).

\subsubsection{0. cDNA cloning of partial turtle grp94}

A PCR product of $955 \mathrm{bp}$ was retrieved using the original grp 94 primers from the total RNA prepared from turtle liver. The product was confirmed as encoding a portion of the grp94 sequence by BLAST. FIG. 2.13A shows the partial nucleotide sequence with the translated amino acid sequence of 317 residues that corresponded to amino acids 329 623 of chicken GRP94. This represents $40 \%$ of the chicken sequence. FIG. 2.13B shows a homology tree produced from the alignment of the partial $T$. s. elegans GRP94 protein with GRP94 sequences from human, chicken, African clawed frog and zebrafish. Chicken GRP94 was the closest to the turtle sequence, showing a $92 \%$ similarity.

\subsubsection{Expression of GRP94 in response to anoxia}

Relative transcript levels of $\operatorname{grp} 94$, a target gene of ATF4, were assessed using RT-PCR. FIG. 2.14A shows both representative RT-PCR bands of grp 94 amplicons with 
corresponding $\alpha$-tubulin bands, as well as a graphical representation of the changes in the relative levels of these transcripts in response to anoxia. In heart, grp94 transcript levels increased by $2.9 \pm 0.3$ fold compared with control in response to $5 \mathrm{~h}$ of anoxia $(\mathrm{P}<0.01)$ and remained elevated at $2.1 \pm 0.3$ fold higher at $20 \mathrm{~h}$ of anoxia $(\mathrm{P}<0.05)$. In liver, a strong $4.5 \pm 0.4$ fold increase was observed in response to $5 \mathrm{~h}$ of anoxia $(\mathrm{P}<0.01)$, but grp 94 transcripts returned to normoxic values by $20 \mathrm{~h}$ of anoxia. No change in grp 94 transcript levels occurred in kidney in response to anoxia.

The relative protein levels of GRP94 were measured using western blotting. A band of $\sim 94 \mathrm{kDa}$ was detected, corresponding to the known molecular weight of GRP94. FIG. 2.14B shows representative western blots of GRP94 in turtle tissues and a histogram showing mean GRP94 levels in response to anoxia. In heart, GRP94 levels remained stable after $5 \mathrm{~h}$ of anoxia but increased by $3.2 \pm 0.6$ fold after $20 \mathrm{~h}$ of anoxia (P $<0.01$ ). Liver displayed a $2.4 \pm 0.1$ fold increase in GRP94 protein levels in response to 5 $\mathrm{h}$ of anoxia $(\mathrm{P}<0.05)$, with GRP94 levels returning to normoxic levels by $20 \mathrm{~h}$. No change in GRP94 levels were seen in kidney in response to anoxia.

\subsection{DISCUSSION}

A large body of research has shown that perturbations in any of the main homeostatic functions of the endoplasmic reticulum (ER) can lead to the accumulation of unfolded proteins in the ER lumen, triggering the unfolded protein response (UPR). This response subsequently functions to alleviate ER stress by (1) reducing the folding demand on the ER through inhibition of protein synthesis (2) increasing the folding 
capacity of the ER by inducing the synthesis of chaperones and (3) degrading slowly folding or permanently unfolded proteins (Schroder and Kaufman, 2005a,b). Besides the metabolic stress of oxygen deprivation, anoxia in turtles is also characterized by decreased intracellular pH (Jackson and Heisler 1983; Wasser et al. 1991) as well as an altered redox environment (Herbert and Jackson, 1985). These conditions could potentially interfere with the function of the ER and lead to the accumulation of unfolded proteins, triggering the unfolded protein response. Previous studies have shown that activation of stress-responsive proteins and transcription factors occurs in response to anoxia in turtle tissues. For example, previous studies in our lab have shown that the heat shock response is induced in turtle tissues in response to anoxia (Krivoruchko and Storey, 2010b). This response involves the proliferation of many molecular chaperones and its activation in turtle tissues highlights the importance of stress-responsive pathways to long-term anoxic survival. Activation of the UPR in response to anoxia could have similar protective effects during anoxic submergence, and studies with mammalian cells have already implicated components of the UPR in hypoxia resistance, showing that cells with compromised PERK- eIF2 $\alpha$-ATF4 signaling are more impaired by hypoxia (Fels and Koumenis, 2006). In addition, components of the UPR have been shown to be induced in mammalian hibernation (Mamamdy and Storey, 2008), another state characterized by profound metabolic rate depression. Because of its potential importance to turtle anoxia tolerance, I hypothesized that this response would be activated in turtle tissues in response to anoxia. The present chapter shows that various UPR-related proteins are induced in response to anoxia in turtle tissues, suggesting that the UPR is an important mechanism of cellular protection during this stress. 
PERK and eIF $2 \alpha$ are phosphorylated in turtle tissues in response to anoxia

Double-stranded RNA-activated protein kinase-like ER kinase (PERK) is one of the three main stress sensors in the ER. This protein is a type I transmembrane protein kinase with both luminal and cytoplasmic domains. Under basal conditions it is found in an inactive state in association with GRP78 (BiP), but ER stress and the subsequent accumulation of unfolded proteins drives GRP78 to favour association with those unfolded proteins instead, dissociating from PERK and allowing PERK to become activated through phosphorylation and oligomerization (Ron and Walter, 2007). Therefore, an increase in the levels of phosphorylated PERK can be used as a marker for the activation of the UPR. The present data show that elevated amounts of the phosphorylated form of PERK occur in all tissues examined in response to anoxia exposure, with levels rising by 1.4-2.2 fold (FIG.2.2A). Interestingly, a difference in the time-dependent activation of PERK was observed between different tissues. Kidney and liver displayed a rapid activation of PERK, with p-PERK levels increasing within $5 \mathrm{~h}$ of anoxic submergence, while both cardiac and skeletal muscles only showed increased levels after $20 \mathrm{~h}$.

The best characterized substrate of PERK is the $\alpha$ subunit of the eukaryotic initiation factor 2 , and phosphorylation of this subunit leads to inhibition of translation, thereby reducing the influx of unfolded proteins into the ER. Because this process does not require time-consuming transcription and translation, it is the most immediate response by the ER to unfolded protein accumulation. There appeared to be some 
correlation between the phosphorylation of PERK and eIF $2 \alpha$ with levels of p-eIF $2 \alpha$ increasing by 1.4-2.5 fold in response to anoxia in most turtle tissues (FIG.2.2B). Interestingly, while skeletal muscle tissue displayed a 2.2-fold upregulation in p-PERK levels in response to anoxia, no increase in p-elF2 $\alpha$ levels was observed. A possible explanation could be that the activated PERK phosphorylates a substrate other than eIF2 $\alpha$ in response to anoxia in this tissue. A potential candidate is the transcription factor Nrf2, another important substrate of PERK (Cullinan et al., 2003). Nrf2 can bind to the antioxidant response element and activate the transcription of various antioxidant genes, thereby serving as an important mediator of the antioxidant defense response of the cell. In unstressed cells, $\mathrm{Nrf2}$ is maintained in the cytoplasm through association with the inhibitor Keap1. PERK-dependent phosphorylation of Nrf2 triggers its dissociation from Keap 1 and frees Nrf2 to activate transcription of antioxidant genes (Cullinan et al., 2003). It has been shown that PERK-dependent activation of Nrf2 contributes to cell survival and redox homeostasis following ER stress in mammalian cells (Cullinan and Diehl, 2004).

Under anoxic conditions, the electron carriers in the respiratory chain become strongly reduced and their immediate reoxidation upon recovery could potentially lead to oxidative stress in the cell. The turtle appears to have both strong constitutive antioxidant defenses (Willmore and Storey, 1997a,b), as well as anoxia-inducible antioxidant defenses (discussed further in Chapters 3 and 4) that allow it to endure multiple bouts of anoxia and reoxygenation without any apparent tissue damage. PERK-mediated activation of Nrf2 in response to anoxia in turtle muscle could potentially be an important mechanism of antioxidant gene activation in this animal. Interestingly, previous studies in 
frozen hatchling turtles (Krivoruchko and Storey, 2010a), as well as in hibernating ground squirrels (Morin et al., 2008), have shown that Nrf2 becomes activated in response to these stresses.

It should be noted that other than being an important component of the ER stress response, translational inhibition is also crucial for metabolic rate depression in turtle cells in response to anoxia. Indeed, previous studies have found that the rate of protein synthesis in turtles decreases in response to anoxia (Fraser et al., 2001; Land et al., 1993), and ribosomal factors such as the eukaryotic elongation factor 2 are inactivated (Rider et al., 2009). It is possible therefore, that activation of the UPR through the PERK- eIF2 $\alpha$ pathway in turtle tissues not only relieves stress, but also contributes to metabolic rate depression in these tissues through translational inhibition.

\section{ATF4 sequence analysis}

In addition to translational inhibition, PERK-mediated UPR activation also leads to induction of the important stress-responsive transcription factor ATF4. ATF4 is known to be induced in response to a variety of stresses, including oxygen deprivation (hypoxia/anoxia), amino acid deprivation, ER stress and oxidative stress (Ameri et al., 2004; Blais et al., 2004; Harding et al., 2003). ATF4 can function as both a transcriptional activator and a repressor and is involved in the induction of genes involved in mitochondrial function, amino acid metabolism and redox chemistry and protein folding (Harding et al., 2003).

The atf4 mRNA transcript has several upstream open reading frames (uORFs) in 
its 5' region. These uORFs play a crucial role in the regulation of ATF4 activation through eIF2 $\alpha$ phosphorylation (Wek and Cavener, 2007). It is proposed that translation of the atf 4 gene is initiated at the uORF1 of atf4. After synthesis of the uORF1-encoded tripeptide, ribosomes continue binding to the atf $4 \mathrm{mRNA}$ and reinitiate translation at the downstream coding region. In unstressed cells, where eIF $2 \alpha$ phosphorylation is low and abundant levels of eIF2-GTP are present, ribosomes scanning downstream from uORF1 rapidly reinitiate translation of the next ORF, uORF2 (Wek and Cavener, 2007). Because uORF2 overlaps with the atf 4 coding region, ATF4 translation is inhibited. However, under stress conditions, elevated phosphorylation of eIF $2 \alpha$ reduces the levels of eIF2-GTP, increasing the time required for the scanning ribosome to become competent to reinitiate translation (Wek and Cavener, 2007). Therefore, after translation of uORF1, reinitiation of translation will be delayed, resulting in a bypass of the uORF2 initiation codon and the start of translation at the initiation codon for ATF4 proper. Therefore, ATF4 should rapidly accumulate in response to conditions that elevate eIF $2 \alpha$ phosphorylation, such as ER stress.

The presence of uORFs in the 5' upstream region of atf4 mRNA has been shown in a variety of species, suggesting that this is an evolutionary conserved mechanism of ATF4 regulation. In the present study, cloning of the full-length atf 4 cDNA from $T . s$. elegans found that 2 uORFs were present in the 5 ' region of turtle atf 4 mRNA, the tripeptide-encoding, positive-acting, uORF1, and the inhibitory uORF2, which overlaps with the coding frame of ATF4 (FIG. 2.3). Based on these findings, turtle ATF4 appears to be regulated in the same way as in other vertebrates. FIG.2.4 shows the amino acid alignment of turtle ATF4 with ATF4 from other vertebrates. The turtle ATF4 protein was 
most closely related to the chicken sequence ( $82 \%$ identity), reflecting the close evolutionary relationship between reptiles and birds (FIG. 2.5). No unique amino acid substitutions were observed in the turtle sequence compared to the other species, suggesting that turtle ATF4 does not possess any special structural features that would enhance stabilization under anoxic conditions.

Multiple modes of ATF4 activation in turtle tissues

ATF4 demonstrated a tissue-specific activation pattern in response to anoxia in $T$. s. elegans. While no increase in transcript levels of atf4 was observed in heart, ATF4 protein did increase by 1.9 fold in response to $20 \mathrm{~h}$ of anoxic submergence (FIG.2.6). This increase correlated with the 2.5 fold increase in p-eIF $2 \alpha$ levels observed, and therefore, is likely a result of UPR-mediated translational inhibition in this tissue. In contrast, the kidney displayed a 1.5 fold increase in atf 4 transcript levels in response to 5 $\mathrm{h}$ of anoxia, as well as a 2.4 increase in ATF4 protein levels. Since no increase in eIF2 $\alpha$ phosphorylation was observed in response to $5 \mathrm{~h}$ of anoxia in kidney, the rise in ATF4 protein is likely due to the elevated transcript levels of ATF4. By $20 \mathrm{~h}$ of anoxia atf4 transcript levels returned to normoxic values, but the protein remained elevated at 2.2 fold higher than controls, whereas p-eIF $2 \alpha$ increase by 1.4 fold. Thus, the high ATF 4 protein levels in kidney at this time point may be due either to enhanced eIF $2 \alpha$ phosphorylation, or to maintenance of ATF4 at a new higher level as a result of enhanced synthesis in the earlier hours of anoxia exposure. The liver showed increases of 1.6-1.8 fold in both transcript and protein levels of ATF4 in response to $5 \mathrm{~h}$ of anoxia. 
Since p-eIF $2 \alpha$ levels were also elevated at this point, the increase in ATF4 protein levels could be due to a combination of gene up-regulation and p-eIF $2 \alpha$ regulation of atf 4 translation. No increase in ATF4 protein or transcript levels was seen in skeletal muscle in response to anoxia, suggesting that this transcription factor is not an important component of anoxia tolerance in muscle (at least at the time-points examined).

To obtain further evidence for ATF4 activation in response to anoxia, the nuclear localization and DNA binding activity of the transcription factor were examined (FIG.2.7). Interestingly, whereas no increase in ATF4 protein was seen in heart until $20 \mathrm{~h}$ of anoxia, a 1.4 fold increase in nuclear content of ATF4 was evident by $5 \mathrm{~h}$ as was a corresponding a 1.7-fold increase in the DNA-binding activity. These results suggest that in addition to elevation of transcript levels and p-eIF $2 \alpha-$ mediated regulation of ATF4 translation, activation of ATF4 also occurs by enhanced nuclear localization and/or DNA-binding activity under anoxic conditions. Both nuclear levels and DNA binding activity further increased to 2-2.2 fold higher than control values in response to $20 \mathrm{~h}$ of anoxia in the heart, correlating with the increased ATF4 protein levels observed at this time. In kidney, a 1.6-1.7 fold increase in the nuclear content of ATF4 occurred in response to anoxia, along with a 1.5-1.9 fold increase in DNA-binding activity, also correlating with increased ATF4 protein content in this tissue. Similarly, enhanced ATF4 protein levels in liver in response to $5 \mathrm{~h}$ of anoxia were accompanied by a 1.4-2.3 fold increase in nuclear ATF4 levels and DNA-binding activity. Overall, these results demonstrate a tissue-specific pattern for ATF4 activation in T. s. elegans in response to anoxia, and suggest that this transcription factor is an important component of anoxic survival in this animal. 
Activation of ATF4-depdendent UPR target genes

In addition to examining the state of ATF4 activation in response to anoxia in turtle tissues, the expression of some of its target genes was also examined. ATF3 is a transcription factor that lies downstream of ATF4 and is involved in the induction of a program of gene expression responsible for cellular remediation, as well as apoptosis (Harding et al., 2003). Extensive studies have characterized ATF3 as an adaptiveresponse gene (Hai, 2006; Lu et al., 2006) that is induced by a wide variety of signals including those initiated by cytokines, genotoxic agents, or physiological stresses (Hai, 2006). In addition, studies of mammalian tumors have shown ATF3 induction in response to anoxia (Ameri et al., 2007). In the present study, the anoxia-responsive expression of ATF3 was analyzed by examining both transcript and protein levels of ATF3 (FIG. 2.9). Like the ATF4 sequence, the deduced partial amino acid sequence of ATF3 was mostclosely related to the chicken sequence, showing an $88 \%$ similarity (FIG.2.8).

In the heart, both transcript and protein levels of ATF3 were induced by 1.6-2.1 fold in response to $20 \mathrm{~h}$ of anoxia, likely due to ATF4-mediated up-regulation of atf3 expression. The other tissues, however, displayed different results. No increases in ATF3 transcript or protein levels were observed in response to $5 \mathrm{~h}$ of anoxia in the kidney, suggesting that in this organ ATF3 is not activated in an ATF4-dependent manner in response to anoxia. Moreover, both transcript and protein levels decreased to $54 \%$ of control values after $20 \mathrm{~h}$ of anoxia, a decrease likely due to anoxia-responsive decreases in global transcription and translation rates. The response in the liver was different still. Despite the elevated ATF4 activity observed in response to $5 \mathrm{~h}$ of anoxia, no change in ATF3 transcript levels was observed, and a very strong decrease in atf3 transcripts to just 
$14 \%$ of control values was seen after $20 \mathrm{~h}$ anoxia. Despite this, a significant 3.3 fold increase in ATF3 protein was observed in response to $5 \mathrm{~h}$ of anoxia in the liver, suggesting that this protein is induced by mechanisms other than ATF4-mediated transcriptional activation in this tissue. One such mechanism could be enhanced protein stabilization, which could lead to accumulation of the ATF3 protein in response to anoxia. Indeed, studies in mammalian cells have suggested that protein stabilization contributes to enhanced levels of ATF3 (Yang et al., 2009). If such a mechanism was to stabilize the ATF3 protein in response to anoxia, it could also explain the observation that ATF3 protein levels in liver returned to normoxic values after $20 \mathrm{~h}$ of anoxia, despite the very strong suppression of transcript levels.

Another important ATF4 target is GADD34, which is known to be activated late in the UPR (Novoa et al., 2001). GADD34 is required for feedback control of the UPR by targeting the type 1 serine/threonine protein phosphatase to eIF $2 \alpha$ and dephosphorylating it (Jiang et al., 2004; Connor et al., 2001; Brush et al., 2002). Changes in GADD34 protein levels were measured to determine whether this protein is upregulated in response to anoxia in the turtle (FIG. 2.10). Both heart and liver displayed elevated levels of GADD34, heart GADD34 rising by 1.4 fold after $20 \mathrm{~h}$ of anoxia and liver GADD34 increasing by 1.8 fold after $5 \mathrm{~h}$ of anoxia. ATF 4 was activated in both of these tissues in response to anoxia, suggesting that GADD34 increases in an ATF4depdendent manner in response to anoxia in these tissues. The increase in the heart suggests that the UPR might be subject to inhibition in this tissue at a future time if anoxia was prolonged. Oddly, whereas GADD34 in liver increased in response to $5 \mathrm{~h}$ anoxia, no change in the levels of p-eIF $2 \alpha$ was evident. This observation might suggest 
that GADD34 plays a role other than negative feedback of the UPR in this tissue. For example, some studies have shown increased expression of GADD34 to be associated with reduced cell proliferation and increased expression of the cell cycle inhibitor p21 (Minami et al., 2007; Yagi et al., 2003), which could contribute to the global metabolic rate depression that characterizes anoxia. No change in GADD34 levels was observed in the kidney, suggesting that no inhibition of the UPR was present within the timeframe analyzed.

Activation of UPR-inducible molecular chaperones in response to anoxia

One of the most important functions of the UPR is the up-regulation of molecular chaperones to enhance the folding capacity of the ER. The anoxia-responsive expression of two such chaperones was examined in the present study: the glucose-regulated proteins GRP78 and GRP94. These proteins are ER-resident molecular chaperones that are induced by multiple branches of the UPR in response to anoxia, making them a useful marker for UPR onset (Lee , 2001). GRP78 is known to be induced by all three branches of the UPR, whereas GRP94 is predominantly induced by ATF6 and IRE1 branches. Both of these proteins function in refolding protein substrates, and by doing so relieve ER stress and allow a return to homeostasis (Lee, 2001).

Based on the alignment of the partial T. s. elegans GRP78 amino acid sequence with other vertebrates, this protein appeared to be very highly conserved, having a $99 \%$ similarity with chicken GRP78 and a 93-97\% similarly with other vertebrates (FIG. 2.11). The partial GRP94 sequence was also well-conserved, showing a $92 \%$ similarity 
with the chicken sequence (FIG. 2.13).

Both transcript and protein levels of these chaperones were assessed in $T . s$. elegans tissues in response to anoxia (FIG. 2.12 and 2.14). The heart displayed 1.7-2.9 fold upregulation of $\operatorname{grp} 78$ and $g r p 94$ transcript levels in response to $5 \mathrm{~h}$ of anoxia and levels of grp 94 transcripts remained elevated at 2.1 fold greater than controls after $20 \mathrm{~h}$ anoxia. Protein levels of both also rose by 1.5-3.2 fold in response to $20 \mathrm{~h}$ of anoxia in heart. These results provide a further demonstration of the activation of the UPR in the anoxic heart. In addition, since significant increases in grp transcript levels were observed early in anoxia, it is possible that the other components of the UPR become active early during anoxia in this tissue. Kidney displayed an increase of 1.8 fold in grp 78 transcript levels in response to $5 \mathrm{~h}$ of anoxia, and an increase of 1.4 fold in the protein levels was observed by $20 \mathrm{~h}$ of anoxia, whereas GRP94 transcripts and protein remained stable. Since GRP78 is a downstream target of ATF4, this increase could be mediated by ATF4. If that is the case, a possible reason why GRP94 was not induced could be because GRP94 is not a known target of ATF4. These results also suggest that the other branches of the UPR are not induced at the anoxic time-points examined in the kidney (since GRP94 is usually one of their main targets), although a further examination of these branches is required to support this postulation. In the liver, transcript levels of both proteins increased strongly by 2.2-4.5 fold in response to $5 \mathrm{~h}$ of anoxia, while protein levels increased by 2.2-2.4 fold (and remained elevated at 1.8 fold over controls after $20 \mathrm{~h}$ of anoxia for GRP78). These strong inductions further demonstrate the importance of the UPR in the liver, and like the case in heart, suggest that other branches of the UPR become activated in response to anoxia in this tissue. 


\section{Conclusions}

In summary, the present chapter demonstrates that activation of the UPR occurs in the heart, kidney and liver of $T$. $s$. elegans in response to anoxia. Various components of the UPR including p-PERK, ATF4 and its target genes, and GRPs were elevated in response to anoxia in these tissues, suggesting an important protective role for the UPR in turtle anoxia tolerance. Activation of the UPR early during anoxic submergence could help ensure an appropriate folding state for proteins and the maintenance of protein viability during the transition from an oxygenated state to an anoxic state. While the present study predominantly examined the PERK-eIF2 $\alpha$-ATF4 branch of the UPR, it would be of interest for future studies to examine the activation status of the other UPR branches, controlled by ATF6 and IRE1, in more detail. 
TABLE 2.1. List of primers used in chapter 2.

\begin{tabular}{|c|c|c|c|}
\hline Gene/primer & Primer sequence $\left(5^{\prime}-3^{\prime}\right)$ & $\begin{array}{c}\text { Annealing } \\
\text { temp. } \\
\left({ }^{\circ} \mathrm{C}\right)\end{array}$ & $\begin{array}{c}\text { Expected } \\
\text { product size } \\
\text { (bp) }\end{array}$ \\
\hline atf4 forward & TTCTCCAGCGACCAAGGCTAA & \multirow[b]{2}{*}{62.5} & \multirow[b]{2}{*}{$\sim 900$} \\
\hline atf 4 reverse & CCTTGCGGACCTCTTCTATC & & \\
\hline atf3 forward & GGTGTCTGCATTGGAAGTC & \multirow[b]{2}{*}{54} & \multirow[b]{2}{*}{$\sim 400$} \\
\hline atf3 reverse & CAAATGCTGCTTCTCATTCT & & \\
\hline $\begin{array}{l}\text { grp } 78 \text { original } \\
\text { forward }\end{array}$ & ACCGCATCACGCCGTCTTAT & \multirow{2}{*}{62.5} & \multirow{2}{*}{$\sim 1000$} \\
\hline $\begin{array}{l}\text { grp } 78 \text { original } \\
\text { reverse }\end{array}$ & GCTCCTTGCCATTGAAGAAC & & \\
\hline $\begin{array}{l}\text { grp } 78 \text { perfect } \\
\text { forward }\end{array}$ & TTGATGTTGGTGGTGGACAG & \multirow[t]{2}{*}{62.5} & \multirow[t]{2}{*}{$\sim 600$} \\
\hline $\begin{array}{l}\text { grp } 78 \text { perfect } \\
\text { reverse }\end{array}$ & CTTCATGGTGGAACGGAACA & & \\
\hline $\begin{array}{l}\text { grp } 94 \text { original } \\
\text { forward }\end{array}$ & GTGTGGAGCAGCAAGACAGA & \multirow[t]{2}{*}{62.5} & \multirow[t]{2}{*}{$\sim 1000$} \\
\hline $\begin{array}{l}\text { grp94 original } \\
\text { reverse }\end{array}$ & AGACTGAGTTAAGCGTTGAG & & \\
\hline $\begin{array}{l}\text { grp94 perfect } \\
\text { forward }\end{array}$ & CGATGATGAGGCTGCTGTTG & \multirow[t]{2}{*}{62.5} & \multirow[t]{2}{*}{$\sim 900$} \\
\hline $\begin{array}{l}\text { grp } 94 \text { perfect } \\
\text { reverse }\end{array}$ & CCAGGTTAAGAGTGGCTCAA & & \\
\hline$\alpha$-tubulin forward & GGAAGATGCTGCCAATAACT & \multirow[b]{2}{*}{54} & \multirow[b]{2}{*}{$\sim 500$} \\
\hline$\alpha$-tubulin reverse & GTCTGGAACTCGGTCAGATC & & \\
\hline
\end{tabular}


FIG.2.1

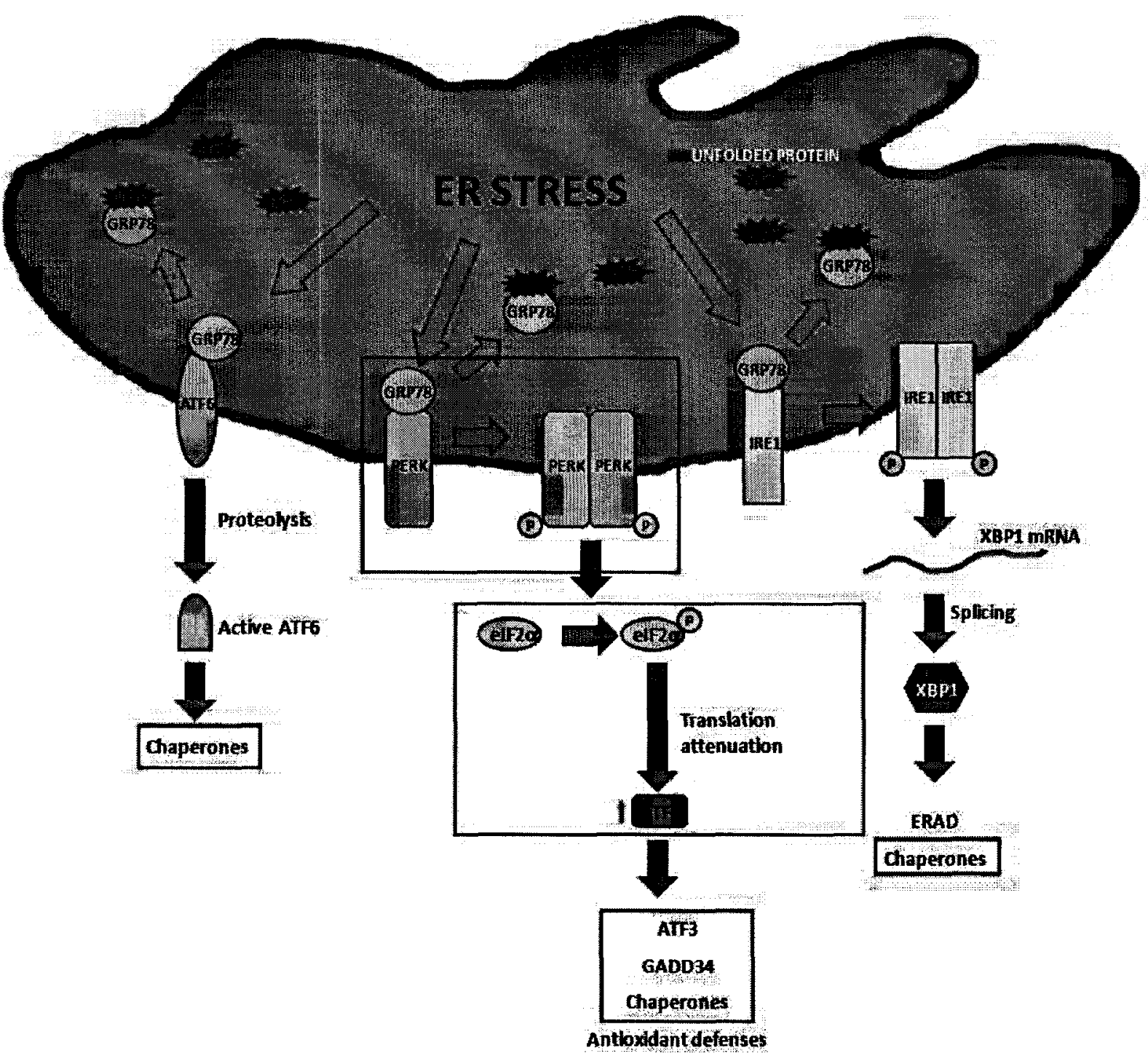

FIG. 2.1. Overview of the Unfolded Protein Response (UPR).

In response to ER stress, the ER-resident stress sensors PERK, IRE1 and ATF6 dissociate from GRP78 and undergo post-translational modifications that activate them.

Phosphorylated PERK phosphorylates the $\alpha$ subunit of the eukaryotic initiation factor 2 (eIF $2 \alpha$ ), thereby inhibiting protein synthesis. Phosphorylation of eIF $2 \alpha$ also triggers the enhanced expression of activation transcription factor 4 (ATF4), which activates the expression of a variety of target genes. The main outcome of the UPR is a reduction in the number of unfolded proteins via inhibition of protein synthesis, refolding of proteins, and ER-associated degradation (ERAD) of permanently unfolded proteins. Note that the parts of the UPR examined in this chapter are boxed. 
FIG. 2.2

A)
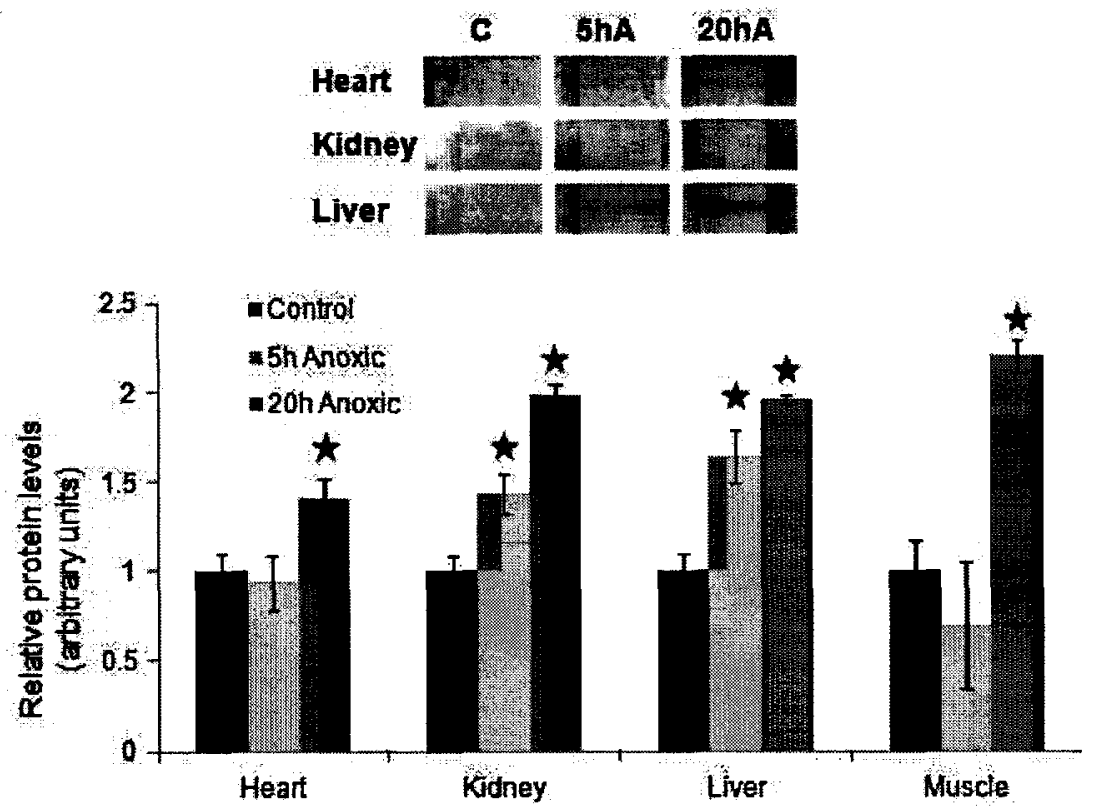

B)

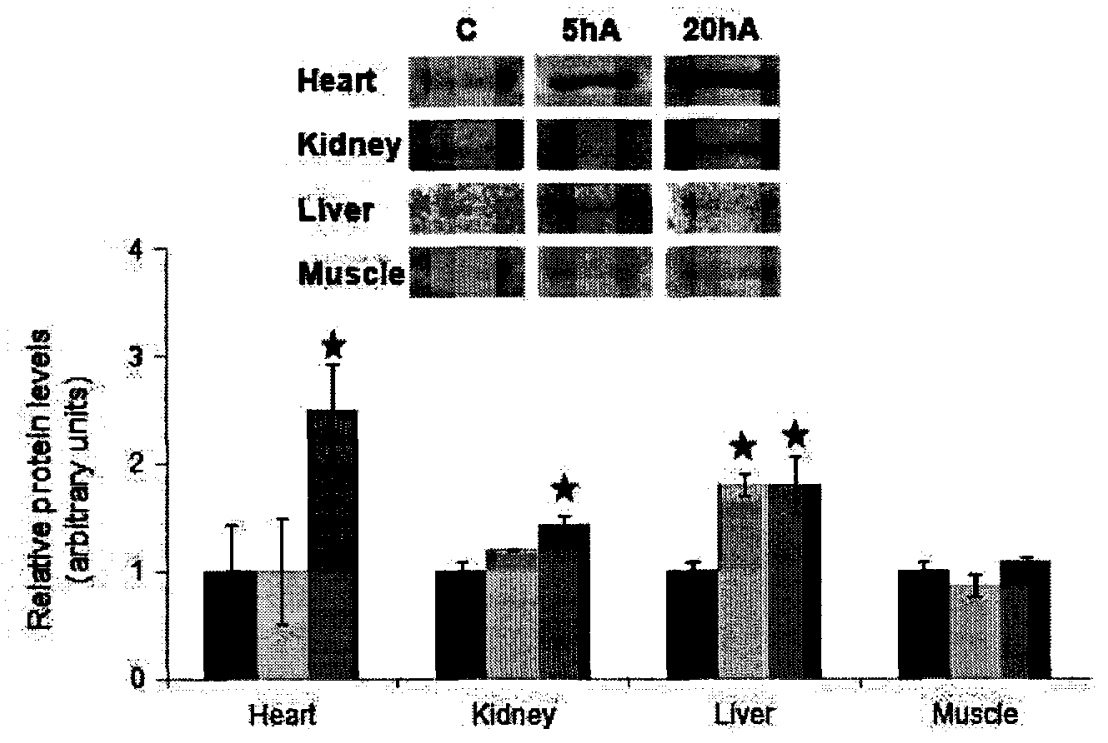

FIG. 2.2. Effect of 5 and $20 \mathrm{~h}$ of anoxic submergence on the phosphorylation status of PERK and eIF2 $\alpha$ in T. s. elegans tissues as determined by Western immunoblotting.

(A) p-PERK (Thr 981) levels, and (B) p-eIF2 $\alpha$ (Ser 51) levels. Representative Western immunoblots show changes in response to anoxia. Histograms show means normalized protein levels in turtle tissues. Data are means \pm SEM, $n=3-5$ independent trials. * Significantly different from the corresponding control, $P<0.05$. 
FIG. 2.3

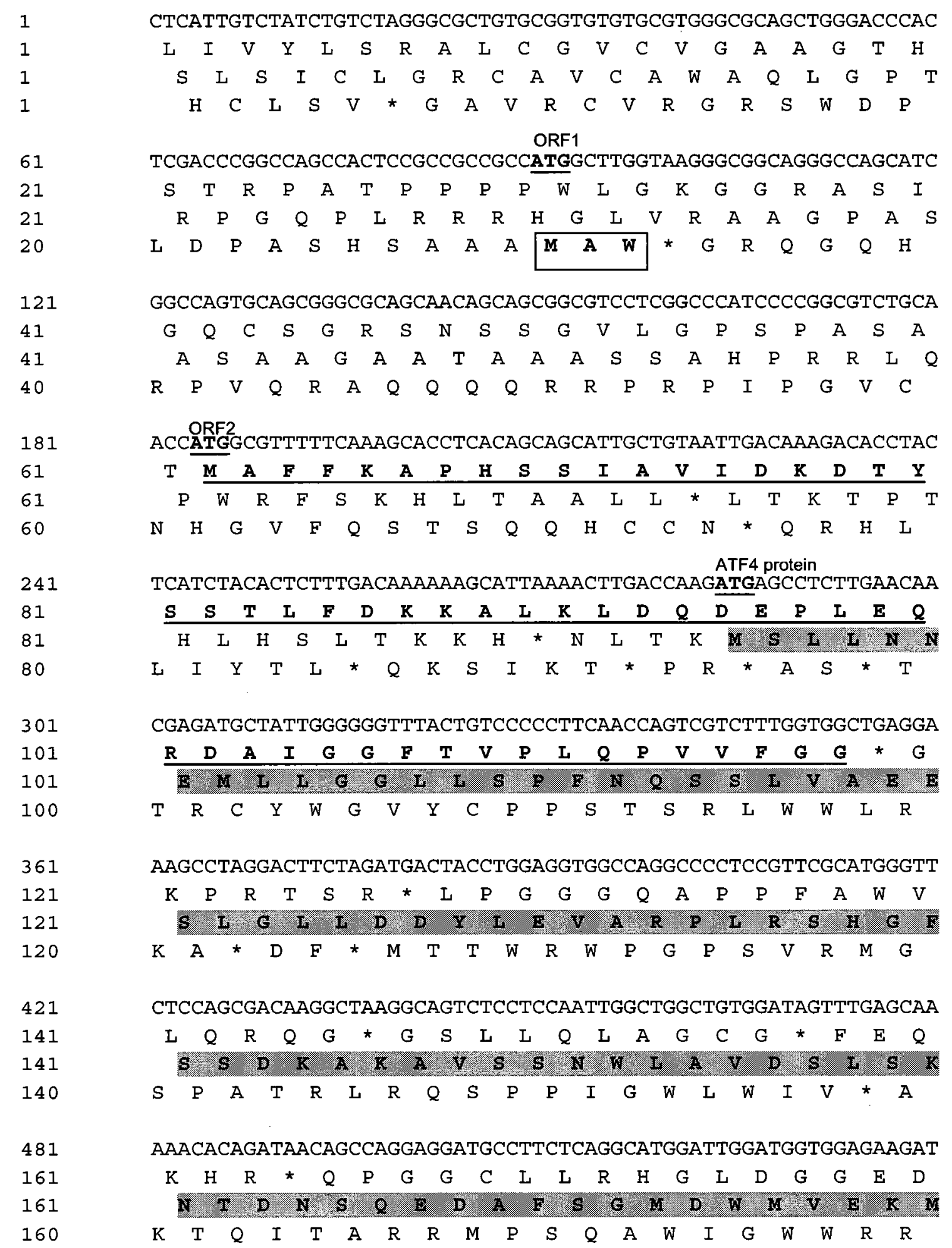


541

181

181

180

601

201

201

200

661

221

221

220

721

241

241

240

781

261

261

260

841

281

281

280

901

301

301

300

961

321

321

320

1021

341

341

340

1081

361

361

360

GGATCTGAAGGAATTTGACTTTGATGCCCTGTTAGGAATGGATGACTTGGAAGCCACCGT

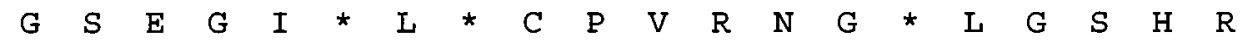

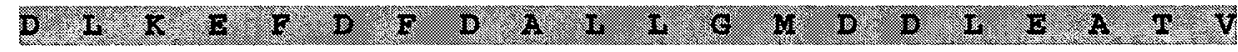
$\begin{array}{llllllllllllllllllll}\mathrm{W} & \mathrm{I} & * & \mathrm{R} & \mathrm{N} & \mathrm{L} & \mathrm{T} & \mathrm{L} & \mathrm{M} & \mathrm{P} & \mathrm{C} & \text { * } & \mathrm{E} & \mathrm{W} & \mathrm{M} & \mathrm{T} & \mathrm{W} & \mathrm{K} & \mathrm{P} & \mathrm{P}\end{array}$ CTCTCCAGATGAGCTCATGGCCACGTTGGAAGACACGTGTGATCTATTAGACCCCTCTAC $\begin{array}{lllllllllllllllllllllll}L & S & R & * & A & H & G & H & V & G & R & H & V & * & S & I & R & P & L & Y\end{array}$

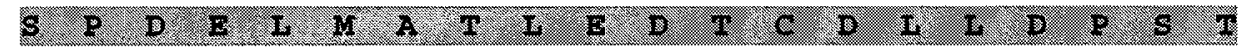
$\begin{array}{lllllllllllllllllllll}S & L & Q & M & S & S & W & P & R & W & K & T & R & V & I & Y & * & T & P & L\end{array}$

CCAGGAAATTCACGACAAAGAACCTCCACTGATAACTGACCCAATTATCAATCACCCTGA

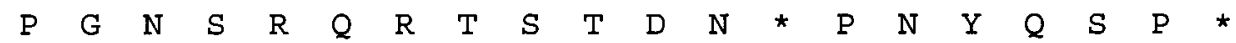

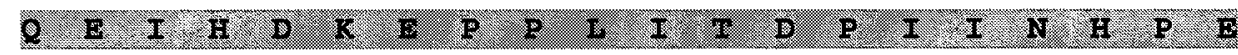

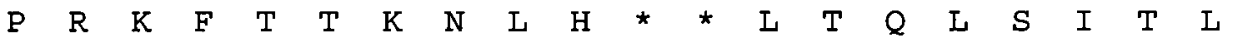
ATCTCCAATTGGAGTAGATCAGGTGGCCTCACTCACCCTCCTTCTGTCTCTTCCCCTCTC $\begin{array}{llllllllllllllllllll}I & S & N & W & S & R & S & G & G & L & T & H & P & P & S & V & S & S & P & L\end{array}$

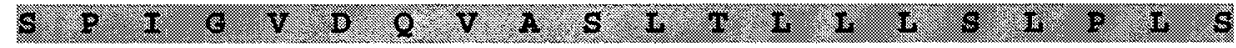
$\begin{array}{llllllllllllllllllll}\mathrm{N} & \mathrm{L} & \mathrm{Q} & \mathrm{L} & \mathrm{E} & * & \mathrm{I} & \mathrm{R} & \mathrm{W} & \mathrm{P} & \mathrm{H} & \mathrm{S} & \mathrm{P} & \mathrm{S} & \mathrm{F} & \mathrm{C} & \mathrm{L} & \mathrm{F} & \mathrm{P} & \mathrm{S}\end{array}$

CCCAGGGTCACTGACTTCCACTCCAGACCATTCTTTTAGTTTAGATCTAGGCAGTGAAGT

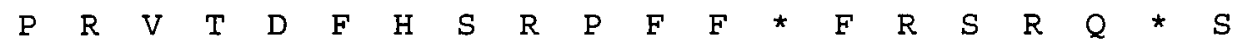

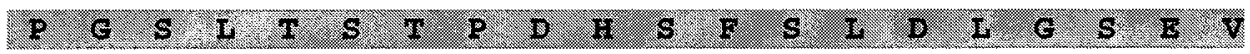

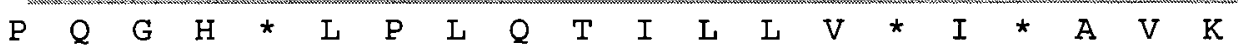
TGATGTACTGGAGGGAGACAGAAAGCCAGAAGCCACCACATTTGTGGTGGTCCCCAAATT $\begin{array}{lllllllllllllllllllll}* & C & T & G & G & R & Q & K & A & R & S & H & H & I & C & G & G & P & Q & I\end{array}$

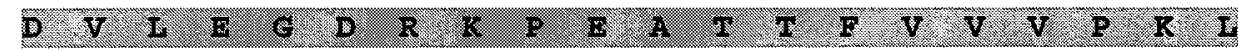
$\begin{array}{llllllllllllllllllll}\mathrm{L} & \mathrm{M} & \mathrm{Y} & \mathrm{W} & \mathrm{R} & \mathrm{E} & \mathrm{T} & \mathrm{E} & \mathrm{S} & \mathrm{Q} & \mathrm{K} & \mathrm{P} & \mathrm{P} & \mathrm{H} & \mathrm{L} & \mathrm{W} & \mathrm{W} & \mathrm{S} & \mathrm{P} & \mathrm{N}\end{array}$ GGAGAAAGAGGAGGAAGCCCACTCAGATGATAGTGGAATATGCATGAGTCCGGAGTCTTA $\begin{array}{lllllllllllllllllllll}G & E & R & G & G & S & P & \text { L } & R & & * & * & \text { W } & \text { N } & \text { M } & \text { H } & \text { E } & S & G & V & L\end{array}$

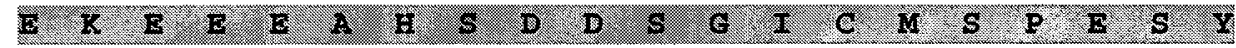
$\begin{array}{lllllllllllllllllllll}W & R & K & R & R & K & P & T & Q & M & I & V & E & Y & A & * & V & R & S & I\end{array}$

TCTAGGGACCCCCCAACACAGTCCAACCACCTCTGTAGAATCTCTCACTGACAGCCAATT

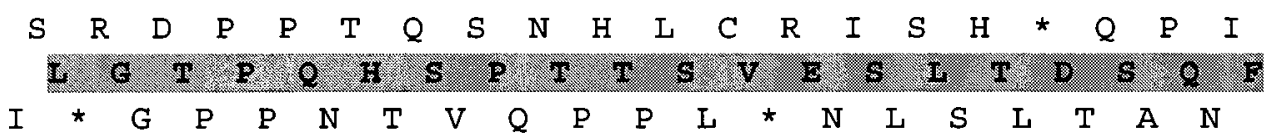
TGCCACAGACCCCATTTGTGATTCTGTGCGGCCTAAACCATATGATCATCCTGCCGAGAA

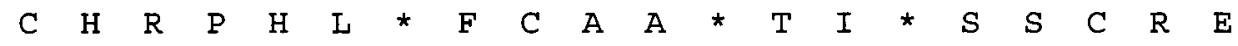

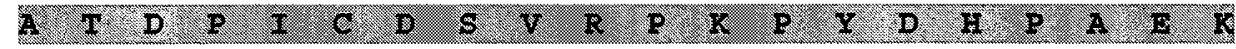
$\begin{array}{lllllllllllllllllllll}\text { L } & \text { P } & \text { Q } & \text { T } & \text { P } & \text { F } & \text { V } & \text { I } & \text { L } & \text { C } & \text { G } & \text { I } & \text { N } & \text { H } & \text { M } & \text { I } & \text { I } & \text { L } & \text { P } & \text { R }\end{array}$ GGTAGTGTCAGCAAAGGTGAAGGGAGAAAAAAGAGCCGATAAGAAACTGAAGAAGATGGA $\begin{array}{lllllllllllllllllllll}G & S & V & S & K & G & E & G & R & K & K & S & R & * & E & T & E & E & D & G\end{array}$

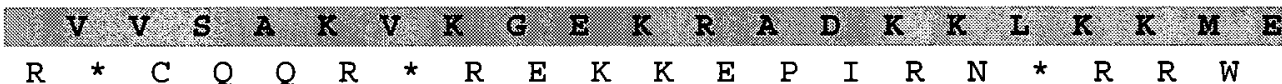




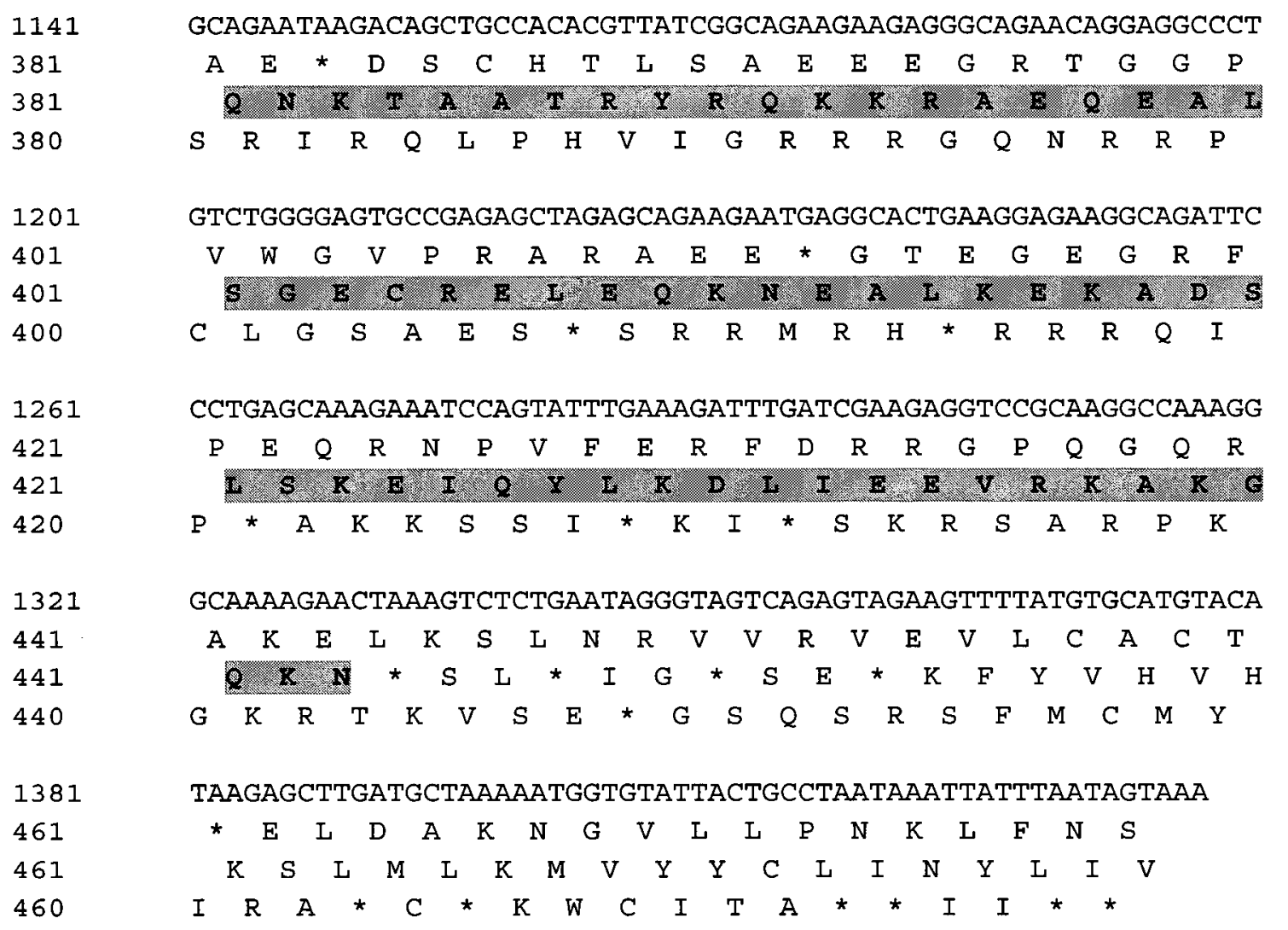

FIG. 2.3. Complete cDNA sequence of atf 4 amplified from liver of $T$. s. elegans and the translated amino acid sequence from three open reading frames.

The start codon for each open reading frame (ORF) is highlighted. ORF 1 is bolded and boxed. ORF2 is bolded and underlined. ORF3 (coding for the ATF4 protein) is bolded and shaded. 
FIG. 2.4
T. $s$.elegans
G. gallus
H. sapiens
$X$. tropicalis
$D$. rerio
T. S .elegans
G. gallus
H. sapiens
$X$. tropicalis
D. rerio
T. $s$.elegans
G. gallus
H. sapiens
$X$. tropicalis
$D$. rerio
T. s .elegans
G. gallus
H. sapiens
$X$. tropicalis
D. rerio
T. s.elegans
G. gallus
$H$. sapiens
$X$. tropicalis
D. rerio
T. S .elegans
G. gallus
H. sapiens
$X$. tropicalis
D. rerio
T. S . elegans
G. gallus
H. sapiens
$X$. tropicalis
$D$. rerio
T. $s$.elegans
G. gallus
H. sapiens
$X$. tropicalis
$D$. rerio
T. s .elegans
G. gallus
H. sapiens
$X$. tropicalis
D. rerio

. . MSLLNNEMLLGGLLSPFNQSSLVAEESLGLLDDYLEV 37

...-------es.--s-pcs---------- 36

mte--f-ss-v-v-d-m---d--g-g---.--.-.-- 40

........---d------f-a--k----e-ef-- 30

...ms-cvgdagal--g-wp...-t-dpqgp---qde-s 33

ARPLRSHGFSSDKAKAVS ............ SNWLAVDSLS 65

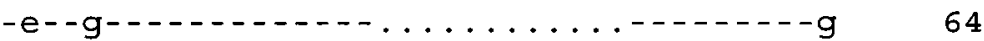

$-k h f k p--------g-\ldots \ldots \ldots \ldots . \mathrm{e}----g-\mathrm{g} \quad 68$

pgy-l-q------dgv.........dl-pyvesl 58

svleg-wsp--sslssf-ppasgdapsell-lswqpsdvl 73

KNTDNSQEDAFSGMDWMVEKMDLKEFDFDALLGMDDLEAT 105

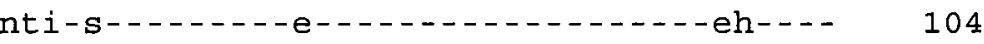

spsn--k-----t---1---------1-----i----tm 108

gsfhg....---.----.-----l-s-f-le---s- 95

lteqstag-v-p-----t--1--nd--1-s-i-sc-s-ds 113

VSPDELMATLEDTCDLLD. PSTQEIHDKEPPLITDPIINH 144 - - - - - - - - - - - fn-t---f-n-----p-l-t-1 144

..--d-lt--d----fa.-lv--tn.-q--qtvn--ghl 144

i----ts---s--f-e.dpphaavighfvpspe-vsl. 133

pgsp-dllacl-ssmd--. ldslpfgsadld-plgldlpl 152

PESPIGVDQVASLTLLLSLPLSPGSLTSTPDHSFSLDLGS 184

- - - - a-pm-p-ss-w-f--------v--.---e--- 184

- - - ltkp----pf-f-qp------v-s-------e--- 184

----lda----pfspd-tas--.....s-----i--v-- 168

--eik.........-e----apsvps-peeapq-eht 180

EVDVLE . . GDRKPEATTF . VVV. PKLEKEEEAHSD.DSGI 219

$-----. .-e--q-g p--1--i . t-s----n---.---220$

---it-..----dy-ay. -ami-qci---dtp--n-..- 221

----a-..dek-ss--yt.lei.1-c---dssdn-..-- 202

--p--hpa-imlslspsh.i--.lltp---qni--.c-ds 217

CMSPESYLGTPQHSPTTSVESLTDSQFATDPICDSVRPKP 259

- - - d- - - - - - - n-lg-pn-np-p--aa-g---s-- 260

- - - - - - s----s-rg. -pnr-lpspgvl-g-a--- 260

s--..--es--q--s-vecysspqssles-v..-e---- 239

d.-gi-vs-s-a-qsdleps-rakpys.......--d- 247

YDHPAEKV . VSAKVK. . GEKRADKKLKKMEQNKTAATRY 295

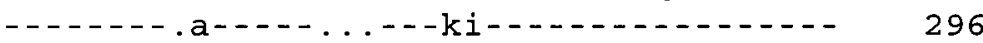

$--\mathrm{p}-\mathrm{g}--\mathrm{m} .-\mathrm{a}----\ldots .--1 .--------------295$

--1-ckdrd-m----vag-qp-v---k------------ 279

eas--1-g.rvktss...-apkve------------- 283

RQKKRAEQEALSGECRELEQKNEALKEKADSLSKEIQYLK 335

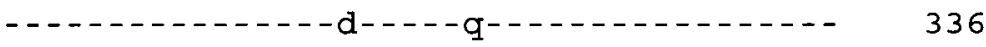

- - - - - - - - t---k---k----- r----a----- 335

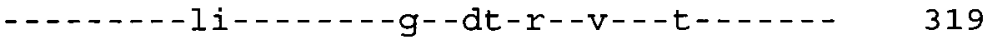

- - - - v---s-ns--s---k--re-s------r----r 323 
$\begin{array}{lll}\text { T. S .elegans } & \text { DLIEEVRKAKGQKN.... } & 349 \\ \text { G. gallus } & ------- \text {-vkrarvpe } & 354 \\ \text { H. sapiens } & ------- \text {-k-rvp.. } & 351 \\ \text { X. tropicalis } & ---a-- \text {---skrakss. } & 336 \\ \text { D. rerio } & --1-- \text {-t--qr-skr.. } & 339\end{array}$

FIG. 2.4. Complete amino acid sequence of ATF4 from T. s. elegans.

The turtle sequence is compared with ATF4 sequences from human (Homo sapiens), chicken (Gallus gallus), western clawed frog (Xenopus tropicalis), and zebrafish (Danio rerio). Accession numbers are NP_990211 for chicken, NP_877962 for human, NP_001004785 for frog and NP_998398 for zebrafish. Dashes replace amino acid residues that are identical with $T$. s. elegans whereas spacer dots indicate residues that are missing in the sequence. 
FIG. 2.5

A)
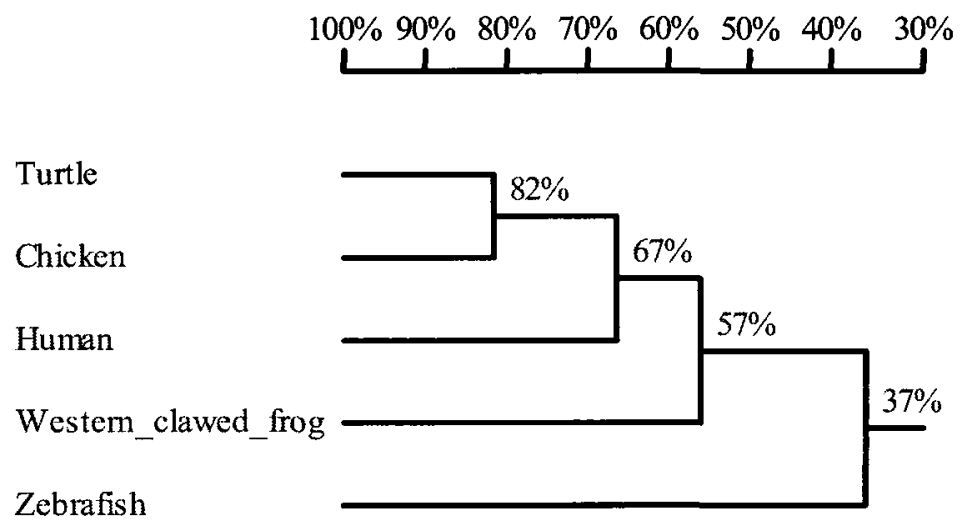

B)

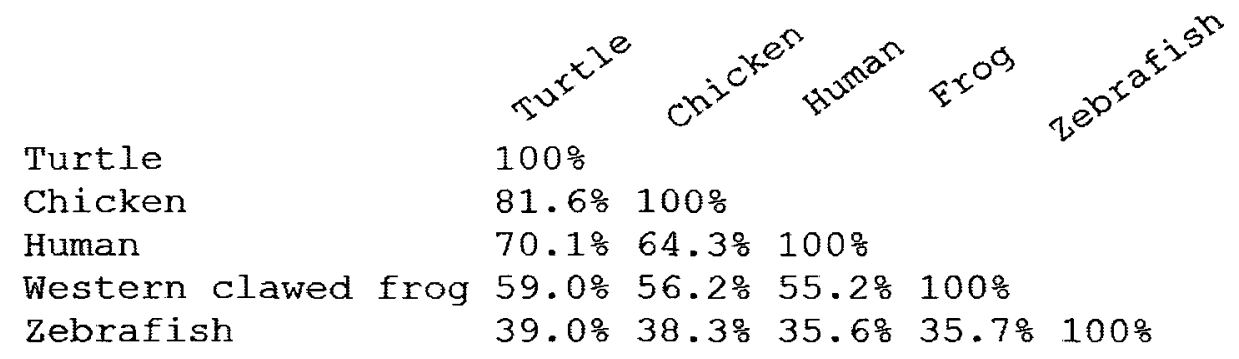

FIG. 2.5 .

(A) Homology tree produced from an alignment of the T. s. elegans ATF4 protein sequences with ATF4 from human (Homo sapiens), chicken (Gallus gallus), western clawed frog (Xenopus tropicalis), and zebrafish (Danio rerio). Accession numbers are as above. The percentage values correspond to the shared identity between the corresponding species.

(B) Homology matrix showing amino acid identity of the T. s. elegans ATF4 sequence compared with ATF4 from the other vertebrates. 
FIG. 2.6

A)
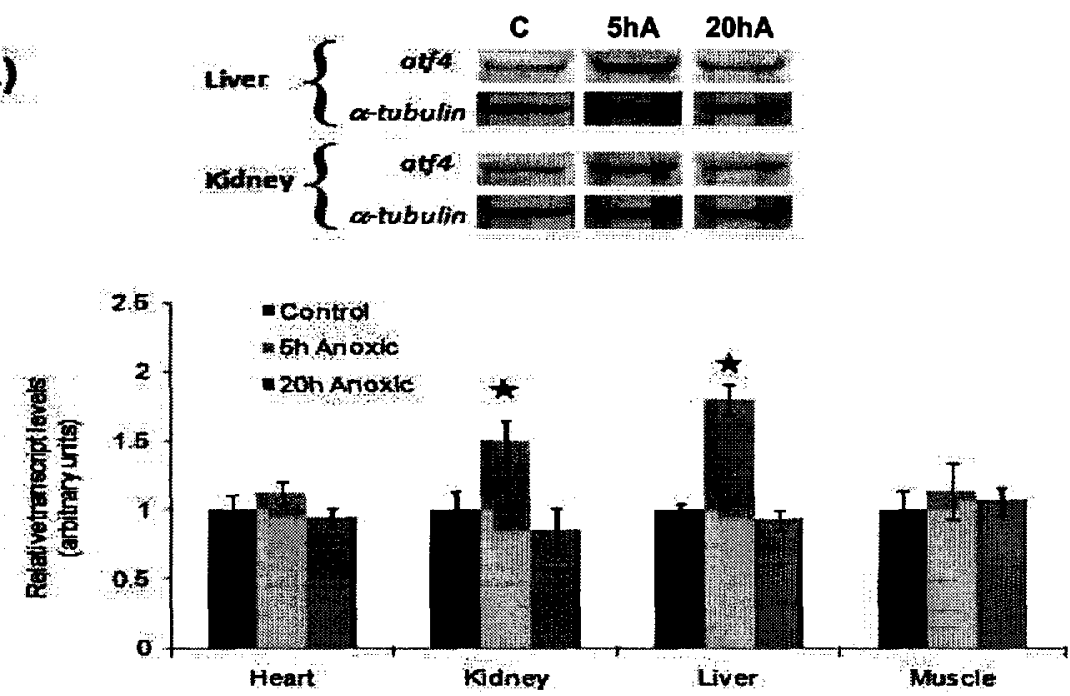

B)

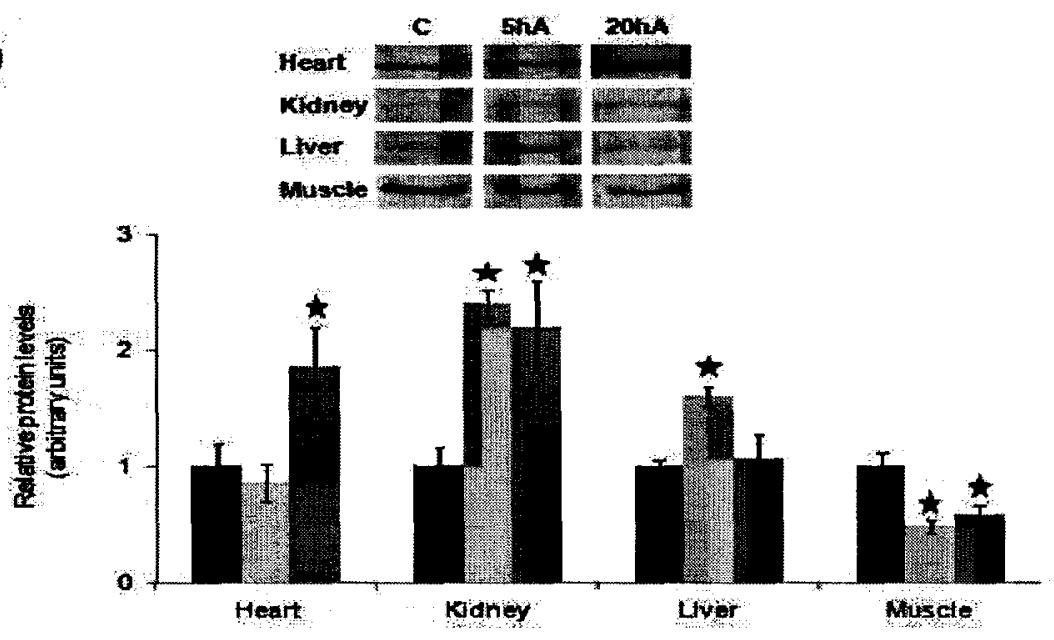

FIG. 2.6. Effect of 5 and $20 \mathrm{~h}$ of anoxic submergence on ATF4 expression in T. $s$. elegans tissues.

(A) Changes in atf4 transcript levels in response to anoxia, as determined by RT-PCR. Representative atf 4 amplicons with corresponding $\alpha$-tubulin bands are shown along with a histogram showing the mean normalized transcript levels under control and anoxic conditions. Bands for atf4 were normalized against the corresponding $\alpha$-tubulin band from the same sample.

(B) Changes in ATF4 protein levels in response to anoxia, as determined by Western immunoblotting. Representative western immunoblot bands are shown along with a histogram with normalized protein levels under control and anoxic conditions. Data are means \pm SEM, $n=3-5$ independent trials. ${ }^{*}$ Significantly different from the corresponding control, $P<0.05$. 
FIG. 2.7

A)

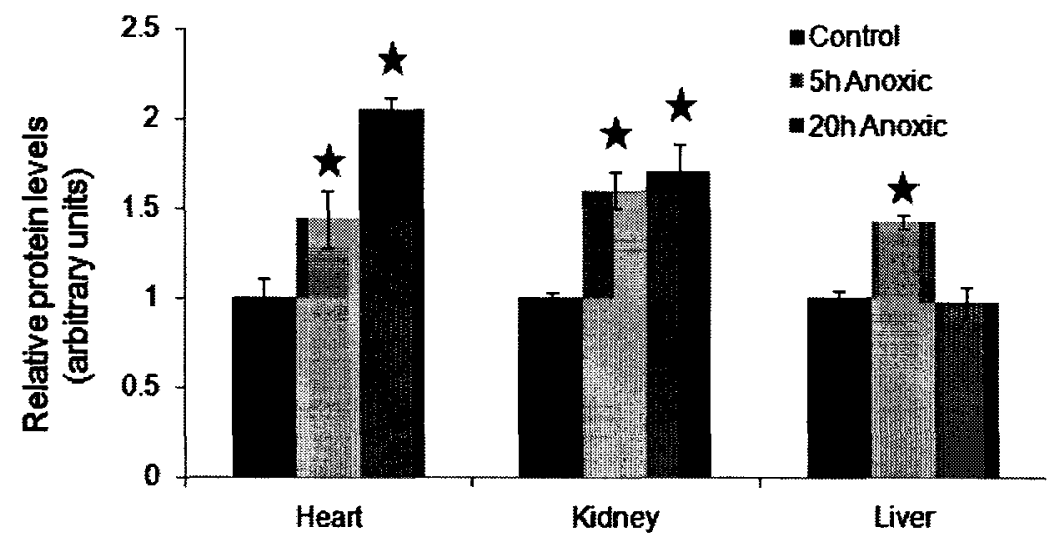

B)

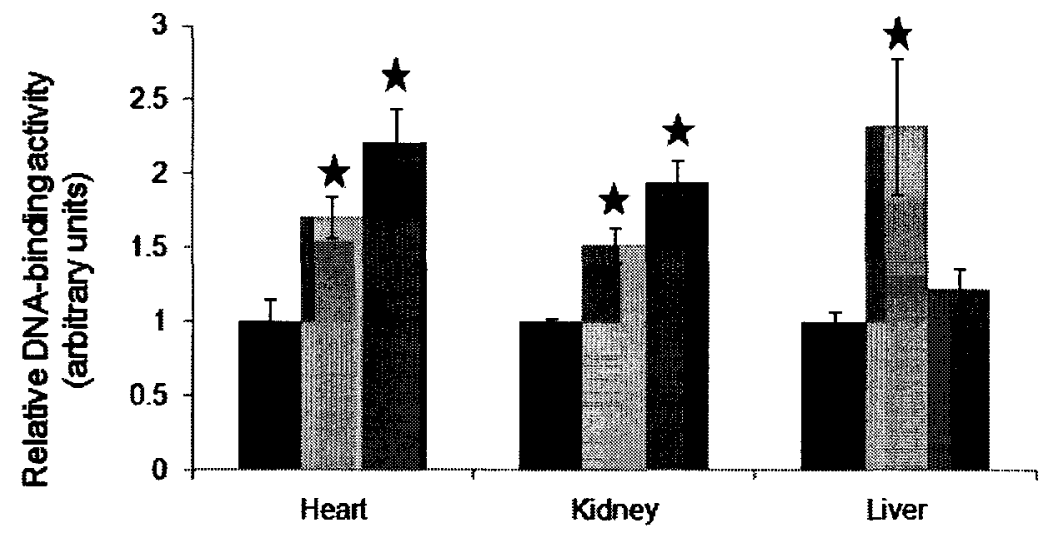

FIG. 2.7. Effect of 5 and $20 \mathrm{~h}$ of anoxic submergence on ATF4 activation in $T$. $s$. elegans tissues.

(A) Changes of ATF4 protein levels in the nuclear fraction of turtle tissues in response to anoxia, as determined by Western immunoblotting.

(B) Changes in the DNA-binding activity of nuclear ATF4 in response to anoxia, as determined by a transcription factor binding assay. Data are means \pm SEM, $n=3-4$ independent trials for liver and semi-independent trials for heart and kidney. * Significantly different from the corresponding control, $P<0.05$. 


\section{FIG. 2.8}
A)
1
AGCGCCTCCGCAATTGTTCCCTGTTTGTCTCCTACAGCGTCACTGGGGTTTGAGGATTTC
1
$\begin{array}{lllllllllllllllllllll}S & A & S & A & I & V & P & C & \text { L } & S & P & T & A & S & L & G & F & E & D & F\end{array}$
61
ACAAATCTAACCCCACTGGTGAAGGAGGAATTGAGATTTGCCATCCAAAATAAGTGCCTA
21
$\begin{array}{llllllllllllllllllll}T & N & L & T & P & L & V & K & E & E & L & R & F & A & I & Q & N & K & C & L\end{array}$
121
TCCCACAGGATGTCTTCCACATTGGATACAGTGACAGTGTTGGAAAGACCTGTTGAAATG
41
$\begin{array}{llllllllllllllllllll}S & H & R & M & S & S & T & L & D & T & V & T & V & \text { L } & \text { E } & R & \text { P } & \text { V } & \text { E } & \text { M }\end{array}$
181
TCTGTTCTGAAAGCAGAGTTTTCACCCCAAGAAGATGAAAGGAAAAAGAGAAGAAGGGAA
61
$\begin{array}{llllllllllllllllllll}S & V & L & K & A & E & F & S & P & Q & E & D & E & R & K & K & R & R & R & E\end{array}$
241
81
AGGAACAAAATTGCAGCTGCAAAATGCCGAAACAAAAAGAAGGAGAAAACAGAATGTTTG
301
$\begin{array}{llllllllllllllllllll}R & N & K & I & A & A & A & K & C & R & N & K & K & K & E & K & T & E & C & L\end{array}$
101
CAGAAAGAATCAGAAAAGTTGGAAACTATCAAC
$\begin{array}{lllllllllll}Q & K & E & S & E & K & L & E & T & I & N\end{array}$

B)
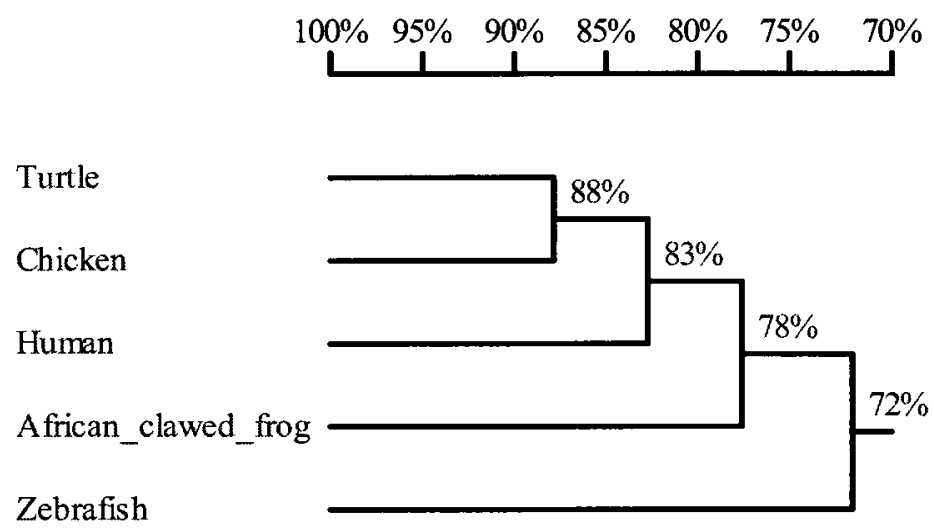
FIG. 2.8. Nucleotide and deduced amino acid sequence of $T$. s. elegans ATF3.

(A) A partial cDNA sequence of atf3 was retrieved and is shown with the corresponding amino acid sequence. Nucleotides and amino acids are numbered on the left. The nucleotide sequence was 333 nucleotides long and encoded 111 amino acids. This sequence covered amino acids $15-125$ of the chicken ATF3 sequence, representing $61 \%$ of the total ATF3 protein.

(B) Homology tree produced from an alignment of the partial T. s. elegans ATF3 protein sequence with ATF3 from human (Homo sapiens), chicken (Gallus gallus), African clawed frog (Xenopus laevis) and zebrafish (Danio rerio). Accession numbers are XP_419429 for chicken, NP_001665 for human, NP_001087487 for frog and NP_957258 for zebrafish. The percentage values correspond to the shared identity among the corresponding species. 
FIG. 2.9
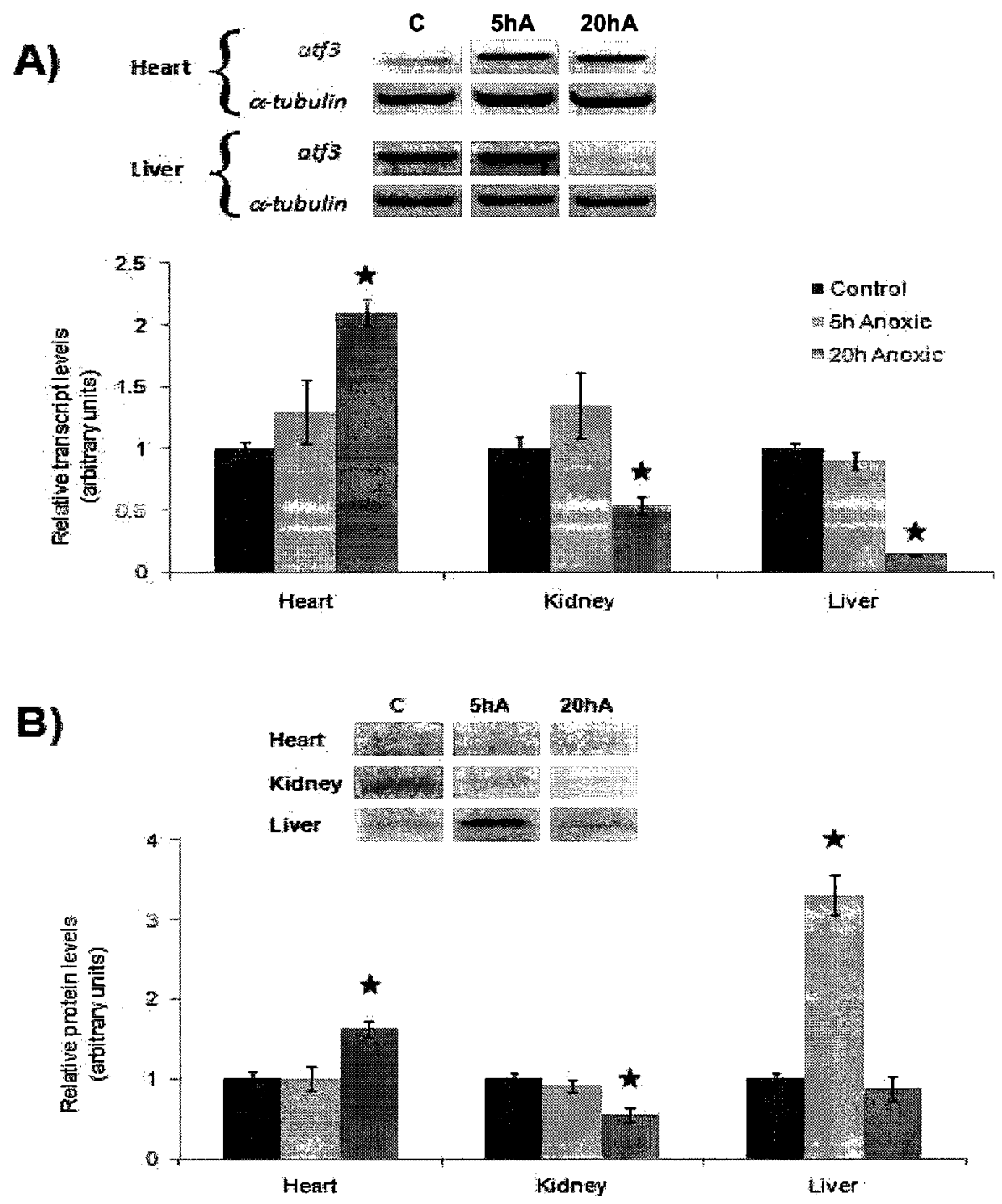

FIG. 2.9. Effect of 5 and $20 \mathrm{~h}$ of anoxic submergences on ATF3 expression in $T . s$. elegans tissues.

(A) Changes in atf 3 transcript levels in response to anoxia, as determined by RT-PCR. Representative aft 3 amplicons with corresponding $\alpha$-tubulin bands under control and anoxic conditions are shown. The histogram shows the normalized transcript levels under control and anoxic conditions; bands were normalized against the corresponding $\alpha$ tubulin bands from the same sample.

(B) Changes in ATF3 protein levels in response to anoxia, as determined by Western immunoblotting. Representative western immunoblot bands are shown along with a histogram showing normalized protein levels under control and anoxic conditions. Data are means \pm SEM, $n=3-5$ independent trials. * Significantly different from the corresponding control, $P<0.05$. 
FIG. 2.10
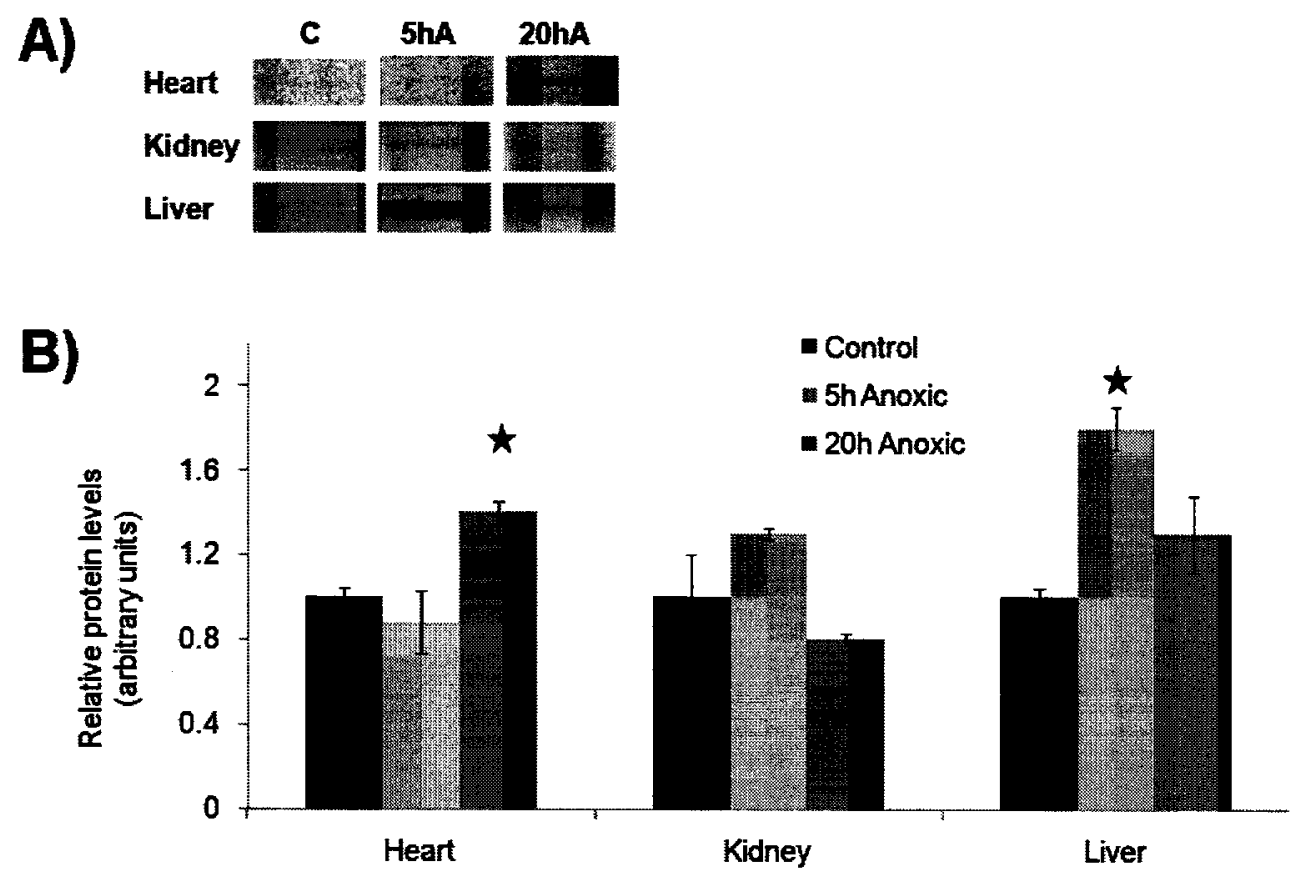

FIG. 2.10. Effect of 5 and $20 \mathrm{~h}$ of anoxic submergence the expression of GADD34 in T. s. elegans tissues as determined by Western immunoblotting.

(A) Representative western immunoblots showing changes in GADD34 expression under control and anoxic conditions.

(B) Histogram showing normalized protein levels; data are means \pm SEM, $n=3-5$ independent trials. * Significantly different from the corresponding control, $P<0.05$. 
FIG. 2.11
A)
GAGAACACCGTGTTCGATGCCAAGCGCCTCATTGGCCGCACGTGGAACGACCCGTCGGTG $\begin{array}{llllllllllllllllllllll}\text { E } & \mathrm{N} & \mathrm{T} & \mathrm{V} & \mathrm{F} & \mathrm{D} & \mathrm{A} & \mathrm{K} & \mathrm{R} & \mathrm{L} & \mathrm{I} & \mathrm{G} & \mathrm{R} & \mathrm{T} & \mathrm{W} & \mathrm{N} & \mathrm{D} & \mathrm{P} & \mathrm{S} & \mathrm{V}\end{array}$ CAGCAGGATATCAAGTACCTGCCGTTCAAGGTTGTTGAAAAGAAGACCAAGCCATATATA $\begin{array}{llllllllllllllllllll}Q & Q & D & I & K & Y & L & P & F & K & V & V & E & K & K & T & K & P & Y & I\end{array}$ CAAGTTGATGTTGGTGGTGGACAGACAAAGACATTTGCTCCAGAAGAAATTTCTGCTATG $\begin{array}{lllllllllllllllllllll}Q & V & D & V & G & G & G & Q & T & K & T & F & A & P & E & E & I & S & A & M\end{array}$ GTTTTGACAAAGATGAAGGAAACTGCTGAGGCCTATTTAGGAAAGAAGGTTACCCATGCT 61 $\begin{array}{llllllllllllllllllll}V & L & T & K & M & K & E & T & A & E & A & Y & L & G & K & K & V & T & H & A\end{array}$ GTTGTCACTGTCCCAGCTTATTTCAATGATGCTCAGCGTCAAGCTACAAAGGATGCTGGT 81 $\begin{array}{lllllllllllllllllllll}\mathrm{V} & \mathrm{V} & \mathrm{T} & \mathrm{V} & \mathrm{P} & \mathrm{A} & \mathrm{Y} & \mathrm{F} & \mathrm{N} & \mathrm{D} & \mathrm{A} & \mathrm{Q} & \mathrm{R} & \mathrm{Q} & \mathrm{A} & \mathrm{T} & \mathrm{K} & \mathrm{D} & \mathrm{A} & \mathrm{G}\end{array}$ ACCATTGCTGGATTGAATGTAATGAGGATCATCAATGAACCTACTGCTGCTGCCATTGCT TATGGACTGGATAAGCGGGAGGGTGAGAAGAACATCCTTGTATTTGATCTAGGCGGTGGA $\begin{array}{llllllllllllllllllll}Y & G & L & D & K & R & E & G & E & K & N & I & \text { L } & \text { V } & \text { F } & \text { D } & \text { L } & G & G & G\end{array}$ ACCTTTGATGTCTCCCTCCTCACAATTGATAATGGTGTCTTTGAAGTTGTGGCTACAAAT $\begin{array}{llllllllllllllllllll}\text { T } & \text { F } & \text { D } & \text { V } & \text { S } & \text { L } & \text { L } & \text { T } & \text { I } & \text { D } & \text { N } & \text { G } & \text { V } & \text { F } & \text { E } & \text { V } & \text { V } & \text { A } & \text { T } & \text { N }\end{array}$ GGAGATACTCACTTGGGCGGAGAGGACTTTGACCAACGTGTTATGGAACACTTTATCAAG $\begin{array}{llllllllllllllllllll}G & D & T & H & \text { L } & G & G & E & D & F & D & Q & R & V & M & E & H & F & I & K\end{array}$ 
B)
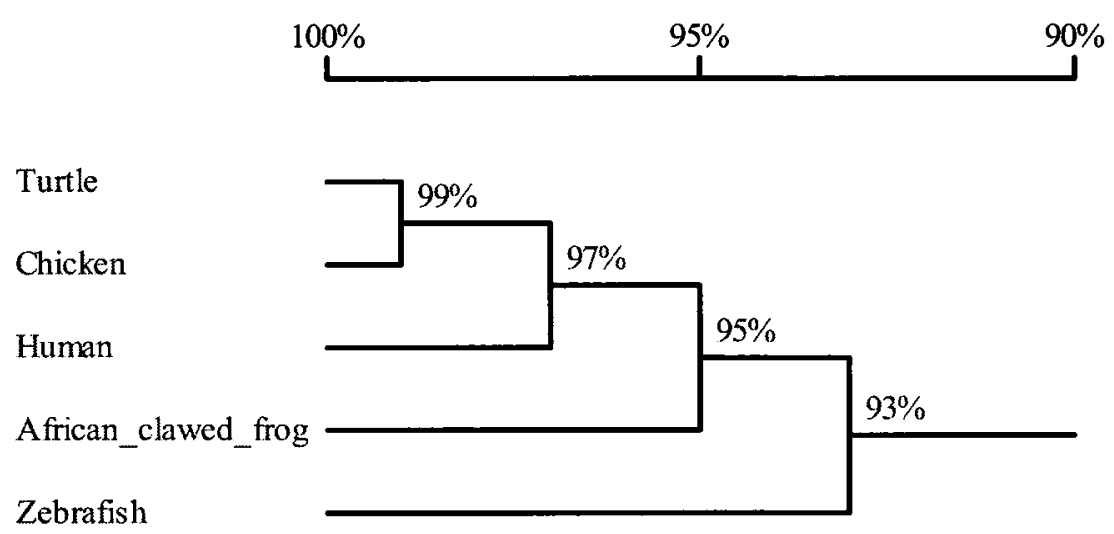

FIG. 2.11. Nucleotide and deduced amino acid sequence of $T$. s. elegans GRP78.

(A) A partial cDNA sequence of $g r p 78$, with the corresponding amino acid sequence was retrieved. Nucleotides and amino acids are numbered on the left. The nucleotide sequence was 837 nucleotides in length, and encoded 279 amino acids. This sequence corresponded to amino acids 87-365 of the chicken GRP78 sequence, representing $43 \%$ of the total GRP78 protein.

(B) Homology tree produced from an alignment of the partial T. s. elegans ATF3 protein sequence with ATF3 from human (Homo sapiens), chicken (Gallus gallus), African clawed frog (Xenopus laevis) and zebrafish (Danio rerio). Accession numbers are NP_990822 for chicken, NP_005338 for human, NP_001080064 for frog and AAH63946 for zebrafish. The percentage values correspond to the shared identity among the corresponding species. 
FIG. 2.12

A)
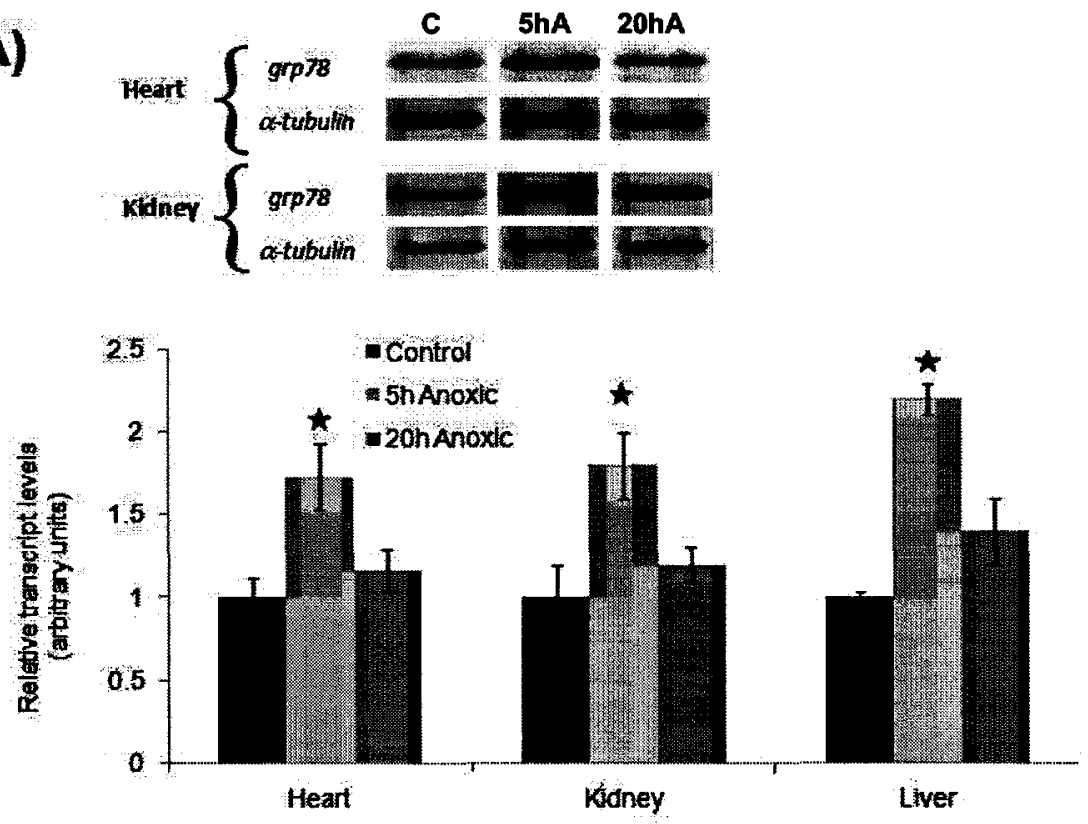

B)

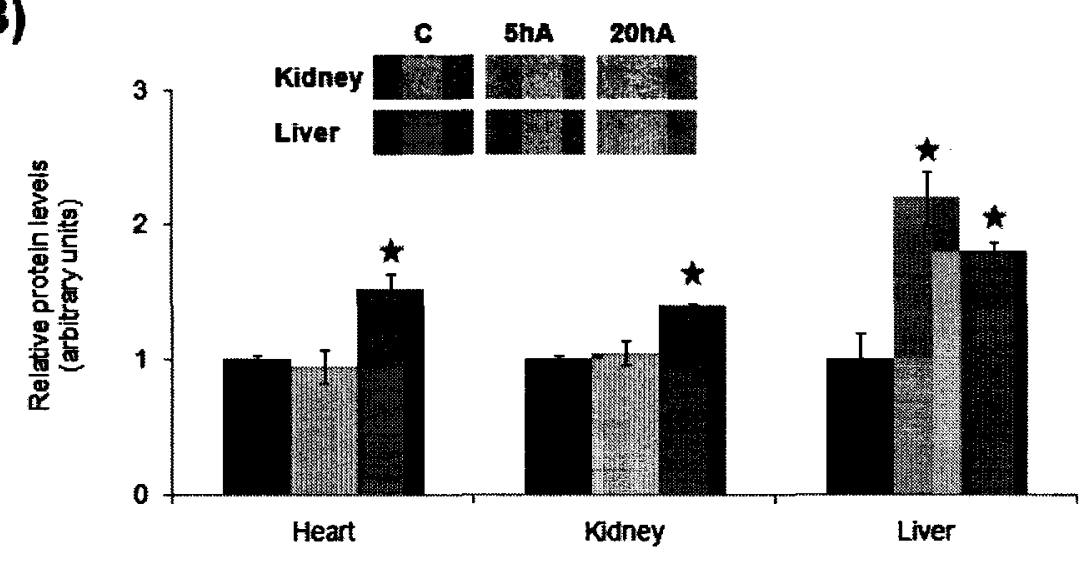

FIG. 2.12. Effect of 5 and $20 \mathrm{~h}$ of anoxic submergence on GRP78 expression in $T$. $s$. elegans tissues.

(A) Changes in $\operatorname{grp} 78$ transcript levels in response to anoxia, as determined by RT-PCR. Representative $\operatorname{grp} 78$ amplicons with corresponding $\alpha$-tubulin bands under control and anoxic conditions are shown. The histogram shows the normalized transcript levels under control and anoxic conditions; bands were normalized against the corresponding $\alpha$ tubulin bands from the same sample.

(B) Changes in GRP78 protein levels in response to anoxia, as determined by Western immunoblotting. Representative western bands are shown along with a histogram showing normalized protein levels under control and anoxic conditions. Data are means \pm SEM, $n=3-5$ independent trials. * Significantly different from the corresponding control, $P<0.05$. 
FIG. 2.13

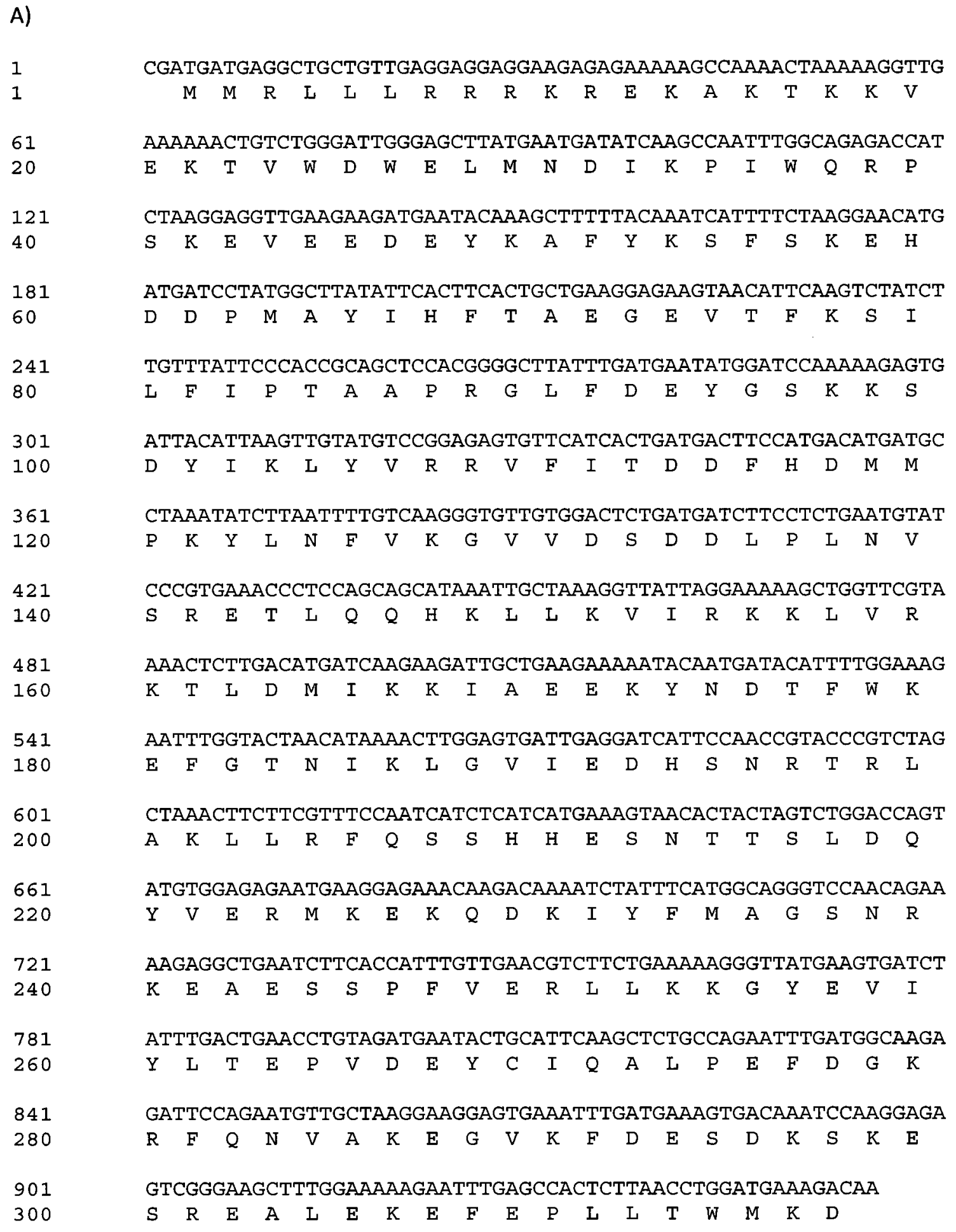


B)
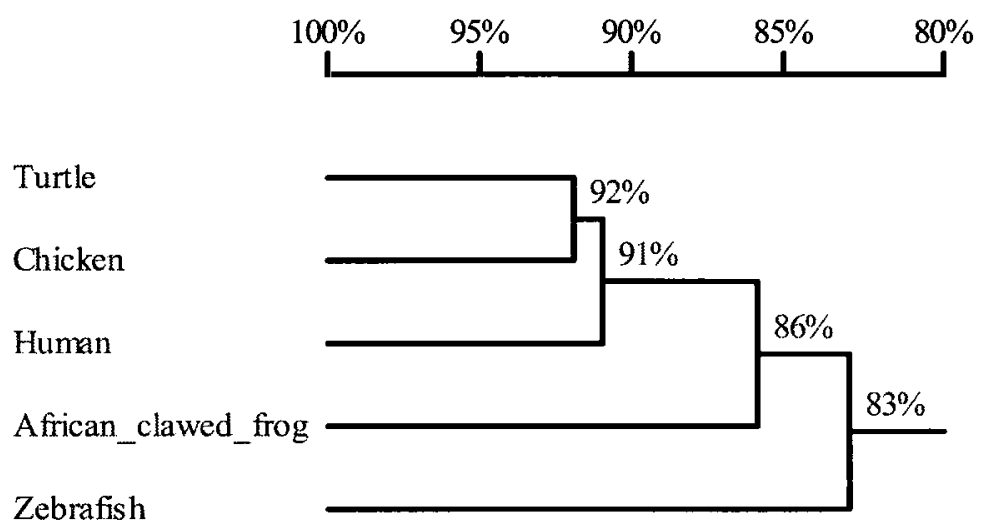

FIG. 2.13. Nucleotide and deduced amino acid sequence of $T$. s. elegans GRP94.

(A) A partial cDNA sequence of grp94 was retrieved and is shown with the corresponding amino acid sequence. Nucleotides and amino acids are numbered on the left. The nucleotide sequence was 955 nucleotides in length and encoded 317 amino acids. This protein covered amino acids 329-623 of the chicken GRP94 sequence, about $40 \%$ of the total GRP94 protein.

(B) Homology tree produced from an alignment of the partial T. s. elegans GRP94 protein sequence with GRP94 from human (Homo sapiens), chicken (Gallus gallus), African clawed frog (Xenopus laevis) and zebrafish (Danio rerio). Accession numbers are NP_989620 for chicken, NP_003290 for human, NP_001084280 for frog and NP_937853 for zebrafish. The percentage values correspond to the shared identity among the corresponding species. 
FIG. 2.14
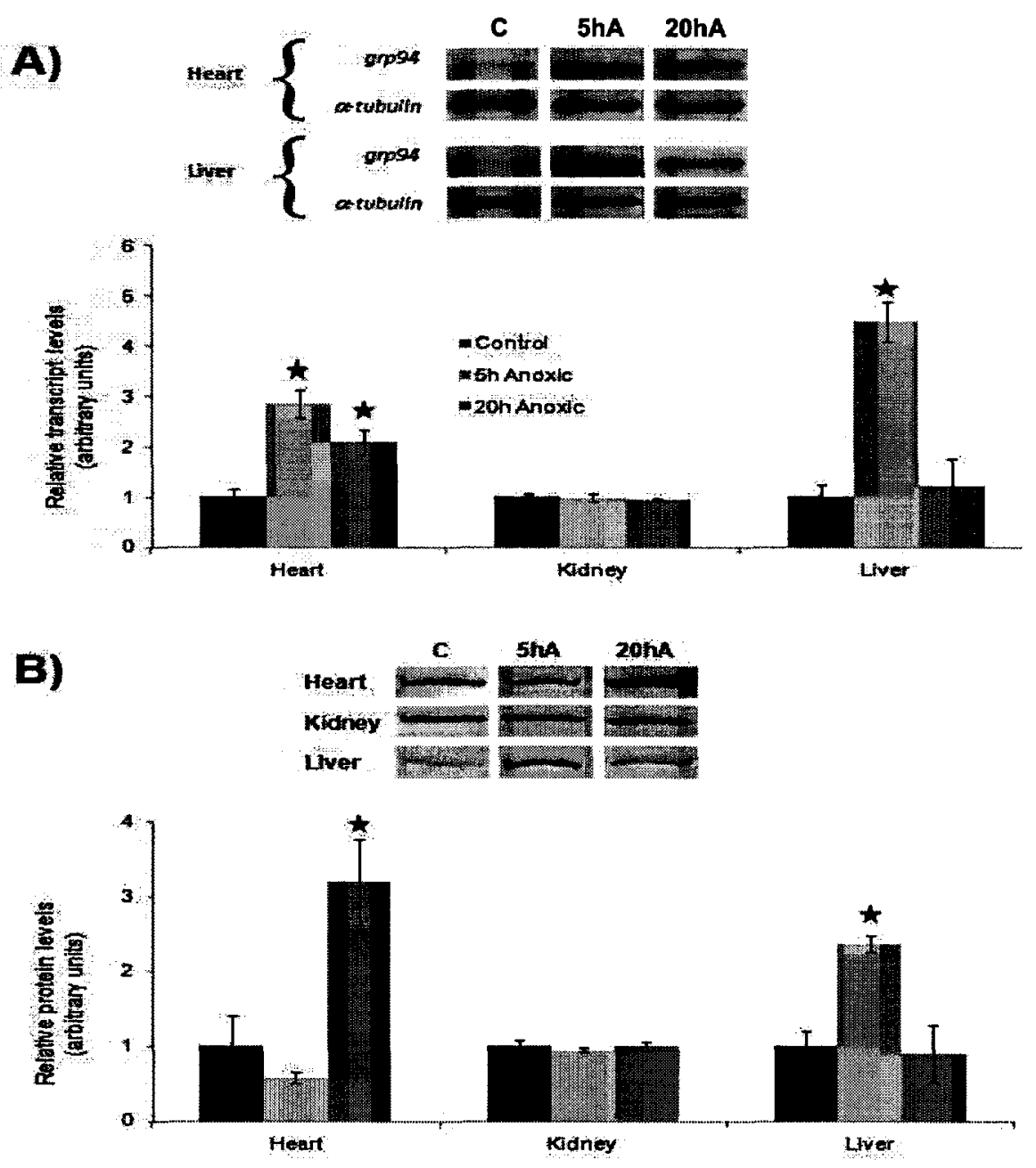

FIG. 2.14. Effect of 5 and $20 \mathrm{~h}$ of anoxic submergence on GRP94 expression in $T$. $s$. elegans tissues.

(A) Changes in grp94 transcript levels in response to anoxia, as determined by RT-PCR. Representative grp 94 amplicons with corresponding $\alpha$-tubulin bands are shown along with a histogram showing mean normalized transcript levels for control and anoxic conditions. Bands were normalized against the corresponding $\alpha$-tubulin bands from the same sample.

(B) Changes in GRP94 protein levels in response to anoxia, as determined by Western immunoblotting. Representative western bands are shown along with a histogram showing normalized protein levels under control and anoxic conditions. Data are means \pm SEM, $n=3-5$ independent trials. * Significantly different from the corresponding control, $P<0.05$. 


\section{CHAPTER 3}

\section{Molecular mechanisms of anoxia tolerance: a role for $\mathrm{NF}-\mathrm{\kappa B}$ \\ (Published in Gene)}




\subsection{INTRODUCTION}

As discussed in chapter 2, anoxia can be a stressful event for $T . s$. elegans tissues, and it appears that there is a requirement for activation of cytoprotective mechanisms to avoid cellular damage during bouts of anoxia and reoxygenation. Another potentially important transcription factor that has risen to prominence as an important regulator of the cellular stress response is NF- $\mathrm{KB}$. NF- $\mathrm{\kappa B}$ is an oxygen-responsive transcription factor that can become activated in response to a variety of stimuli and promote the expression of many target genes, including genes involved in the immune response, antioxidant defenses, cell growth and differentiation and apoptosis (reviewed by Pahl, 1999). NF-kB is homo- or hetero- dimeric, and is composed of proteins containing the Rel Homology domain (Chen and Ghosh, 1999). The NF- $\kappa B$ family is comprised of five members, p50 and its precursor p105 (also known as NF- $\mathrm{kB1}$ ), p52 and its precursor p100 (also known

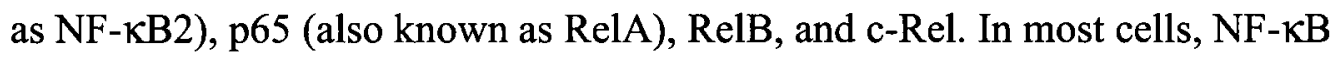
transcription complexes are present in a latent, inactive state in the cytoplasm where they

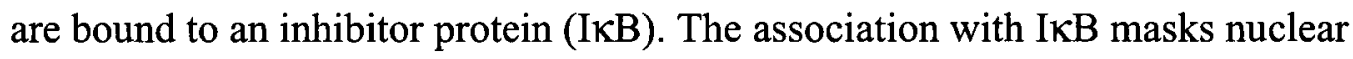
localization sites on NF- $\mathrm{kB}$ and prevents it from entering the nucleus. Various stimuli induce phosphorylation of $I \kappa \mathrm{B}$, targeting it for ubiquitination and degradation, and freeing NF- $\mathrm{KB}$ to translocate to the nucleus and mediate expression of downstream genes (FIG.3.1; Karin and Ben Neriah, 2000; Read et al., 2000).

Because of its rapid response to stress and its important role as a mediator of many cellular processes, the NF- $\mathrm{BB}$ pathway has been studied extensively in mammalian ischemia/reperfusion injury (Cummins and Taylor, 2005; Luedde and Trautwein, 
2006; Nichols, 2004; Ridder and Schwaninger, 2009). The role of this transcription factor, however, is not entirely clear, with some studies demonstrating the beneficial effects of NF-KB activation (Kuboki et al., 2007; Misra et al., 2003), while others suggest a more detrimental role (Luedde et al., 2005; Fan et al., 2004; Onai et al., 2004). Examination of this pathway in an anoxia-tolerant model has the advantage that being well-adapted to anoxia, this animal is not expected to activate any pathways that might cause damage to its organs.

Because of the important role of NF- $\mathrm{KB}$ in the cellular stress response, I hypothesized that this transcription factor will become activated in response to anoxia in T. s. elegans. The present chapter explores the activation of this transcription factor in the turtle liver. To examine the NF- $\kappa \mathrm{B}$ response to anoxia, I measured the transcript and protein levels of the proteins that make up NF- $\mathrm{kB}, \mathrm{p} 50$ and p65. Nuclear localization, as well as NF- $\mathrm{BB}$ DNA-binding activity were also measured. In addition, the transcript levels of selected NF- $\mathrm{KB}$ target genes, including those involved in antioxidant defenses as well as anti-apoptotic proteins were measured. 


\subsection{MATERIALS AND METHODS}

\subsubsection{Animals}

All animal experiments were performed as described in chapter 2. Liver was the only tissue used for most analyses.

\subsubsection{Total RNA Extraction and $c D N A$ synthesis}

RNA extraction and cDNA synthesis were performed as described in Chapter 2.

\subsubsection{Primers}

Forward and reverse primers for $N F-k B N F K B 1$ (p50) and $p 65$, as well as $M n S O D, C u / Z n S O D, B c l-x L$ and $B c l-2$ were designed from conserved regions in these genes based on DNA alignment with other vertebrate species. The ferritin heavy chain (Fth1) primers were designed based on partial soft-shell turtle (Pelodiscus sinensis) mRNA sequence for Fthl available from GenBank (ID: AY998625). Primer sequences are listed in table 3.1.

\subsubsection{PCR amplification}

PCR was performed as described in Chapter 2. The PCR program was as follows: $7 \mathrm{~min}$ at $94^{\circ} \mathrm{C}$ followed by 36 cycles of $1 \mathrm{~min}$ at $94^{\circ} \mathrm{C}, 1 \mathrm{~min}$ at a predetermined 
annealing temperature $\left(54^{\circ} \mathrm{C}\right.$ for all genes except $\left.62.5^{\circ} \mathrm{C} B c l-x L\right)$, and 1.5 min at $72^{\circ} \mathrm{C}$. The final step was $72^{\circ} \mathrm{C}$ for 10 min. PCR products were separated on a $1 \%$ agarose gel, stained with ethidium bromide, visualized using the ChemiGenius imaging system (Syngene, Frederick, MD, USA) under UV light and quantified using the GeneTools program. The bands from the most dilute cDNA sample that gave visible product were used for quantification purposes to make sure that the products had not reached amplification saturation.

\subsubsection{Sequencing}

The PCR products were sequenced by DNA Landmarks (St.-Jean-sur-Richelieu, QC). The sequences were verified as encoding the correct genes using the program BLASTN (http://www.ncbi.nlm.nih.gov/blast) at the NIH. The sequences of $T$. s. elegans genes were submitted to GenBank with the following accession numbers: NFKB1: FJ834440, p65: FJ834439, Fth1: FJ834441; MnSOD: FJ834442; Cu/ZnSOD FJ834443; Bcl-xL: FJ834441; Bcl-2: FJ834442.

\subsubsection{Preparation of tissue extracts and western blotting}

Tissue extraction and western blotting were performed as described in chapter 2 . Briefly, aliquots of control and anoxic samples containing $30 \mu \mathrm{g}$ each were loaded onto $10 \%$ polyacrylamide gels. Gels were run at a constant voltage $(180 \mathrm{~V})$ for $45 \mathrm{~min}$ at RT. 
The resolved proteins were transferred onto PVDF membranes at $70 \mathrm{~V}$ for $90 \mathrm{~min}$ at $4^{\circ} \mathrm{C}$.

Blots were blocked for $15 \mathrm{~min}$ in Tris-buffered saline containing $0.05 \%$ Tween-20 (TBST) with $2.5 \%$ milk. The blots were then probed overnight with primary antibodies for P-IкB $\alpha$ (Ser32), p50, or p65 (all from Cell Signaling, diluted 1:1000 in TBST). After incubation, blots were washed several times with TBST and then incubated at RT for 1.5 h with HRP-linked goat anti-rabbit IgG (Cell Signaling, diluted 1:2000 in TBST). Immunoblots were developed using enhanced chemiluminescence (ECL) reagents.

\subsubsection{Preparation of nuclear extracts}

Nuclear extracts were prepared as described in Chapter 2.

\subsubsection{DNA binding assay}

Nuclear extract aliquots containing equal amounts of protein from each sample $(16 \mu \mathrm{g} /$ well) were used to assess the amount of binding by NF- $\mathrm{KB}$ to its response element using a DNA-binding assay from Active Motif. Binding assays were performed according to the manufacturer's protocol. Briefly, NF- $\mathrm{KB}$ binding was carried out for $1 \mathrm{~h}$ at RT with mild agitation on a rocking platform. The plates were then washed with wash buffer to remove unbound transcription factors. NF- $\kappa \mathrm{B}$ p65-specific primary antibody diluted 1:1000 v/v in antibody buffer was then added to each well and incubated for $1 \mathrm{~h}$ at RT without agitation. The wells were then washed three times with washing buffer and the secondary antibody (HRP-linked anti-rabbit antibody diluted 1:1000 v/v in antibody 
buffer) was added to each well and incubated for $1 \mathrm{~h}$ at RT without agitation. The wells were washed five times with washing buffer and the manufacturer's developing solution was then added. After developing for $10 \mathrm{~min}$ in the dark, the stop solution was added and color development was quantified by absorbance readings at $450 \mathrm{~nm}$ with a reference wavelength of $655 \mathrm{~nm}$ as described in chapter 2 .

\subsubsection{Data and statistics}

Data analysis was performed as described in Chapter 2. Significant differences between the groups were determined using either a student's t-test (for the control versus $5 \mathrm{~h}$ of anoxia data) or ANOVA followed by the Student-Newman-Keuls test for the control versus 5 and $20 \mathrm{~h}$ anoxia data. Statistical difference was accepted if $\mathrm{P}<0.05$.

\subsection{RESULTS}

\subsubsection{Expression of phosphorylated I $\mathrm{K} B \alpha$}

Activation of the NF- $\mathrm{KB}$ pathway is mediated by increased phosphorylation of the $\mathrm{I} \kappa \mathrm{B} \alpha$ protein that causes $\mathrm{I} \kappa \mathrm{B} \alpha$ to dissociate from NF- $\mathrm{KB}$. The relative amount of phosphorylated IkBọ(Ser 32) was assessed in the heart, kidney, liver and muscle of T.s. elegans under aerobic conditions and $5 \mathrm{~h}$ of anoxia (FIG.3.2). The polyclonal 
mammalian antibody cross-reacted with a band of $\sim 40 \mathrm{kDa}$ that corresponded with the known molecular size of $\mathrm{I} \kappa \mathrm{B} \alpha$ in other animals. FIG.3.2A shows representative western blots, whereas FIG.3.2B shows a graphical representation of the relative levels of P$\mathrm{I} \kappa \mathrm{B} \alpha$ in normoxic and anoxic turtles. Liver was the only tissue that showed a significant change, with $\mathrm{P}-\mathrm{I} \kappa \mathrm{B} \alpha$ levels rising by $2.0 \pm 0.1$ fold as compared with controls in response to $5 \mathrm{~h}$ of anoxia $(\mathrm{P}<0.05)$. P-IKB $\alpha$ levels remained elevated $(1.8 \pm 0.2$ fold higher than control) in response to $20 \mathrm{~h}$ of anoxia $(\mathrm{P}<0.05$; data not shown).

\subsubsection{Transcript expression of $N F-\kappa B$ subunits in response to anoxia}

Relative transcript levels of the two subunits that make up the NF- $\mathrm{KB}$ dimer, $\mathrm{p} 50$ (NFKB1) and p65, were assessed via reverse-transcriptase PCR in livers of aerobic, $5 \mathrm{~h}$ anoxic, and $20 \mathrm{~h}$ anoxic turtles. A 391 bp sequence was obtained for NFKB1 (FIG.3.3; GenBank ID: FJ834440) and a 268 bp sequence was obtained for $p 65$ (FIG.3.4; GenBank ID: FJ834439). FIG.3.5A shows representative RT-PCR bands of NFKB1 and p65 amplicons with corresponding $\alpha$-tubulin bands under normoxic and anoxic conditions whereas FIG.3.5B shows a graphical representation of changes in the relative levels of these transcripts in response to anoxia. Transcript levels of $N F K B 1$ were significantly increased in response to $5 \mathrm{~h}$ of anoxia (by $2.3 \pm 0.3$ fold compared with controls, $\mathrm{P}<0.05$ ). However, by $20 \mathrm{~h}$ of anoxia exposure, transcript levels of $N F K B 1$ returned to normoxic levels. Levels of $p 65$ transcript showed no significant change in response to anoxia. 


\subsubsection{Protein expression of $N F-\kappa B$ subunits in response to anoxia}

Relative protein levels of the two subunits that make up the NF-KB dimer, p50 and p65, were assessed via western blotting in liver from aerobic, $5 \mathrm{~h}$ anoxic, and $20 \mathrm{~h}$ anoxic turtles (FIG.3.6). The polyclonal mammalian antibodies for p50 and p65 crossreacted with single bands of $\sim 50 \mathrm{kDa}$ (for $\mathrm{p} 50$ ) and $65 \mathrm{kDa}$ (for $\mathrm{p} 65$ ), corresponding to the known molecular weights of these proteins. FIG.3.6B shows a graphical representation of changes in the levels of these proteins in response to anoxia. Levels of p50 were increased significantly in response to $5 \mathrm{~h}$ of anoxia (to $2.9 \pm 0.3$ fold of aerobic value, $\mathrm{P}<0.05$ ) and returned to normoxic values by $20 \mathrm{~h}$ of anoxia. Protein levels of p65 also increased in response to anoxia, rising to $2.2 \pm 0.4$ fold of control values $(\mathrm{P}<0.05)$ and again returning to normoxic control values after $20 \mathrm{~h}$.

\subsubsection{Nuclear levels of $N F-\kappa B$ subunits and DNA binding activity}

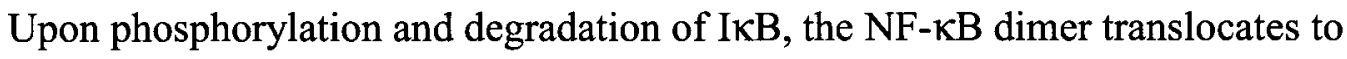
the nucleus and stimulates the transcription of downstream genes. Changes in the nuclear levels of both NF-kB subunits were assessed in T. s. elegans liver in response to 5 and 20 $\mathrm{h}$ of anoxia to determine whether nuclear translocation occurred in response to anoxia (FIG.3.7). Both NF- $\kappa B$ subunits displayed an increase in the nuclear fraction in response to $5 \mathrm{~h}$ of anoxia, with p50 increasing by $1.7 \pm 0.1$ fold $(\mathrm{P}<0.05)$ and $\mathrm{p} 65$ showing a strong increase of $7.3 \pm 0.4$ fold $(\mathrm{P}<0.05)$. By $20 \mathrm{~h}$ of anoxia, $\mathrm{p} 50$ had returned to normoxic levels, and p65 decreased compared to $5 \mathrm{~h}$, but still remained significantly 
higher $(4.8 \pm 0.4$ fold $)$ of control $(\mathrm{P}<0.05)$. These results demonstrate an enhanced presence of NF- $\mathrm{kB}$ subunits in the nucleus in response to anoxia.

The DNA-binding activity of NF- $\mathrm{KB}$ in liver from aerobic, $5 \mathrm{~h}$ anoxic, and $20 \mathrm{~h}$ anoxic turtles was assessed using a transcription factor ELISA (FIG.3.8). A significant increase in DNA binding activity was evident in response to both 5 and $20 \mathrm{~h}$ of anoxia (levels were $1.9 \pm 0.15$ and $1.8 \pm 0.1$ fold higher than controls, respectively; $\mathrm{P}<0.05$ )

\subsubsection{Expression of $N F-\kappa B$ target genes}

Reverse transcriptase (RT) PCR was used to determine whether activation of the $\mathrm{NF}-\mathrm{kB}$ pathway in response to anoxia resulted in enhanced expression of some of its target genes. I examined the expression of target genes involved in antioxidant defense and cell survival. The antioxidant defense genes examined were ferritin heavy chain (Fth1), MnSOD and Cu/ZnSOD. A 367 bp sequence was obtained for Fth1 (FIG.3.9; GenBank ID: FJ834441), a 399 bp sequence for MnSOD (FIG.3.10; GenBank ID: FJ834442), and a 228 bp sequence for $C u / Z n S O D$ (FIG.311; GenBank ID: FJ834443). FIG.3.12A shows representative RT-PCR bands of ferritin heavy chain (Fth1), MnSOD and $C u / Z n S O D$ amplicons with corresponding $\alpha$-tubulin bands under aerobic and anoxic conditions whereas FIG.3.12B shows a graphical representation of the transcript levels of these proteins under aerobic and anoxic conditions. Transcript levels of Fth1 increased to $1.6 \pm 0.04$ fold higher than the control value in response to $5 \mathrm{~h}$ anoxia $(\mathrm{P}<0.05)$ and remained elevated at $1.4 \pm$ fold of control after $20 \mathrm{~h}$ of anoxia $(\mathrm{P}<0.05)$. Transcript levels of $M n S O D$ also increased under anoxia, rising to $2.5 \pm 0.2$ fold of control values ( $\mathrm{P}$ 
$<0.05$ ) in response to $5 \mathrm{~h}$ of anoxia. At $20 \mathrm{~h}$ of anoxia, $M n S O D$ transcript levels were lower than at $5 \mathrm{~h}$ of anoxia, but remained higher than control (at $1.5 \pm 0.1$ fold; $\mathrm{P}<0.05$ ). Levels of $C u / Z n S O D$ increased to $1.7 \pm 0.1$ fold of control $(\mathrm{P}<0.05)$ in response to $5 \mathrm{~h}$ of anoxia and remained elevated at $1.5 \pm 0.1$ fold of control at $20 \mathrm{~h}$ of anoxia $(\mathrm{P}<0.05)$.

The pro-survival genes examined were $B c l-x L$ and $B c l-2$. A 254 bp sequence was obtained for $B c l-x L$ (Genbank ID: FJ83444) and a 237 bp sequence was obtained for $B c l$ 2 (FIG.3.13; GenBank ID: FJ834442). FIG.3.14A shows representative RT-PCR bands of $B c l-2$ and $B c l-x L$ amplicons with corresponding $\alpha$-tubulin bands under aerobic and anoxic conditions whereas FIG.3.14B shows a graphical representation of the transcript levels of these proteins under aerobic and anoxic conditions. Transcript levels of $B c l-x L$ increased to $2.8 \pm 0.4$ fold higher than control levels in response to $5 \mathrm{~h}$ of anoxia $(\mathrm{P}<$ $0.05)$, and remained elevated at $2.5 \pm 0.1$ fold of control $(\mathrm{P}<0.05)$ at $20 \mathrm{~h}$ of anoxia. Similarly, levels of $\mathrm{Bcl}-2$ increased to $2.4 \pm 0.2$ fold of control in response to $5 \mathrm{~h}$ of anoxia $(\mathrm{P}<0.05)$, and remained elevated at $2.4 \pm 0.3$ at $20 \mathrm{~h}$ of anoxia $(\mathrm{P}<0.05)$.

\subsection{DISCUSSION}

The cellular stress response, a cellular defense reaction to environmental stress, is of particular interest to the field of anoxia tolerance because of its role in the protection of cellular macromolecules against stress-induced damage. To better understand the role of the stress response in anoxia tolerance, the present study investigated the activation of the NF-אB pathway in response to anoxic submergence in the red-eared slider, T. s. elegans. 
While many transcription factors are known to be involved in the stress response, NF- $\mathrm{BB}$ is exceptionally important due to the rapidity of its activation and the large number of signals and pathways it controls. Because of the role of $\mathrm{NF}-\mathrm{\kappa B}$ as a central mediator of the stress response, its activation could be key to alleviation of any stress the animal might experience under the anoxic conditions.

Although originally characterized as a central mediator of the immune response, $\mathrm{NF}-\mathrm{\kappa B}$ was later found to be involved in many cellular processes, making it the subject of many studies in different animal models. In mammals, activation of this pathway has been demonstrated in response to low oxygen conditions such as hypoxia and ischemiareperfusion (Cummins and Taylor, 2005; Luedde and Trautwein, 2006; Nichols, 2004; Ridder and Schwaninger, 2009). The nature of this response in ischemia remains somewhat unclear, with some studies demonstrating beneficial effects for NF- $\mathrm{kB}$ (Kuboki et al., 2007; Misra et al., 2003), while others report the benefits of its attenuation (Fan et al., 2004; Luedde et al., 2005; Matsui et al., 2005). The role of NF$\mathrm{\kappa B}$ in anoxia-tolerant models, however, has scarcely been investigated. The present study analyzed the response of NF- $\mathrm{KB}$, as well as some of its target downstream genes, to anoxia exposure in the red-eared slider turtle. The results show that this pathway is activated in the turtle liver in response to anoxia, suggesting a potentially beneficial role for NF- $\mathrm{KB}$ signaling. The present study is the first study to thoroughly examine the response of the NF- $\mathrm{KB}$ pathway to oxygen lack in an anoxia-tolerant animal. 
$N F-\kappa B$ is activated in response to anoxia in turtle liver

Various stimuli induce the phosphorylation of the NF- $\kappa B$ inhibitor, $\mathrm{I} \kappa \mathrm{B}$, resulting in its ubiquitination and degradation. Dissociation of I $\mathrm{KB}$ unmasks the nuclear localization signals on NF- $\kappa B$, allowing it to translocate to the nucleus and induce transcription of target genes (Karin, 1999). Because of the importance of IKB phosphorylation to the activation of the NF- $\mathrm{KB}$ pathway, I initially examined the changes in protein levels of phosphorylated IKB in response to $5 \mathrm{~h}$ of anoxia in four turtle tissues: heart, kidney, liver, and white skeletal muscle (FIG.3.2). The results showed elevated

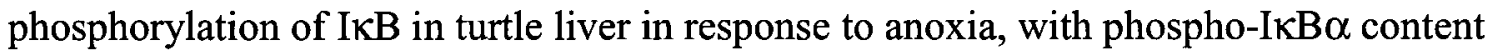
increasing by 2 - fold over control values in response to $5 \mathrm{~h}$ of anoxia exposure. These results suggested the potential for a rapid activation of the NF- $\kappa B$ pathway in response to anoxia in the liver. As such, the liver was chosen for further studies of this pathway.

$\mathrm{NF}-\kappa \mathrm{B}$ is a dimeric transcription factor composed of homo- or hetero-dimers of different members of the Rel family of proteins, with the best studied dimer, and the one most frequently termed as 'NF- $\mathrm{KB}$ ' occurring between p65 (RelA) and p50. Western blotting and RT-PCR studies suggested that both p50 and p65 were up-regulated in response to anoxia. Transcript levels of p50 rose by 2.3 -fold in response to $5 \mathrm{~h}$ of anoxia (FIG3.5), whereas p50 protein levels rose by 2.9-fold (FIG3.6). Interestingly, whereas p65 protein levels showed a significant increase in response to $5 \mathrm{~h}$ of anoxia, rising by 2.2-fold, no change in transcript levels was observed. This suggests that the increase in p65 protein levels might not be due to increased gene expression, but perhaps to enhanced stabilization of the p65 protein. Indeed, some studies have suggested that 
increased stabilization of $\mathrm{p} 65$ is involved in NF- $\mathrm{\kappa B}$ activation (Hamdane et al., 1997; Ryo et al., 2003).

Elevated levels of NF- $\mathrm{KB}$ in isolated liver nuclei were also observed in response to anoxia; p50 rose by 1.7 fold in response to $5 \mathrm{~h}$ of anoxia but dropped back to normoxic levels by $20 \mathrm{~h}$, whereas p65 rose by 7.3 fold after $5 \mathrm{~h}$ anoxia and decreased to 4.8 fold higher than controls by $20 \mathrm{~h}$ (FIG3.7). The enhanced NF-אB presence in the nucleus correlated with the increased binding activity of NF- $\kappa B$ to its DNA element; binding was increased by 1.8-1.9 fold under anoxic conditions (FIG3.8). Overall, these molecular changes suggest a clear activation of the NF- $\kappa B$ pathway in turtle liver in response to anoxia. These results suggest that, at least in the liver, activation of the NF- $\kappa B$ pathway in response to oxygen limitation could be beneficial to the animal.

$N F-\kappa B$ target genes are upregulated in the turtle liver in response to anoxia

Activation of NF- $\mathrm{kB}$ typically leads to up-regulation of target genes. Of particular interest to oxygen limitation are those targets that are involved in antioxidant defense and cell survival. Enhancement of antioxidant defenses in response to oxygen limitation can have several benefits. As previously mentioned, the electron carriers of the mitochondrial respiratory chain become reduced under low-oxygen conditions and reintroduction of oxygen causes their immediate reoxidation, often leading to a transient overproduction of reactive oxygen species (Ruuge et al., 1991). This can overwhelm the existing cellular antioxidant defenses and cause damage to cellular macromolecules (Cordis et al., 1995, 1998). In mammalian systems undergoing ischemia and reperfusion, these events can 
lead to severe damage and organ failure (Bolli and Marban, 1999; Lipton, 1999). Therefore, enhancement of the cellular antioxidant machinery during oxygen limitation could serve as a preparatory mechanism for eventual recovery and minimize any oxidative damage (Hermes-Lima et al., 2001). Some oxidative damage could also occur early on during the hypoxic transition episode. In this case the reduction of the mitochondrial electron carriers in response to low oxygen could lead to excessive formation of reactive oxygen species from the limited oxygen that is still present in the cell, creating oxidative stress. Indeed, previous studies in mammalian cells have demonstrated that hypoxia-mediated partial inhibition of mitochondrial electron transport can result in redox changes in the electron carriers, with consequent enhanced generation of reactive oxygen species (Dawson et al., 1993). Enhanced antioxidant defenses could be beneficial here as they would alleviate any stress inflicted on the cell as a result of ROS production during the hypoxic transition phase. It should also be noted that NF- $\mathrm{KB}$ is known to be activated by oxidative stress (Rupec and Baeurle, 1995; Schreck et al., 1991), so any ROS formed during the hypoxic transition period might actually be an inducer for NF- $\mathrm{\kappa B}$ activation.

To confirm an action of NF- $\mathrm{KB}$ activation under anoxic conditions, I assessed transcript levels of several known NF- $\mathrm{KB}$ target genes that function in antioxidant defense. These included the heavy chain of ferritin (Kwak et al, 1995), copper/zinc superoxide dismutase (Cu/Zn SOD) (Rojo et al., 2004) and manganese superoxide dismutase (MnSOD) (Das et al., 1995). Ferritin reduces oxidative stress by sequestering redox active iron inside cells, and by doing so, reducing the potential for hydroxyl radical formation via the Fenton reaction (Hentze et al., 2004). $\mathrm{Cu} / \mathrm{Zn}$ - and $\mathrm{MnSOD}$ reduce 
oxidative stress by catalyzing the dismutation of superoxide radicals into water and hydrogen peroxide. Studies of ischemia in mammalian models suggested beneficial roles for ferritin (Berberat et al., 2003; Berenshtein et al., 2002; Obolensky et al., 2008; Tacchini et al., 1997), as well as SOD (Beresewicz et al., 1998; Scorziello et al., 2007; Yamashita et al., 1994). Transcript levels of all three of these proteins rose significantly in response to anoxia in turtle liver (FIG3.12): ferritin $H$ increased 1.4-1.6-fold, $M n S O D$ rose 1.5-2.5 fold and $\mathrm{Cu} / \mathrm{Zn} S O D$ increased 1.5-1.7-fold. Up-regulation of these genes in response to anoxia further supports the idea of a preparatory mechanism against oxidative stress.

Pro-survival proteins are also important to long term anoxia survival. There is growing evidence that induction of apoptosis is oxygen-dependent, and several studies reported increased apoptosis in response to oxygen-limitation in oxygen sensitive cells (reviewed by Brunelle and Chandel, 2002). Tissues of the red-eared slider, however, seem to recover from anoxia without any apparent damage, suggesting that inhibition of apoptosis might occur on the molecular level. The promotion or inhibition of apoptosis is controlled by two conserved pathways, the death receptor pathway and the mitochondrial pathway, and the decision to initiate apoptosis often depends on the levels of pro- or antiapoptotic proteins (Gupta, 2001). The NF-אB pathway is known to control several genes involved in the regulation of apoptosis, including the anti-apoptotic genes Bcl-2 (Catz and Johnson, 2001; Zong et al., 1999) and Bcl-xL (Chen et al., 1999; Lee et al., 1999). These proteins reside in the outer mitochondrial membrane and stop apoptosis by preventing the loss of outer mitochondrial membrane integrity (Janumyan et al., 2003; Kluck et al., 1997). Studies in mammalian models show that enhanced expression of 
these proteins could limit apoptosis in cells (McClintock et al., 2002; Piret et al., 2005). In addition, some studies have shown that Bcl-2 family members are expressed and have a protective role during mammalian ischemic preconditioning (Barrier et al., 2005; Hu and Lu, 2005; Peralta et al., 2002). I examined the expression of $B c l-x L$ and $B c l-2$ transcripts in turtle liver in response to anoxia. Both genes were up-regulated at the transcript level in the turtle liver in response to anoxia (FIG3.14). $B c l-x L$ was upregulated by 2.8 -fold in response to $5 \mathrm{~h}$ of anoxia and remained high at 2.5 -fold at $20 \mathrm{~h}$. Similarly, $B c l-2$ was induced by 2.4 -fold during $5 \mathrm{~h}$ of anoxia, and remained elevated at 2.4- fold of control at $20 \mathrm{~h}$ of anoxia. Up-regulation of these proteins might be involved in promoting cell survival in response to anoxia by counteracting any pro-apoptotic signals that might occur due to the stress of oxygen limitation.

In summary, the present study provides the first demonstration of activation of the $\mathrm{NF}-\mathrm{kB}$ pathway, as well as several downstream genes, in response to anoxia in the liver of an important model species for natural anoxia tolerance (T. s. elegans). These data suggest that activation of NF- $\mathrm{KB}$ signaling may be an integral part of the anoxia survival strategy of this species. 
TABLE 3.1. List of primers used in chapter 3.

\begin{tabular}{|c|c|c|c|}
\hline Gene/primer & Primer sequence (5'-3') & $\begin{array}{c}\text { Annealing } \\
\text { temp. } \\
\left({ }^{\circ} \mathrm{C}\right)\end{array}$ & $\begin{array}{c}\text { Expected } \\
\text { product size } \\
\text { (bp) }\end{array}$ \\
\hline $\begin{array}{l}N F K B 1(p 50) \\
\text { forward }\end{array}$ & GCAAACCTKGGAATACTTCA & \multirow[t]{2}{*}{54} & \multirow[t]{2}{*}{$\sim 500$} \\
\hline $\begin{array}{l}N F K B 1(p 50) \\
\text { reverse }\end{array}$ & GCAAAYTGTCTATGWACATC & & \\
\hline p65 forward & TGGGIATTCAGTGTGTGAAG & \multirow[t]{2}{*}{54} & \multirow[t]{2}{*}{$\sim 300$} \\
\hline p65 reverse & CACAGAGCAGGAAGATCTCA & & \\
\hline Fth1 forward & GCCGTCAACCGCCAGATCAA & \multirow[t]{2}{*}{54} & \multirow[t]{2}{*}{$\sim 400$} \\
\hline Fthl reverse & CAGATTGGTCGCATGGTCA & & \\
\hline$M n S O D$ forward & CACCACAGYAARCACCAYGC & \multirow[t]{2}{*}{54} & \multirow[t]{2}{*}{$\sim 500$} \\
\hline$M n S O D$ reverse & CTCCCAGTTGATMACATTCC & & \\
\hline $\begin{array}{l}\mathrm{Cu} / \mathrm{ZnSOD} \\
\text { forward }\end{array}$ & TGTACCAGYGCAGGTSCTCA & \multirow[t]{2}{*}{54} & \multirow[t]{2}{*}{$\sim 300$} \\
\hline $\begin{array}{l}\mathrm{Cu} / \mathrm{ZnSOD} \\
\text { reverse }\end{array}$ & AATYACWCCACAAGCYAAAC & & \\
\hline$B c l-x L$ forward & GGATACAGCTGGAGTC & \multirow[t]{2}{*}{62.5} & \multirow[t]{2}{*}{$\sim 300$} \\
\hline$B c l-x L$ reverse & ACTACCTGCTCAAAGCTCTG & & \\
\hline$B c l-2$ forward & GARAGCGTCAACAGGGAGATG & \multirow[t]{2}{*}{54} & \multirow[t]{2}{*}{$\sim 300$} \\
\hline$B c l-2$ reverse & TTGGCAGTAAATAGCTGATT & & \\
\hline $\begin{array}{l}\alpha \text {-tubulin } \\
\text { forward }\end{array}$ & AAGGAAGATGCTGCCAATAA & \multirow[t]{2}{*}{54} & \multirow[t]{2}{*}{$\sim 700$} \\
\hline$\alpha$-tubulin reverse & GGTCACATTTCACCATCTG & & \\
\hline
\end{tabular}


FIG.3.1

\section{STIMULATORY SIGNALS}

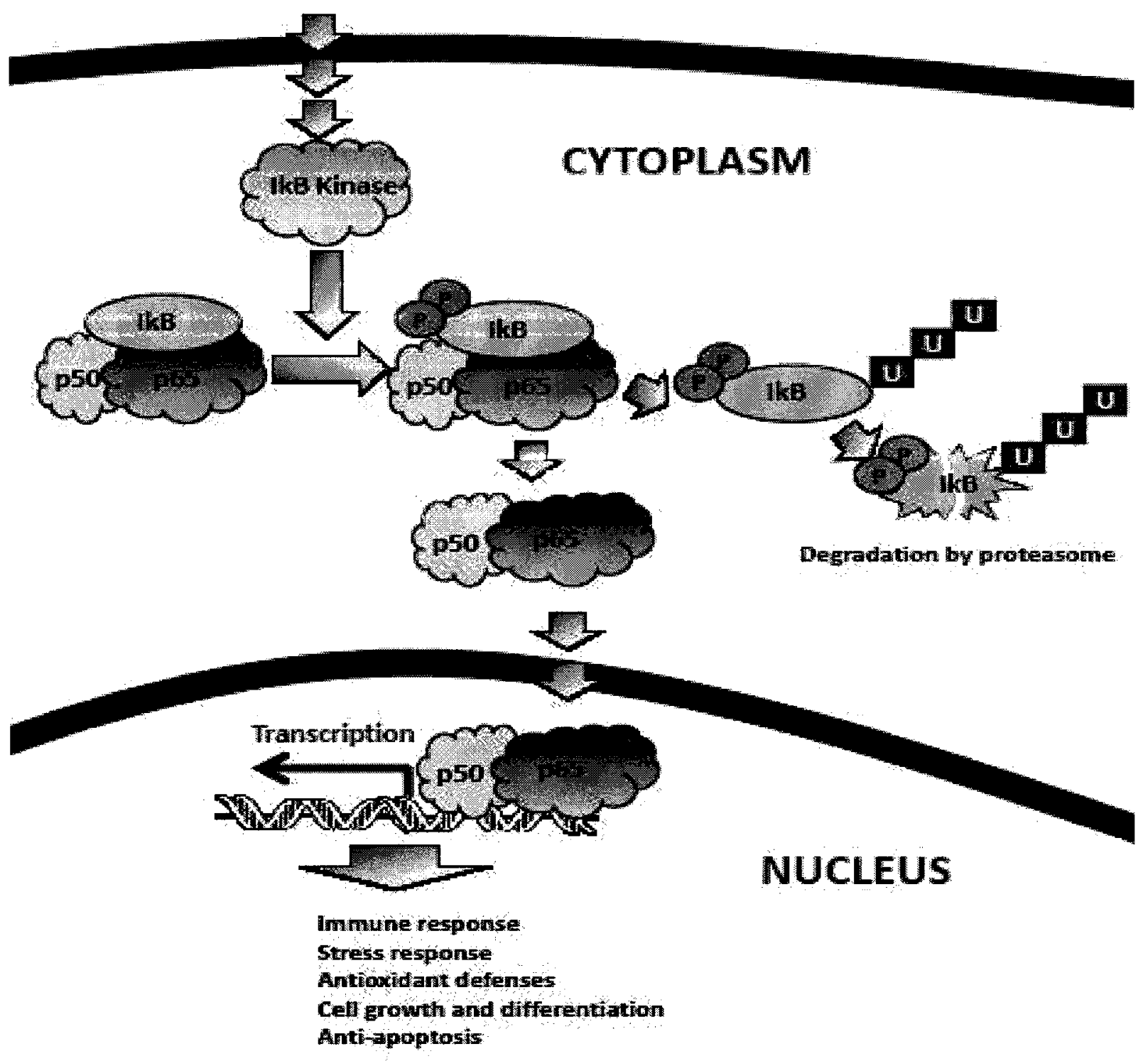

FIG.3.1. Activation of NF- $\mathrm{KB}$ in response to anoxia. Under normal conditions, the NF$\mathrm{\kappa B}$ dimer (composed of the proteins $\mathrm{p} 50$ and p65) is retained in the cytoplasm through interaction with its inhibitor protein, I $\mathrm{kB}$. In response to various stimuli, the IkB kinase becomes activated and phosphorylates $\mathrm{I} \kappa \mathrm{B}$, thereby targeting it for ubiquitination and subsequent degradation by the proteasome. This leaves NF- $\kappa$ B free to move to the nucleus and activate transcription of various target genes. 
FIG.3.2

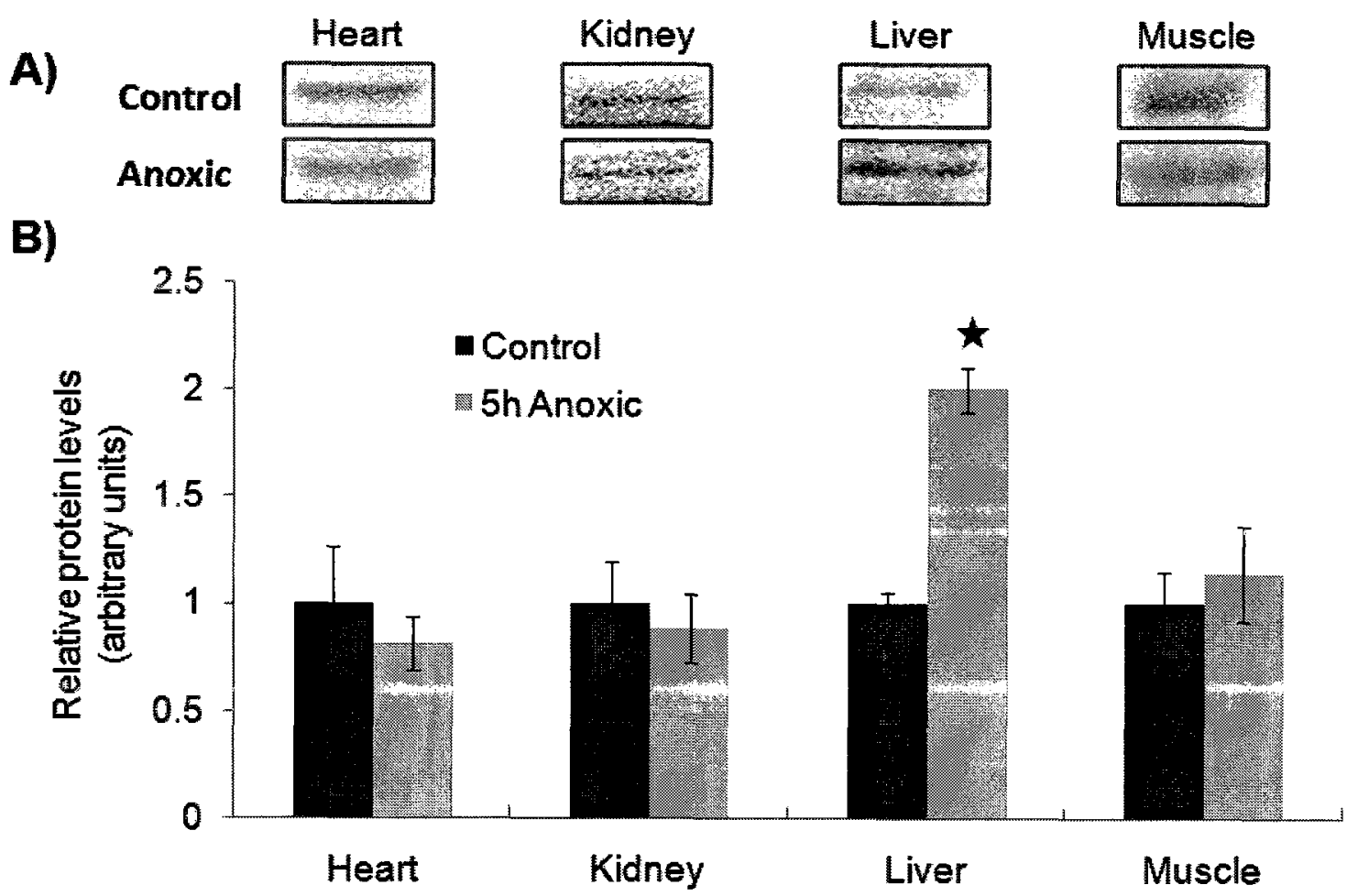

FIG. 3.2. Effect of $5 \mathrm{~h}$ of anoxic submergence on levels of phosphorylated IkB $\alpha$ (Ser32) in the heart, kidney, liver, and white skeletal muscle of T.s. elegans. Histogram shows normalized protein levels; data are means \pm S.E.M, $n=3-5$ independent trials. * Significantly different from the corresponding control $(\mathrm{P}<0.05)$. 
FIG. 3.3

1

1

61

21

121

41

181

61

241

81

301

101

361

121

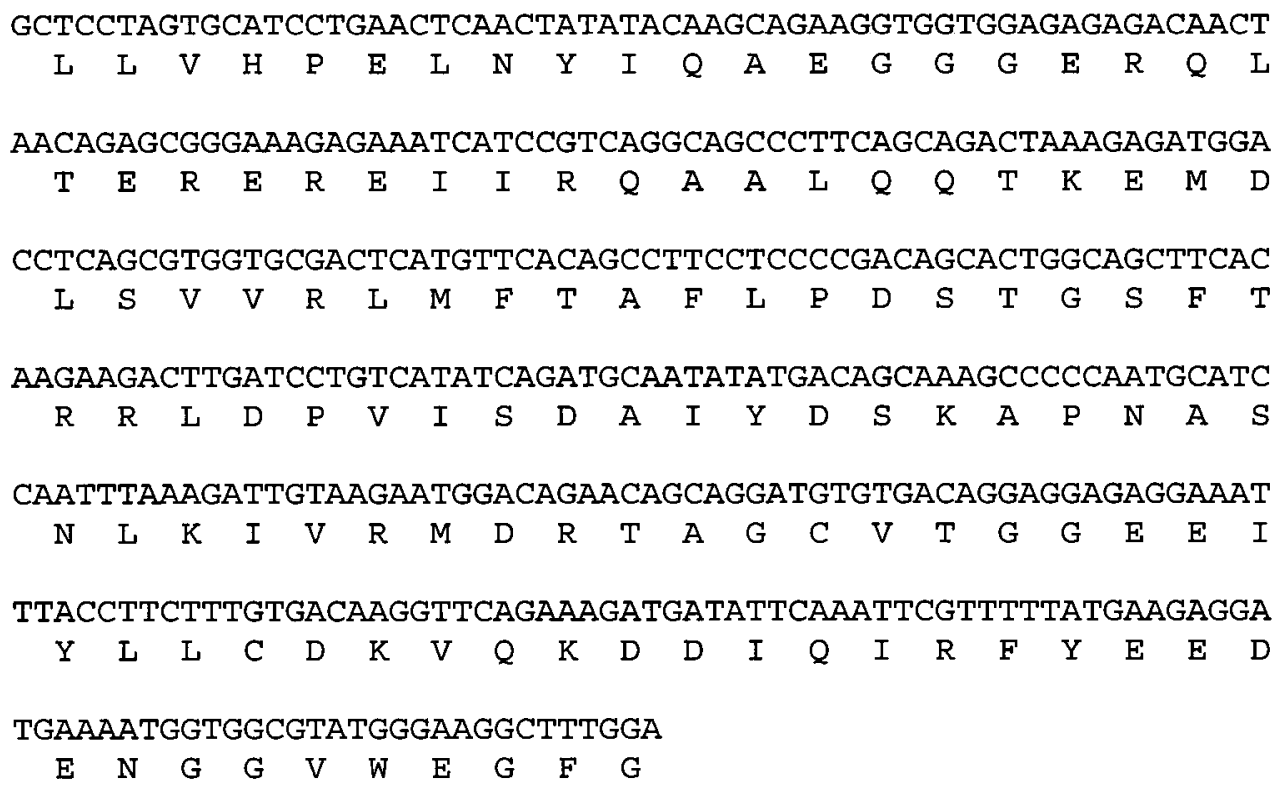

FIG. 3.3. Nucleotide and deduced amino acid sequence for $T$. s. elegans partial $N F K B 1$ sequence. Nucleotides and amino acids are numbered on the left. The nucleotide sequence was 391 nucleotides in length, while the amino acid sequence was 130 amino acids in length. 
FIG. 3.4

1

1

61

21

121

41

181

61

241

81

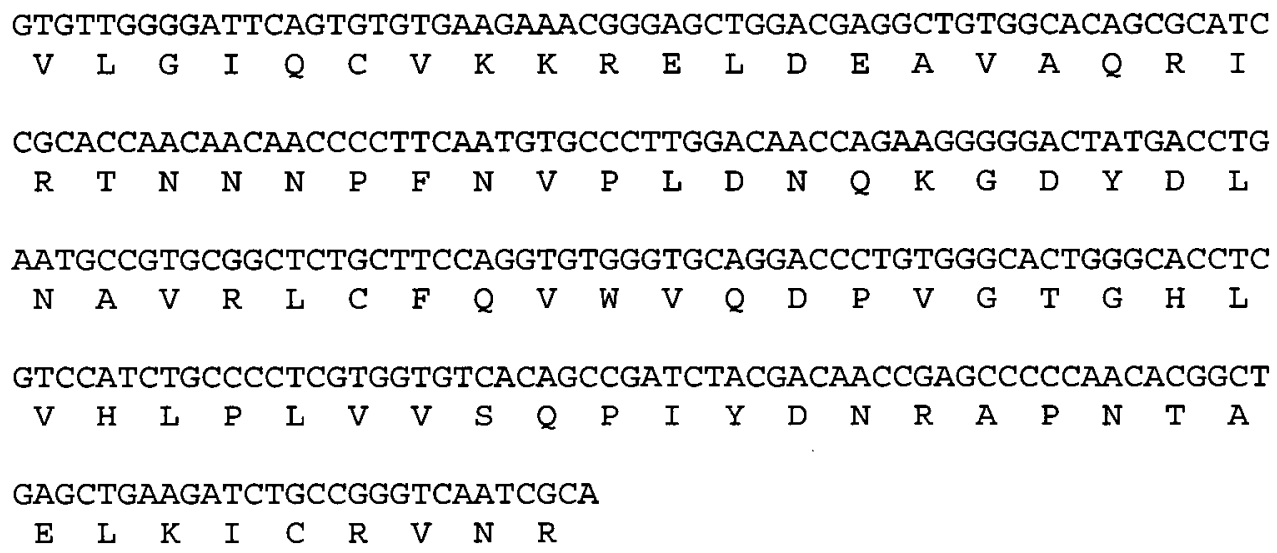

FIG. 3.4. Nucleotide and deduced amino acid sequence for T. $s$. elegans partial $p 65$ sequence. Nucleotides and amino acids are numbered on the left. The nucleotide sequence was 268 nucleotides in length, while the amino acid sequence was 89 amino acids in length. 
FIG. 3.5

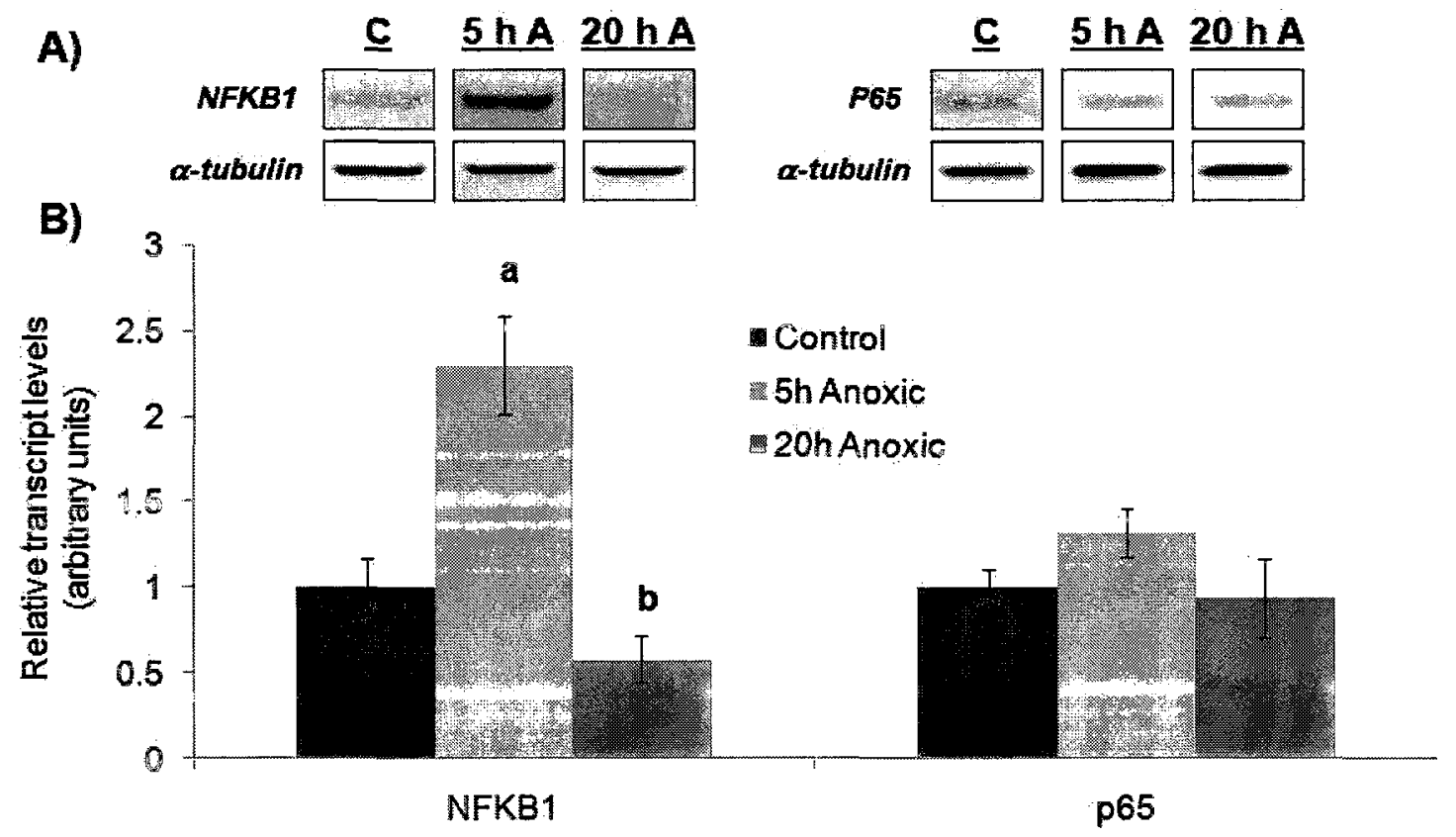

FIG. 3.5.Effect of 5 and $20 \mathrm{~h}$ of anoxic submergence on transcript levels of the proteins making up the NF- $\mathrm{kB}$ dimer, NFKB1 (p50) and $\mathrm{p} 65$ in T. s. elegans liver. (A) Representative RT-PCR bands of $N F K B 1$ and $p 65$ amplicons with corresponding $\alpha$ tubulin bands under normoxic control conditions (C), $5 \mathrm{~h}$ of anoxia (5hA), and $20 \mathrm{~h}$ of anoxia (20hA). (B) Histogram showing normalized transcript levels under control and anoxic conditions; bands were normalized against the corresponding $\alpha$-tubulin bands from the same sample. Data are means \pm S.E.M., $n=3-4$ independent trials. a: Significantly different from the corresponding control $(\mathrm{P}<0.05)$; b: significantly different from the $5 \mathrm{~h}$ anoxic value $(\mathrm{P}<0.05)$. 
FIG. 3.6

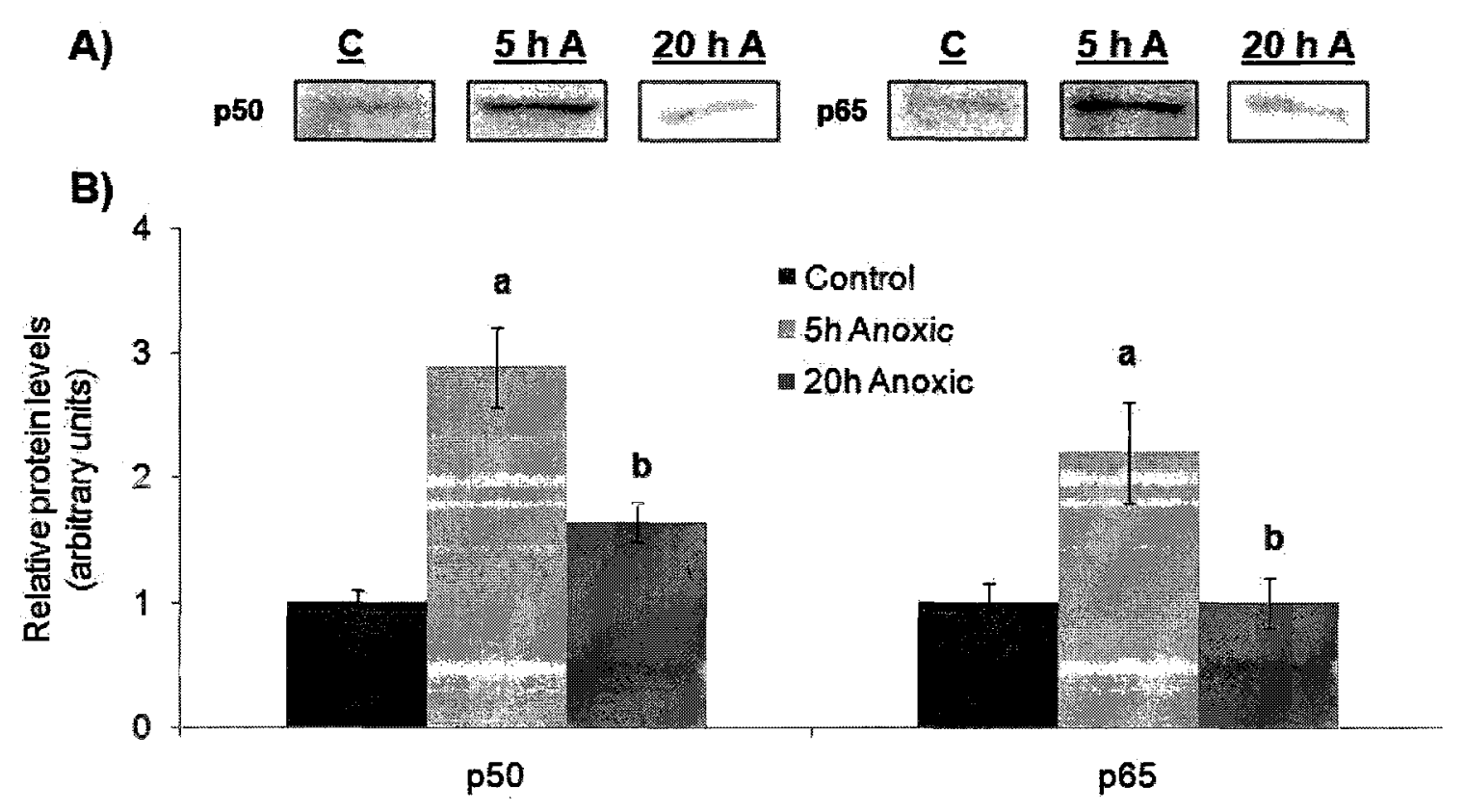

FIG. 3.6. Effect of 5 and $20 \mathrm{~h}$ of anoxic submergence on the protein levels of the proteins making up the NF- $\mathrm{KB}$ dimer, p50 and p65 in T. s. elegans liver. (A) Representative western blot bands under normoxia (C), $5 \mathrm{~h}$ of anoxia (5hA) and $20 \mathrm{~h}$ of anoxia (20hA). (B) Histogram showing normalized protein levels; data are means \pm S.E.M., $n=3-4$ independent trials. a: Significantly different from the corresponding control $(\mathrm{P}<0.05)$; b: significantly different from the $5 \mathrm{~h}$ anoxic value $(\mathrm{P}<0.05)$. 
FIG. 3.7

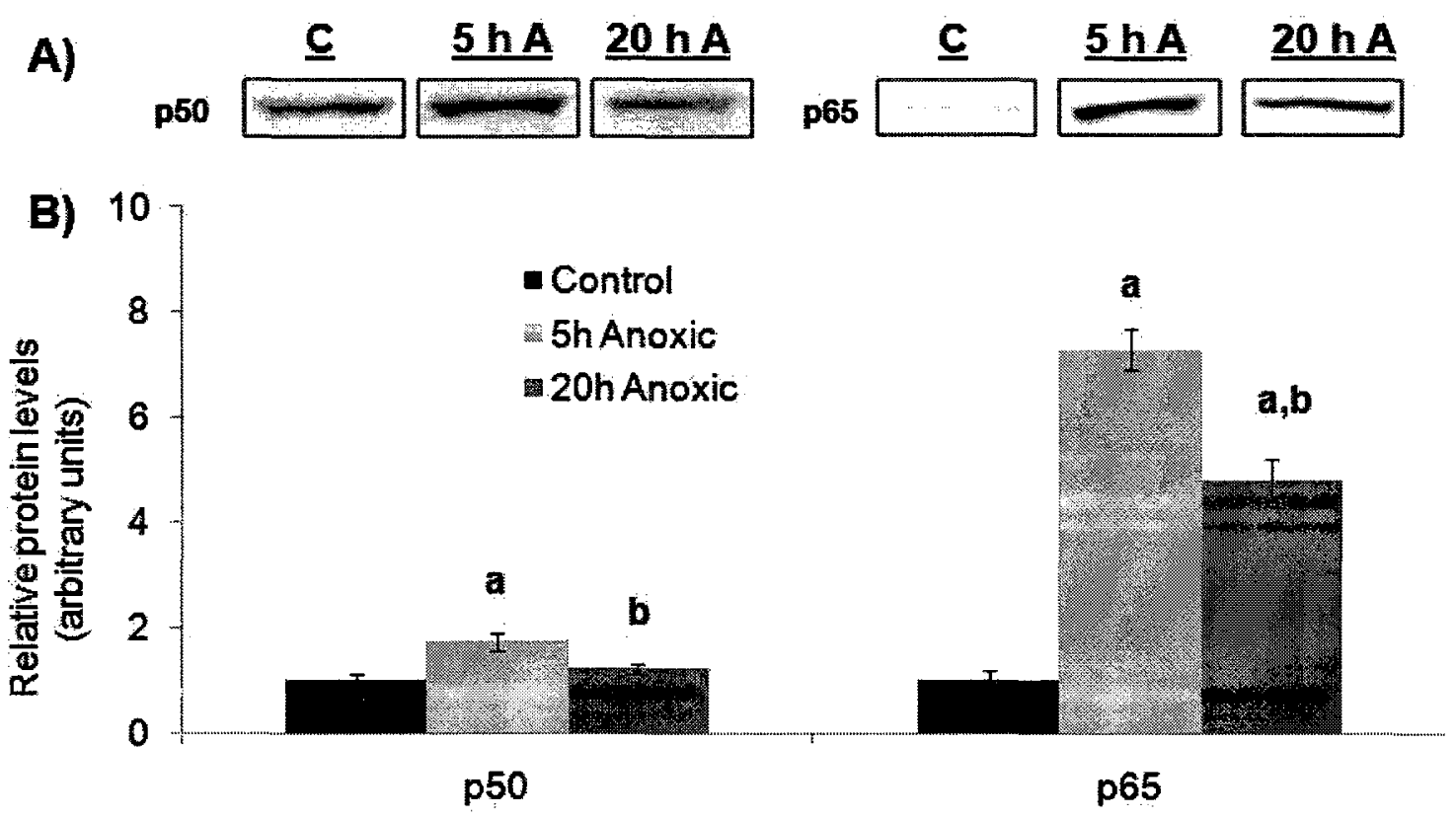

FIG. 3.7. Effect of 5 and $20 \mathrm{~h}$ of anoxic submergence on nuclear protein levels of the proteins making up NF- $\mathrm{kB}$ dimer, p50 and $\mathrm{p} 65$ in T. s. elegans liver. (A) Representative western blot bands under normoxia (C), $5 \mathrm{~h}$ of anoxia (5hA) and $20 \mathrm{~h}$ of anoxia (20hA). (B) Histogram showing normalized protein levels; data are means \pm S.E.M., $n=3-4$ independent trials. a: Significantly different from the corresponding control $(\mathrm{P}<0.05)$; $\mathrm{b}$ : significantly different from the $5 \mathrm{~h}$ anoxic value $(\mathrm{P}<0.05)$. 
FIG. 3.8

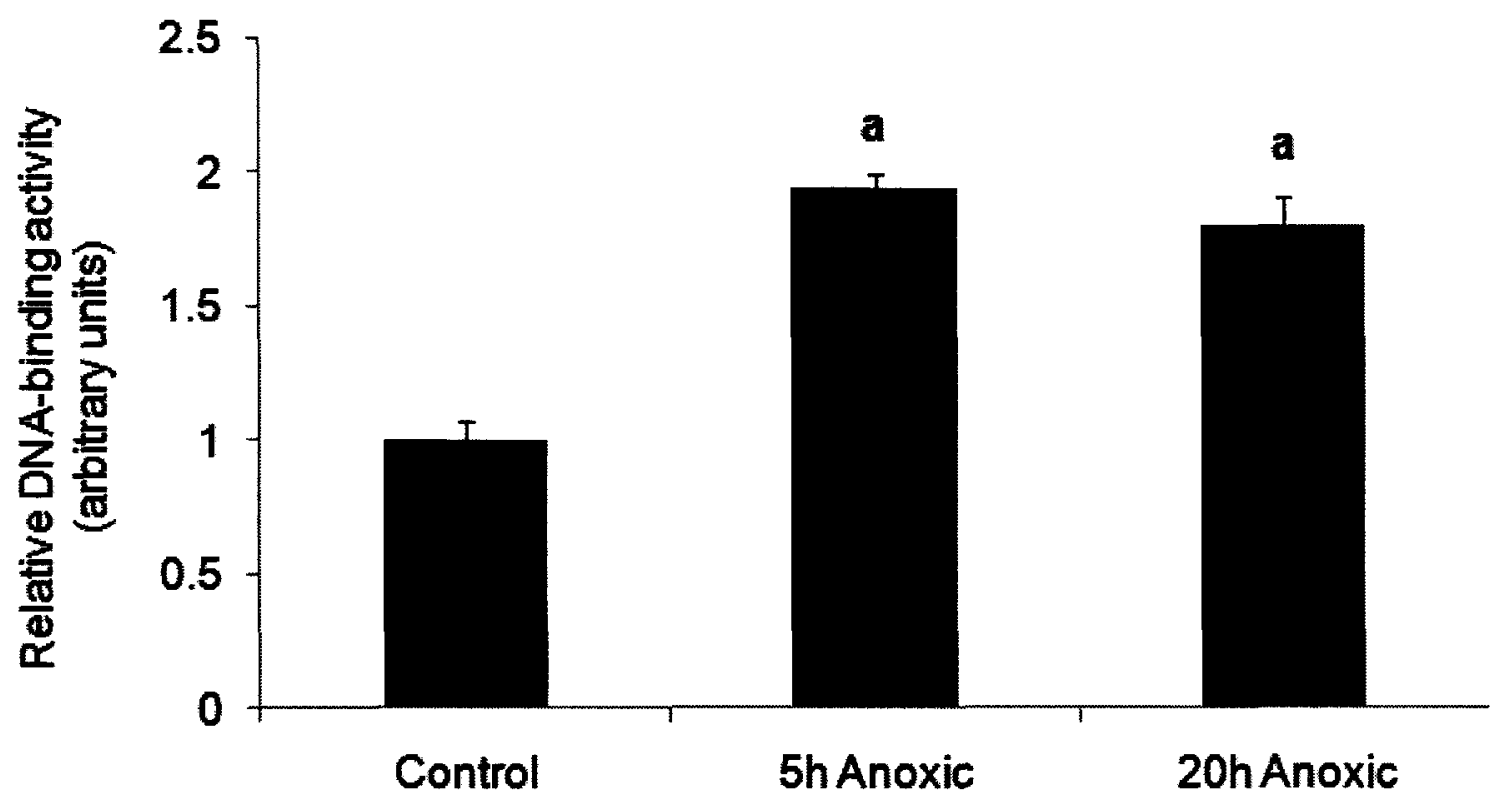

FIG. 3.8. Effect of 5 and $20 \mathrm{~h}$ of anoxic submergence on the DNA-binding activity of NF- $\kappa \mathrm{B}$ in $T$. $s$. elegans liver. Data are means \pm S.E.M., $\mathrm{n}=4$ independent trials. a: Significantly different from the corresponding control, $\mathrm{P}<0.01$. 


\section{FIG. 3.9}

1

1

61

21

121

41

181

61

241

81

301

101

361

121

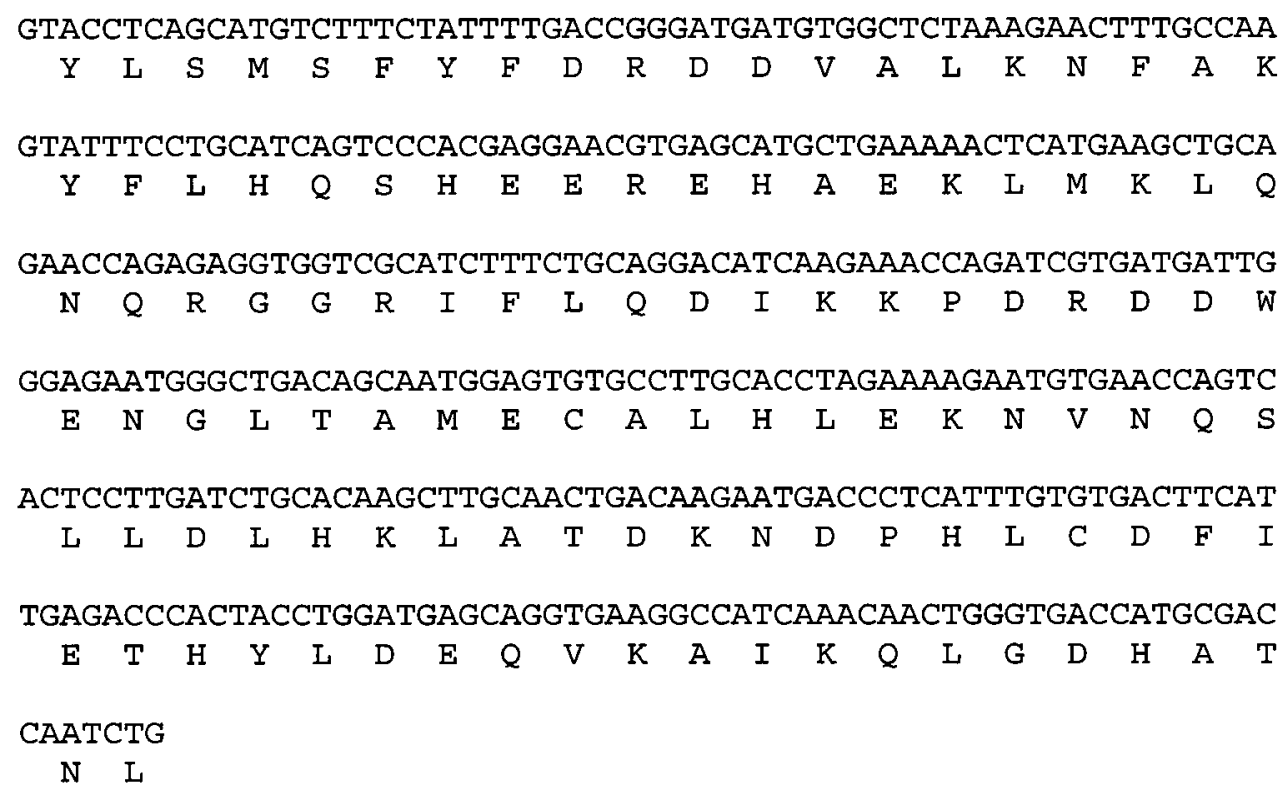

FIG. 3.9. Nucleotide and deduced amino acid sequence for $T$. s. elegans partial Fth1 sequence. Nucleotides and amino acids are numbered on the left. The nucleotide sequence was 367 nucleotides in length, while the amino acid sequence was 122 amino acids in length. 
FIG. 3.10

1

1

61

20

121

40

181

60

241

80

301

100

361

120
CAAAAGGTGATGTTACAGCTCAGGTGTCTCTTCAGCCTGCACTAAAGTTCAATGGTGGGG

$\begin{array}{lllllllllllllllllll}K & G & D & V & T & A & Q & V & S & L & Q & P & A & L & K & F & N & G & G\end{array}$

GTCACATCAACCACACCATCTTCTGGACAAACCTTTCTCCTAACGGGGGAGGAGAGCCTC $\begin{array}{llllllllllllllllllll}G & H & I & N & H & T & I & F & W & T & N & L & S & P & N & G & G & G & E & P\end{array}$

AAGGGGAACTGATGGAAGCCATCAAGCGTGACTTTGGCTCCTTTGGAAACTTCAAGGAGA

$\begin{array}{llllllllllllllllllll}Q & G & E & L & M & E & A & I & K & R & D & F & G & S & F & G & N & F & K & E\end{array}$

AGCTGACGGCAGTATCGGTTGGTGTTCAAGGTTCAGGATGGGGGTGGCTTGGTTTTAACC $\begin{array}{llllllllllllllllllll}K & \mathrm{~L} & \mathrm{~T} & A & \mathrm{~V} & \mathrm{~S} & \mathrm{~V} & \mathrm{G} & \mathrm{V} & \mathrm{Q} & \mathrm{G} & \mathrm{S} & \mathrm{G} & \mathrm{W} & \mathrm{G} & \mathrm{W} & \mathrm{L} & \mathrm{G} & \mathrm{F} & \mathrm{N}\end{array}$ AGGACCAAGGCCGTCTGCAGGTCACTGCTTGTTACAATCAAGACCCTCTGCAAGGAACAA $\begin{array}{llllllllllllllllllll}Q & D & Q & G & R & L & Q & V & T & A & C & Y & N & Q & D & P & L & Q & G & T\end{array}$ CAGGTCTTATTCCTCTGCTAGGAATTGATGTGTGGGAGCATGCTTATTATCTTCAGTATA $\begin{array}{llllllllllllllllllll}T & G & \text { L } & \text { I } & \text { P } & \text { L } & \text { L } & \text { G } & \text { I } & \text { D } & \text { V } & \text { W } & \text { E } & \text { H } & \text { A } & \text { Y } & \text { Y } & \text { L } & \text { Q } & \text { Y }\end{array}$ AAAATGTTAGACCTGACTATCTAAAAGCTATCTGGAATG $\begin{array}{llllllllllllll}K & N & V & R & P & D & Y & L & K & A & I & W & N\end{array}$

FIG. 3.10. Nucleotide and deduced amino acid sequence for T. s. elegans partial $M n S O D$ sequence. Nucleotides and amino acids are numbered on the left. The nucleotide sequence was 399 nucleotides in length, while the amino acid sequence was 133 amino acids in length. 


\section{FIG. 3.11}

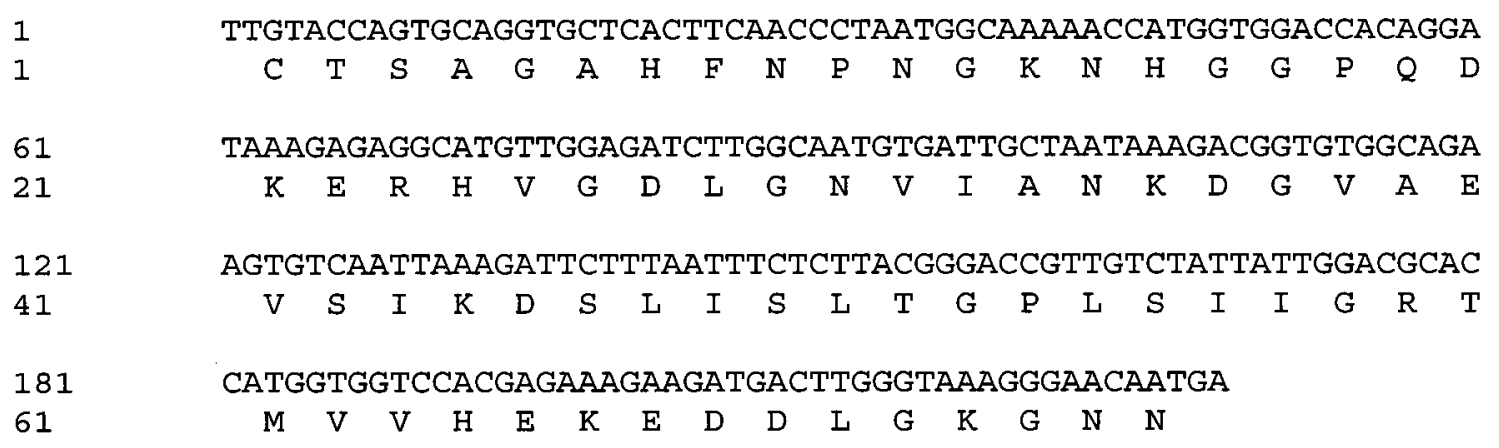

FIG. 3.11. Nucleotide and deduced amino acid sequence for $T$. s. elegans partial $\mathrm{Cu} / \mathrm{ZnSOD}$ sequence. Nucleotides and amino acids are numbered on the left. The nucleotide sequence was 229 nucleotides in length, while the amino acid sequence was 76 amino acids in length. 
FIG. 3.12

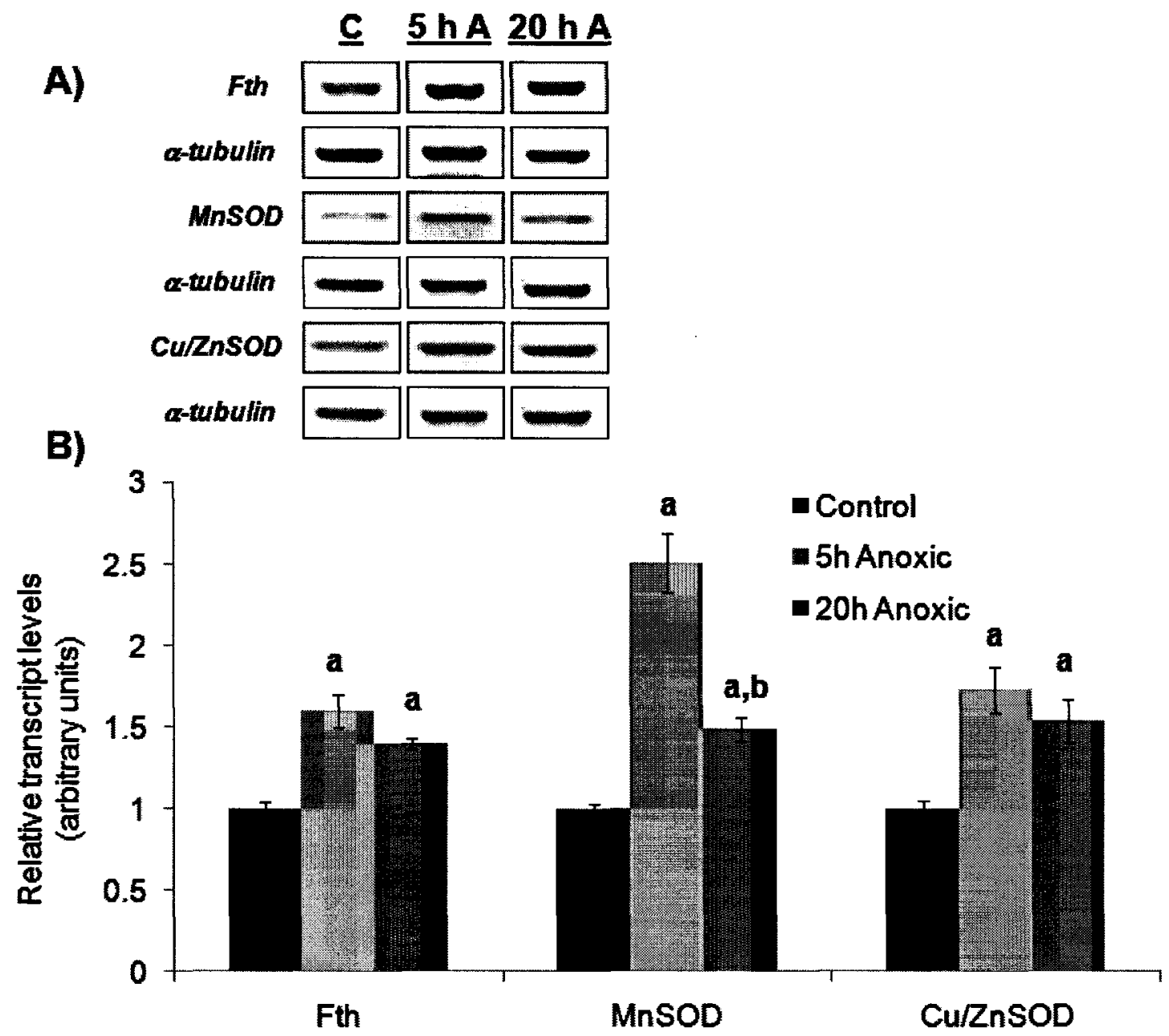

FIG. 3.12. Effect of 5 and $20 \mathrm{~h}$ of anoxic submergence on transcript levels of antioxidant target genes of NF-KB in $T$. s. elegans liver. (A) Representative RT-PCR bands of Ferritin heavy chain (Fth), MnSOD and Cu/ZnSOD amplicons with corresponding $\alpha$ tubulin bands under normoxia (C), $5 \mathrm{~h}$ of anoxia (5hA) and $20 \mathrm{~h}$ of anoxia (20hA). (B) Histogram showing normalized transcript levels under control and anoxic conditions; Bands were normalized against the corresponding $\alpha$-tubulin bands from the same sample. Data are means \pm S.E.M., $n=3-4$ independent trials. a: Significantly different from the corresponding control $(\mathrm{P}<0.05)$; $\mathrm{b}$ : significantly different from the $5 \mathrm{~h}$ anoxic value $(\mathrm{P}<$ $0.05)$. 
FIG. 3.13

1

1

61

20

121

40

181

60
AGAGCGTCAACAGGGAGATGTCGCCTCTTGTGGACAGCATTGCTGTGTGGATGACCGAGT

$\begin{array}{lllllllllllllllllll}\mathrm{S} & \mathrm{V} & \mathrm{N} & \mathrm{R} & \mathrm{E} & \mathrm{M} & \mathrm{S} & \mathrm{P} & \mathrm{L} & \mathrm{V} & \mathrm{D} & \mathrm{S} & \mathrm{I} & \mathrm{A} & \mathrm{V} & \mathrm{W} & \mathrm{M} & \mathrm{T} & \mathrm{E}\end{array}$

ACCTGAACAGACACCTACACAACTGGATCCAAGACAACGGAGGCTGGGATGCCTTTGTGG $\begin{array}{llllllllllllllllllll}\mathrm{Y} & \mathrm{L} & \mathrm{N} & \mathrm{R} & \mathrm{H} & \mathrm{I} & \mathrm{H} & \mathrm{N} & \mathrm{W} & \mathrm{I} & \mathrm{Q} & \mathrm{D} & \mathrm{N} & \mathrm{G} & \mathrm{G} & \mathrm{W} & \mathrm{D} & \mathrm{A} & \mathrm{F} & \mathrm{V}\end{array}$

AGTTGTACGGCAGAAACATGAGGCCTTTGTTTGATTTCTCCTGGATCTCTTTGAAGACTA $\begin{array}{llllllllllllllllllll}E & \mathrm{~L} & \mathrm{Y} & \mathrm{G} & \mathrm{R} & \mathrm{N} & \mathrm{M} & \mathrm{R} & \mathrm{P} & \mathrm{L} & \mathrm{F} & \mathrm{D} & \mathrm{F} & \mathrm{S} & \mathrm{W} & \mathrm{I} & \mathrm{S} & \mathrm{L} & \mathrm{K} & \mathrm{T}\end{array}$

TCCTAAGTCTGGCTCTGGTGGGAGCTTGCATCACCCTTGGCGCTTATCTGGGACATA $\begin{array}{lllllllllllllllllll}I & \mathrm{~L} & \mathrm{~S} & \mathrm{~L} & \mathrm{~A} & \mathrm{~L} & \mathrm{~V} & \mathrm{G} & \mathrm{A} & \mathrm{C} & \mathrm{I} & \mathrm{T} & \mathrm{L} & \mathrm{G} & \mathrm{A} & \mathrm{Y} & \mathrm{L} & \mathrm{G} & \mathrm{H}\end{array}$

FIG. 3.13. Nucleotide and deduced amino acid sequence for $T$. $s$. elegans partial $B c l 2$ sequence. Nucleotides and amino acids are numbered on the left. The nucleotide sequence was 239 nucleotides in length, while the amino acid sequence was 79 amino acids in length. 
FIG. 3.14
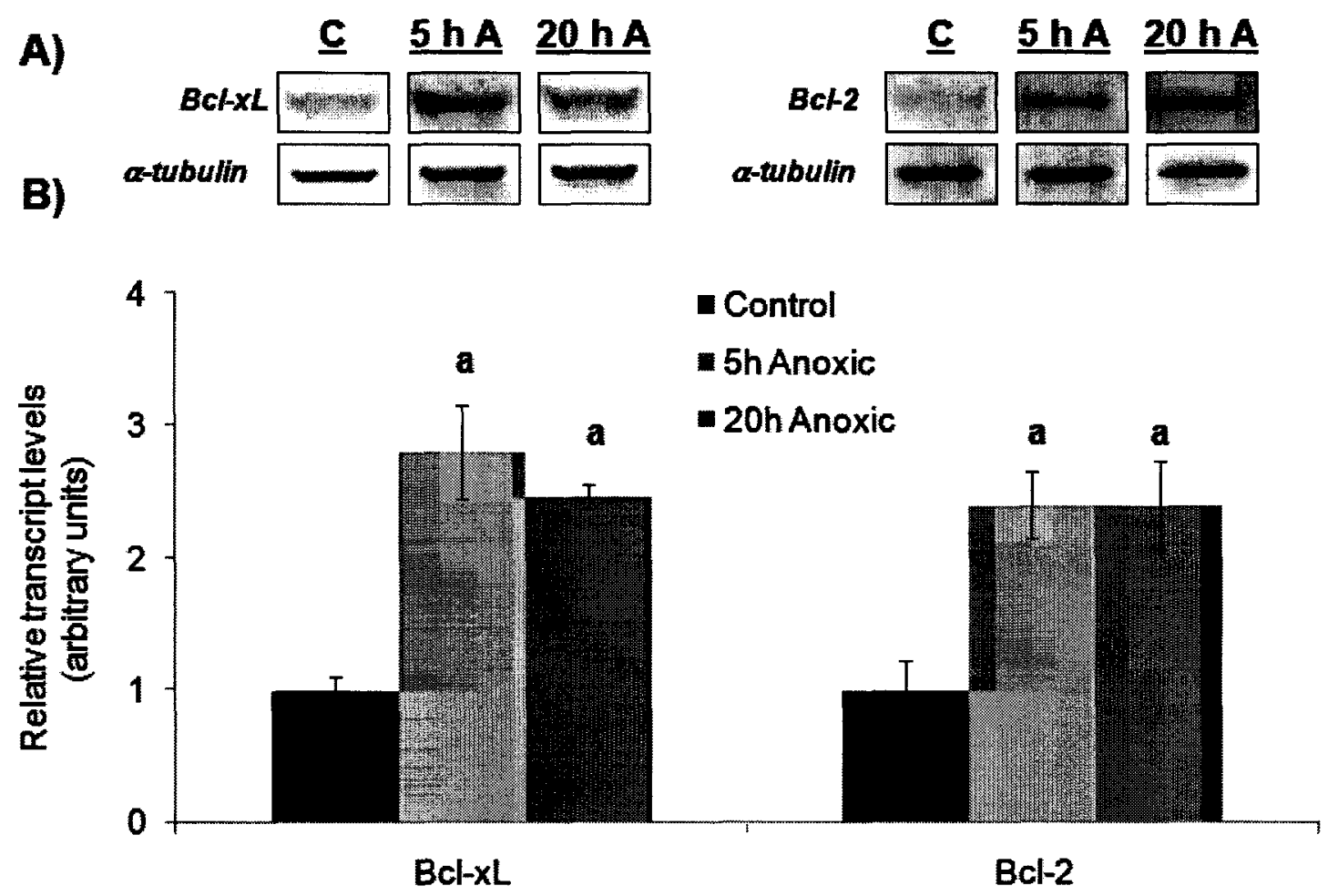

FIG. 3.14. Effect of 5 and $20 \mathrm{~h}$ of anoxic submergence on transcript levels of prosurvival target genes of NF- $\mathrm{KB}$ in T. s. elegans liver. (A) Representative RT-PCR bands of $B c l-x L$ and $B c l-2$ amplicons with corresponding $\alpha$-tubulin bands under normoxia (C), $5 \mathrm{~h}$ of anoxia (5hA) and $20 \mathrm{~h}$ of anoxia (20hA). (B) Histogram showing normalized transcript levels under control and anoxic conditions; Bands were normalized against the corresponding $\alpha$-tubulin bands from the same sample. Data are means \pm S.E.M., $n=3$ independent trials. a: Significantly different from the corresponding control $(\mathrm{P}<0.05)$. 


\section{CHAPTER 4}

\section{Activation of the FoxO transcription factors in response to anoxia in the turtle $T$. s. elegans}

(Publication in preparation) 


\subsection{INTRODUCTION}

The forkhead proteins are a huge family of evolutionarily conserved transcription factors of multiple classes. The forkhead class $\mathrm{O}$ (FoxO) members have emerged as important regulators of cellular metabolism and differentiation, survival and apoptosis, cell cycle arrest, autophagy, stress resistance and lifespan. The FoxO class is comprised of three main functionally-related proteins: FoxO1 (Galili et al., 1993), FoxO3 (Anderson et al., 1998; Hillion et al., 1997), and FoxO4 (Borkhardt et al., 1997) that are vertebrate orthologs of the Caenorhabditis elegans transcription factor DAF-16 (Lin et al., 1997; Ogg et al., 1997). A fourth member, FoxO6, has been identified by homology, is expressed only in brain, and its cellular localization is alternatively regulated (Jacobs et al., 2003).

FoxOs are regulated by multiple post-translational modifications: phosphorylation, acetylation, methylation, ubiquitination and O-linked glycosylation. These affect FoxO intracellular localization, transcriptional activity and turnover (Calnan and Brunet, 2008). FoxOs associate with many different cofactors, allowing them to regulate context-dependent gene expression programs (van der Vos and Coffer, 2008). Expression of FoxO-regulated genes can be controlled by any of the FoxO transcription factors and specificity is obtained either by their expression pattern or by isoform-specific regulation (van der Horst and Burgering, 2007).

A primary mode of FoxO regulation is negative control by the phosphoinositide 3-kinase (PI3K) pathway (FIG.4.1). Activation of PI3K by the insulin receptor results in production of phosphatidylinositol-(3,4,5)-triphosphate $\left(\mathrm{PIP}_{3}\right)$ (Sun et al., 1991), 
stimulating the protein kinase, Akt, to localize to the plasma membrane (Burgering and Coffer, 1995; Franke et al., 1995) where it is activated by the phosphoinositidedependent kinase, PDK1 (Alessi et al., 1997; Stokoe et al., 1997). Akt then phosphorylates FoxO members, which promotes their interaction with the adaptor protein 14-3-3 and leads to their exclusion from the nucleus (Rena et al., 2001; Brownawell et al., 2001; Brunet et al., 2002).

While FoxOs mediate gene expression with respect to a variety of cellular needs, one of the key functions of the FoxO proteins (that is of particular interest to this thesis) is the induction of quiescence by facilitating cell cycle arrest. As mentioned in Chapter 1, metabolic rate depression is arguably the most important factor in long-term anoxia tolerance and involves the coordinated shut down of many energy-expensive cell processes, major ones being cell proliferation and growth. The molecular mechanisms that mediate metabolic suppression in response to anoxia are of particular interest. Progression through the cell cycle is a tightly controlled process that is regulated by the balance between the amounts and activities of the cyclin-CDK (cyclin-dependent kinase) complexes and those of their inhibitors (Sherr and Roberts, 1999). FoxOs have been shown to block cell-cycle progression at the G1 phase by inducing the expression of the cell-cycle inhibitor, p27kip1 (Medema et al., 2000; Nakamura et al., 2000; Stahl et al., 2002; Tanaka et al., 2001). This inhibitor binds to the cyclin E-CDK2 complex and inhibits its activity, thereby preventing the entry of cells into the S phase (Reynisidottir et al., 1995). FoxOs also contribute to regulating exit from the cell cycle into a state of quiescence by inducing the expression of the retinoblastoma family member, p130 (Kops et al., 2002a). This protein is thought to be involved in the repression of genes required 
for reentry into the cell cycle, and therefore, its induction allows maintenance of a quiescent state (Smith et al., 1996). In addition, FoxOs can induce transcription of cyclin G2 (Martinez-Gac et al., 2004), a cyclin that is highly expressed in quiescent cells but is underexpressed as cells enter the cell cycle (Bennin et al., 2002; Horne et al., 1997). Another important FoxO function is to enhance cellular stress resistance. In $C$. elegans, induction of DAF-16 (the FoxO homologue) is associated with increased resistance to oxidative stress (Honda and Honda, 1999; Ogg et al., 1997), as well as extended lifespan (Katic and Kahn, 2005). DAF-16 was shown to undergo nuclear translocation in response to various types of oxidative stress (Henderson and Johnson, 2001). Similarly in mammals, oxidative stress caused by $\mathrm{H}_{2} \mathrm{O}_{2}$, menadione, or heat shock triggers the relocalization of FoxOs from the cytoplasm to the nucleus (Brunet et al., 2004; Kobayashi et al., 2005) and FoxOs induce the expression of antioxidant enzymes such as manganese-dependent superoxide dismutase (MnSOD) and catalase. In addition, FoxO3 upregulates expression of sterol carrier protein $\mathrm{x}$ (SCPx) and SCP2 (Dansen et al., 2004) that might be involved in protecting lipids against oxidative damage (Dansen et al., 2004). Another target gene of FoxO signaling is GADD45a, a protein that is induced in response to a variety of stressors, such as ionizing radiation, UV, and ROS and is involved in DNA-damage repair (Tran et al., 2002).

The present chapter examines the response of the FoxO transcription factors to anoxia in turtle tissues. Because of the potentially important roles that FoxOs play in hypometabolism, as well as other relevant processes such as stress resistance, I hypothesized that FoxO proteins would be activated in response to anoxia, potentially helping turtle cells achieve a hypometabolic state, as well as providing stress-resistance. 
This chapter examines the expression and activation of two members of the FoxO family, FoxO1 and FoxO3 and their phosphorylated forms, as well the expression of two target genes.

\subsection{MATERIALS AND METHODS}

\subsubsection{Animals}

Turtles were treated and tissue samples were collected as described in Chapter 2.

\subsubsection{RNA extraction and cDNA synthesis}

Total RNA was isolated from turtle tissues and cDNA was reverse transcribed and serially diluted as described in Chapter 2 .

\subsubsection{Primers}

Forward and reverse primers for foxol, foxo3, p27kipl and catalase were designed from conserved regions in these genes based on DNA alignment of the sequences from other vertebrate species. For foxol, foxo3 and p27kip1, perfect (turtlespecific) primers were designed and used for quantification after the turtle sequences for these genes were obtained. Primers for the control gene, $\alpha$-tubulin, were as described in chapter 3 . The sequences of the primers are listed in table 4.1

\subsubsection{RT-PCR}

PCR reactions were composed as described in Chapter 2. The PCR program was as follows: $7 \mathrm{~min}$ at $94^{\circ} \mathrm{C}$ followed by 35 cycles of $1 \min$ at $94^{\circ} \mathrm{C}, 1 \mathrm{~min}$ at a 
predetermined annealing temperature $\left(54^{\circ} \mathrm{C}\right.$ for catalase and tubulin, $62.5^{\circ} \mathrm{C}$ for foxol, foxo3 and p27kip1), and $1.5 \mathrm{~min}$ at $72^{\circ} \mathrm{C}$. The final step was $72^{\circ} \mathrm{C}$ for $10 \mathrm{~min}$. PCR products were separated on a $1 \%$ agarose gel stained with ethidium bromide, visualized using the ChemiGenius imaging system (Syngene, Frederick, MD, USA) under UV light and quantified using the GeneTools program. The bands from the most dilute cDNA sample that gave visible product were used for quantification to ensure that the products had not reached amplification saturation.

PCR products were sequenced by DNA Landmarks (St.-Jean-sur-Richelieu, QC) and sequences were verified as encoding the correct genes using the program BLASTN (http://www.ncbi.nlm.nih.gov/blast) at the NIH.

\subsubsection{Protein extracts and western blotting}

Protein extracts were prepared from tissue samples of aerobic, $5 \mathrm{~h}$ and $20 \mathrm{~h}$ anoxic turtles. Equal amounts of protein $(20-30 \mu \mathrm{g})$ were loaded into each lane of $10 \%$ SDS polyacrylamide gels, electrophoresed and then electroblotted onto polyvinylidene difluoride (PVDF) membranes as described in Chapter 2. After the transfer of proteins, membranes were blocked for $15 \mathrm{~min}$ in Tris buffered saline containing Tween-20 (TBST: $20 \mathrm{mM}$ Tris base, $140 \mathrm{mM} \mathrm{NaCl}, 0.1 \% \mathrm{v} / \mathrm{v}$ Tween-20) with $2.5 \%$ non-fat dried milk added. The blots were then probed overnight with primary antibodies for FoxO1 (Cell Signaling), p-FoxO1 (Ser 256) (Cell Signaling), FoxO3 (GenScript), p-FoxO3 (Ser 253) (GenScript), or p-FoxO3 (Ser 318/321) (Cell Signaling). All primary antibodies were diluted 1:1000 in TBST. After incubation, blots were washed several times with TBST and then incubated at RT for $1.5 \mathrm{~h}$ with HRP-linked goat anti-rabbit IgG (Cell Signaling, 
diluted 1:2000 in TBST). Immunoblots were developed using enhanced chemiluminescence reagents.

\subsubsection{Preparation of nuclear extracts}

Cytoplasmic and nuclear extracts from heart, kidney, liver and muscle of aerobic and anoxic turtles were prepared as described in Chapter 2.

\subsubsection{DNA-binding assay}

Nuclear extracts for DNA-binding assay were prepared as described in chapter 2. Aliquots containing equal amounts of protein from each sample (10-16 $\mu \mathrm{g} / \mathrm{well})$ were then used to assess the amount of binding by FoxO1 and FoxO3 to the FOXO element as described in Chapter 2. The sequence of the biotin-conjugated probe was 5'- BiotinGACTCGAGTT GTTTACATAGGATGCG-3', whereas the complementary sequence was 5'- CGCATCCTATGTAAACAACTCGAGTC- 3'.

For FoxO1, $16 \mu \mathrm{g}$ of protein was combined with $50 \mu \mathrm{L}$ of $1 \mathrm{x}$ protein binding buffer [10 mM Hepes, pH 7.9, $50 \mathrm{mM} \mathrm{KCl,} 0.5 \mathrm{mM}$ EDTA, $3 \mathrm{mM} \mathrm{MgCl} 2,10 \% \mathrm{v} / \mathrm{v}$ glycerol, $0.5 \mathrm{mg} / \mathrm{mL}$ BSA, $0.05 \% \mathrm{NP}-40,1 \mu \mathrm{g}$ salmon sperm DNA (BioShop), $0.5 \mathrm{mM}$ DTT, and $\mathrm{NaCl}$ (variable amount was used based on the volume of nuclear extract added, final concentration was always $40 \mathrm{mM}$ )]. The plate was incubated at RT with mild agitation for $75 \mathrm{~min}$ and then washed 4 times with wash buffer. Aliquots of $60 \mu \mathrm{L}$ of the FoxO1 antibody (same as used for western blotting), diluted 1:250 in PBS, were then added to the wells and the plate was incubated for $3 \mathrm{~h}$. The remainder of the procedure was as described in Chapter 2 . 
For FoxO3, $10 \mu \mathrm{g}$ of protein was combined with $50 \mu \mathrm{L}$ of $1 \times$ protein binding buffer (same as above, except that $2 \mu \mathrm{g}$ of salmon sperm DNA was used). The plate was incubated at RT with mild agitation for $75 \mathrm{~min}$ and then washed 4 times with wash buffer. Aliquots of $60 \mu \mathrm{L}$ of the FoxO3 antibody (same as used for western blotting), diluted 1:1000 in PBS, were added to the wells and the plate was incubated for $75 \mathrm{~min}$. The rest of the procedure was as described in Chapter 2.

\subsubsection{Data Analysis}

All data were analyzed as described in Chapter 2.

\subsection{RESULTS}

\subsection{1. cDNA cloning of turtle foxo1}

A PCR product of $632 \mathrm{bp}$ was retrieved using the original foxol primers (designed from the vertebrate consensus sequence) from total RNA prepared from turtle liver. The product was confirmed as encoding a portion of the foxol sequence by BLAST. FIG.4.2A shows the partial nucleotide sequence with the corresponding amino acid sequence. The partial turtle amino acid sequence was 210 amino acids in length, corresponding to amino acids $250-459$ of the chicken FoxO1 sequence and representing $30 \%$ of the total FoxO1 sequence. FIG.4.2B shows a homology tree produced from the alignment of the partial T. s. elegans FoxO1 protein sequence with FoxO1 from human (Homo sapiens), chicken (Gallus gallus), African clawed frog (Xenopus laevis) and zebrafish (Danio rerio). The chicken FoxO1 sequence was the closest to the turtle 
sequence, showing $96 \%$ similarity. Based on the homology matrix (not shown), the other vertebrates showed $81-94 \%$ similarity to the turtle sequence.

FIG. 4.3 shows the amino acid alignment of the partial T. s. elegans FoxO1 protein sequence with the other vertebrates. One unique amino acid substitution was observed in the turtle sequence, a leucine in the turtle sequence, compared to glutamic acid in the other animals.

\subsubsection{Transcript expression of foxol in response to anoxia}

Changes in the relative transcript levels of foxol in response to 5 and $20 \mathrm{~h}$ of anoxia were assessed using RT-PCR and perfect primers designed from the turtle sequence (FIG.4.4). FIG.4.4A shows representative RT-PCR bands of foxo1 amplicons with corresponding $\alpha$-tubulin bands, while FIG.4.4B shows a graphical representation of the changes in the relative levels of these transcripts in response to anoxia. Whereas foxol levels remained constant in the heart, kidney, and muscle throughout the anoxic episode, liver displayed significant increases in foxol transcripts. Levels increased to 2.4

\pm 0.4 fold higher than the aerobic control value after $5 \mathrm{~h}$ of anoxia and rose to $4.8 \pm 0.2$ fold of control after $20 \mathrm{~h}$ (both $\mathrm{P}<0.05$ ).

\subsubsection{Protein expression of FoxO1 and p-FoxO1 in response to anoxia}

Changes in the levels of the FoxO1 protein and its phosphorylated form (ser 256) in response to anoxia were assessed using immunobloting (FIG.4.5). The polyclonal antiFoxOl and anti-p-FoxO1 antibodies cross-reacted with a protein band of $\sim 70 \mathrm{kDa}$, the expected size of FoxO1. FIG. 4.5A shows the changes in the relative protein levels of 
FoxO1 in response to anoxia. Kidney, liver and muscle all displayed increases in FoxO1 levels in response to $5 \mathrm{~h}$ of anoxia, with levels rising to $1.8 \pm 0.2,1.8 \pm 0.1$, and $1.7 \pm 0.2$ fold of control (respectively; all $\mathrm{P}<0.05$ ). All returned to normoxic levels by $20 \mathrm{~h}$ of anoxia exposure. The heart was the only tissue that showed a decrease in FoxO1 levels, with levels falling to $54 \pm 6 \%$ of control in response to $5 \mathrm{~h}$ of anoxia $(\mathrm{P}<0.05)$ and returning to normoxic values by $20 \mathrm{~h}$ of anoxia.

FIG. 4.5B shows changes in the relative amount of $\mathrm{p}$-FoxOl in response to anoxia. p-FoxOl levels increased in both kidney and muscle in response to $5 \mathrm{~h}$ of anoxia by $1.7 \pm 0.2$ and $1.9 \pm 0.15$ fold, respectively, as compared with corresponding controls (both $\mathrm{P}<0.05$ ). Levels in both tissues returned to normoxic values by $20 \mathrm{~h}$ of anoxia. Liver showed the opposite response, a decrease to $57 \pm 3 \%$ of the control value in response to $5 \mathrm{~h}$ of anoxia exposure $(\mathrm{P}<0.05)$, but again returning to normoxic values by $20 \mathrm{~h}$. No change in p-FoxOl levels was observed in heart in response to anoxia.

\subsubsection{Nuclear expression and DNA-binding activity of FoxO1 in turtle tissues}

In order to determine whether FoxO1 translocated to the nucleus in response to anoxia, western immunoblotting was performed on nuclear extracts from turtle tissues (FIG.4.6A). The liver was the only tissue that showed a change in the nuclear levels of FoxO1, with FoxO1 levels increasing by $1.9 \pm 0.2$ fold in response to $5 \mathrm{~h}$ of anoxia exposure $(\mathrm{P}<0.05)$, returning to normoxic levels by $20 \mathrm{~h}$ of anoxia.

The DNA-binding activity of nuclear FoxO1 in response to anoxia was assessed only in the liver (FIG.4.6B), since this was the only tissue that showed an increased presence of FoxO1 in the nucleus. The DNA-binding activity of FoxO1 in liver nuclei 
increased by $3.8 \pm 0.7$ fold as compared with controls in response to $5 \mathrm{~h}$ of anoxia and then declined by about half (while still remaining elevated) to $2.1 \pm 0.1$ fold of the control value by $20 \mathrm{~h}$ of anoxia exposure (both $\mathrm{P}<0.05$ ).

\subsection{5. cDNA cloning of partial turtle foxo3}

A PCR product of $308 \mathrm{bp}$ was retrieved using the original foxo 3 primers from the total RNA prepared from turtle liver. The product was confirmed as encoding a portion of the foxo3 sequence by BLAST. FIG.4.7A shows the partial nucleotide sequence with the corresponding amino acid sequence. The sequence encoded 102 amino acids, corresponding to amino acids $389-480$ of the chicken FoxO3 sequence and representing 15\% of the full FoxO3 sequence. FIG.4.7B shows a homology tree produced from the alignment of the partial T. s. elegans FoxO3 protein sequence with FoxO3 from human (H. sapiens), chicken (G. gallus), African clawed frog (X. laevis) and zebrafish (D. rerio). The chicken FoxO1 sequence was the closest to the turtle sequence, showing a $93 \%$ similarity. According to the homology matrix (not shown) the other vertebrates showed $57-85 \%$ similarity to the turtle sequence.

FIG. 4.8 shows the amino acid alignment of the partial T. s. elegans FoxO1 protein sequence with the other vertebrates. One unique amino acid substitution was observed in the turtle sequence, a proline in the turtle sequence, compared to serine in the other animals.

\subsubsection{Transcript expression of foxo3in response to anoxia}

Changes in the relative transcript levels of foxo 3 in response to 5 and $20 \mathrm{~h}$ of 
anoxia were assessed using RT-PCR and perfect (species-specific) primers designed from the turtle sequence (FIG.4.9). FIG.4.9A shows representative RT-PCR bands of foxo 3 amplicons with corresponding $\alpha$-tubulin bands, whereas FIG.4.9B shows a histogram of the mean normalized transcript levels. Transcript levels of foxo 3 did not change significantly in response to anoxia in any of the tissues examined.

\subsubsection{Protein expression of FoxO3 and p-FoxO3 in response to anoxia}

Changes in the amount of FoxO3 protein and its phosphorylated forms (ser 253, ser 318/321) in response to anoxia were assessed using immunobloting (FIG.4.10). The polyclonal anti-FoxO3 and anti-p-FoxO3 antibodies crossreacted with a protein band at $\sim 75 \mathrm{kDa}$, the expected size of FoxO3. FIG. 4.10A shows changes in the relative protein levels of FoxO3 in response to anoxia. In heart, kidney, and muscle FoxO3 remained stable in response to $5 \mathrm{~h}$ of anoxia, but levels increased by $1.3 \pm 0.1,2.7 \pm 0.3$ or $3.1 \pm$ 0.5 fold respectively as compared with controls in response to $20 \mathrm{~h}$ of anoxia (all $\mathrm{P}<$ 0.05). No changes in FoxO3 levels were observed in liver.

FIG. 4.10B shows the data for p-FoxO3 (Ser 253). Levels of p-FoxO3 (Ser 253) in the heart were unchanged after $5 \mathrm{~h}$ of anoxia, but showed a slight increase to $1.3 \pm 0.03$ fold of control values in response to $20 \mathrm{~h}$ of anoxia $(\mathrm{P}<0.05)$. In skeletal muscle, $\mathrm{p}$ FoxO3 (Ser 253) levels were also stable after $5 \mathrm{~h}$ of anoxia, but significantly increased to $1.7 \pm 0.06$ fold of controls in response to $20 \mathrm{~h}$ anoxia $(\mathrm{P}<0.05)$. Liver also showed no change after $5 \mathrm{~h}$ but p-FoxO3 (Ser 253) levels decreased significantly to $64 \pm 6 \%$ of the control value after $20 \mathrm{~h}$ of anoxia $(\mathrm{P}<0.05)$. No changes in $\mathrm{p}$-FoxO3 (Ser 253) content were seen in kidney after either length of anoxia exposure. 
FIG. 4.10C shows the changes in the relative amounts of p-FoxO3 (Ser 318/321) in response to anoxia. Levels of this phosphorylated form decreased significantly in response to anoxia in the heart and kidney. In heart, levels fell to $39 \pm 14 \%$ and $30 \pm 4 \%$ of control values in response to $5 \mathrm{~h}$ and $20 \mathrm{~h}$ anoxia, respectively (both $\mathrm{P}<0.05$ ). Similarly, the relative amount of p-FoxO3 (Ser 318/321) in kidney was reduced to $57 \pm$ $17 \%$ and $52 \pm 9 \%$ of controls in response $5 \mathrm{~h}$ and $20 \mathrm{~h}$, respectively (both $\mathrm{P}<0.05$ ). In the liver, p-FoxO3 (Ser 318/321) content rose by $1.8 \pm 0.07$ fold after $5 \mathrm{~h}$ of anoxia and remained elevated at $1.4 \pm 0.04$ fold over controls after $20 \mathrm{~h}$ of anoxia $(\mathrm{P}<0.05)$. In the muscle, no significant change was observed after $5 \mathrm{~h}$ anoxia, but p-FoxO3 (Ser 318/321) content significantly increased to $2.1 \pm 0.3$ fold of control values after $20 \mathrm{~h}$ of anoxia $(\mathrm{P}<$ $0.05)$.

\subsubsection{Nuclear expression and DNA-binding activity of FoxO3 in turtle tissues}

In order to determine whether FoxO3 translocated to the nucleus in response to anoxia, western immunoblotting was performed on nuclear extracts from turtle tissues (FIG.4.11A). Heart, kidney and liver all displayed significant increases in nuclear FoxO3 content in response to anoxia. In heart nuclei, FoxO3 levels were stable after $5 \mathrm{~h}$ of anoxia, but significantly increased to $1.5 \pm 0.1$ fold higher than controls in response to 20 $\mathrm{h}$ of anoxia $(\mathrm{P}<0.05)$. In the kidney, nuclear FoxO3 increased to $2.4 \pm 0.3$ fold of control values after $5 \mathrm{~h}$ anoxia and to $3.7 \pm 0.9$ fold of control by $20 \mathrm{~h}$ anoxia $(\mathrm{P}<0.05)$. In liver, nuclear FoxO3 levels were $2.5 \pm 0.3$ and $2.6 \pm 0.4$ fold higher than controls at 5 and $20 \mathrm{~h}$ of anoxia, respectively ( $\mathrm{P}<0.05$ for both). No changes in the nuclear levels of FoxO3 were observed in the muscle. 
Changes in the DNA-binding activity of nuclear FoxO3 in response to anoxia were assessed using a transcription factor binding assay for heart, kidney, and liver (FIG.4.11B). The pattern mirrored that seen for nuclear FoxO3 content in FIG.4.11A. Heart showed no change in the DNA-binding activity by FoxO3 in nuclear extracts after $5 \mathrm{~h}$ anoxia, but a $1.4 \pm 0.1$ fold increase was observed in response to $20 \mathrm{~h}(\mathrm{P}<0.05)$. In the kidney, the DNA-binding activity of FoxO3 increased to $1.3 \pm 0.05$ and $1.6 \pm 0.04$ fold of control values after 5 and $20 \mathrm{~h}$ of anoxia, respectively (both $\mathrm{P}<0.05$ ). Similarly, liver nuclear extracts showed $2.9 \pm 0.2$ and $2.6 \pm 0.1$ fold increases in DNA-binding activity of FoxO3 after 5 and 20 h of anoxia, respectively (both $\mathrm{P}<0.05$ ).

\subsection{9. cDNA cloning of partial turtle p27kip1}

A PCR product of $637 \mathrm{bp}$ was retrieved using the original p27kipl primers from the total RNA prepared from turtle liver. The product was confirmed as encoding a portion of the $27 k i p 1$ sequence by BLAST. This sequence included the stop codon for the p27kipl protein (underlined and bolded in the figure). FIG.4.12A shows the partial nucleotide sequence with the translated 149 amino acid sequence. This corresponded to amino acids $50-198$ of the chicken p27kipl sequence and represented $75 \%$ of the total p27kipl sequence including the entire C-terminus of the protein. FIG.4.12B shows a homology tree produced from the alignment of the partial T. s. elegans p27kip1 protein sequence with p27kipl from human (H. sapiens), chicken (G. gallus) and zebrafish (D. rerio). The chicken p27kipl sequence was the closest to the turtle sequence, showing $80 \%$ similarity. 


\subsubsection{Transcript expression of 2 27kiplin response to anoxia}

Relative transcript levels of p27kipl in control, 5 and $20 \mathrm{~h}$ anoxic turtles are shown in FIG.4.13. Representative RT-PCR bands of p27kipl amplicons with corresponding $\alpha$-tubulin bands are shown in FIG.4.13A, whereas FIG.4.13B shows the histogram with mean transcript levels. Levels of p27kip 1 transcripts did not change in response to anoxia in heart or kidney. In liver, however, $p 27 k i p 1$ levels rose to $2.5 \pm 0.2$ fold above control in response to $5 \mathrm{~h}$ of anoxia $(\mathrm{P}<0.05)$, returning to normoxic levels by $20 \mathrm{~h}$.

\subsubsection{3. cDNA cloning of partial turtle catalase}

A PCR product of $400 \mathrm{bp}$ was retrieved using the original catalase primers and was confirmed as encoding a portion of the catalase sequence by BLAST. FIG.4.14A shows the partial nucleotide sequence and the corresponding 133 amino acid sequence. This sequence corresponded to amino acids $85-216$ of the chicken catalase sequence and represents $25 \%$ of the full catalase sequence. FIG.4.14B shows the homology tree for the partial T. s. elegans catalase amino acid sequence in comparison with catalase from human (H. sapiens), chicken (G. gallus), African clawed frog (X. laevis) and zebrafish (D. rerio). Chicken catalase was the closest to the turtle sequence, showing a $97 \%$ similarity.

\subsubsection{Transcript expression of catalase in response to anoxia}

FIG.4.15 shows the relative transcript levels of catalase in tissues of control, $5 \mathrm{~h}$ 
and $20 \mathrm{~h}$ anoxic turtles. Representative RT-PCR bands for catalase amplicons and corresponding $\alpha$-tubulin bands are shown in FIG.4.15A, and FIG.4.15B shows mean relative transcript levels. Transcript levels of catalase did not change in response to anoxia in heart or kidney. However, liver showed a $2.4 \pm 0.4$ fold increase in transcripts in response to $5 \mathrm{~h}$ of anoxia $(\mathrm{P}<0.05)$ but levels returned to normoxic values by $20 \mathrm{~h}$ of anoxia.

\subsection{DISCUSSION}

The FoxO proteins are an important family of transcription factors that regulate many programs of gene expression involved in cellular metabolism, apoptosis, cell cycle and stress resistance. The best-known mechanism of FoxO regulation is control by insulin and various related growth factors via the PI3K pathway that activates the protein kinase Akt which subsequently phosphorylates FoxOs, resulting in their nuclear exclusion (FIG.4.1) (Sun et al., 1991; Burgering and Kops, 2002). However, in the absence of PI3K activation, or in the presence of stress, FoxOs become dephosphorylated and activated. In C. elegans, FoxO activation is concurrent with hypometabolism, since an absence of Akt signaling leads to activation of the FoxO homologue, DAF-16, and entrance into the quiescent dauer state (Ogg et al., 1997; Paradis et al., 1998). In addition, studies in mammalian cells have shown that activation of FoxO proteins is associated with survival under fasting conditions by facilitating cell cycle arrest (van der Horst and Burgering, 2007). Various studies have also shown that FoxOs are activated in response to oxidative stress and mediate oxidative stress resistance (van der Horst and Burgering, 2007). In mammalian cells, FoxOs are also activated in response to 
hypoxia/ischemia (Zhan et al., 2010; Bakker et al., 2007) and protect cells against these stresses.

Survival of anoxia by facultative anaerobes includes both a profound metabolic rate depression, as well as activation of various stress-responsive pathways. Owning to the involvement of the FoxO proteins in hypometabolism, as well as other cellular processes required for anoxic survival, I hypothesized that FoxO signaling would be activated in turtle tissues in response to anoxia. The present chapter examined the expression and activation of FoxO1 and FoxO3, as well as several target genes, in response to anoxic submergence in $T$. $s$. elegans. The data show that activation of these transcription factors occurs in a tissue-specific manner in response to anoxia and suggest that they have an important regulatory role in anoxia-responsive gene expression in this species.

FoxO1 and FoxO3 sequence analysis

Partial sequences for both FoxO1 and FoxO3 were obtained from T. s. elegans (FIG. 4.2 and FIG. 4.7, respectively) liver and aligned with sequences from other vertebrates for comparison. Both sequences were fairly highly conserved, turtle FoxO1 and FoxO3 showing $96 \%$ and $93 \%$ similarity (respectively) to the chicken sequences. Among the vertebrates examined, the chicken sequence was the one most closely related to the turtle FoxOs, indicating the closest evolutionary relationship between these two animals.

FIG.4.3 and FIG.4.8 show the amino acid alignments of the partial FoxOl and FoxO3 sequences compared to the other vertebrates. The FoxO1 sequence had one 
unique amino acid substitution, a leucine in the turtle compared to glutamic acid in the other vertebrates. These amino acids are quite different in structure and properties, leucine being hydrophobic and with a nonpolar side chain and a neutral charge whereas glutamic acid is hydrophilic and has a polar side chain with a hydrophilic charge. The FoxO3 amino acid alignment also revealed one turtle-specific amino acid substitution, a proline in the turtle compared to a serine in the other vertebrates. While both of these amino acids are neutral and hydrophobic, they are structurally distinct and have side chains of different polarity (proline is non-polar and serine is polar). Therefore, it is possible that this substitution could affect the structure of the FoxO3 protein. Overall, the substitutions seen could lead to some structural differences between turtle FoxOs and FoxOs from other vertebrates and could potentially point to a turtle-specific adaptation that would stabilize this protein under the low-pH and altered redox conditions that characterize anoxia.

\section{Activation of FoxO1 in response to anoxia}

In order to study the activation of FoxO1 in response to anoxia, transcript levels, protein levels, nuclear translocation, and DNA-binding activity were analyzed in different turtle tissues. Results from RT-PCR and western immunoblotting suggested that FoxO1 is regulated on multiple levels in response to anoxia. Liver was the main tissue that displayed FoxO1 activation. FoxO1 transcript levels increased by 2.4-4.8-fold in response to 5 and $20 \mathrm{~h}$ of anoxia (FIG. 4.4), while protein levels increased by 1.8-fold (FIG.4.5A) after $5 \mathrm{~h}$, suggesting that transcriptional upregulation is an important part of FoxO1 activation in response to anoxia in this tissue. In addition to the increase in 
FoxO1 protein levels, the relative amount of phosphorylated FoxO1 (Ser256) decreased to $57 \%$ of the control value (FIG.4.5B). Phosphorylation of FoxO1 on this residue is mediated by Akt and is important for nuclear exclusion as well as suppression of transactivation of FoxO1 (Rena et al., 2001, 2002; Guo et al., 1999; Zhang et al., 2002a). Therefore, a reduction in the amount of the phosphorylated form, combined with the increase in the total FoxO1 protein suggests activation and nuclear translocation of this transcription factor might be occurring. Indeed, a 1.9-fold increase in FoxO1 levels was observed in liver nuclei in response to $5 \mathrm{~h}$ anoxia (FIG.4.6A). This increase in nuclear FoxO1 content was also correlated with a 3.9-fold increase in the DNA-binding activity of FoxO1 in liver nuclei (FIG.4.6B). This strong increase in binding activity, together with the observation that DNA-binding activity remained high (2.1-fold over control) at $20 \mathrm{~h}$ anoxia despite a decrease in nuclear FoxO1 content back to control levels (FIG.4.6B), suggests that additional mechanisms might be activated to enhance the DNA-binding activity of FoxO1 in response to anoxia. For example, it has been shown that in addition to phosphorylation, the transcriptional activities of FoxOs can be controlled by acetylation, methylation and ubiquitination (Calnan and Brunet, 2001).

Both kidney and skeletal muscle displayed 1.7-1.8 fold increases in FoxO1 protein levels after $5 \mathrm{~h}$ of anoxia exposure, despite a lack of change in transcript levels (FIG.4.4 and 4.5A). This result might be due to enhanced stabilization of the FoxO1 protein, since FoxO levels also appear to be regulated via controls on protein degradation (Calnan and Brunet, 2008). FoxOs can be polyubiquitinated and degraded in a proteasome-dependent manner in response to insulin and growth factors (Matsuzaki et al., 2003; Plas and Thompson, 2003). In contrast, in response to oxidative stress, 
acetylation of FoxO1 has been shown to increase FoxO stability by preventing polyubiquitination (Kitamura et al., 2005). Therefore, a decrease in FoxO polyubiquitination in response to anoxia could potentially be an energy-effective strategy to enhance FoxO protein levels. However, despite elevated amounts of total FoxO1 protein, phosphorylation at Ser 256 was enhanced by 1.7-1.9 fold in $5 \mathrm{~h}$ anoxic turtles, and no changes in nuclear FoxO1 levels were observed, strongly suggesting that FoxO1 transcriptional activity does not increase in these tissues in response to anoxia.

\section{Activation of FoxO3 in response to anoxia}

A tissue-specific activation pattern was also observed for $\mathrm{FoxO} 3$ in response to anoxia. FoxO3 was shown to be fully activated in the kidney. While transcript levels did not change, FoxO3 protein levels increased by 2.7 -fold in response to $20 \mathrm{~h}$ of anoxia (FIG. 4.9 and 4.10), probably due to increased protein stability. In addition, whereas phosphorylation of FoxO3 on Ser 253 was stable in response to anoxia, phosphorylation on serine $318 / 321$ decreased significantly, to $52-57 \%$ of control (FIG. 4.10). Phosphorylation of FoxO3 on serines 253,318 and 321 is mediated by Akt and facilitates the association of FoxO3 with the 14-3-3 proteins, resulting in nuclear exclusion (Brunet et al., 1999; Arden, 2004). Therefore, as in the case of FoxO1, it is expected that reduced phosphorylation on one or more of these residues, combined with enhanced protein levels, would elevate nuclear translocation. Indeed, strong enrichment of FoxO3 in the nucleus was observed, with levels in kidney increasing by 2.4 fold in response to $5 \mathrm{~h}$ of anoxia and rising to 3.7 fold higher than controls after $20 \mathrm{~h}$ anoxia (FIG. 4.11A). A 1.31.6 fold increase in the DNA-binding activity of FoxO3 was also observed (FIG. 4.11B), 
further confirming activation of this transcription factor in kidney of anoxia-exposed turtles.

While the liver also displayed a strong activation of FoxO3 in anoxia, a somewhat unusual pattern was observed. No changes were seen in either transcript or total protein levels of FoxO3 in response to anoxia (FIG. 4.9 and 4.10) but the phosphorylated FoxO3 forms were anoxia-responsive. The amount of p-FoxO3 (Ser253) decreased significantly to $64 \%$ of control after $20 \mathrm{~h}$ of anoxia but the amount of p-FoxO3 (Ser318/321) increased by1.4-1.8 fold (FIG. 4.10). However, despite this increase, FoxO3 still showed enhanced nuclear translocation in anoxic liver, with levels increased by 2.5-2.6 fold (FIG. 4.11A). This correlated with 2.6-2.9 fold increases in the DNA-binding activity of FoxO3 in liver nuclear extracts from anoxia-exposed animals (FIG. 4.11B). This activation pattern was somewhat unexpected since increased phosphorylation of $\mathrm{FoxO} 3$ is expected to lead to nuclear exclusion (and while phosphorylation on serine 253 did decrease, this was not observed until $20 \mathrm{~h}$ ). However, it has been shown that stress stimuli can trigger the relocalization of FoxOs into the nucleus even in the presence of growth factors and FoxO phosphorylation (Brunet et al., 2004; Kitamura et al., 2005; Frescas et al., 2005). This can occur due to monoubiquitination of FoxOs, or phosphorylation by stress-responsive kinases such as MST1 or JNK which “override" the effects of phosphorylation by Akt and lead to nuclear localization (van der Horst et al., 2006; Lehtinen et al., 2006; Essers et al., 2004; Oh et al., 2005). Therefore, it is possible that these kinases become activated in response to anoxia in turtle liver, overriding the effects of enhanced phosphorylation at Ser318/321 and allowing activation of FoxO3.

The heart showed a similar, albeit milder, response to anoxia. FoxO3 transcript 
levels remained stable, whereas FoxO3 protein levels showed a slight increase of 1.3 fold after $20 \mathrm{~h}$ of anoxia. Interestingly, the two phosphorylated forms of FoxO3 showed opposite responses, with p-FoxO3 (Ser253) content increasing by 1.3 -fold after $20 \mathrm{~h}$ of anoxia while p-FoxO3 (Ser318/21) decreased to $30-40 \%$ of control values in anoxia (FIG. 4.10). Despite the small increase in phosphorylation on Ser253, FoxO3 still appeared to become activated, with nuclear levels rising by 1.5 fold and DNA-binding activity rising by 1.4 fold in response to $20 \mathrm{~h}$ of anoxia (FIG. 4.11).

Skeletal muscle displayed yet another pattern of activation with respect to FoxO3. Total protein levels, as well as amounts of both phosphorylated forms increased by 1.73.1 in response to $20 \mathrm{~h}$ of anoxia (FIG. 4.10). However, FoxO3 content in the nucleus was unchanged, likely due to the enhanced phosphorylation of FoxO3. A possible explanation for this odd pattern could be that FoxO3 is not actually necessary for anoxia survival in this tissue, but is rather induced in preparation for recovery, where it could play a protective role against oxidative damage.

All of these results suggest an important role for FoxO proteins in anoxia tolerance of turtle tissues, in particularly liver. To further determine whether activation of FoxOs in response to anoxia correlated with upregulation of FoxO-responsive target genes, anoxia-responsive changes in the transcript levels of $\mathrm{p} 27 \mathrm{kipl}$ and catalase were measured next.

\section{Expression of $27 k i p 1$ in response to anoxia}

One of the major functions of FoxOs is the promotion of a quiescent state by inducing genes involved in cell cycle arrest. A key FoxO target gene is p27kip1 and 
FoxOs have been shown to block cell cycle progression at the G1 phase by inducing the expression of this protein (Medema et al., 2000; Nakamura et al., 2000; Stahl et al., 2002; Tanaka et al., 2001). This cell cycle inhibitor functions by binding to the cyclin ECDK2 complex and inhibiting its activity, thereby preventing the entry of cells into the $\mathrm{S}$ phase (Reynisidottir et al., 1995).

A partial amino acid sequence was obtained for p27kip1 from T. s. elegans (FIG. 4.12) and aligned with the protein from other vertebrates. As in the case of most proteins examined in this thesis, turtle p27kip1 was most closely related to the chicken sequence, showing $80 \%$ similarity (FIG. 4.12). Among the tissues examined, only liver showed a significant change in $227 \mathrm{kip} 1$ transcript levels, rising by 2.5 fold in response to $5 \mathrm{~h}$ of anoxia (FIG. 4.13). This upregulation correlates with the strong activation of both FoxO1 and FoxO3 seen in this tissue, suggesting that it was FoxO-mediated. A strong suppression of metabolic rate has long been considered to be the most important factor in anoxia tolerance, since it lowers metabolic requirements and allows tissues to survive using anaerobic glycolysis alone to generate ATP. Previous studies have shown significant metabolic rate depression in turtle hepatocytes in response to anoxia, including a overall $94 \%$ reduction in ATP turnover and an almost total halt to protein synthesis (a necessity for the cell cycle) (Hochachka et al., 1996). Upregulation of p27kip1 in a FoxO-specific manner, and the subsequent cell cycle arrest that it would help to impose, would strongly lower the metabolic demands of liver and contribute to establishment of the hypometabolic state. 


\section{Transcript expression of catalase in response to anoxia}

The second FoxO target gene that was examined in this chapter is catalase, a protein that plays a pivotal role in cellular antioxidant defenses and is involved in the detoxification of hydrogen peroxide by converting it to water and oxygen. As mentioned previously, the electron carriers of the respiratory chain become highly reduced during anoxia, which could lead to a rapid generation of reactive oxygen species (ROS) upon recovery. Anoxia-inducible expression of various antioxidant proteins appears to be an important preparatory mechanism for this stress. In addition, enhancement of antioxidant defenses during periods of prolonged anoxia can also be important for cell preservation during hypometabolism. As part of the energy-savings of metabolic rate depression, all ATP-expensive biosynthetic processes are strongly suppressed which means that replacement of damaged proteins (such as those damaged by ROS) is greatly curtailed and that, to remain viable, cells must greatly extend the half-life of their macromolecules. One way to do this is to enhance defense and preservation mechanisms that maintain proteins in native functional states for as long as possible. This includes actions such as elevating antioxidant defenses to deal with any ROS and increasing amounts of chaperone proteins to refold proteins that under normal aerobic circumstances might instead be degraded.

The partial amino acid sequence of turtle catalase was aligned with the sequences from other vertebrates and was found to have a $97 \%$ similarity with the chicken sequence (FIG. 4.14). As in the case of the other FoxO target gene examined, the expression of catalase only increased significantly in the liver, increasing by 2.4 fold in response to $5 \mathrm{~h}$ of anoxia (FIG.4.15). Incidentally, these results correlate with results for transcripts of 
other antioxidant proteins (see Chapter 3), suggesting that inducible antioxidant defenses play a particularly important role in the liver during anoxia-reoxygenation bouts.

\section{Conclusions}

Overall, the present chapter suggests an important role for the FoxO transcription factors in anoxia tolerance of $T$. s. elegans, showing that FoxOs are activated in a tissuespecific manner in response to anoxia. The soft tissues, liver, and kidney, showed the most prominent activation, whereas the heart showed a lesser activation of FoxO3. Transcripts of both of the FoxO target genes examined were elevated in the liver in response to anoxia, further stressing the importance of FoxO-mediated gene regulation to anoxia survival in this tissue. 
TABLE 4.1. List of primers used in chapter 4

\begin{tabular}{|c|c|c|c|}
\hline Gene/primer & Primer sequence (5'-3') & $\begin{array}{c}\begin{array}{c}\text { Annealing } \\
\text { temp. }\end{array} \\
\left({ }^{\circ} \mathrm{C}\right) \\
\end{array}$ & $\begin{array}{c}\begin{array}{c}\text { Expected } \\
\text { product size }\end{array} \\
\text { (bp) } \\
\end{array}$ \\
\hline $\begin{array}{l}\text { foxol original } \\
\text { forward }\end{array}$ & AACCTGTCCTACGCSGACCT & \multirow[t]{2}{*}{62.5} & \multirow[t]{2}{*}{$\sim 700$} \\
\hline $\begin{array}{l}\text { foxol original } \\
\text { reverse }\end{array}$ & GCAGGTGASGACTGKGTTGA & & \\
\hline $\begin{array}{l}\text { foxol perfect } \\
\text { forward }\end{array}$ & GGTGCAGAATGAAGGAACAG & \multirow[t]{2}{*}{62.5} & \multirow[t]{2}{*}{$\sim 500$} \\
\hline $\begin{array}{l}\text { foxol perfect } \\
\text { reverse }\end{array}$ & GGTTGATACGGTCAGTGATG & & \\
\hline $\begin{array}{l}\text { foxo } 3 \text { original } \\
\text { forward }\end{array}$ & GAGCTGGATGCCTGGACAGA & \multirow[t]{2}{*}{62.5} & \multirow[t]{2}{*}{$\sim 500$} \\
\hline $\begin{array}{l}\text { foxo } 3 \text { original } \\
\text { reverse }\end{array}$ & GCSTGCTTGTTCTCCTGGAT & & \\
\hline $\begin{array}{l}\text { foxo3 perfect } \\
\text { forward }\end{array}$ & AATAGTCCATCAAGCATGTC & \multirow[t]{2}{*}{62.5} & \multirow[t]{2}{*}{$\sim 200$} \\
\hline $\begin{array}{l}\text { foxo3 perfect } \\
\text { reverse }\end{array}$ & GAGAACCAAGTCCGGAACCT & & \\
\hline $\begin{array}{l}\text { p27kipl original } \\
\text { forward }\end{array}$ & GCCTGCAGRAACCTCTTCGG & \multirow[t]{2}{*}{62.5} & \multirow[t]{2}{*}{$\sim 700$} \\
\hline $\begin{array}{l}\text { p27kipl original } \\
\text { reverse }\end{array}$ & AATGCTACAT CCRAYGCTTT & & \\
\hline $\begin{array}{l}\text { p27kipl perfect } \\
\text { forward }\end{array}$ & CCTGGCTGGCAAGTTCGAGT & \multirow[t]{2}{*}{62.5} & \multirow[t]{2}{*}{$\sim 400$} \\
\hline $\begin{array}{l}\text { p27kip1 perfect } \\
\text { reverse }\end{array}$ & ACCACCGAGGCATCCGATGA & & \\
\hline catalase forward & GAGAGMGGATTCCTGARAGAG & \multirow[t]{2}{*}{54} & \multirow[t]{2}{*}{$\sim 500$} \\
\hline catalase reverse & GATCCRTAKCCATTCATRTG & & \\
\hline
\end{tabular}


FIG. 4.1

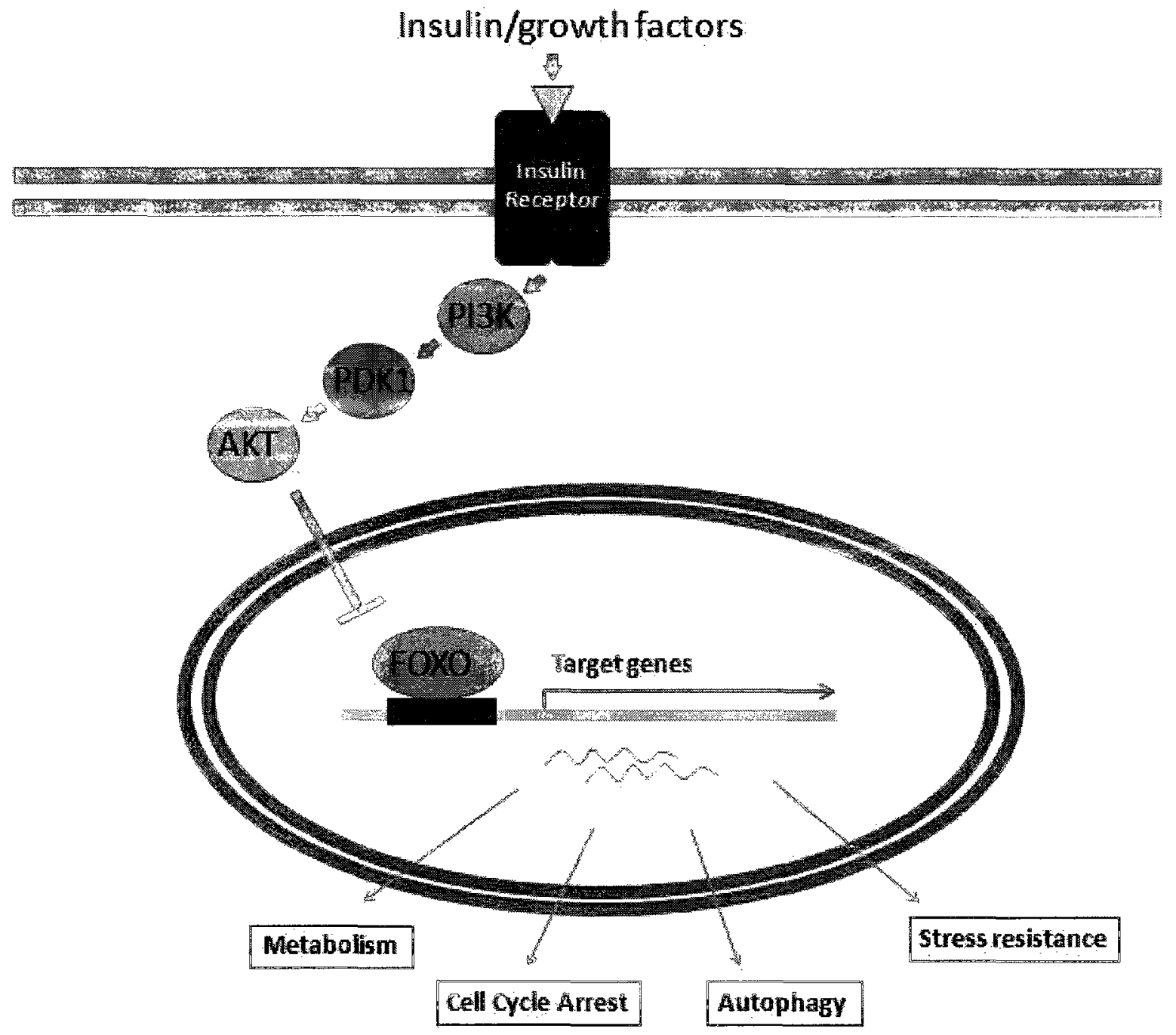

FIG.4.1. An overview of the FoxO pathway. In the presence of insulin or growth factors, the PI3K pathway is activated. This leads to activation of the protein kinase Akt, which phosphorylates FoxO family members, resulting in their nuclear exclusion and inhibition. In the absence of insulin and growth factors, FoxOs become activated and regulate a variety of gene expression programs associated with metabolism, cell cycle arrest, stress resistance and autophagy. 


\section{FIG. 4.2}

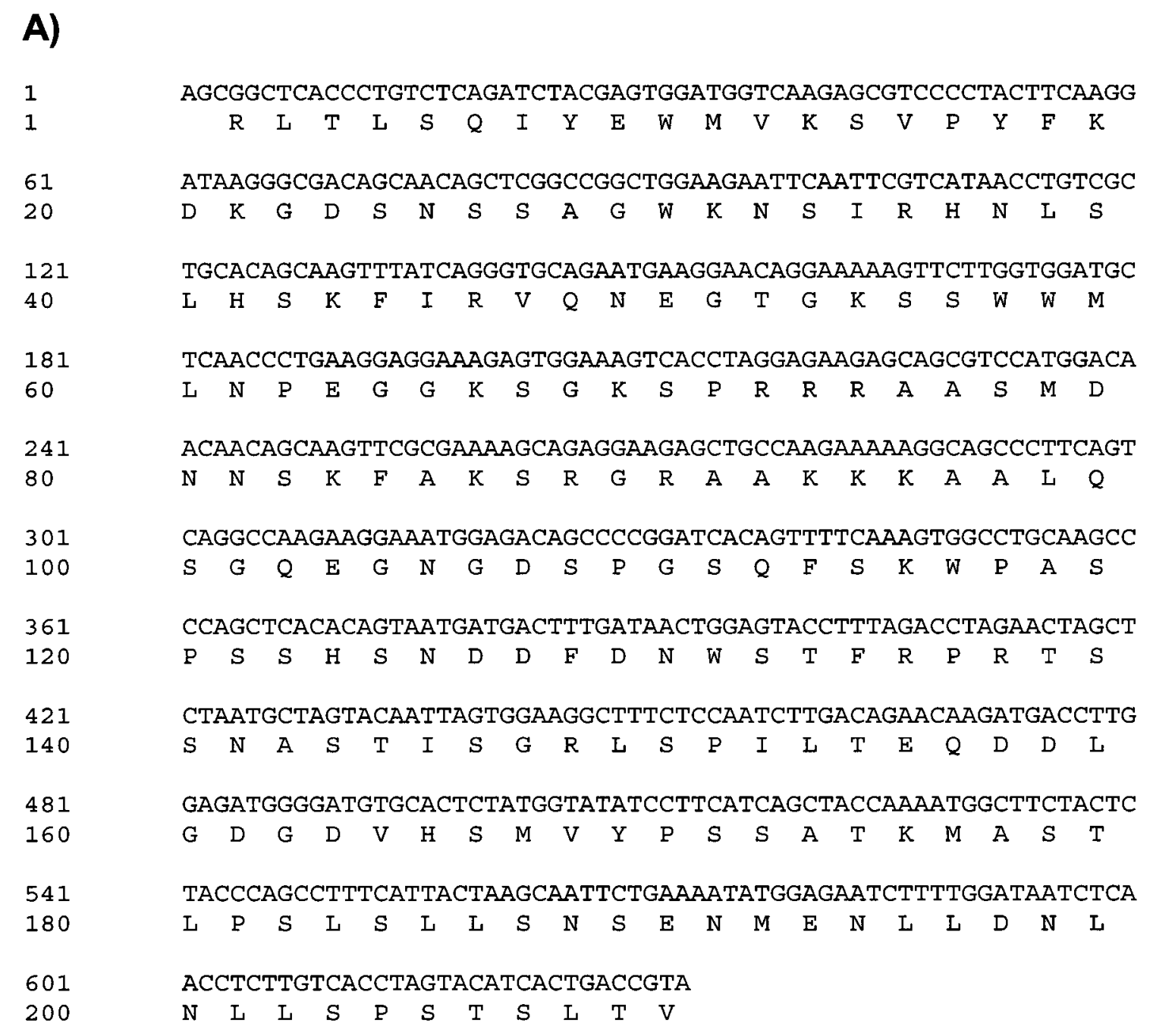

B)

$100 \% 95 \% 90 \% 85 \% 80 \% 75 \% \quad 70 \% 65 \% \quad 60 \%$
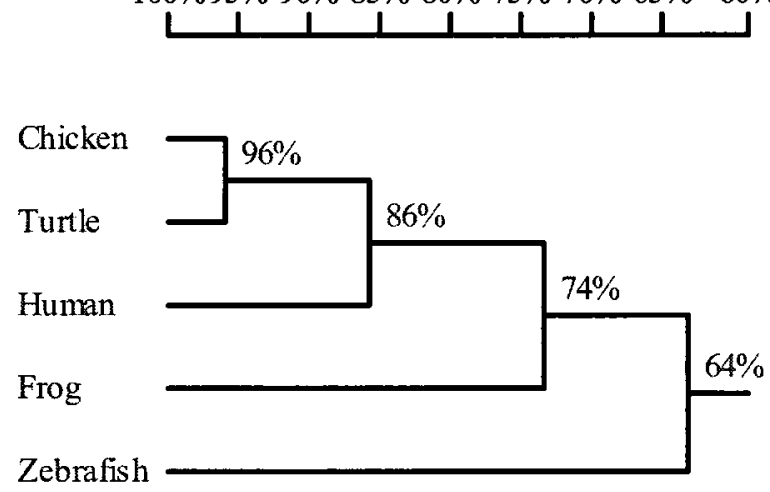
FIG. 4.2. Partial nucleotide and deduced amino acid sequence of $T$. s. elegans FoxO1.

(A) The partial cDNA sequence of foxol, with the corresponding amino acid sequence is shown. Nucleotides and amino acids are numbered on the left. The nucleotide sequence was 632 nucleotides in length, and encoded 210 amino acids. This sequence covered amino acids $250-459$ of the chicken FoxO1 sequence, representing $\sim 30 \%$ of the total FoxO1 protein.

(B) Homology tree produced from an alignment of the partial T. s. elegans FoxO1 protein sequence with FoxO1 from chicken (Gallus gallus), human (Homo sapiens), African clawed frog (Xenopus laevis) and zebrafish (Danio rerio). Accession numbers are NP_989659 for chicken, NP_002006 for human, NP_001086417 for frog and NP_001070725 for zebrafish. The percentage values correspond to the shared identity among the corresponding species. 
FIG. 4.3

\begin{tabular}{|c|c|c|}
\hline T. s. elegans & $\ldots \ldots \ldots \ldots \ldots \ldots \ldots \ldots \ldots \ldots \ldots \ldots \ldots \ldots \ldots \ldots \ldots \ldots$ & 7 \\
\hline G. gallus & gqprkssssrrnawgnlsyadlitkaiesspek------ & 256 \\
\hline H. sapiens & gqprkssssrrnawgnlsyadli tkaiessaek-- - - - - & 186 \\
\hline X. Iaevis & qqprkssssrrnawgnlsyadlisqaiesspek---- - - & 175 \\
\hline D. rerio & aaqrkssssrrnawgnmsyadlitkaiesspek------ & 160 \\
\hline T. s. elegans & YEWMVKSVPYFKDKGDSNSSAGWKNSIRHNLSLHSKFIRV & 47 \\
\hline G. gallus & ---------------------------------------- & 296 \\
\hline H. sapiens & ---------------------------------------- & 226 \\
\hline X. Iaevis & $-\mathrm{d}-----------------------------------v--$ & 215 \\
\hline D. rerio & $-\mathrm{d}---------------------------------r----$ & 200 \\
\hline T. s. elegans & QNEGTGKSSWWMLNPEGGKSGKSPRRRAASMDNNSKFAKS & 87 \\
\hline G. gallus & ---------------------------------------- & 336 \\
\hline H. sapiens & 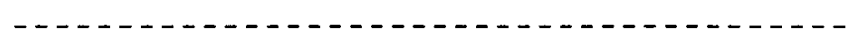 & 266 \\
\hline$X$. laevis & $-----------i-------n--------------------$ & 255 \\
\hline D. rerio & 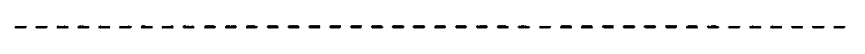 & 240 \\
\hline s. elegans & RGRAAKKKAALQSGQEGNGDSPGSQFSKWPASPSSHSNDD & 127 \\
\hline G. gallus & 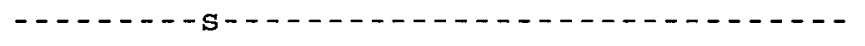 & 376 \\
\hline H. sapiens & $-s------s-------a--------------g-----$ & 306 \\
\hline X. laevis & $-------s m--s-d-s s--------g---q---$ & 295 \\
\hline D. rerio & $--------1---g-p--g a------y g^{---g--n-----~}$ & 280 \\
\hline T. S. elegans & FDNWSTFRPRTSSNASTISGRLSPILTEQDDLGDGDVHSM & 167 \\
\hline G. gallus & 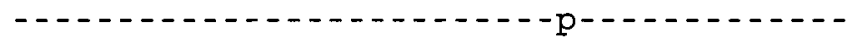 & 416 \\
\hline H. sapiens & $-------------------------m-------e------$ & 346 \\
\hline$X$. laevis & 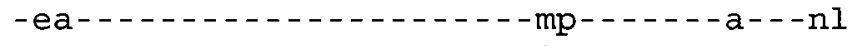 & 335 \\
\hline D. rerio & -ea-ta---------1-----fid.. -e---s-- mv & 318 \\
\hline T. s. elegans & VY . PSSATKMASTLPSLSULS . . . . . . NSENMENLLDNL & 199 \\
\hline G. gallus & $--.------t------8 m-\ldots \ldots$ s --------- & 448 \\
\hline H. sapiens & $--.-p--a----------d i-\ldots \ldots . p---------$ & 378 \\
\hline X. Iaevis & $--.----1 t-\ldots---$ femg. . . . . & 367 \\
\hline D. rerio & 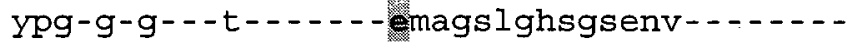 & 358 \\
\hline T. S. elegans & NLLSPSTSLTV . . . . . . . . . . . . . . . . . . & 210 \\
\hline gallus & $\cdots--n--m-\ldots$ stqsssaalmqqtpgysfasstts & 483 \\
\hline H. sapiens & $----s p----\ldots$ stqsspgtmmqqtpcysfappnts & 413 \\
\hline X. laevis & $---t-n s-\ldots \ldots \ldots$ tqsspasmmqqsgylftspnts & 397 \\
\hline D. rerio & --- - -knps-ggpgsgsnqsspsslmqaspgyspysspgl & 398 \\
\hline
\end{tabular}

FIG. 4.3. The partial amino acid sequence of FoxO1 from $T$. s. elegans compared with FoxO1 sequences from human (H. sapiens), chicken (G. gallus), western clawed frog (X. laevis), and zebrafish (D. rerio). Accession numbers are as above. Dashes replace amino acid residues identical with $T$. s. elegans whereas spacer dots indicate residues that are missing in the sequence. A turtle-specific amino acid substitution is bolded and shaded. 
FIG. 4.4

A)

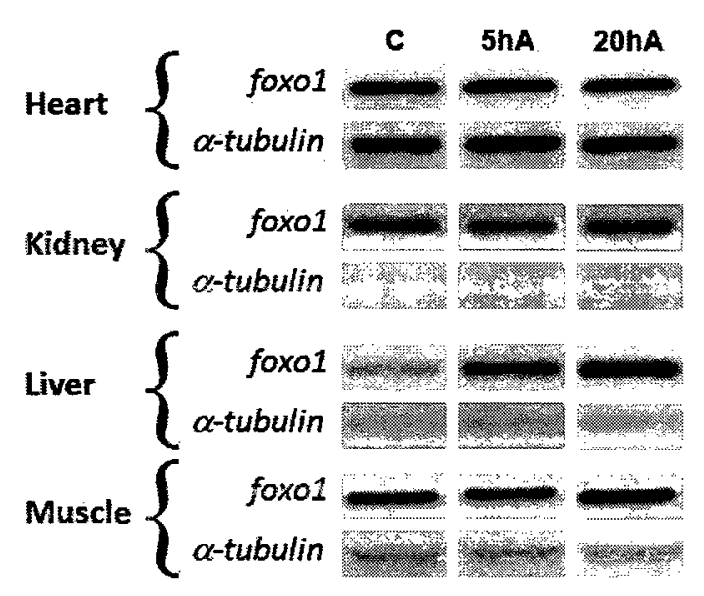

B)

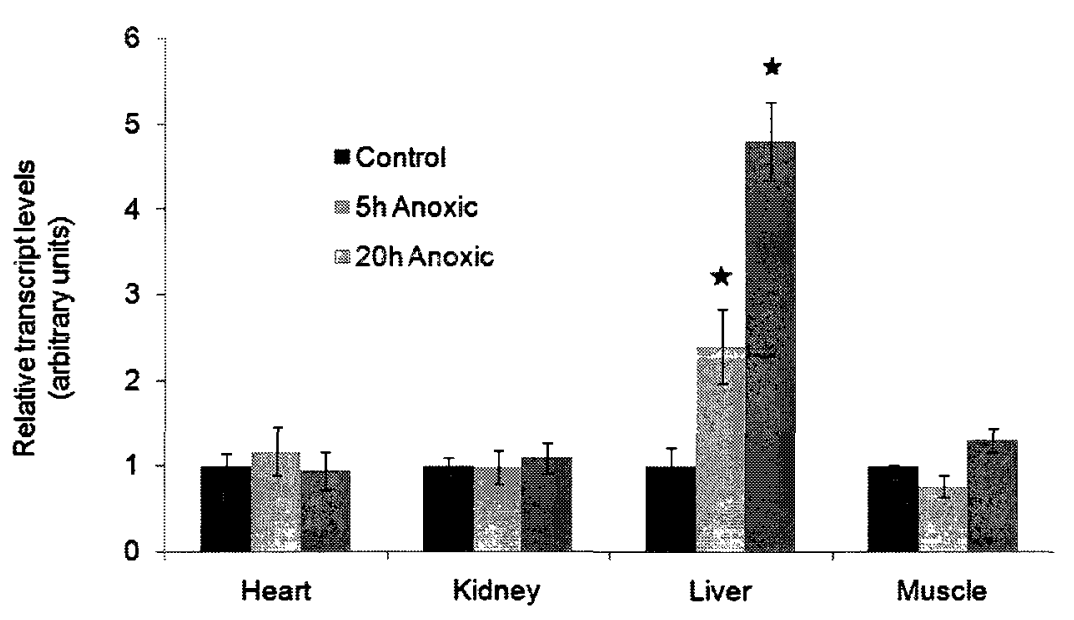

FIG. 4.4. Effect of 5 and $20 \mathrm{~h}$ anoxic submergence on foxol transcript expression in T. s. elegans tissues as determined by RT-PCR.

(A) Representative foxol amplicons with corresponding $\alpha$-tubulin bands under control and anoxic conditions. (B) Histogram showing normalized transcript levels under control and anoxic conditions; bands were normalized against the corresponding $\alpha$-tubulin bands from the same sample. Data are means \pm SEM, $n=3-4$ independent trials on mRNA isolated from different turtles. ${ }^{*}$ Significantly different from the corresponding control, $P$ $<0.05$. 
FIG. 4.5
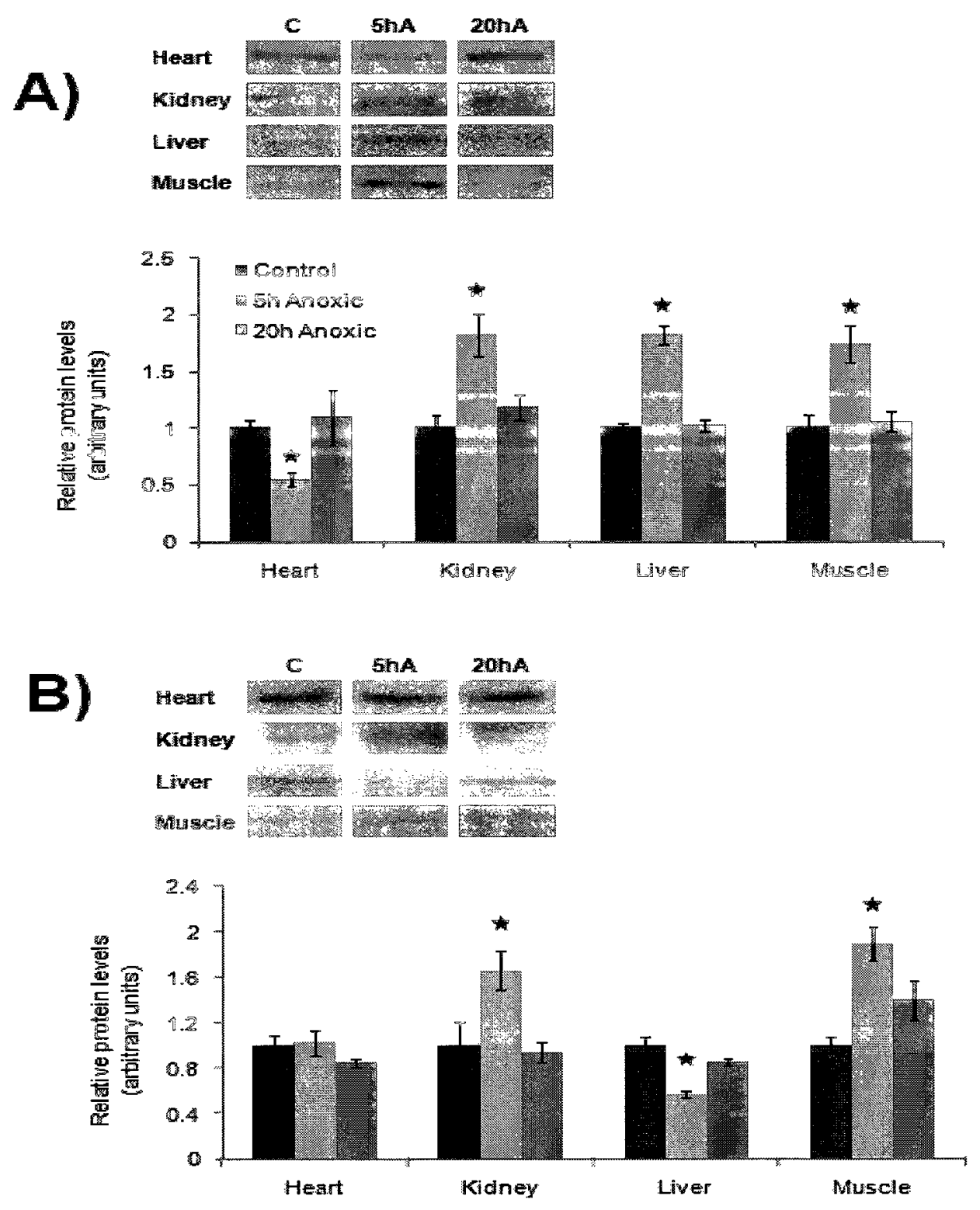

FIG. 4.5. Effect of 5 and $20 \mathrm{~h}$ anoxic submergence on the protein expression of FoxO1 and phospho-FoxO1 (Ser256) as determined by immunobloting.

(A) Representative western immunoblots showing changes in total FoxO1 levels in response to anoxia. The histogram shows normalized protein levels in turtle tissues. (B) Representative western immunoblots showing changes in p- FoxO1 (Ser 256) levels in response to anoxia. Histogram shows normalized protein levels in turtle tissues. Data are means \pm SEM, $n=3-5$ independent trials on tissue extracts from different turtles. * Significantly different from the corresponding control, $P<0.05$. 
FIG. 4.6

A)

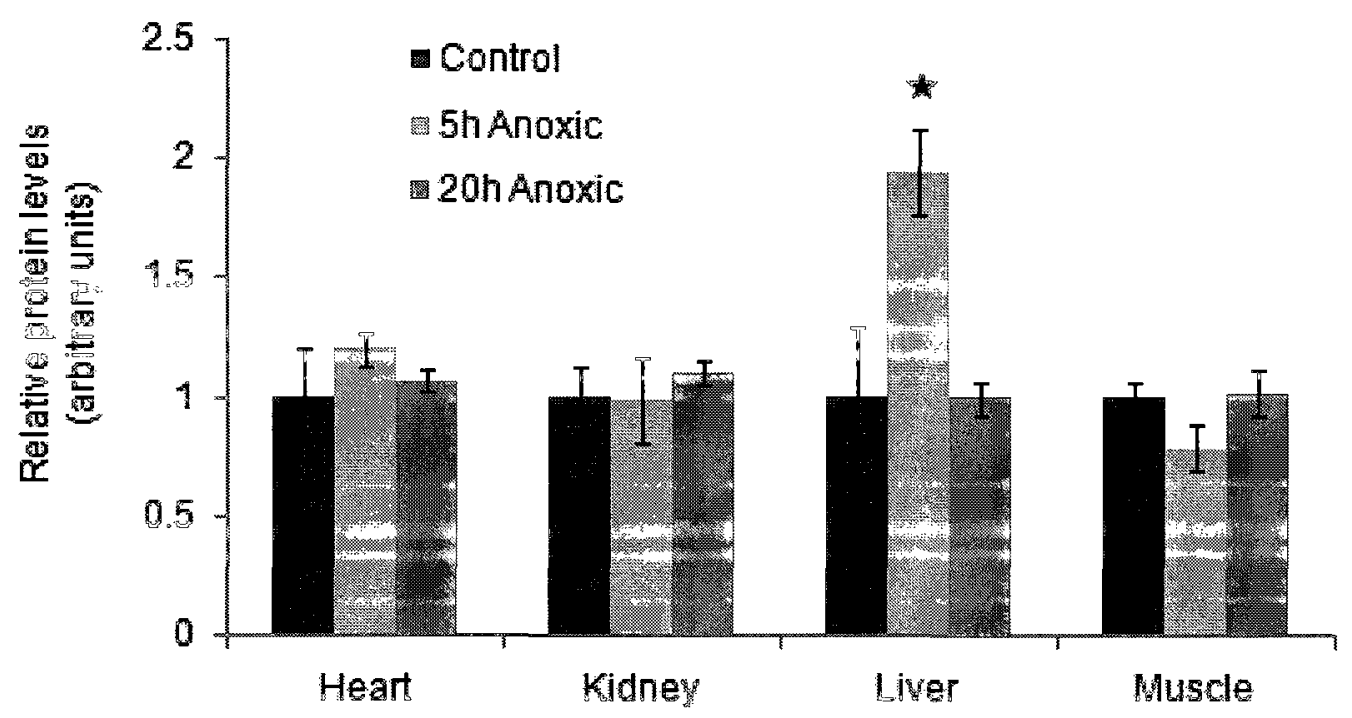

B)

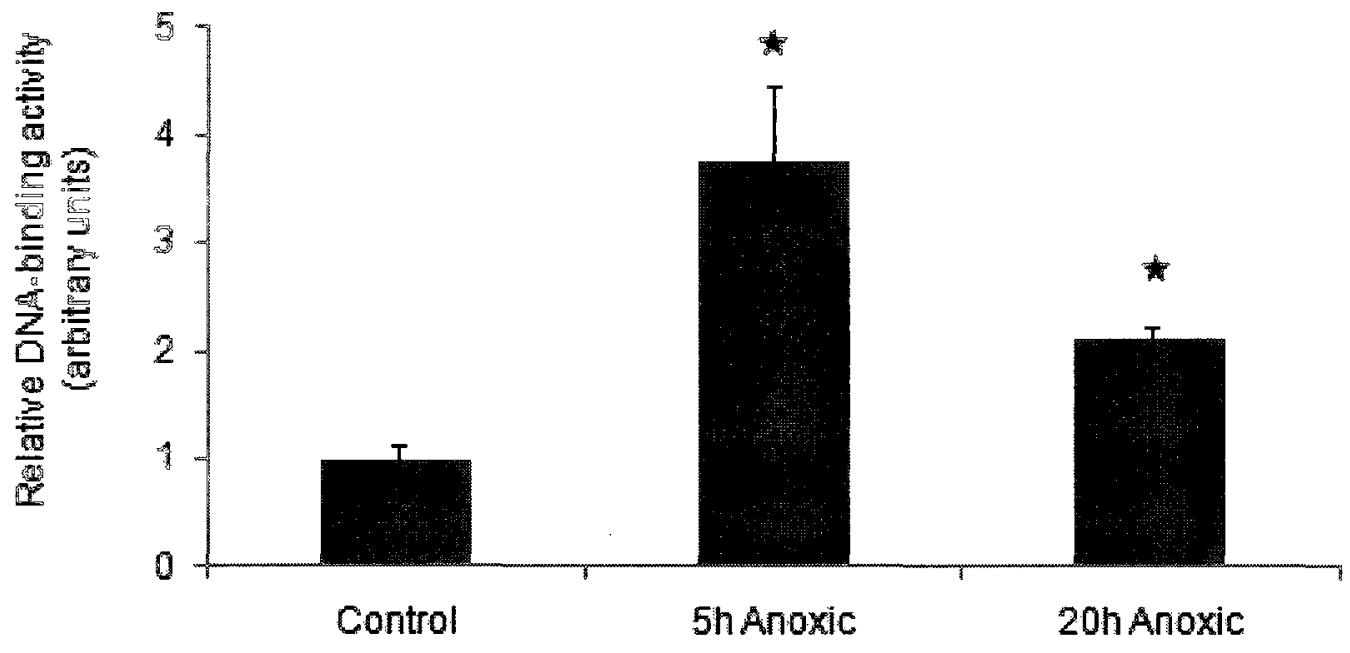

FIG. 4.6. Effect of 5 and $20 \mathrm{~h}$ anoxic submergence on FoxO1 activation in $T$. $s$. elegans tissues.

(A) Changes of FoxO1 protein levels in the nuclei of turtle tissues in response to anoxia, as determined by western immunoblotting.

(B) Changes in the DNA-binding activity of nuclear FoxO1 in the liver in response to anoxia, as determined by a transcription factor binding assay. Data are means \pm SEM, $n=3-4$ independent trials. * Significantly different from the corresponding control, $P<$ 0.05 . 
FIG. 4.7

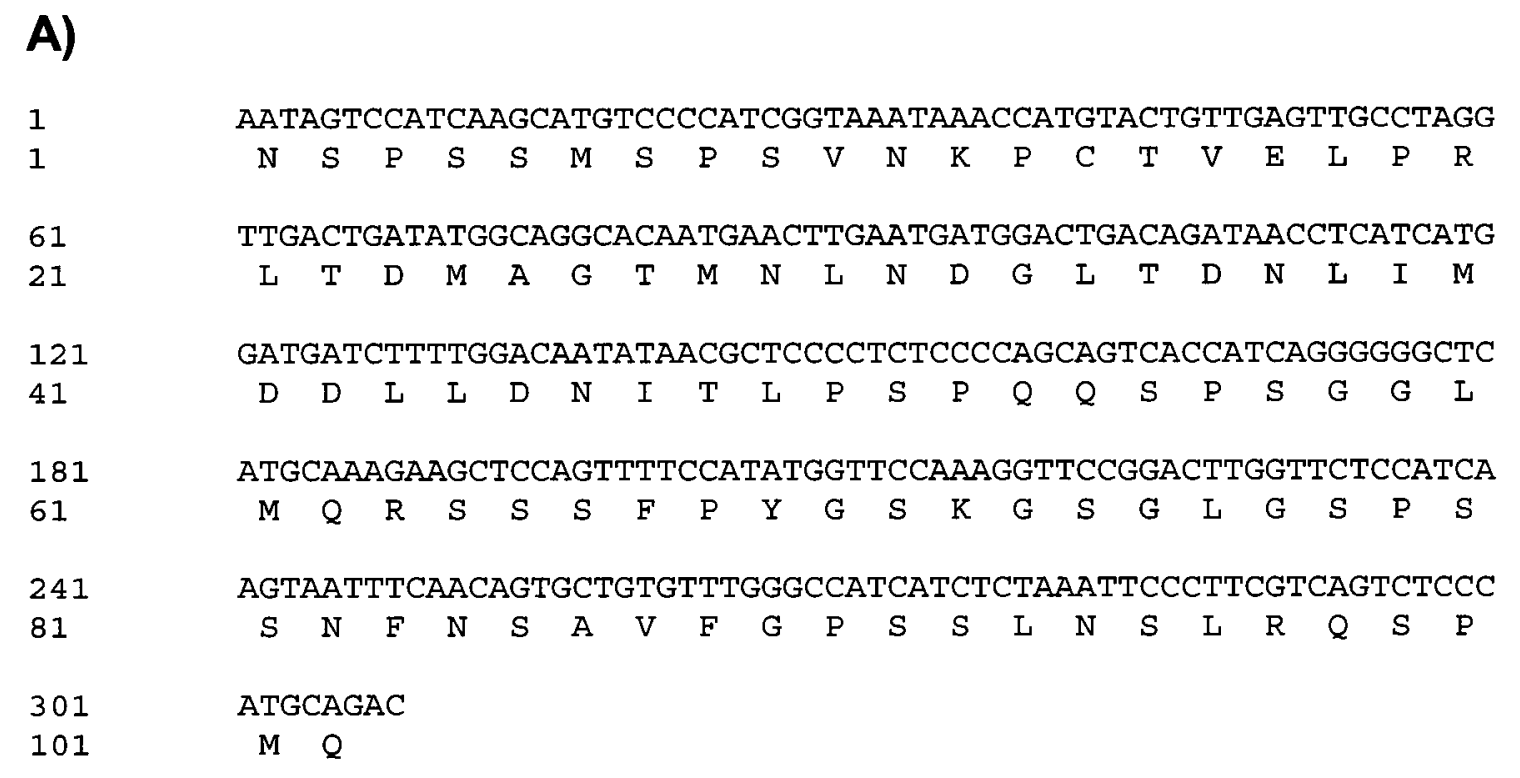

B)
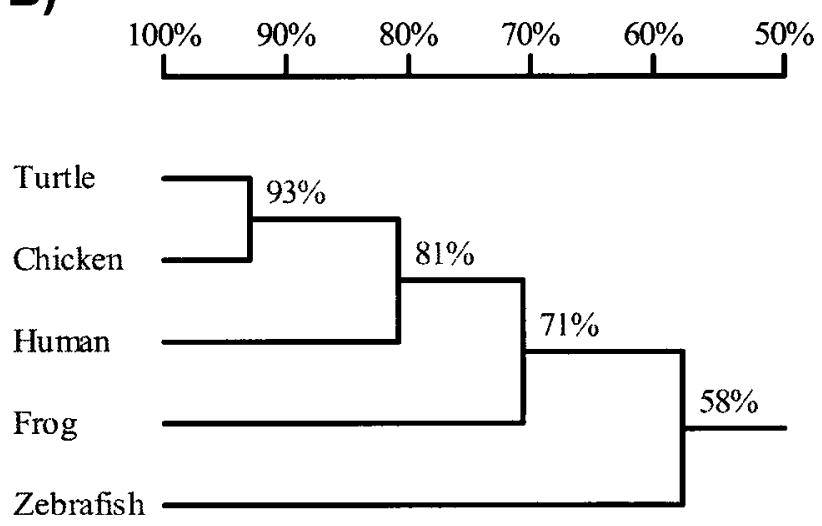

FIG. 4.7. Partial nucleotide and deduced amino acid sequence of $T$. s. elegans FoxO3.

(A) The partial cDNA sequence of foxo3, with the corresponding amino acid sequence is shown. Nucleotides and amino acids are numbered on the left. The nucleotide sequence was 308 nucleotides in length, and encoded 102 amino acids. This protein covered amino acids $389-480$ of the chicken FoxO3 sequence, representing $\sim 15 \%$ of the total FoxO3 protein.

(B) Homology tree produced from an alignment of the partial T. s. elegans FoxO3 protein sequence with FoxO3 from chicken (G. gallus), human (H. sapiens), African clawed frog (X. laevis) and zebrafish (D. rerio). Accession numbers are XP_001234496 for chicken, NP_001446 for human, NP_001086418 for frog and NP_001009988 for zebrafish. The percentage values correspond to the shared identity among the corresponding species. 
FIG. 4.8

\author{
T. s. elegans \\ G. gallus \\ H. sapiens \\ $X$. laevis \\ D. rerio \\ T. S. elegans \\ G. gallus \\ H. sapiens \\ $X$. laevis \\ D. rerio \\ T. s. elegans \\ G. gallus \\ $H$. sapiens \\ $X$. laevis \\ D. rerio
}

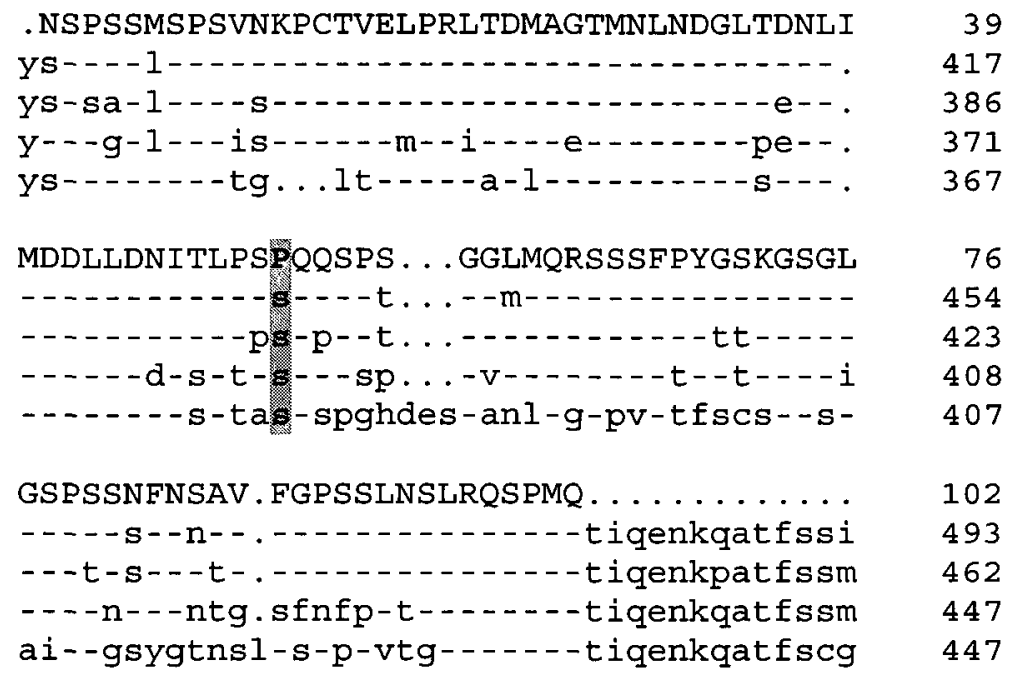

371

454

423

408

102

493

447

447

FIG. 4.8. The partial amino acid sequence of FoxO3 from $T$. s. elegans compared with FoxO3 sequences from human (H. sapiens), chicken (G. gallus), western clawed frog $(X$. tropicalis), and zebrafish (D. rerio). Accession numbers are as above. Dashes replace amino acid residues identical with $T$. s. elegans while spacer dots indicate residues that are missing in the sequence. A turtle-specific amino acid substitution is bolded and shaded. 
FIG. 4.9
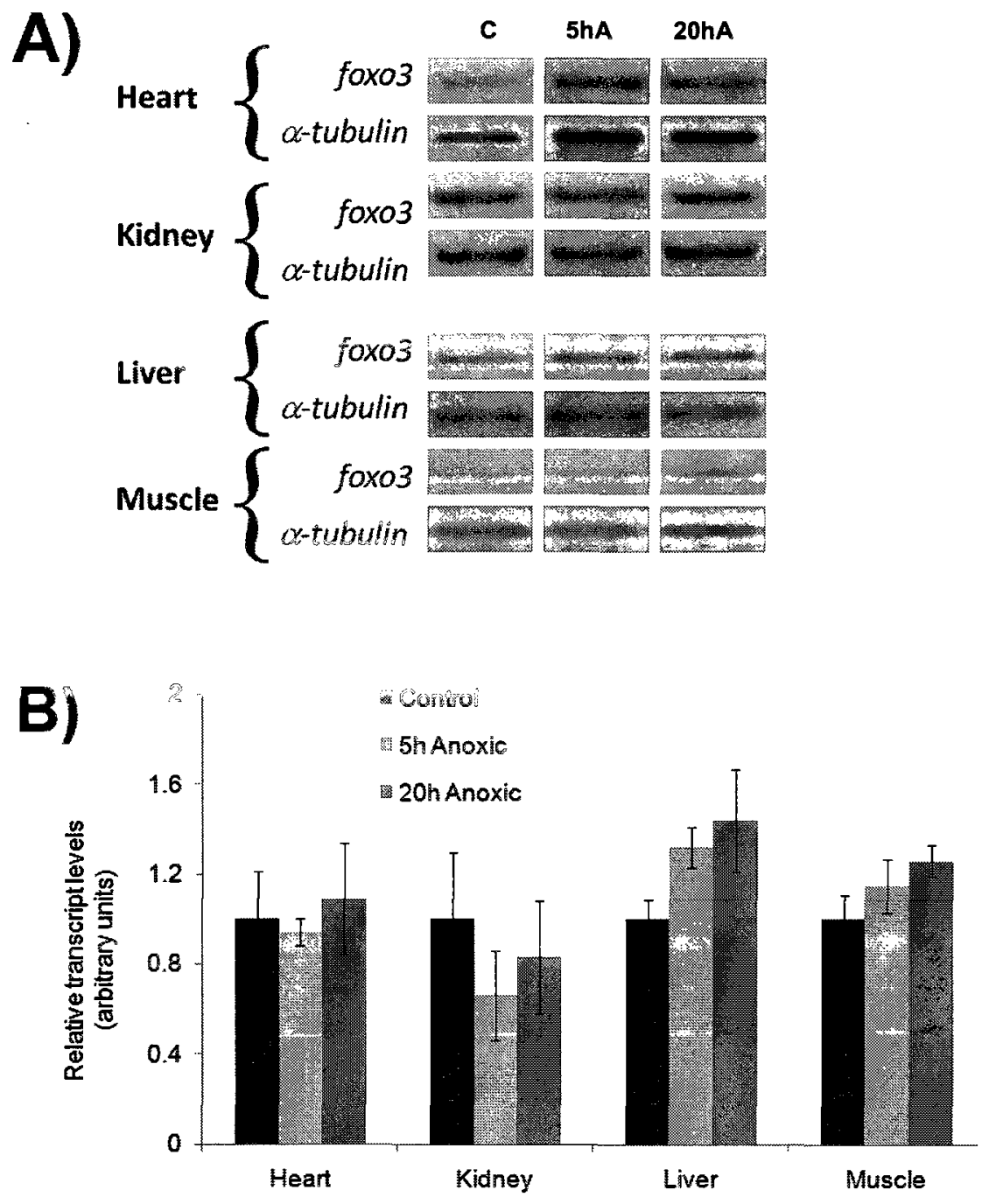

FIG. 4.9. Effect of 5 and $20 \mathrm{~h}$ anoxic submergence on foxo 3 transcript expression in T. s. elegans tissues as determined by RT-PCR.

(A) Representative foxo 3 amplicons with corresponding $\alpha$-tubulin bands under control and anoxic conditions.

(B) Histogram showing the normalized transcript levels under control and anoxic conditions; bands were normalized against the corresponding $\alpha$-tubulin bands from the same sample. Data are means $\pm \mathrm{SEM}, n=3-4$ independent trials on mRNA isolated from different turtles. ${ }^{*}$ Significantly different from the corresponding control, $P<0.05$. 
FIG. 4.10

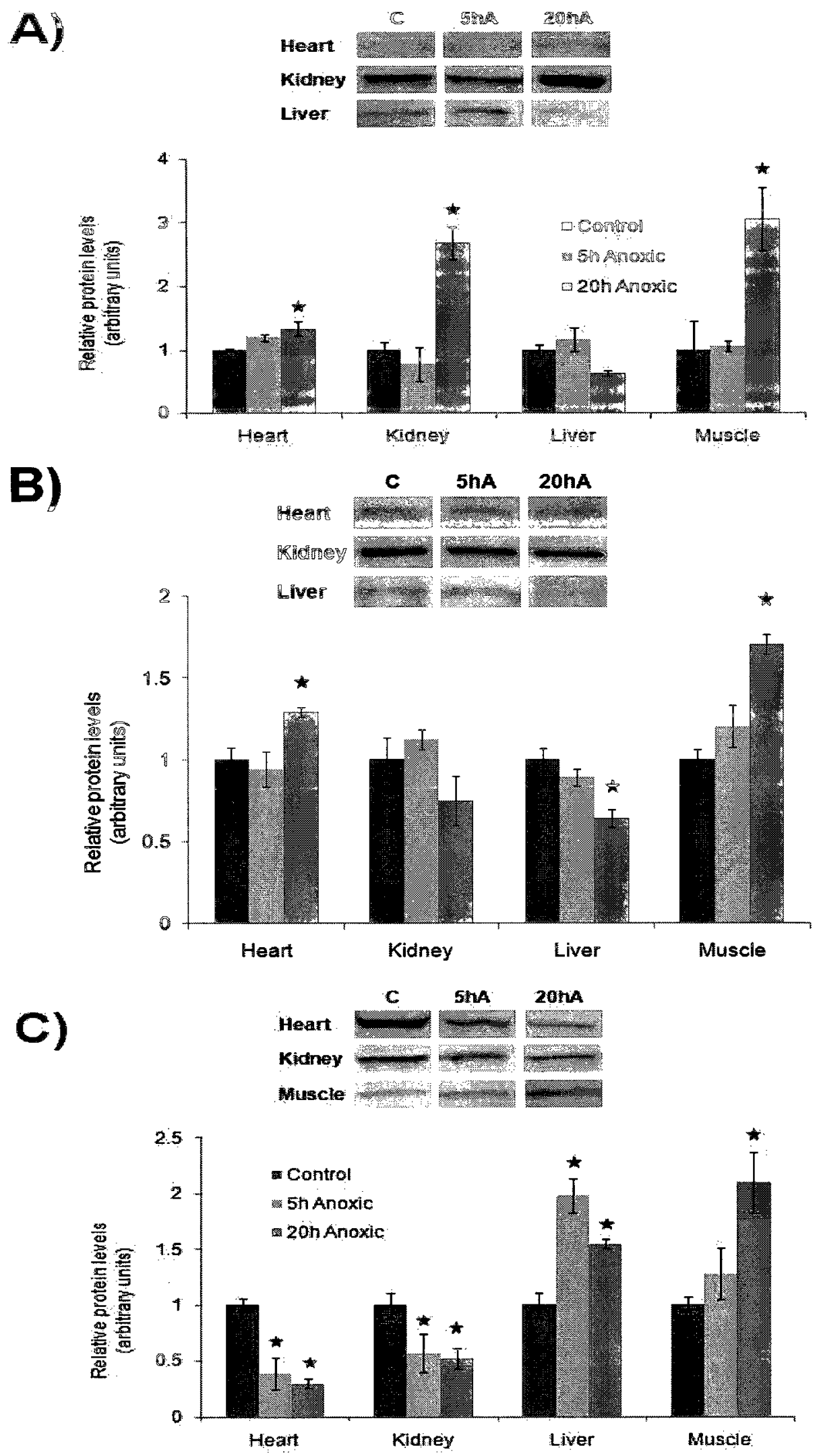


FIG. 4.10. Effect of 5 and $20 \mathrm{~h}$ anoxic submergence on the protein expression of FoxO3 and phospho-FoxO3 (Ser253 and Ser318/321) as determined by immunoblotting.

(A) Representative Western immunoblots showing changes in total FoxO3 levels in response to anoxia. Histogram shows normalized protein levels in turtle tissues.

(B) Representative Western immunoblots showing changes in p- FoxO3 (Ser 253) levels in response to anoxia. Histogram shows normalized protein levels in turtle tissues.

(C) Representative Western immunoblots showing changes in p- FoxO3 (Ser 318/21) levels in response to anoxia. Histogram shows normalized protein levels in turtle tissues.

Data are means \pm SEM, $n=3-5$ independent trials. * Significantly different from the corresponding control, $P<0.05$. 
FIG. 4.11
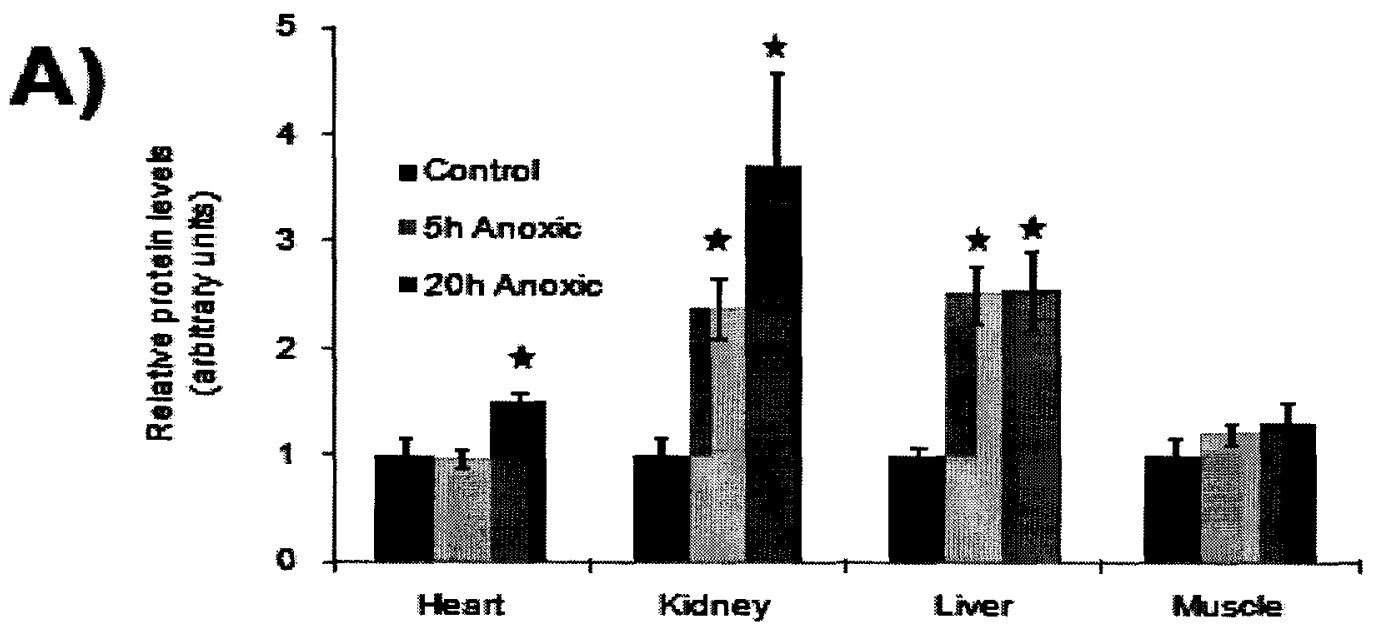

B)

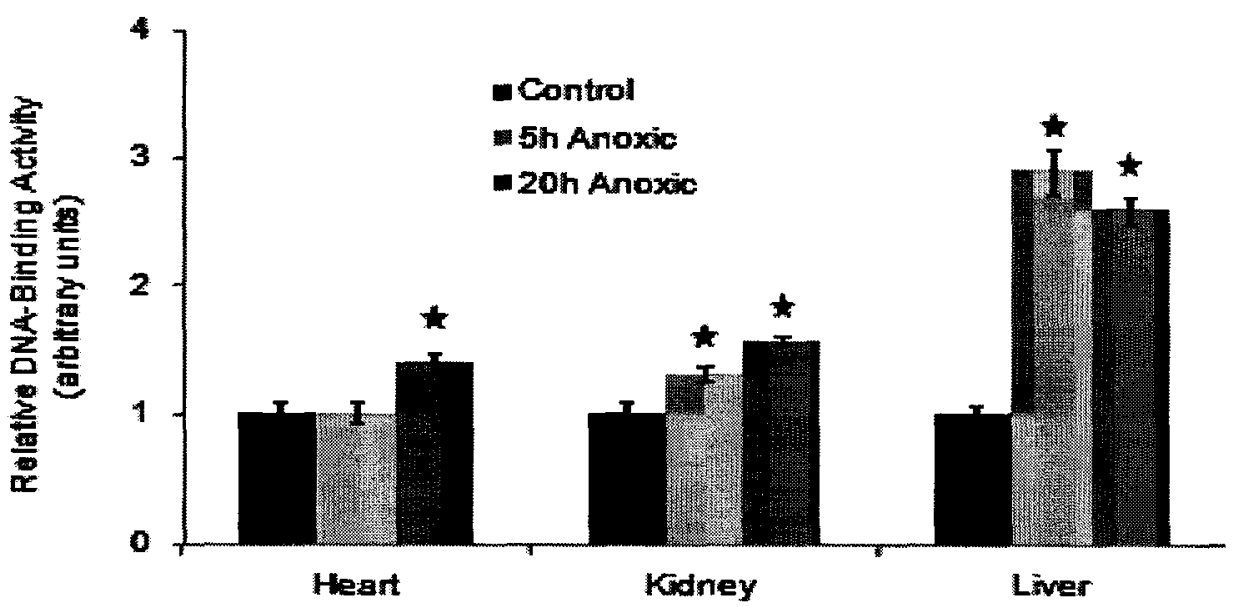

FIG. 4.11. Effect of 5 and $20 \mathrm{~h}$ anoxic submergence on FoxO3 activation in $T$. $s$. elegans tissues.

(A) Changes in FoxO3 protein levels in the nuclei of turtle tissues in response to anoxia, as determined by western immunoblotting.

(B) Changes in the DNA-binding activity of nuclear $\mathrm{FoxO} 3$ in response to anoxia, as determined by a transcription factor binding assay.

Data are means \pm SEM, $n=3-4$ independent trials for liver and muscle, and semiindependent trials for heart and kidney. ${ }^{*}$ Significantly different from the corresponding control, $P<0.05$. 


\section{FIG. 4.12}

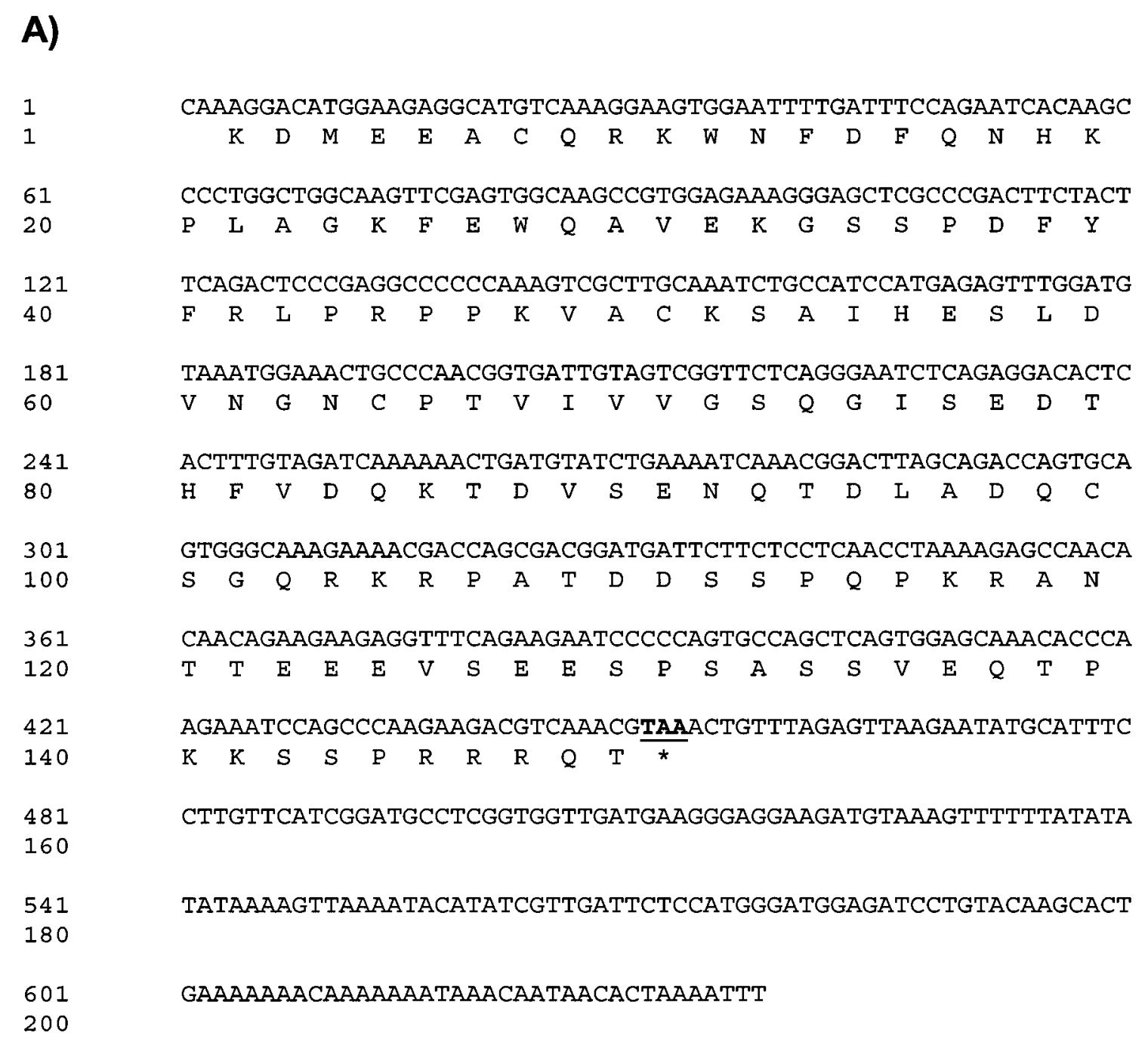

B) $\begin{array}{lllllll}100 \% & 90 \% & 80 \% & 70 \% & 60 \% & 50 \% & 40 \%\end{array}$

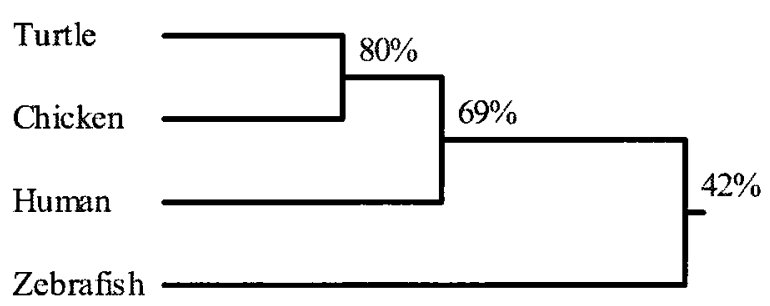


FIG. 4.12. Partial nucleotide and deduced amino acid sequence of $T$. s. elegans p27kip1.

(A) The partial cDNA sequence of $p 27 k i p 1$, with its corresponding amino acid sequence is shown. Nucleotides and amino acids are numbered on the left. The nucleotide sequence was 637 nucleotides in length and the stop codon is bolded and underlined. The sequence encoded 149 amino acids, covering amino acids 50-198 of the chicken p27kipl sequence, representing $\sim 75 \%$ of the total p27kipl protein and encompassing the entire Cterminus.

(B) Homology tree produced from an alignment of the partial T. s. elegans $\mathrm{p} 27 \mathrm{kip} 1$ protein sequence with $p 27 \mathrm{kipl}$ from chicken (G. gallus), human (H. sapiens) and zebrafish (D. rerio). Accession numbers are NP_989587 for chicken, NP_004055 for human and NP_997957 for zebrafish. The percentage values correspond to the shared identity among the corresponding species. 
FIG. 4.13

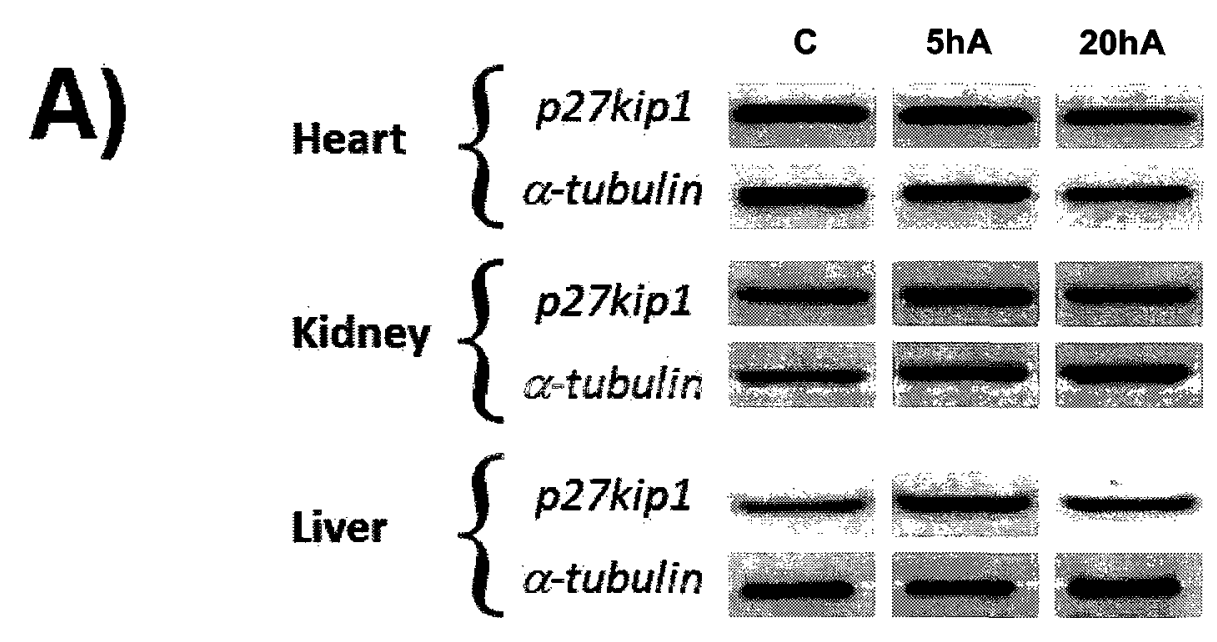

B)

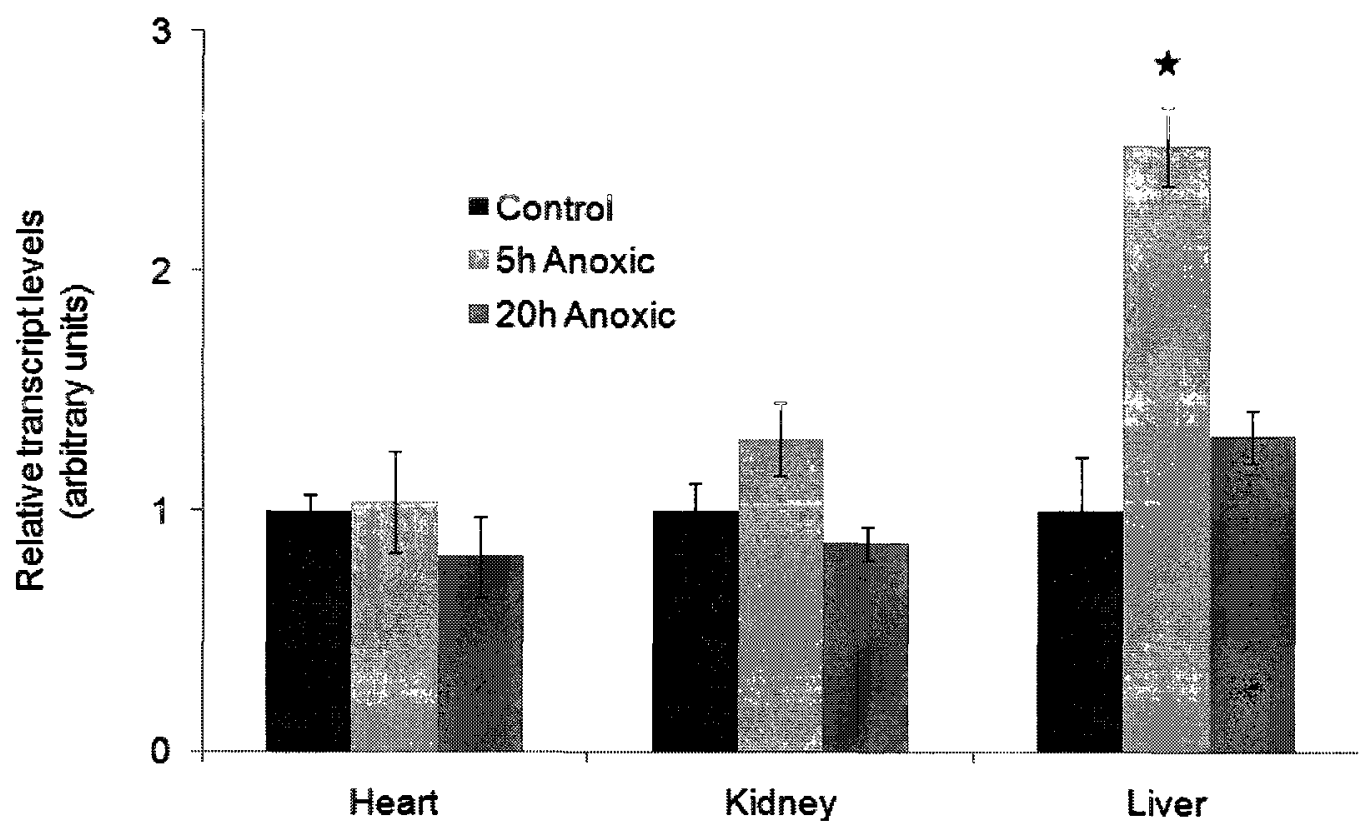

FIG. 4.13. Effect of 5 and $20 \mathrm{~h}$ anoxic submergence on p27kip1 transcript expression in $T$. s. elegans tissues as determined by RT-PCR.

(A) Representative $p 27 \mathrm{kipl}$ amplicons with corresponding $\alpha$-tubulin bands under control and anoxic conditions.

(B) Histogram showing the normalized transcript levels under control and anoxic conditions; bands were normalized against the corresponding $\alpha$-tubulin bands from the same sample. Data are means $\pm \mathrm{SEM}, n=3-4$ independent trials for mRNA isolated from different turtles. * Significantly different from the corresponding control, $P<0.05$. 
FIG. 4.14

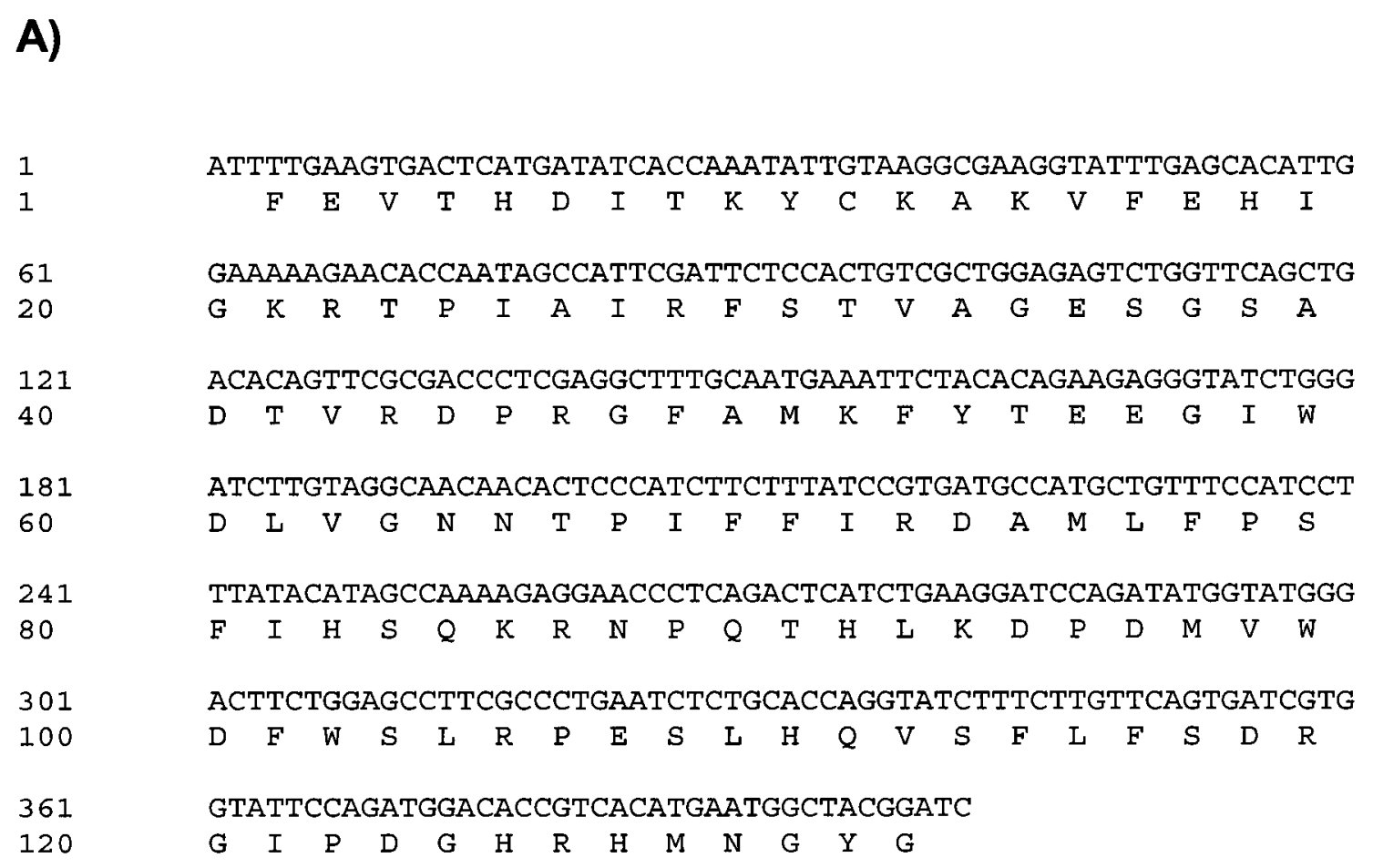

B)
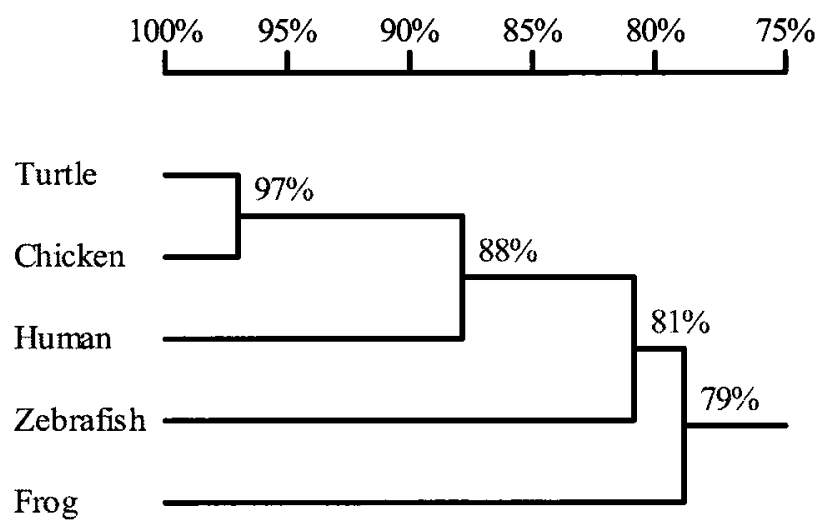
FIG. 4.14. Partial nucleotide and deduced amino acid sequence of $T$. s. elegans catalase.

(A) The partial cDNA sequence of catalase, with the corresponding amino acid sequence is shown. Nucleotides and amino acids are numbered on the left. The nucleotide sequence was 400 nucleotides in length, encoding 133 amino acids. This sequence covered amino acids $85-216$ of the chicken catalase sequence, representing $\sim 25 \%$ of the total catalase protein.

(B) Homology tree produced from an alignment of the partial T. s. elegans catalase protein sequence with catalase from chicken (G. gallus), human (H. sapiens) and zebrafish (D. rerio). Accession numbers are CAG31019 for chicken, NP_001743 for human, NP_001088663 for frog and NP_570987 for zebrafish. The percentage values correspond to the shared identity among the corresponding species. 
FIG. 4.15

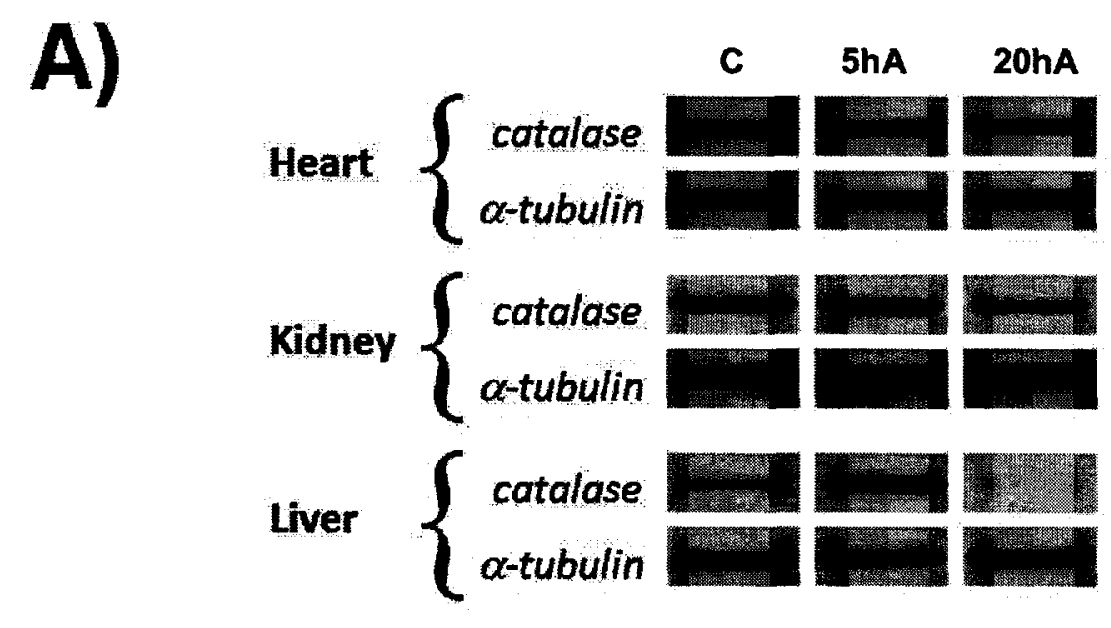

B)

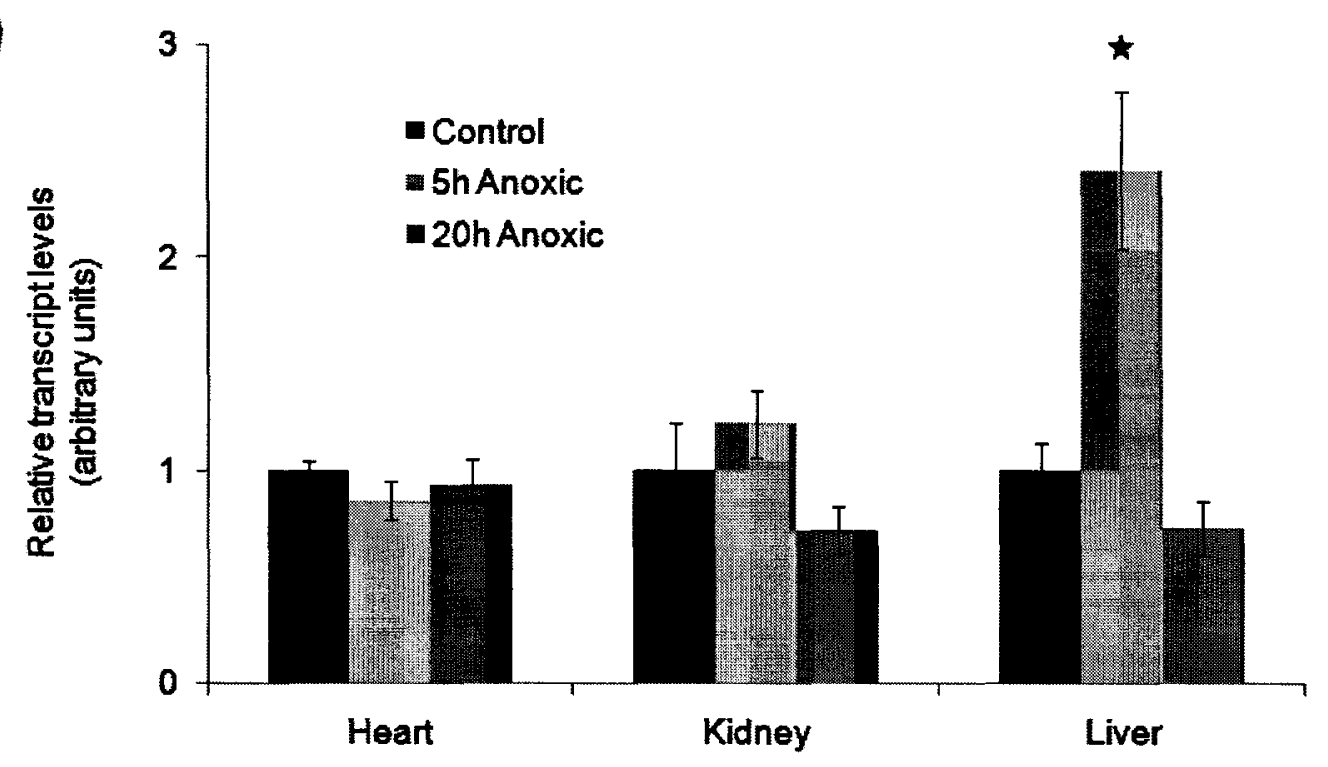

FIG. 4.15. Effect of 5 and $20 \mathrm{~h}$ anoxic submergence on catalase transcript expression in T. s. elegans tissues as determined by RT-PCR.

(A) Representative catalase amplicons with corresponding $\alpha$-tubulin bands under control and anoxic conditions.

(B) Histogram showing the normalized transcript levels under control and anoxic conditions; bands were normalized against the corresponding $\alpha$-tubulin bands from the same sample. Data are means \pm SEM, $n=3-4$ independent trials using mRNA isolated from different animals. * Significantly different from the corresponding control, $P<$ 0.05 . 


\section{CHAPTER 5}

\section{Epigenetics in anoxia tolerance: a role for histone deacetylases}

(Published in Mol. Cell. Biochem) 


\subsection{INTRODUCTION}

The term epigenetics refers to a variety of processes that lead to heritable changes in gene expression without a change in the DNA sequence (Wolffe and Matzke, 1999). These changes are orchestrated by modifications to DNA and chromatin proteins that result in altered gene transcription. Epigenetic modifications are of profound importance to cells and appear to play roles in many, if not all, biological processes throughout life. For example, epigenetic processes have been implicated in cell cycle control (Macaluso et al., 2005), disease (Barros and Offenbacher, 2009; van der Maarel, 2008), development (Shi and Wu, 2009) and aging (Calvanese et al., 2009; Ostojić et al., 2009). However, some areas of study in which epigenetics might play a key role have been understudied. The potential role of epigenetics in animal survival of hypoxic or anoxic conditions, for example, so far remaines unknown. Most vertebrates, and especially mammals, are very sensitive to oxygen deprivation and episodes of hypoxia or anoxia can be extremely damaging to tissues. Examination of the role of epigenetics in animal anoxia tolerance could improve our understanding of the molecular nature of how animals survive without oxygen. This, in turn, could shed light on the mechanisms underlying hypoxic injury in oxygen-sensitive cells.

Due to the importance of epigenetics in the regulation of many cellular processes, epigenetic modifications could potentially play a key role in the regulation of anoxiatolerance in turtle cells. Of particular value would be the use of epigenetic modifications to globally suppress gene transcription. Gene transcription is one of the major ATPconsuming processes in the cell, typically consuming $1-10 \%$ of the cell's energy budget (Rolfe and Brown, 1997). Regulation of gene transcription is accomplished by multiple 
mechanisms, providing controls over single genes, suites of genes, or global control of the whole genome. Epigenetic modification of chromatin proteins alters their interactions with DNA to increase/decrease the accessibility of DNA to the transcriptional apparatus. Histones, for example, are subject to a variety of post-translational modifications (e.g. methylation, acetylation, ubiquitylation, phosphorylation) that affect the state of transcriptional activity in the cell (Allfrey et al., 1964; Spotswood and Turner, 2002). In the case of histone $3(\mathrm{H} 3)$ in particular, it has been shown that posttranslational modifications are closely related to activation and repression of transcription. In general, acetylation of $\mathrm{H} 3$ leads to transcriptional activation whereas deacetylation causes transcriptional repression. Histone deacetylases (HDACs) are a group of enzymes that remove acetyl groups from histones and, therefore, are associated with transcriptional silencing (Reviewed by Burke and Baniahmad, 2002; Wade, 2001; Biel et al., 2005). HDAC activities can be affected by multiple signals/stresses including changes in oxygen levels, and HDACl was shown to be induced in response to low oxygen (Kim et al., 2001).

As mentioned previously, one of the major adaptations that confer anoxia tolerance in $T$. s. elegans is the ability to strongly depress metabolic rate to $10-20 \%$ of the corresponding aerobic rate at the same temperature. Metabolic depression is characterized by a reduction in the rates of most of the ATP-consuming processes in the cell including ion transport, protein synthesis and degradation, and transcription (Hochachka et al., 1996; Storey and Storey, 2004). The depression of their metabolic rate in response to anoxia allows turtles to minimize their metabolic demands, so that all cellular demands can be met by anaerobic glycolysis alone. Because of the important role 
of HDACs in suppressing transcription, I hypothesized that these proteins would become activated in response to anoxia in turtle tissues. The present chapter examines the expression of HDACs in three tissues, analyzing changes in Hdac transcript and HDAC protein levels in response to 5 and $20 \mathrm{~h}$ of submergence anoxia. Partial sequences for five turtle HDACs (HDAC1-5) were also obtained. In addition, HDAC activity in turtle muscle was measured under aerobic and anoxic conditions.

\subsection{MATERIALS AND METHODS}

\subsubsection{Animals}

All animal experiments were conducted as described in Chapter 2.

\subsubsection{Total RNA extraction and $c D N A$ synthesis}

RNA extraction and cDNA synthesis were performed as described in Chapter 2.

\subsubsection{Primers}

Forward and reverse primers for $H d a c 1-5$ were designed from conserved regions in these genes based on DNA alignment of the sequences from other vertebrate species as described in chapter 2. For Hdac2-4 perfect (species-specific) T. s .elegans primers to be used for quantification were designed after an initial sequence was obtained. Primers for 
the control gene, $\alpha$-tubulin, were as described in chapter 3 . The sequences of all the primers are listed in table 5.1.

\subsubsection{PCR amplification}

PCR amplification was performed as described in Chapter 2. The PCR program was as follows: $7 \mathrm{~min}$ at $94^{\circ} \mathrm{C}$ followed by 37 cycles of $1 \mathrm{~min}$ at $94^{\circ} \mathrm{C}, 1 \mathrm{~min}$ at a transcript-specific Ta of $50-62.5^{\circ} \mathrm{C}$, and $1.5 \mathrm{~min}$ at $72^{\circ} \mathrm{C}$. The final step was $72^{\circ} \mathrm{C}$ for 10 min. Ta values were $50^{\circ} \mathrm{C}$ for $H d a c 2$ and $H d a c 3$ (original primers), $54^{\circ} \mathrm{C}$ for $\alpha$ - tubulin, $H d a c 2$ and $H d a c 3$ (perfect primers), $H d a c 4$ (original primers) and $H d a c 5$, and $62.5^{\circ} \mathrm{C}$ for Hdacl and Hdac4 (perfect primers). PCR products were separated on $1 \%$ agarose gels stained with ethidium bromide, visualized using the ChemiGenius Bio-Imaging system (Syngene, Frederick, MD, USA) under UV light and quantified using the GeneTools program. Bands from the most dilute cDNA sample that gave visible product were used for quantification to ensure that the products did not reach amplification saturation.

\subsubsection{Sequencing}

The PCR products were sequenced by DNA Landmarks (St.-Jean-sur-Richelieu, QC). The sequences were verified as encoding the correct genes using the program BLASTN (http://www.ncbi.nlm.nih.gov/blast) at the NIH. The sequences were submitted to GenBank with the following accession numbers: GU063806, GU063807, GU063808, GU063809 and GU063810 for Hdac1-5, respectively. 


\subsubsection{Preparation of tissue extracts and Western Blotting}

Preparation of tissue extracts and western blotting were performed as described in Chapter 2. For western blotting, aliquots containing 30-40 $\mu \mathrm{g}$ protein from control and stress conditions were loaded into lanes of polyacrylamide gels (10-15\% gels depending on the protein being analyzed). Gels were run in a Mini Protean III apparatus (BioRad) at $180 \mathrm{~V}$ for $45 \mathrm{~min}$ at RT. The resolved proteins were transferred onto nitrocellulose membranes at $70 \mathrm{~V}$ for $90 \mathrm{~min}$ at $4^{\circ} \mathrm{C}$. Membranes were blocked for $15-30 \mathrm{~min}$ in Trisbuffered saline containing $0.05 \%$ Tween-20 (TBST) with 5\% milk. The blots were then probed overnight with primary antibodies for HDAC1, HDAC2, HDAC3, HDAC4, HDAC5, phosphorylated HDAC4 (Ser 632), phosphorylated HDAC5 (Ser 498), Histone H3 or acetyl histone H3 (Lys 9 or Lys 23). All antibodies were from Cell Signaling with the exception of p-HDAC4 and p-HDAC5 that were from GenScript. Antibodies were diluted 1:1000 in TBST. After incubation, blots were washed several times with TBST and then incubated at RT for 90 min with HRP-linked goat anti-rabbit IgG (Cell Signaling, diluted 1:2000 in TBST). Immunoblots were developed using enhanced chemiluminescence $(\mathrm{ECL})$ reagents.

\subsubsection{Preparation of nuclear extracts}

Nuclear extracts from all tissues were prepared as described in Chapter 2, with the exception that following quantification, the concentration of the extracts were adjusted to 5-8 to $8 \mu \mathrm{g} / \mu \mathrm{L}$ with extraction buffer without further manipulation. Extracts were used to assay for HDAC activity. 


\subsubsection{Histone deacetylase assay}

Histone deacetylase (HDAC) activity was assayed using the HDAC assay kit from Cayman Chemical (Ann Arbor, MI), according to the manufacturer's instructions. Briefly, paired samples were run where $10 \mu \mathrm{L}$ aliquots of each nuclear extract were combined with $150 \mu \mathrm{L}$ of HDAC assay buffer or $140 \mu \mathrm{L}$ of HDAC assay buffer $+10 \mu \mathrm{L}$ of Trichostatin A (a specific inhibitor of class I and class II HDACs), followed by addition of $10 \mu \mathrm{L}$ of HDAC substrate. The mixture was incubated for $30 \mathrm{~min}$ at $\mathrm{RT}$ and then developed by addition of $40 \mu \mathrm{L}$ of developer, followed by incubation for $15 \mathrm{~min}$ at RT. Fluorescence of samples was then measured in a microplate reader (excitation=350$380 \mathrm{~nm}$, emission=440-460 nm). HDAC-dependent fluorescence was calculated as the difference between total fluorescence and Trichostatin A inhibited fluorescence and then activity was expressed as relative fluorescence units per microgram protein.

\subsubsection{Data and statistics}

Data analysis was performed as described in Chapter 2.

\subsection{RESULTS}

\subsubsection{Cloning of partial Hdac sequences from $\underline{\text { T. s. elegans }}$}

Using RT-PCR and primers derived from the consensus regions of Hdacl-5 from other vertebrate sequences, PCR products were retrieved from the total RNA prepared 
from white skeletal muscle of T. s. elegans. The PCR products were $438 \mathrm{bp}, 597 \mathrm{bp}, 455$ bp, $242 \mathrm{bp}$, and $262 \mathrm{bp}$ for Hdac1-5, respectively. Each PCR product was confirmed as encoding its respective HDAC by BLAST, and each turtle sequence was submitted to GenBank with the following accession numbers: GU063806, GU063807, GU063808, GU063809 and GU063810, for Hdac1-5, respectively. FIGs.5.1-5 show the nucleotide sequence for each HDAC together with the corresponding amino acid sequence.

\subsubsection{Hdac transcript expression levels in response to anoxia in $\underline{\text { T. s. elegans }}$ heart}

Relative mRNA transcript levels of $\mathrm{Hdacl}-5$ were assessed in hearts of aerobic control, $5 \mathrm{~h}$ anoxic, and $20 \mathrm{~h}$ anoxic turtles (FIG.5.6). FIG.5.6A shows representative RT-PCR bands of $H d a c$ amplicons with corresponding $\alpha$-tubulin bands whereas histograms in FIG.5.6B show mean relative levels of $H d a c$ transcripts under the three conditions. Hdac5 mRNA levels increased significantly in response to anoxic submergence, rising by $2.1 \pm 0.5$ fold as compared with controls in response to $5 \mathrm{~h}$ anoxia $(\mathrm{P}<0.05)$ and remaining elevated at $2.3 \pm 0.1$ higher than controls $(\mathrm{P}<0.05)$ at $20 \mathrm{~h}$ of anoxia. Hdac1-4 transcripts showed no significant change in expression in the heart in response to anoxia.

\subsubsection{Protein expression of HDACs in response to anoxia in $\underline{T}$. s. elegans heart}

Western blotting was used to assess changes in expression of HDAC proteins in the hearts of aerobic, $5 \mathrm{~h}$ anoxic, and $20 \mathrm{~h}$ anoxic turtles. The polyclonal HDAC antibodies cross-reacted with bands at the expected molecular mass for each HDAC; 
these were $\sim 60 \mathrm{kDa}$ for HDAC1 and HDAC2, $\sim 50 \mathrm{kDa}$ for HDAC3, $140 \mathrm{kDa}$ for HDAC4, and $\sim 120 \mathrm{kDa}$ for HDAC5. The phosphorylated forms of HDAC4 and HDAC5 were also examined since the subcellular localization of these HDACs is known to be controlled by protein phosphorylation. FIG.5.7A shows changes in the expression of class I HDACs (HDAC1-3) in response to anoxia, whereas FIG.5.7B shows class II HDACs (HDAC4-5) and their phosphorylated forms, p-HDAC4 (Ser 632) and p-HDAC5 (Ser 498), in response to anoxia in the heart. The only significant change seen in the heart was an anoxia-responsive significant increase in HDAC5 protein content which rose by $3.3 \pm 0.7$ fold in response to $20 \mathrm{~h}$ of anoxia exposure $(\mathrm{P}<0.01)$.

\subsubsection{Transcript levels of Hdacs in response to anoxia in $\underline{T . s . \text { elegans liver }}$}

Relative transcript levels of $H d a c l-5$ were assessed in the livers of aerobic, $5 \mathrm{~h}$ anoxic, and $20 \mathrm{~h}$ anoxic turtles. FIG.5.8A shows representative RT-PCR bands of $H d a c$ amplicons with corresponding $\alpha$-tubulin bands under aerobic and anoxic conditions whereas FIG.5.8B shows a graphical representation of changes in the relative levels of $H d a c$ transcripts. $H d a c l$ and Hdac4 transcript levels increased significantly in response to $5 \mathrm{~h}$ of anoxia exposure, rising by $1.5 \pm 0.03$ and $2.9 \pm 0.01$ fold, respectively, as compared with corresponding controls $(\mathrm{P}<0.01)$. Levels of both returned to normoxic control values after $20 \mathrm{~h}$ of anoxia. Transcripts of other Hdacs were unchanged in response to anoxia. 


\subsubsection{Protein expression of HDACs in response to anoxia in $\underline{T}$. s. elegans liver}

FIG.5.9A shows changes in the protein expression of class I HDACs (HDAC1-3) in response to anoxia, whereas FIG.5.9B shows the class II HDACs (HDAC4-5) and their phosphorylated forms. HDAC1 levels increased significantly by $1.6 \pm 0.1$ fold in response to $5 \mathrm{~h}$ of anoxia $(\mathrm{P}<0.05)$ but returned to control values by $20 \mathrm{~h}$. HDAC2 content decreased significantly in anoxic liver, falling to $50 \pm 10 \%$ and $33 \pm 4 \%$ of control values $(\mathrm{P}<0.01)$ in response to 5 and $20 \mathrm{~h}$ of anoxia, respectively. HDAC3 levels were unchanged. Protein levels of both class II HDACs increased in response to $5 \mathrm{~h}$, but not $20 \mathrm{~h}$, of anoxia with HDAC4 increasing by $2.1 \pm 0.2$ fold $(\mathrm{P}<0.01)$ and HDAC5 increasing by $1.6 \pm 0.1$ fold $(\mathrm{P}<0.05)$ as compared with controls. The amount of phosphorylated HDAC5 decreased to $60 \pm 10 \%$ of the control value in response to $5 \mathrm{~h}$ anoxia $(\mathrm{P}<0.05)$ but rebounded by $20 \mathrm{~h}$ of anoxia.

\subsubsection{Transcript expression of Hdacs in response to anoxia in $\underline{T \text {. s. elegans muscle }}$}

Relative transcript levels of $H d a c 1-5$ were also assessed in the white skeletal muscle (neck contractile muscle) of aerobic, $5 \mathrm{~h}$ anoxic, and $20 \mathrm{~h}$ anoxic turtles. FIG.5.10A shows representative RT-PCR bands of $H d a c$ amplicons with corresponding $\alpha$-tubulin bands under aerobic and anoxic conditions whereas histograms in FIG.5.10B show changes in the relative levels of $H d a c$ transcripts. Muscle was the most responsive tissue with respect to $H d a c$ transcript expression, with transcript levels of every HDAC significantly increasing in response to anoxia. Hdac1 transcript levels increased by $2.4 \pm$ 0.4 fold in response to $5 \mathrm{~h}$ of anoxia $(\mathrm{P}<0.05)$ and rose further to $4.6 \pm 0.3$ fold of control values by $20 \mathrm{~h}$ of anoxia $(\mathrm{P}<0.01)$. Hdac 3 transcripts rose by $4.6 \pm 0.2$ fold after 
$5 \mathrm{~h}$ anoxia $(\mathrm{P}<0.01)$ and were $2.5 \pm 0.4$ fold above control values after $20 \mathrm{~h}$ anoxia $(\mathrm{P}<$ 0.01). Hdac5 transcript levels were also elevated at both time points being $1.8 \pm 0.2$ and $1.7 \pm 0.1$ fold higher than controls at $5 \mathrm{~h}$ and $20 \mathrm{~h}$ anoxia $(\mathrm{P}<0.05)$, respectively. Hdac2 and Hdac 4 transcripts were unchanged after $5 \mathrm{~h}$ anoxia, but increased by $2.9 \pm 0.4$ fold and $1.3 \pm 0.03$ fold after $20 \mathrm{~h}$ anoxia $(\mathrm{P}<0.05)$

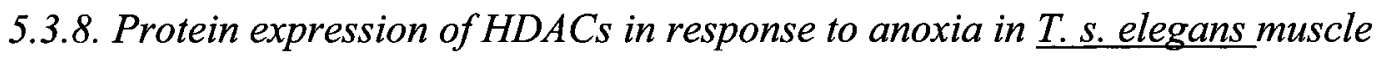

FIG.5.11A shows changes in the protein levels expression of class I HDACs (HDAC1-3) in response to anoxia, whereas FIG.5.11B shows the class II HDACs (HDAC4-5) and their phosphorylated forms. Muscle was also the most responsive tissue with respect to HDAC protein expression, with levels of every HDAC elevated significantly after $20 \mathrm{~h}$ anoxia and two also increased after $5 \mathrm{~h}$. HDAC1 levels rose by 2 \pm 0.2 fold in response to $20 \mathrm{~h}$ of anoxia $(\mathrm{P}<0.05)$ and HDAC2 rose by $1.7 \pm 0.2$ fold $(\mathrm{P}$ $<0.01)$. HDAC3 levels rose to $1.5 \pm 0.1$ fold of control in response to $5 \mathrm{~h}$ of anoxia $(\mathrm{P}<$ $0.05)$ and increased further to $2.3 \pm 0.2$ fold in response to $20 \mathrm{~h}$ anoxia $(\mathrm{P}<0.01)$. Total HDAC4 protein increased to $1.9 \pm 0.1$ fold greater than controls $(\mathrm{P}<0.01)$ in response to $20 \mathrm{~h}$ anoxia whereas HDAC5 protein levels were $3.7 \pm 0.3$ and $3.5 \pm 0.1$ fold higher than controls after 5 and $20 \mathrm{~h}$ of anoxia $(\mathrm{P}<0.01)$, respectively. Phosphorylated forms of class II HDACs were unchanged during anoxia. 


\subsubsection{HDAC activity in turtle muscle in response to anoxia}

HDAC activity is associated with transcriptional silencing. Since white skeletal muscle showed the greatest response by HDACs to anoxia, HDAC activities in this tissue were also measured. FIG.5.12 shows mean HDAC activity for the three treatment groups, expressed in relative fluorescence units per microgram soluble protein. Values were $49.2 \pm 4.6 \mathrm{RFU} / \mu \mathrm{g}$ in aerobic muscle, $44.3 \pm 3.8 \mathrm{RFU} / \mu \mathrm{g}$ after $5 \mathrm{~h}$ of anoxia, and 72 $\pm 7.3 \mathrm{RFU} / \mu \mathrm{g}$ after $20 \mathrm{~h}$ of anoxia exposure, representing a significant 1.5 -fold increase $(\mathrm{P}<0.05)$.

\subsubsection{Protein expression of total histone $H 3$ and acetyl histone $H 3$ in response to} anoxia

Polyclonal antibodies recognizing total histone $\mathrm{H} 3$ and its acetylated (Lys 9 and Lys23) forms were used to assess changes in histone acetylation in response to anoxia (FIG.5.13). All antibodies cross-reacted with a band at $\sim 17 \mathrm{kDa}$, the expected molecular weight of histone H3. FIG.5.13A shows changes in total histone H3 expression in response to anoxia in the three turtle tissues, whereas FIG.5.13B and FIG.5.13C show changes in the expression patterns of acetylated histone H3 Lys 9 and Lys 23, respectively. Total histone $\mathrm{H} 3$ levels did not change in heart or liver in response to anoxia, but levels rose in skeletal muscle by $2.2 \pm 0.2$ fold after $20 \mathrm{~h}$ of anoxia $(\mathrm{P}<0.01)$. Levels of acetyl-H3 (both Lys 9 and Lys 23) were unchanged in heart but decreased under anoxia in the other tissues. In liver acetyl-H3 (Lys 9) content dropped to $50 \pm 10 \%$ and $70 \pm 4 \%$ of control values after 5 and $20 \mathrm{~h}$ of anoxia $(\mathrm{P}<0.05)$. Similarly, acetyl-H3 (Lys 23) content dropped to $50 \pm 10 \%$ and $75 \pm 4 \%$ of control values after 5 and $20 \mathrm{~h}$ of 
anoxia $(\mathrm{P}<0.05)$. Levels of acetyl-H3 (Lys 9) in muscle decreased to $60 \pm 8 \%$ of control values after $5 \mathrm{~h}$ anoxia $(\mathrm{P}<0.01)$ and fell further to $40 \pm 5 \%$ of controls by $20 \mathrm{~h}$ of anoxia $(\mathrm{P}<0.01)$. Levels of acetyl-H3 (Lys 23 ) in muscle decreased to $60 \pm 7 \%$ of control after $5 \mathrm{~h}$ anoxia $(\mathrm{P}<0.01)$ and remained at $60 \pm 5 \%$ of control during $20 \mathrm{~h}$ of anoxia $(\mathrm{P}<0.01)$.

\subsection{DISCUSSION}

Metabolic rate depression (to about $10-20 \%$ of the corresponding aerobic rate at the same temperature) has been hailed as the most important contributing factor to anoxia survival. Metabolic depression is achieved by strong coordinated suppression and reprioritization of virtually all metabolic activities, particularly ATP-expensive processes in cells such as active transport, the cell cycle, protein translation, and gene transcription (Storey, 2007; Hochachka et al., 1996; Storey, and Storey, 2004). Multiple molecular mechanisms of metabolic rate depression in turtles have been elucidated, such as a prominent role of reversible protein phosphorylation and the importance of adenosine as a central nervous system depressant (reviewed by Storey, 2007; Lutz and Nilsson, 2004), but much still remains to be discovered.

In the present chapter, I focus on the regulation of gene transcription by epigenetics. In particular, I examined an important set of transcriptional regulators, the histone deacetylases (HDACs), to determine if there is a link between transcriptional silencing by HDACs and anoxia. The results show a tissue-specific upregulation pattern 
for HDACs in response to anoxia, suggesting these proteins play an important role in transcriptional silencing in $T$. s. elegans in response to anoxia. To my knowledge, this is the first study to examine these important transcriptional regulators in an anoxia-tolerant model.

Histone modifications are involved in the modulation of chromatin structure and play a key role in the regulation of gene transcription. Histone acetylation is a wellstudied modification that takes place at conserved lysine residues (Johnson and Turner, 1999). In particular, acetylation of histone $\mathrm{H} 3$ appears to have a dominant role in chromatin assembly (Strahl and Allis, 2000; Sobel et al., 1995; Chicoine et al., 1986). Actively transcribed chromatin regions have been linked with a higher acetylation state than those in condensed regions (heterochromatin), which are not accessible to the transcription machinery (Hebbes et al., 1988; Brown, 1966). Histone deacetylases (HDACs) are a family of enzymes involved in transcriptional repression through their ability to deacetylate histones. Known HDACs are grouped into different classes (class IIV) according to phylogenetic analyses and sequence homology to yeast protoypes. The present study examined several members from class I (HDAC1-3) and class II HDACs (HDAC4-5).

HDACs belonging to class I are homologous to yeast Rpd3 and include HDAC1, 2,3 , and 8 . These enzymes are found primarily in the nucleus and function as transcriptional repressors via deacetylation of histones. Since HDACs cannot interact directly with DNA or associated histones (Burke and Baniahmad, 2000; Wade, 2001), they must form complexes that allow them to bind to specific promoter regions (Biel et al., 2005). Class II HDACs possess catalytic domains with similarity to yeast Hdal and 
include HDAC4, 5, 6, 7, 9 and 10 . The catalytic activity of class II HDACs is fairly low (Lahm et al., 2007; Fischle et al., 2002; Jones et al., 2008) and their main function appears to be as co-repressors or adaptors through recruitment of other molecules. They have been shown to recruit class I HDACs through their C-terminal HDAC domain (Fischle et al., 2002) as well as interact with other transcriptional repressors, such as HP1 and C-terminal-binding protein (CTBP) (Zhang et al., 2001; Zhang et al., 2002b; Schuetz et al., 2008). In contrast to class I HDACs, class II HDACs are present in both nucleus and cytoplasm (Verdin et al., 2003; Fischle et al., 1999). The subcellular localization of class II HDACs is controlled by phosphorylation which promotes binding to 14-3-3 proteins and shuttling from the nucleus into the cytoplasm (Vega et al., 2004; McKinsey et al., 2000; Lu et al., 2000; Passier et al., 2000). This regulated phosphorylation provides a link between extracellular signals and transcriptional control. Whereas the main role of class I and II HDACs is in histone deacetylation, growing evidence suggests that virtually all HDACs have the ability to deacetylate non-histone proteins as well. This includes many important transcription factors such as NF-kB, STAT3 and p53, as well as other classes of proteins (reviewed by Glozak et al., 2005). This deacetylation activity is often inhibitory. As such, activation of HDACs can contribute to general transcriptional silencing not only through deacetylation of histones and chromatin condensation, but through inhibition of many cellular transcription factors.

Another important class of HDACs are the Sirtuins (or class III HDACs), that are involved in the deacetylation of a variety of protein targets in the cell (reviewed by Sauve et al., 2006). However, since the primary substrate of SIRTs is not histones, and their role 
in transcriptional repression in metazoan cells is not well-established, I did not examine these proteins in the present study.

Organ-specific responses by HDACs were observed in response to anoxia exposure in the turtle. In the white skeletal muscle a robust HDAC response was observed in response to $20 \mathrm{~h}$ of anoxia, with a uniform upregulation of all five HDACs occurring at both transcript (1.3-4.6 fold) and protein (by 1.7-3.5 fold) levels (FIG.5.10, 11). However, whereas both class II HDAC (HDAC4, HDAC5) protein levels increased in response to $20 \mathrm{~h}$ of anoxic submergence, the amount of phosphoprotein remained unchanged, which can be interpreted as a reduced percentage of phosphorylated HDAC4 and HDAC5; this is particularly true for HDAC5 which showed a $>3$-fold increase in protein content but no change in phosphorylation. A reduced percentage of phosphorylated HDAC4 and HDAC5 would suggest a predominantly nuclear localization during this time. This would be consistent with a repressional role for these HDACs under anoxic conditions. The strong increases in HDAC transcript and protein levels in response to $20 \mathrm{~h}$ of anoxia in skeletal muscle argue for a coordinated control of HDACs in response to anoxia exposure as a consequence of transcriptional activation of $H d a c$ genes. In order to determine whether the robust increase in HDAC protein levels in response to $20 \mathrm{~h}$ of anoxia also corresponds with increased activity, HDAC activity was assayed using a special kit. Unfortunately, this procedure does not allow the assay of specific HDACs and, as such, no kinetic data could be obtained. Instead, this assay provides data on the combined activity of class I and II HDACs, which was shown to be 1.5-fold higher in anoxic samples (FIG.5.12). Levels of acetylated histone H3 also decreased, dropping to $40 \%$ for AH3-lys 9 and $60 \%$ for AH3-Lys 23 (FIG.5.13B and C). 
Interestingly, a 2.2 -fold rise in the levels of histone $\mathrm{H} 3$ in response to $20 \mathrm{~h}$ of anoxia was also observed in muscle (FIG.5.13A). This increase could potentially address a need for increased compaction of chromatin during anoxia to impose global transcriptional repression. These results suggest that HDACs play a role in coordinating chromatin condensation in response to anoxia in white skeletal muscle and, therefore, may be key to anoxia-induced metabolic suppression in this tissue. Interestingly, while no other studies have reported HDAC up-regulation in response to anoxia in an anoxia-tolerant animal, activation of HDACs was shown to occur in the skeletal muscle of hibernating ground squirrels (Morin and Storey, 2006), which also undergo profound metabolic depression. Upregulation of the transcript levels of several epigenetic modifiers was also reported in skeletal muscle tissue of estivating desert frogs (Hudson et al., 2008). This suggests that activation of epigenetic mechanisms in turtle muscle might not be an anoxia-specific response, but part of a common mechanism of metabolic suppression across phylogeny.

Whereas skeletal muscle displayed a robust upregulation of HDACs in response to anoxia, the liver showed a milder response. Both transcript and protein levels of HDAC1 and HDAC4 increased in response to $5 \mathrm{~h}$ of anoxia, rising to 1.5-2.9 and 1.6-2.1 fold of control values, respectively, and HDAC5 also showed a 1.6-fold increase in protein levels (FIG.5.8-9). Phosphorylated HDAC5 content also decreased to $60 \%$ of control values (FIG.5.9B), which would imply nuclear retention of the enzyme. These data suggest a role for HDAC1, HDAC4 and HDAC5 in transcriptional suppression in liver in response to $5 \mathrm{~h}$ of anoxia. All of these HDACs returned to normoxic control levels when anoxia was prolonged to $20 \mathrm{~h}$. Levels of acetylated histone $\mathrm{H} 3$ also decreased more in response to $5 \mathrm{~h}$ than $20 \mathrm{~h}$ of anoxia , decreasing to $50 \%$ compared to 
$70 \%$ of control for Lys 9 and $50 \%$ compared to $75 \%$ for Lys 23 ( FIG.5.13). The milder HDAC response observed in the liver suggests that rather than promoting an overall state of higher chromatin condensation, liver HDACs, particularly those belonging to class II, play a role in silencing key promoter regions in response to short-term anoxia. It should also be noted that HDAC1 is known to be involved in cell cycle arrest through its interaction with the retinoblastoma protein $\mathrm{Rb}$ and the transcription factor $\mathrm{E} 2 \mathrm{~F}$ [Brehm et al., 1998]. Therefore, upregulation of $\mathrm{HDACl} 1$ in response to $5 \mathrm{~h}$ of anoxia could be involved in cell cycle arrest in turtle hepatocytes. The differences in the HDAC responses between skeletal muscle and liver might also be due to the functional differences in these tissues.

The heart showed the fewest responses to anoxia by the HDAC system. Only the class II HDAC, HDAC5, showed increased transcript (2.1-2.3 fold) and protein (3.3-fold after $20 \mathrm{~h}$ anoxia) expression (FIG.5.6-7). The phosphorylation state of this protein remained unchanged in response to anoxia, suggesting that this protein would remain primarily localized in the nucleus and exert a repressional role, possibly through recruitment of other repressive molecules. Overall, however, the heart displayed a lesser response by HDACs than did either of the other tissues, suggesting that HDACs (with the exception of HDAC5) do not play a major role in transcriptional silencing in the heart in response to anoxia. This conclusion is corroborated by the data for acetylated histone H3 levels, which did not change significantly in the heart in response to anoxia (FIG.5.13). These findings could also be explained by the functional differences between the heart and the other tissues. Heart remains active throughout an anoxic episode, since the pumping of blood is crucial for the survival of all organs providing both nutrient delivery 
and removal of wastes. As a result, it is possible that transcriptional silencing in the heart is not as prominent as it is in the liver or muscle, or that such silencing occurs by mechanisms other than chromatin condensation.

In conclusion, the present study suggests a role for epigenetics in anoxia tolerance. The data show up-regulation of HDACs in a tissue-specific manner in response to anoxia in T. s. elegans, with skeletal muscle showing the most robust response, suggesting that modulation of muscle chromatin structure by histone deacetylation is a physiologically relevant molecular mechanism of anaerobic survival. To better understand the role of epigenetics in anoxia tolerance, it might be of benefit for future studies to examine the effects of anoxia on other known mechanisms of transcriptional modulation, such as other proteins involved in modifications of histones or DNA. 
TABLE 5.1. List of Primers used in chapter 5.

\begin{tabular}{|c|c|c|c|}
\hline Gene/primer & Primer sequence (5'-3') & $\begin{array}{c}\text { Annealing } \\
\text { temp. } \\
\left({ }^{\circ} \mathrm{C}\right) \\
\end{array}$ & $\begin{array}{c}\text { Expected } \\
\text { product size } \\
\text { (bp) } \\
\end{array}$ \\
\hline Hdacl forward & GGAGAGTACTTCCCAGGAAC & \multirow[t]{2}{*}{62.5} & \multirow[t]{2}{*}{$\sim 500$} \\
\hline Hdacl reverse & AGAGACGCTGCTTGATCTTC & & \\
\hline $\begin{array}{l}\text { Hdac2 original } \\
\text { forward }\end{array}$ & TTGTGCTTGCCATCCTTGA & \multirow[t]{2}{*}{50} & \multirow[t]{2}{*}{$\sim 700$} \\
\hline $\begin{array}{l}\text { Hdac2 original } \\
\text { reverse }\end{array}$ & ACACCAGGTGCATGAGGTA & & \\
\hline $\begin{array}{l}\text { Hdac2 perfect } \\
\text { forward }\end{array}$ & CCAGTGCCGTGGTATTGCAG & \multirow[t]{2}{*}{54} & \multirow[t]{2}{*}{$\sim 400$} \\
\hline $\begin{array}{l}\text { Hdac2 perfect } \\
\text { reverse }\end{array}$ & CACCAGGTGCATGAGGTAAC & & \\
\hline $\begin{array}{l}\text { Hdac3 original } \\
\text { forward }\end{array}$ & TATGAAGTIGGIGCAGAGAG & \multirow[t]{2}{*}{50} & \multirow[t]{2}{*}{$\sim 500$} \\
\hline $\begin{array}{l}\text { Hdac3 original } \\
\text { reverse }\end{array}$ & GGTGCATGGTTCARCATCTT & & \\
\hline $\begin{array}{l}\text { Hdac3 perfect } \\
\text { forward }\end{array}$ & AACACCTCTTCCAGCCAGTC & \multirow[t]{2}{*}{54} & \multirow[t]{2}{*}{$\sim 400$} \\
\hline $\begin{array}{l}\text { Hdac3 perfect } \\
\text { reverse }\end{array}$ & TGGTGCATGGTTCAGCATCT & & \\
\hline $\begin{array}{l}\text { Hdac4 original } \\
\text { forward }\end{array}$ & GAGCAGGAGCTGCTCTTC & \multirow[t]{2}{*}{54} & \multirow[t]{2}{*}{$\sim 311$} \\
\hline $\begin{array}{l}\text { Hdac4 original } \\
\text { reverse }\end{array}$ & CGGGACCAGATGCTCTGGAT & & \\
\hline $\begin{array}{l}\text { Hdac4 perfect } \\
\text { forward }\end{array}$ & CGCATTCACCAGCTGAGAA & \multirow[t]{2}{*}{62.5} & \multirow[t]{2}{*}{$\sim 250$} \\
\hline $\begin{array}{l}\text { Hdac4 perfect } \\
\text { reverse }\end{array}$ & GGGACCAGATGCTCTGGAT & & \\
\hline Hdac5 forward & CAGAGCATCTGGTCCCG & \multirow[t]{2}{*}{54} & \multirow[t]{2}{*}{$\sim 400$} \\
\hline Hdac5 reverse & GAGTTGAAGAAGCAGAA & & \\
\hline
\end{tabular}


FIG.5.1

1

1

61

21

121

41

181

61

241

81

301

101

361

121

421

141
GTATTATGCTGTCAACTATCCTCTCCGGGACGGAATTGACGATGAATCCTATGAGGCAAT

$\begin{array}{llllllllllllllllllll}Y & Y & A & V & N & Y & P & L & R & D & G & I & D & D & E & S & Y & E & A & I\end{array}$

ATTTAAGCCGGTGATATCTAAAGTGATGGAGACGTTCCAGCCTAGTGCAGTTGCCTTACA

$\begin{array}{llllllllllllllllllll}F & K & P & V & I & S & K & V & M & E & T & F & Q & P & S & A & V & A & L & Q\end{array}$

GTGTGGATCGGATTCTCTGTCAGGGGACAGATTGGGCTGTTTTAATCTGACCATCAAAGG

$\begin{array}{lllllllllllllllllllll}C & G & S & D & S & L & S & G & D & R & L & G & C & F & N & L & T & I & K & G\end{array}$

TCATGCCAAGTGTGTGGAGTTCGTAAAGAGTTTTAACTTGCCTATGCTGATGCTAGGAGG

$\begin{array}{llllllllllllllllllll}\mathrm{H} & \mathrm{A} & \mathrm{K} & \mathrm{C} & \mathrm{V} & \mathrm{E} & \mathrm{F} & \mathrm{V} & \mathrm{K} & \mathrm{S} & \mathrm{F} & \mathrm{N} & \mathrm{L} & \mathrm{P} & \mathrm{M} & \mathrm{L} & \mathrm{M} & \mathrm{L} & \mathrm{G} & \mathrm{G}\end{array}$

GGGTGGCTACACAATCCGCAACGTGGCTAGATGCTGGACTTATGAGACTGCTGTGGCTTT

$\begin{array}{llllllllllllllllllll}G & G & Y & T & I & R & N & V & A & R & C & W & T & Y & E & T & A & V & A & L\end{array}$

GGACACGGAAATTCCAAATGAGCTTCCATATAATGACTATTTTGAATACTTTGGACCAGA

$\begin{array}{llllllllllllllllllll}\mathrm{D} & \mathrm{T} & \mathrm{E} & \mathrm{I} & \mathrm{P} & \mathrm{N} & \mathrm{E} & \mathrm{L} & \mathrm{P} & \mathrm{Y} & \mathrm{N} & \mathrm{D} & \mathrm{Y} & \mathrm{F} & \mathrm{E} & \mathrm{Y} & \mathrm{F} & \mathrm{G} & \mathrm{P} & \mathrm{D}\end{array}$

CTTTAAGCTCCACATCAGTCCTTCGAACATGACCAACCAGAACACCAATGAGTATCTTGA

$\begin{array}{llllllllllllllllllll}\mathrm{F} & \mathrm{K} & \mathrm{L} & \mathrm{H} & \mathrm{I} & \mathrm{S} & \mathrm{P} & \mathrm{S} & \mathrm{N} & \mathrm{M} & \mathrm{T} & \mathrm{N} & \mathrm{Q} & \mathrm{N} & \mathrm{T} & \mathrm{N} & \mathrm{E} & \mathrm{Y} & \mathrm{L} & \mathrm{E}\end{array}$

GAAGATCAAGCAGCGTCT

$\begin{array}{lllll}K & I & K & Q & R\end{array}$

FIG.5.1. Nucleotide and deduced amino acid sequence for T. s. elegans partial Hdacl sequence. Nucleotides and amino acids are numbered on the left. The nucleotide sequence was 439 nucleotides in length, while the amino acid sequence was 146 amino acids in length. 


\section{FIG.5.2}

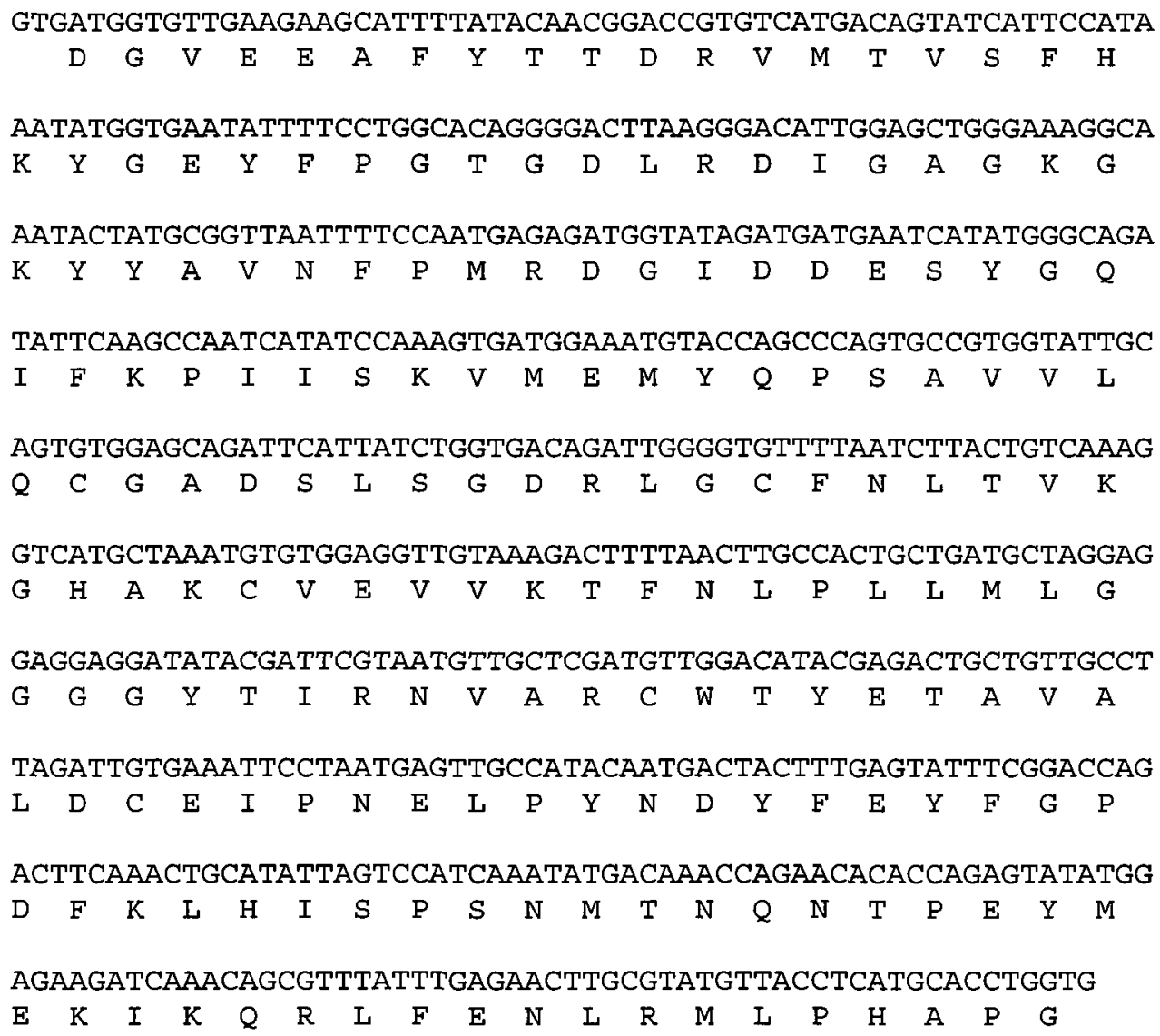

FIG.5.2. Nucleotide and deduced amino acid sequence for T. s. elegans partial Hdac2 sequence. Nucleotides and amino acids are numbered on the left. The nucleotide sequence was 599 nucleotides in length, while the amino acid sequence was 199 amino acids in length. 


\section{FIG.5.3}

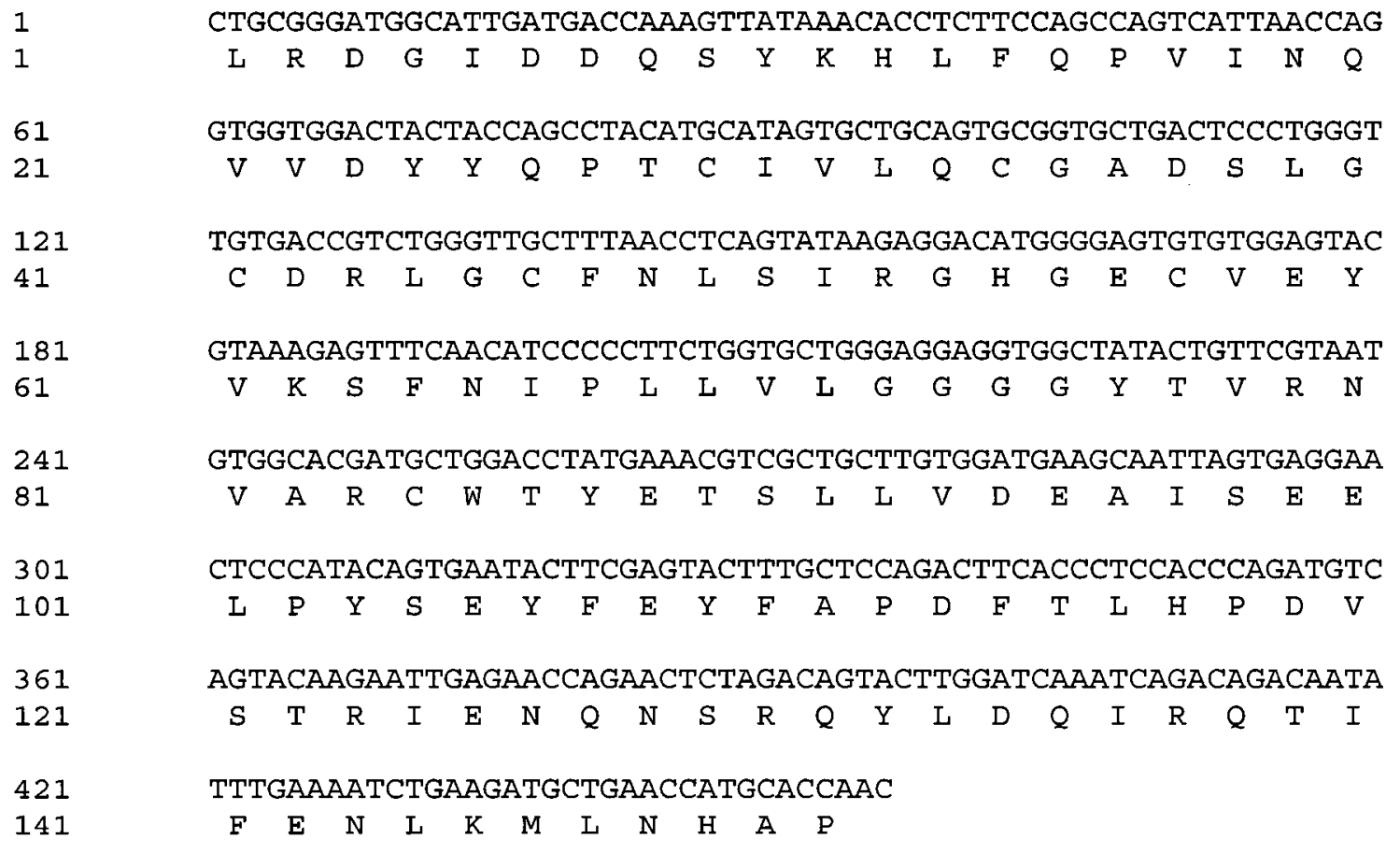

FIG.5.3. Nucleotide and deduced amino acid sequence for T. s. elegans partial Hdac3 sequence. Nucleotides and amino acids are numbered on the left. The nucleotide sequence was 453 nucleotides in length, while the amino acid sequence was 151 amino acids in length. 


\section{FIG.5.4}

1

1

61

21

121

41

181

61

241
CTACCAGGCATCAATGGAGGCAGCTGGCATTCCCCTGTCTTTTGGAGGACATCGGCCTCT

$\begin{array}{llllllllllllllllllll}Y & Q & A & S & M & E & A & A & G & I & P & L & S & F & G & G & H & R & P & L\end{array}$

GTCTCGTGCACAGTCCTCACCTGCATCTGCCACCTTCCCCATGTCCGTACAGGAGCCACC $\begin{array}{llllllllllllllllllll}S & R & A & Q & S & S & P & A & S & A & T & F & P & M & S & V & Q & E & P & P\end{array}$ AACTAAGCCGAGGTTCACAACAGGCCTTGTATACGACACCTTGATGCTGAAACACCAGTG $\begin{array}{llllllllllllllllllll}T & \mathrm{~K} & \mathrm{P} & \mathrm{R} & \mathrm{F} & \mathrm{T} & \mathrm{T} & \mathrm{G} & \mathrm{L} & \mathrm{V} & \mathrm{Y} & \mathrm{D} & \mathrm{T} & \mathrm{L} & \mathrm{M} & \mathrm{L} & \mathrm{K} & \mathrm{H} & \mathrm{Q} & \mathrm{C}\end{array}$ TACCTGTGGAAACACAAACAGCCACCCAGAGCATGCAGGGAGAATCCAGAGCATCTGGTC $\begin{array}{llllllllllllllllllll}T & C & G & N & T & N & S & H & P & E & H & A & G & R & I & Q & S & I & W & S\end{array}$ $\mathrm{CC}$

FIG. 5.4. Nucleotide and deduced amino acid sequence for $T$. s. elegans partial Hdac4 sequence. Nucleotides and amino acids are numbered on the left. The nucleotide sequence was 241 nucleotides in length, while the amino acid sequence was 80 amino acids in length. 


\section{FIG.5.5}

1

1

61

21

121

41

181

61

241

81

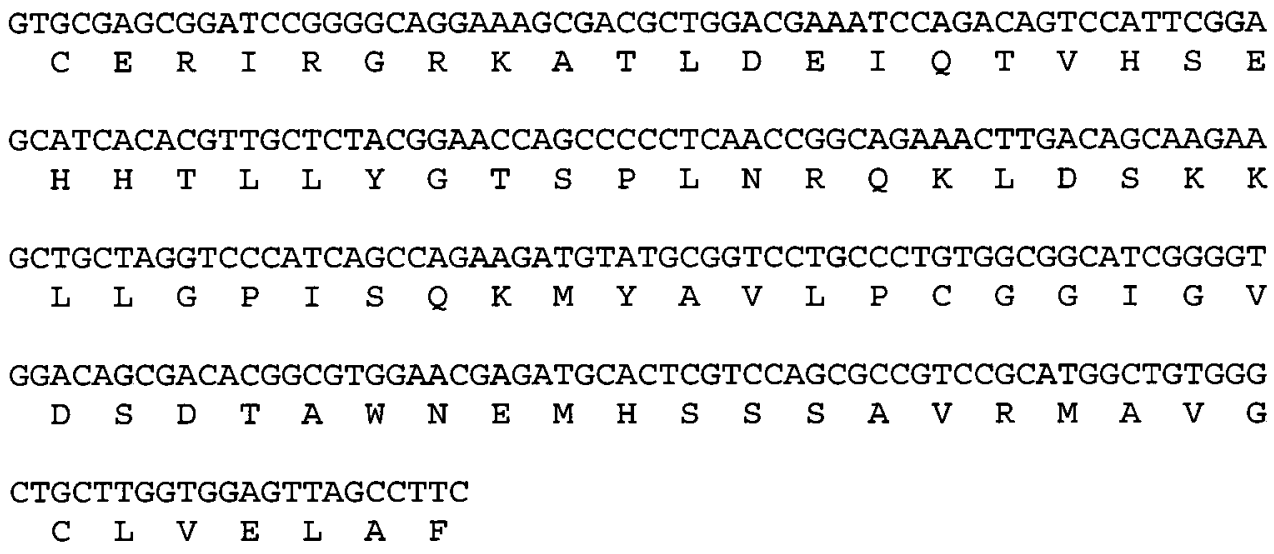

FIG. 5.5. Nucleotide and deduced amino acid sequence for T. s. elegans partial Hdac5 sequence. Nucleotides and amino acids are numbered on the left. The nucleotide sequence was 262 nucleotides in length, while the amino acid sequence was 87 amino acids in length. 
FIG.5.6
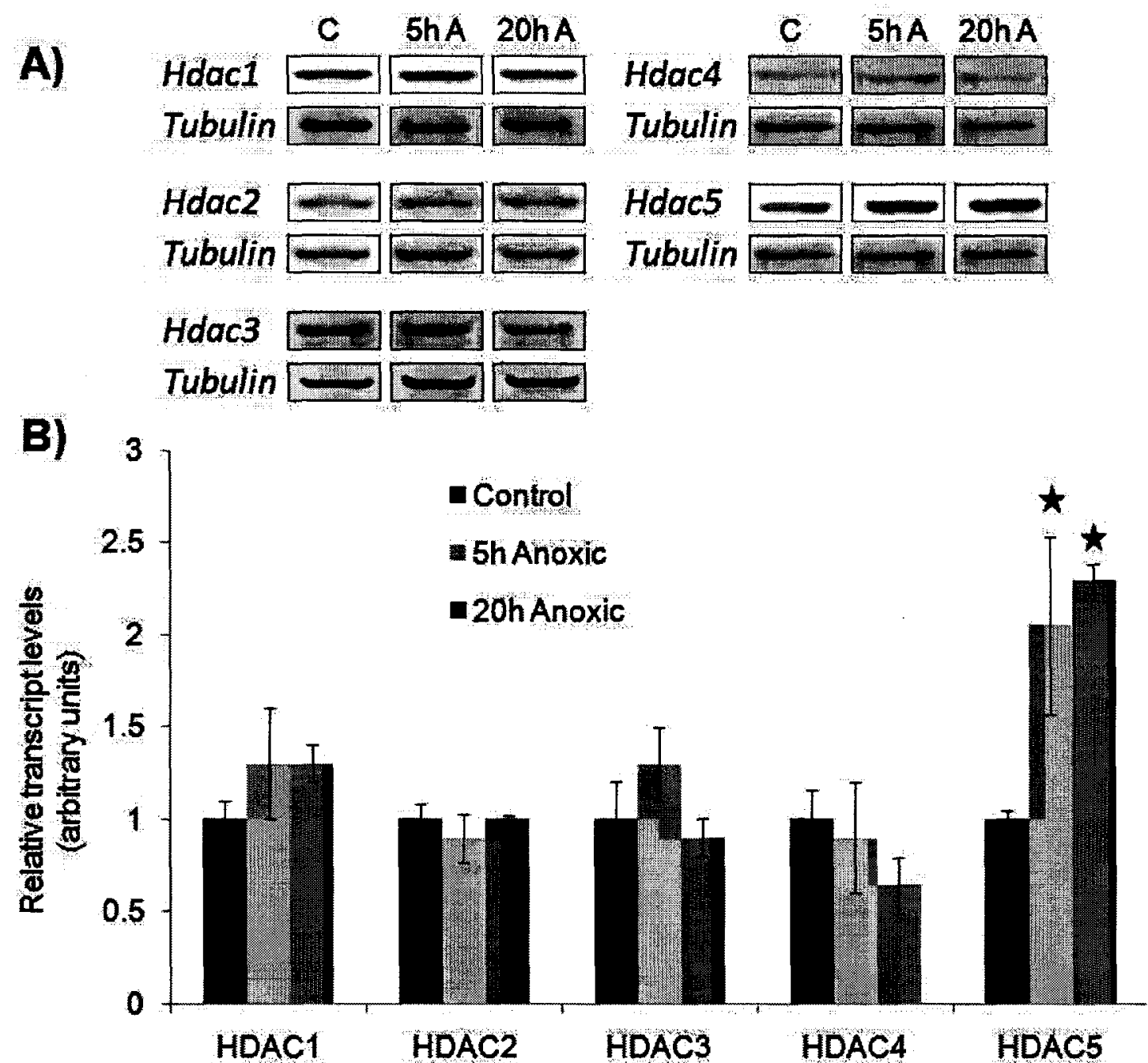

Fig. 5.6. Effect of 5 and $20 \mathrm{~h}$ of anoxic submergence on transcript levels of Hdac1-5 in T. s. elegans heart. (A) Representative RT-PCR bands of $H d a c$ amplicons with corresponding $\alpha$-tubulin bands under normoxia (C), $5 \mathrm{~h}$ of anoxia (5hA), and $20 \mathrm{~h}$ of anoxia (20hA). (B) Histogram showing normalized transcript levels under control and anoxic conditions; bands were normalized against the corresponding $\alpha$-tubulin bands amplified from the same sample. Data are means \pm S.E.M., $n=3-4$ independent trials on tissue from different animals. ${ }^{*}$ Significantly different from the corresponding control (P $<0.05)$. 
FIG.5.7
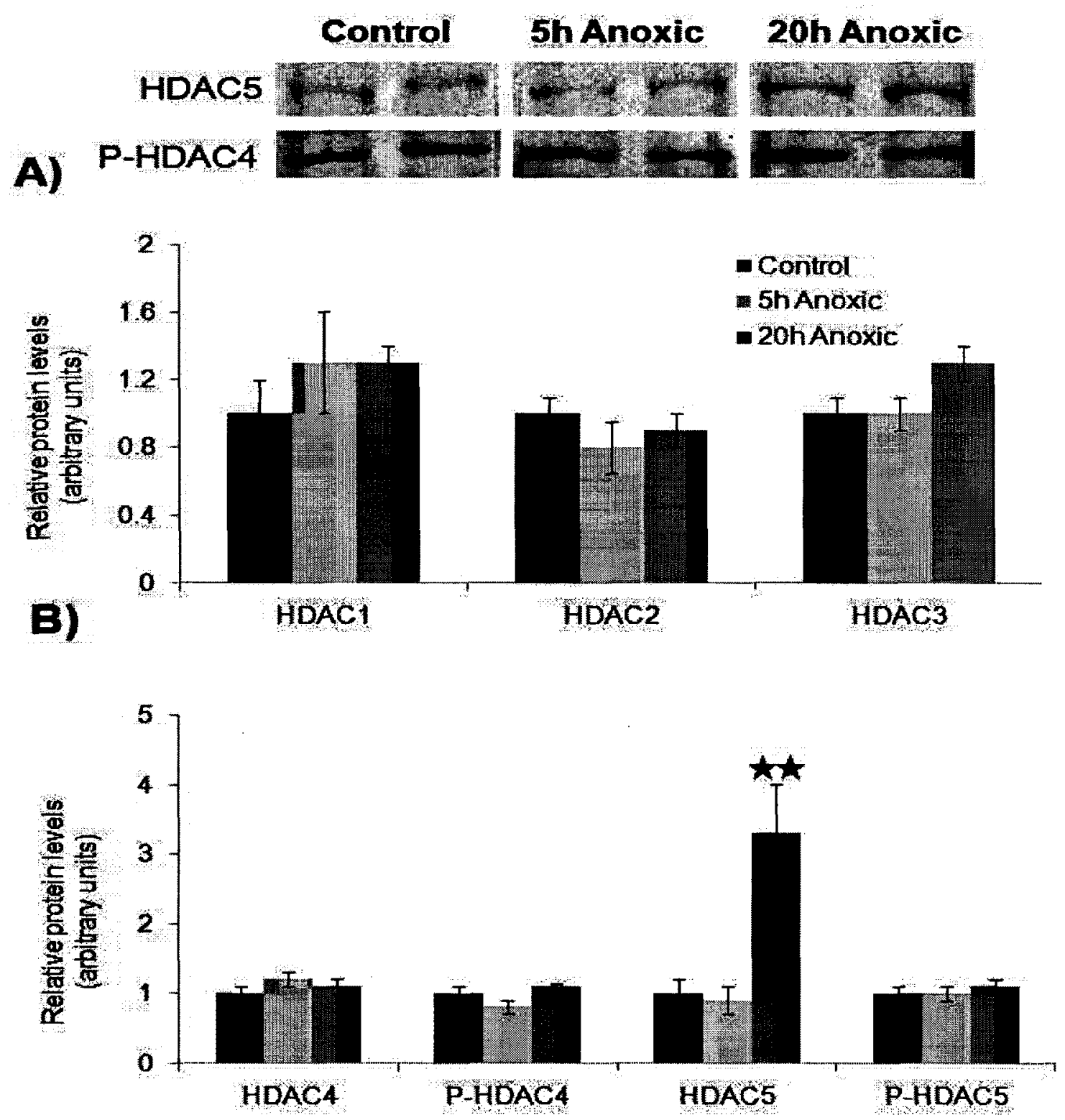

Fig. 5.7. Effect of 5 and $20 \mathrm{~h}$ of anoxic submergence on protein levels of HDAC1-5 in $T$. s. elegans heart. Representative immunoblots of heart HDAC5 and P-HDAC4 are shown ( $\mathrm{n}=2$ is shown). (A) Histogram showing normalized protein levels of class I HDACs, HDAC1-3. (B) Histogram showing normalized protein levels of class II HDACs, HDAC4-5 and their phosphorylated forms p-HDAC4 (Ser632) and p-HDAC5 (Ser498). Data are means \pm S.E.M., $n=3-4$ independent trials on tissue from different animals. $* *$ Significantly different from the corresponding control $(\mathrm{P}<0.01)$. 
FIG.5.8
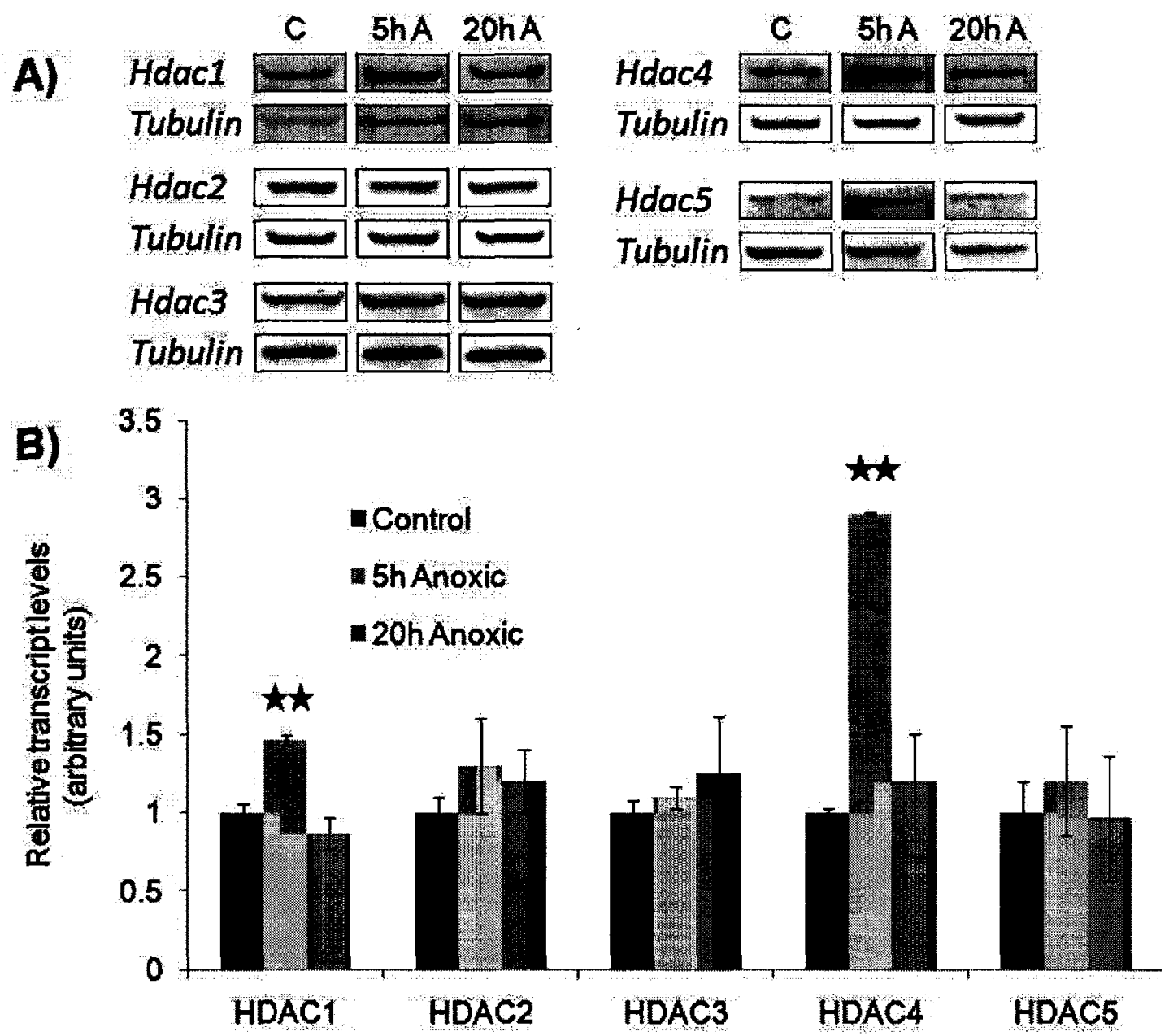

Fig. 5.8. Effect of 5 and $20 \mathrm{~h}$ of anoxic submergence on transcript levels of Hdacl-5 in liver of T. s. elegans. (A) Representative RT-PCR bands of Hdac amplicons with corresponding $\alpha$-tubulin bands under normoxia (C), $5 \mathrm{~h}$ of anoxia (5hA), and $20 \mathrm{~h}$ of anoxia (20hA). (B) Histogram showing normalized transcript levels under control and anoxic conditions; bands were normalized against the corresponding $\alpha$-tubulin bands amplified from the same sample. Data are means \pm S.E.M., $n=3-4$ independent trials on tissue from different animals. ** Significantly different from the corresponding control (P $<0.01)$. 
FIG.5.9

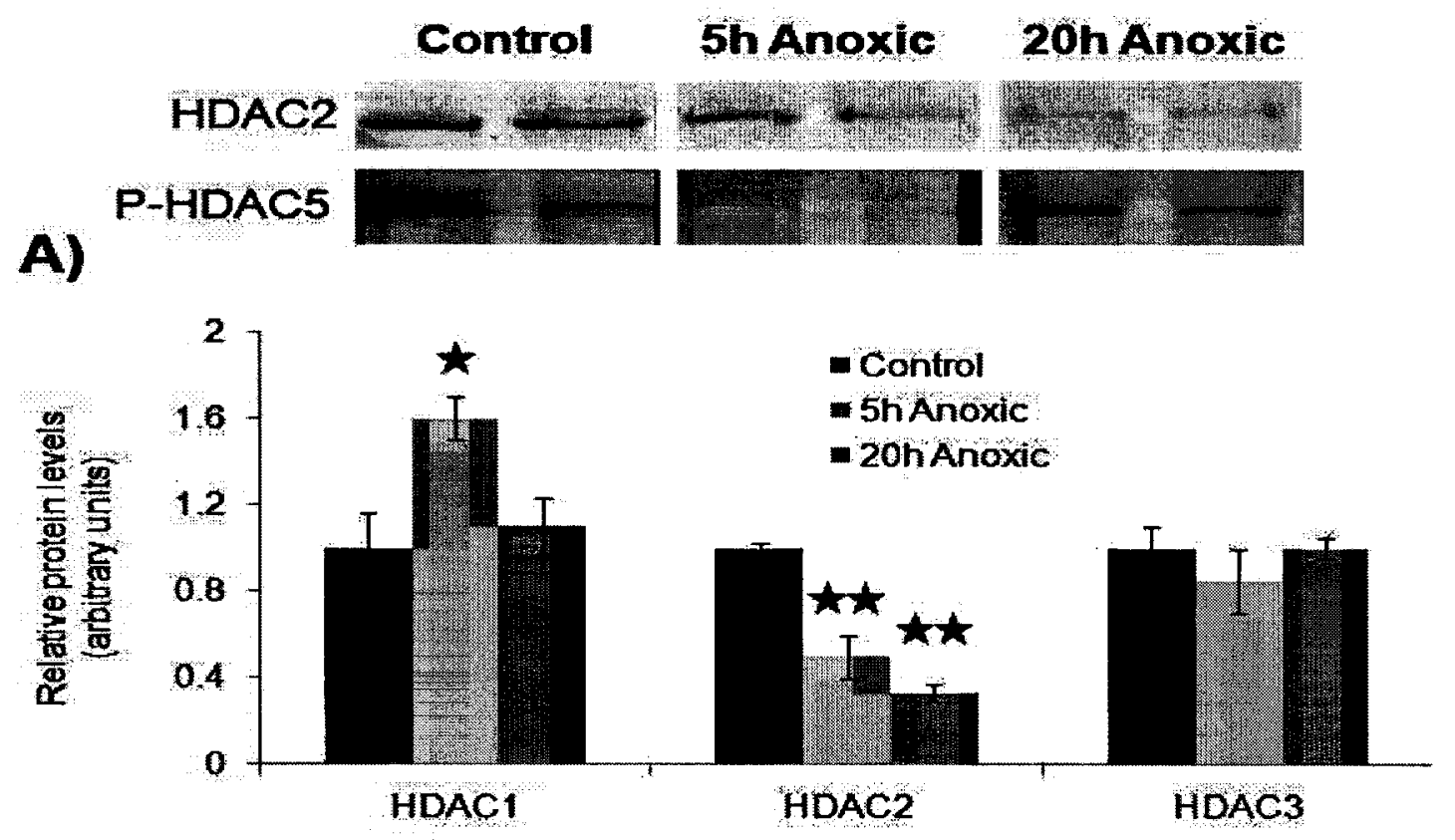

B)

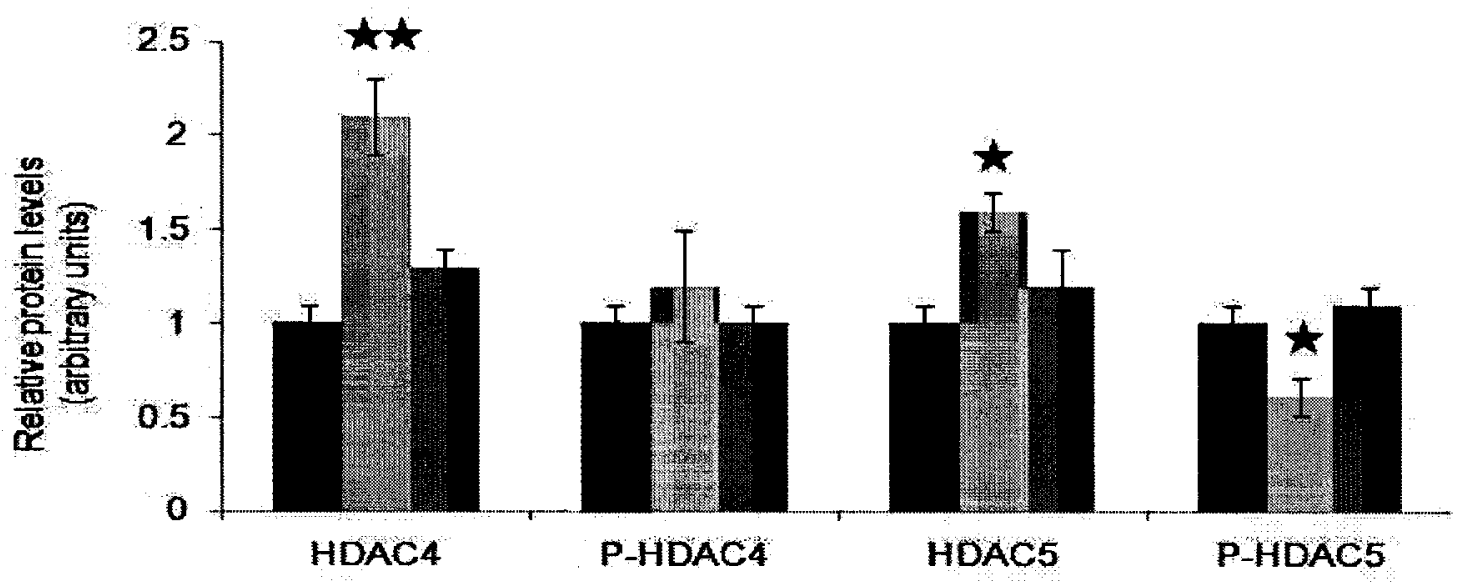

Fig. 5.9. Effect of 5 and $20 \mathrm{~h}$ of anoxic submergence on protein levels of HDAC1-5 in liver of $T$. s. elegans. Representative immunoblots of HDAC2 and P-HDAC5 are shown ( $\mathrm{n}=2$ is shown).

(A) Histogram showing normalized protein levels of class I HDACs, HDAC1-3.

(B) Histogram showing normalized protein levels of class II HDACs, HDAC4-5 and their phosphorylated forms p-HDAC4 (Ser632) and p-HDAC5 (Ser498). Data are means \pm S.E.M., $n=3-4$ independent trials on tissue from different animals. * Significantly different from the corresponding control $(\mathrm{P}<0.05)$; ${ }^{* *}$ significantly different from the corresponding control $(\mathrm{P}<0.01)$. 
FIG.5.10

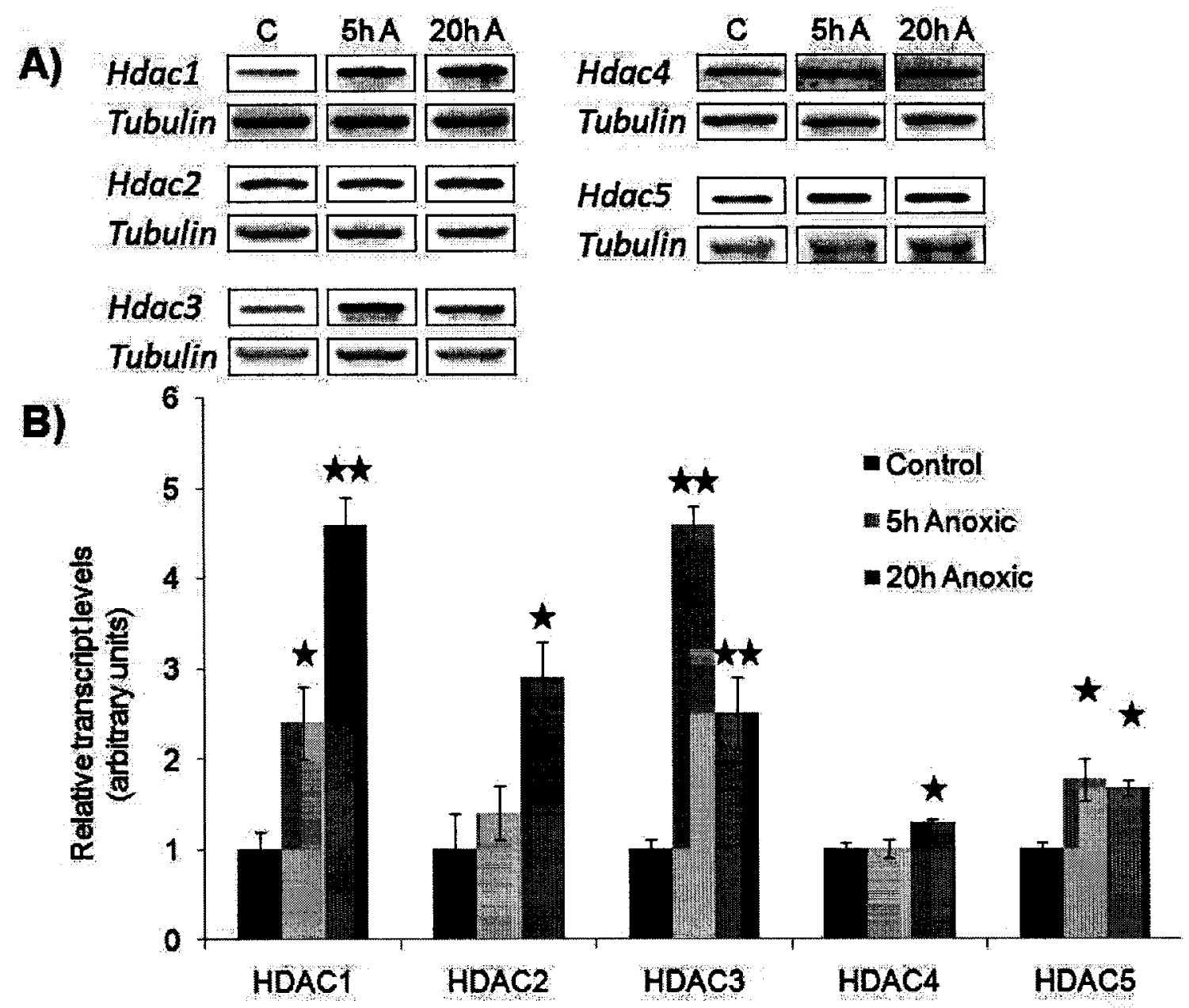

Fig. 5.10. Effect of 5 and $20 \mathrm{~h}$ of anoxic submergence on transcript levels of Hdac1-5 in white skeletal muscle of $T$. s. elegans. (A) Representative RT-PCR bands of Hdac amplicons with corresponding $\alpha$-tubulin bands under normoxia (C), $5 \mathrm{~h}$ of anoxia (5hA), and $20 \mathrm{~h}$ of anoxia (20hA). (B) Histogram showing normalized transcript levels under control and anoxic conditions; bands were normalized against the corresponding $\alpha$ tubulin bands amplified from the same sample. Data are means \pm S.E.M., $n=3-4$ independent trials on tissue from different animals. * Significantly different from the corresponding control $(\mathrm{P}<0.05) ; * *$ significantly different from the corresponding control $(\mathrm{P}<0.01)$. 
FIG.5.11

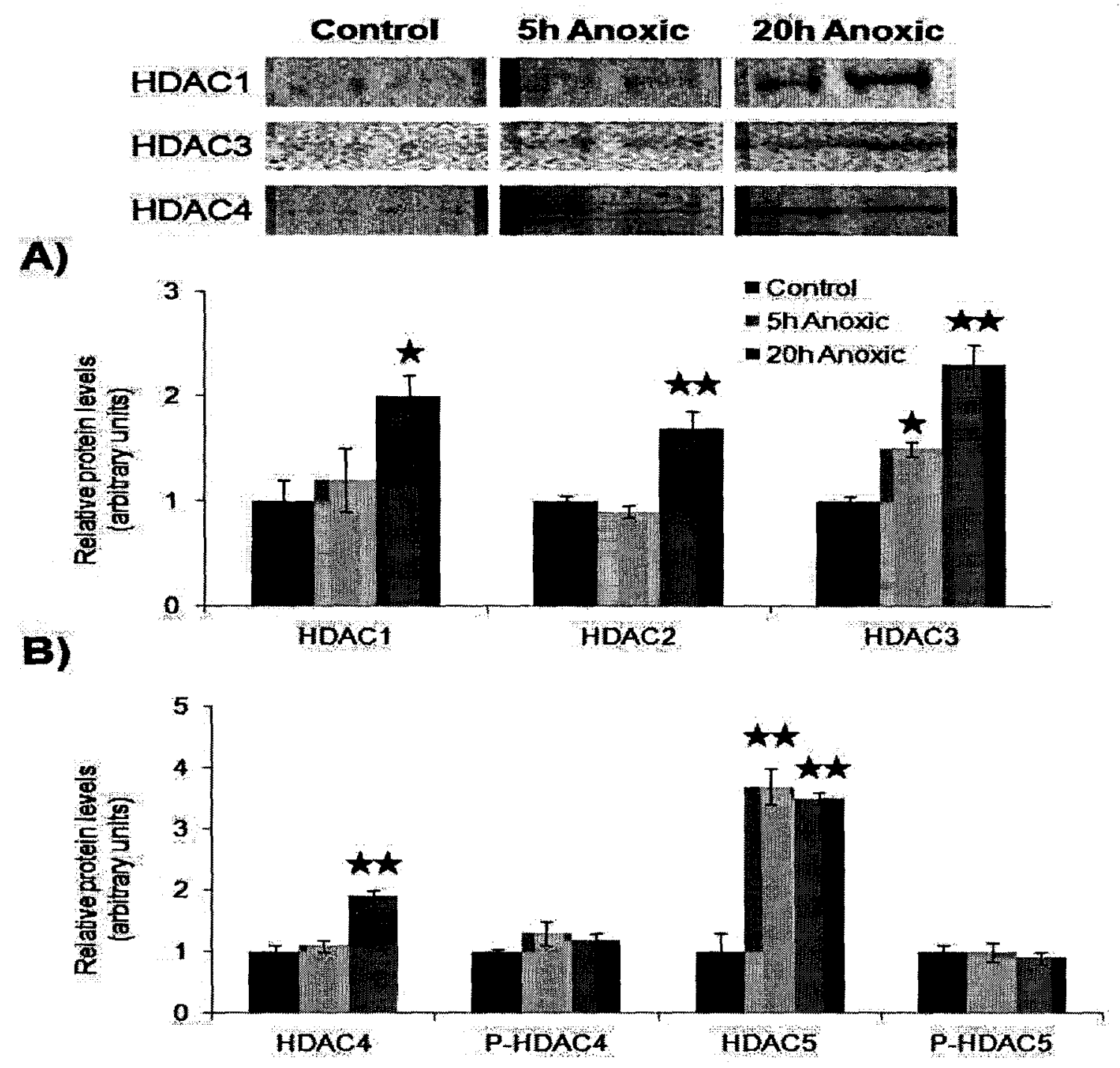

Fig. 5.11. Effect of 5 and $20 \mathrm{~h}$ of anoxic submergence on protein levels of HDAC1-5 in white skeletal muscle of $T$. s. elegans. Representative immunoblots of HDAC1, 3 and 4 are shown ( $\mathrm{n}=2$ is shown). (A) Histogram showing normalized protein levels of class $\mathrm{I}$ HDACs, HDAC1-3. (B) Histogram showing normalized protein levels of class II HDACs, HDAC4-5 and their phosphorylated forms p-HDAC4 (Ser632) and p-HDAC5 (Ser498). Data are means \pm S.E.M., $n=3-4$ independent trials on tissue from different animals. * Significantly different from the corresponding control $(\mathrm{P}<0.05)$; ${ }^{* *}$ significantly different from the corresponding control $(\mathrm{P}<0.01)$. 
FIG.5.12

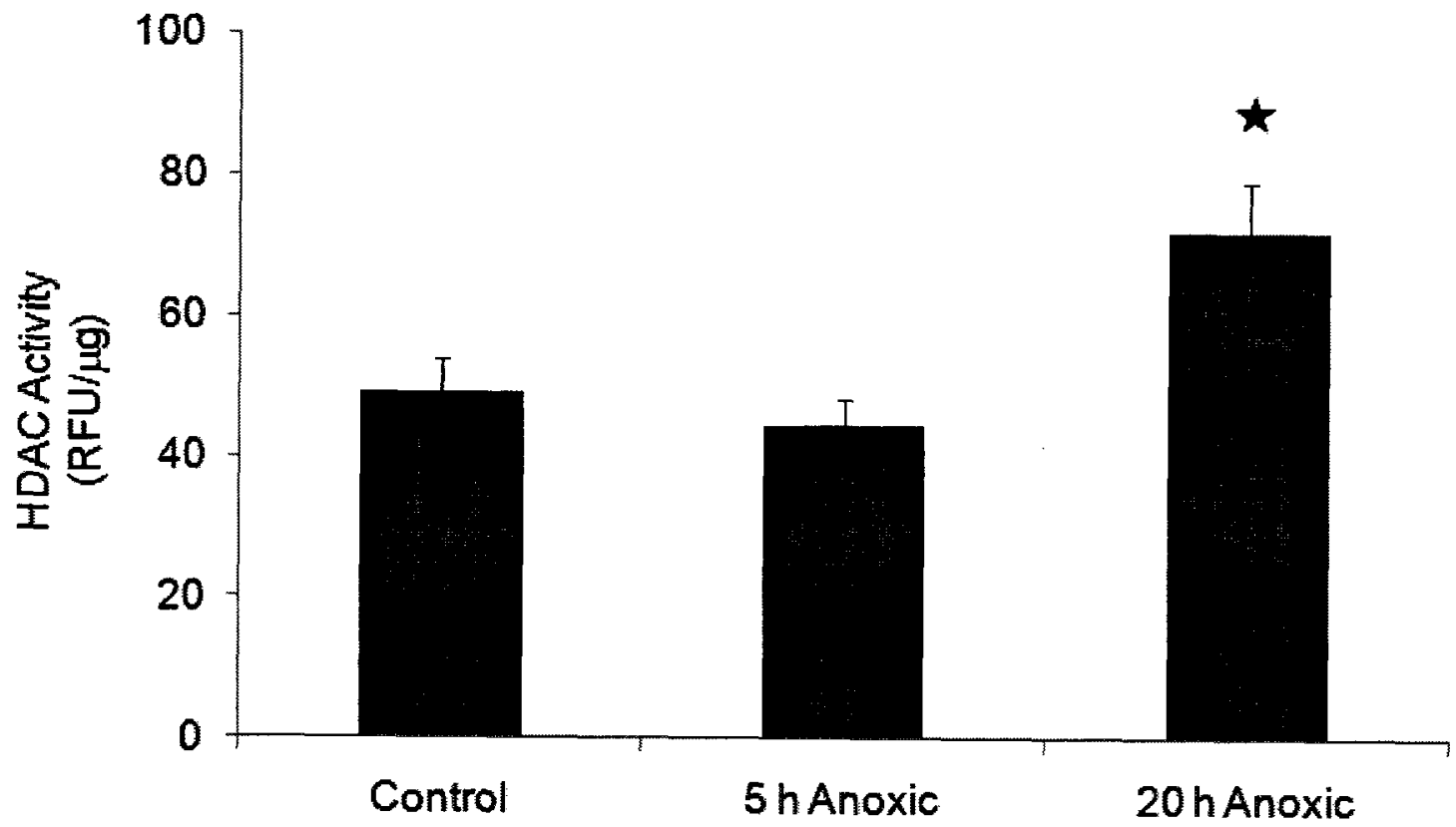

Fig. 5.12. Effect of 5 and $20 \mathrm{~h}$ of anoxic submergence on HDAC activity (expressed in relative fluorescence units per microgram protein) in white skeletal muscle of $T . s$. elegans. Data are means \pm S.E.M., $n=4$ independent trials on tissue from different animals. * Significantly different from the corresponding control $(P<0.05)$. 
FIG.5.13
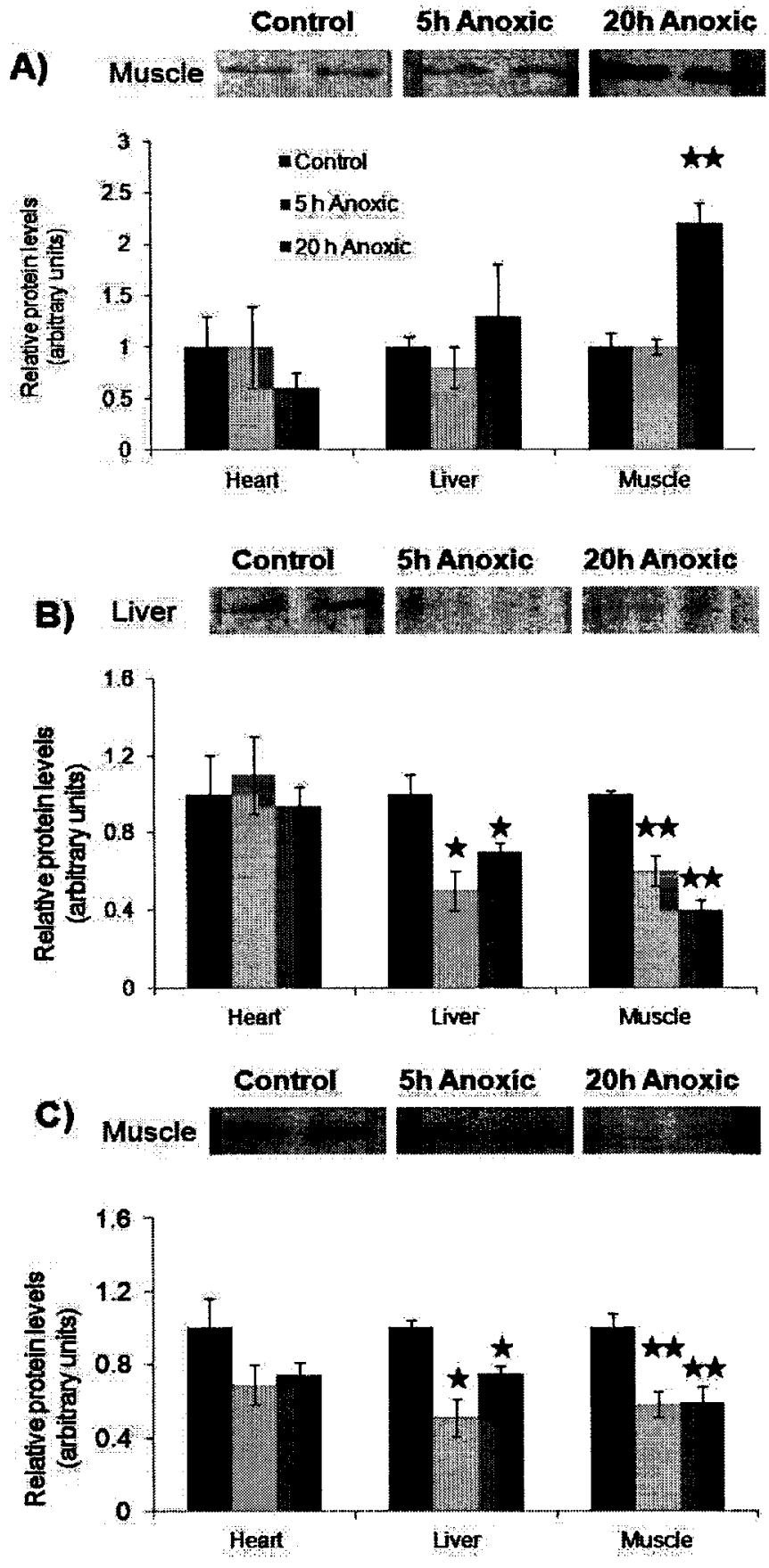

Fig. 5.13. Effect of 5 and $20 \mathrm{~h}$ of anoxic submergence on total and acetylated histone $\mathrm{H} 3$ expression in the heart, liver, and white skeletal muscle of $T$. s. elegans. (A) Histogram showing normalized total histone $\mathrm{H} 3$ levels. A representative immunoblot of histone $\mathrm{H} 3(\mathrm{n}=2)$ in muscle is shown. (B) Histogram showing normalized levels of acetylated histone $\mathrm{H} 3$ (Lys 9). A representative immunoblot of acetyl histone H3, Lys9 $(\mathrm{n}=2)$ in liver is shown. (C) Histogram showing normalized levels of acetylated histone H3 (Lys 23). A representative immunoblot of acetyl histone H3, Lys $23(n=2)$ in muscle is shown. Data are means \pm S.E.M., $n=3-4$ independent trials on tissue from different animals. * Significantly different from the corresponding control $(\mathrm{P}<0.05)$; ${ }^{* *}$ significantly different from the corresponding control $(\mathrm{P}<0.01)$. 


\section{CHAPTER 6}

\section{Changing metabolism: activation of ChREBP in response to anoxia}

(Publication in preparation) 


\subsection{INTRODUCTION}

An important aspect of anoxia survival is the readjustment of metabolism to optimize efficiency under the anoxic state. Under aerobic conditions, ATP can be generated by oxidizing multiple fuel sources, including carbohydrates, lipids and amino acids. By contrast, under anoxia, oxidative phosphorylation becomes impossible due to its reliance on oxygen as a final electron acceptor and anaerobic glycolysis becomes the sole mechanism for producing ATP in the cell. As a result, carbohydrates (in the form of glucose and glycogen) become the sole fuel source of the cell. Therefore, it is expected that as the turtle enters the anoxic state, it will re-organize its energy producing pathways to facilitate optimal carbohydrate catabolism. In addition, studies have shown that metabolic priorities are also reorganized under anoxia, providing both global suppression of metabolic functions and a reprioritization of ATP use by different specific cell functions (Hochachka et al., 1996). Reversible protein phosphorylation of key enzymes and functional proteins has been shown to be a key mechanism for this metabolic reorganization (Storey, 1996). However, the transcriptional responses that underlie metabolic reorganization in anoxia have not been explored. The present chapter presents the first study to examine the response to anoxia of an important transcription factor involved in glucose metabolism.

ChREBP (Carbohydrate Response Element Binding Protein) is a glucoseresponsive protein that is predominately expressed in liver, kidney, and adipose tissue (Towle, 2005). In recent years, ChREBP has emerged as an important transcription factor implicated in the regulation of glycolytic and lipogenic genes by glucose (Yamashita et al., 2001; Ishii et al., 2004; Dentin et al., 2004, 2005). It does so by binding to glucose- 
responsive DNA elements, termed carbohydrate response elements (ChRE) in the promoter regions of these genes (Bergot et al., 1992; Shih et al., 1994; Rufo et al., 2001). ChREBP contains several phosphorylation sites for cAMP-dependent protein kinase (PKA) and AMP-activated protein kinase (AMPK) (Kawagushi et al., 2001) and its phosphorylation is thought to be regulated by these kinases. According to the present model, PKA targets the serine 196 residue on ChREBP, located near the nuclear localization sequence. It is thought that under basal conditions, ChREBP is phosphorylated on Ser-196, resulting in cytoplasmic localization (FIG.6.1). By contrast, high glucose conditions prompt the activation of protein phosphatase 2A (PP2A) by xylulose-5-phosphate (X5P), an intermediate of the pentose phosphate pathway, which then dephosphorylates ChREBP on Ser-196, allowing it to localize to the nucleus (Kabashima et al., 2003). Once in the nucleus, a second dephosphorylation on Thr-666 enhances the DNA-binding activity of ChREBP, allowing it to bind to the ChRE in the promoters of glycolytic and lipogenic genes (FIG.6.1; Kawagushi et al., 2001).

One major glycolytic target gene of ChREBP is the liver isozyme of pyruvate kinase (LPK). Pyruvate kinase is a regulatory enzyme that contributes to the control of flux through glycolysis together with phosphofructokinase and hexokinase (or glycogen phosphorylase depending on the carbon source) (Allert et al., 1991). Inhibitory control over PK in liver is also critical to promoting gluconeogenesis from products including lactate and alanine. PK catalyzes the formation of pyruvate and ATP from phosphoenolpyruvate (PEP) and ADP. The enzyme has four isozymes, L, R, M1 and M2 that are expressed in different tissues (Imamura et al., 1986). LPK is expressed predominately in the liver, but is also found in kidney, small intestine and pancreatic beta 
cells (Imamura et al., 1986; Noguchi et al., 1991). PK activity can be regulated in response to various hormones, nutrients and stresses by changes in its expression levels (protein synthesis and degradation), post-translational modifications (mainly reversible phosphorylation), and allosteric effectors (e.g. fructose-1,6-bisphosphate activation, alanine inhibition) (Yamada and Nugochi, 1999). For example, under low glucose conditions, high glucagon levels lead to an elevation in cAMP levels in the cell; cAMP in turn stimulates phosphorylation of LPK by PKA to inhibit its activity (thereby, helping to direct glycogenolysis towards glucose export) (El-Maghrabi et al., 1980; Ishibashi and Cottam, 1978). In contrast, under high glucose conditions, insulin activates LPK by stimulating its dephosphorylation so that glucose can be made available for lipogenic and other biosynthetic needs (Assimacopoulos-Jeannet and Jeanrenaud, 1990).

Because of the pivotal role of glycolysis in energy production during anoxia, and because of the importance of ChREBP in the regulation of glycolytic enzyme transcription, I hypothesized that this transcription factor will be activated in response to anoxia in turtle tissues. The present chapter analyzes ChREBP responses by examing its transcript and protein expression, nuclear localization, and DNA-binding activity, as well as the transcript levels of its target gene LPK, in T. s. elegans kidney and liver in response to anoxia. 


\subsection{MATERIALS AND METHODS}

\subsubsection{Animals}

Turtle experiments and tissue handling were as described in Chapter 2. Kidney and liver were the only tissues used for this chapter.

\subsubsection{RNA extraction and $c D N A$ synthesis}

Total RNA was isolated from turtle tissues as described in Chapter 2. cDNA was reverse transcribed and serially diluted.

\subsubsection{Primers}

The ChREBP forward and reverse primers were designed from a consensus sequence of mammalian and chicken ChREBP using the Primer Designer program, version 3.0 (Scientific and Educational Software). The nucleotide sequence of the forward ChREBP primer was 5'-CACAGCGGWCACTTCATGGT-3' (W= A or T) whereas the reverse primer sequence was 5'- GGTCYGGCTGGATCATGTCA -3' (Y= $\mathrm{C}$ or T). The $L P K$ primers were designed based on partial mRNA sequence for $L P K$ available from NCBI (accession\#: $\underline{\mathbf{A J 2 4 3 1 3 7}}$ ). The nucleotide sequence of the forward $L P K$ primer was 5'- GGCGAGAACATGGAGGTGGA -3 whereas the reverse primer sequence was 5'-CCACGAGCCACCATTATTCC -3'. Primers were purchased from Sigma Genosys. Primers for the control gene $\alpha$-tubulin were as described in Chapter 3. 


\subsection{4. $R T-P C R$}

PCR reactions were composed as described in Chapter 2. The PCR program was as follows: 7 min at $94^{\circ} \mathrm{C}$ followed by 36 cycles of $1 \mathrm{~min}$ at $94^{\circ} \mathrm{C}, 1 \mathrm{~min}$ at $54^{\circ} \mathrm{C}$ and $1.5 \mathrm{~min}$ at $72^{\circ} \mathrm{C}$. The final step was $72^{\circ} \mathrm{C}$ for $10 \mathrm{~min}$. PCR products were separated on a $1 \%$ agarose gel stained with ethidium bromide, visualized using the ChemiGenius imaging system (Syngene, Frederick, MD, USA) under UV light and quantified using the GeneTools program. Bands from the most dilute cDNA sample that gave visible product were used for quantification to make sure that the products had not reached amplification saturation.

PCR products were sequenced by StemCore Laboratories (Ottawa, ON). The sequences were verified as encoding ChREBP and $L P K$ using the program BLASTN (http://www.ncbi.nlm.nih.gov/blast) at the NIH.

\subsubsection{Protein extracts and western blotting}

Protein extracts were prepared from tissue samples of aerobic, $5 \mathrm{~h}$ and $20 \mathrm{~h}$ anoxic turtles, as described in Chapter 2. Aliquots containing $30 \mu \mathrm{g}$ protein were loaded into lanes of $10 \%$ SDS polyacrylamide gels, electrophoresed, and electroblotted onto polyvinylidene difluoride (PVDF) membranes, also as described previously. After the transfer of proteins, membranes were blocked for $10 \mathrm{~min}$ in Tris buffered saline containing Tween-20 (TBST: $20 \mathrm{mM}$ Tris base, $140 \mathrm{mM} \mathrm{NaCl}, 0.1 \% \mathrm{v} / \mathrm{v}$ Tween-20) with $2.5 \%$ non-fat dried milk added. The blots were then probed overnight with primary antibody for ChREBP (Santa Cruz Biotechnology; diluted 1:1000 in TBST). After incubation, blots were washed several times with TBST and then incubated at room 
temperature for 45 min with HRP-linked donkey anti-goat IgG (Santa Cruz

Biotechnology; diluted 1:2000 in TBST). Immunoblots were developed using enhanced chemiluminescence $(\mathrm{ECL})$ reagents.

\subsubsection{Preparation of nuclear extracts}

Cytoplasmic and nuclear extracts from kidney and liver of aerobic and anoxic turtles were prepared as described in Chapter 2.

\subsubsection{DNA-Binding Assay}

Nuclear extracts from kidney and liver for the DNA-binding assay were prepared as described in chapter 2. Aliquots containing equal amounts of protein from each sample were then used to assess the amount of binding by ChREBP to its response element using a DNA-binding assay kit from Cayman Chemical (Ann Arbor, MI). Binding assays were performed according to the manufacturer's protocol. Briefly, ChREBP binding (16 $\mu \mathrm{g}$ of protein) was carried out for $1.5 \mathrm{~h}$ at room temperature with mild agitation on a rocking platform. The plates were then washed with wash buffer to remove unbound transcription factors. ChREBP-specific primary antibody diluted 1:100 v/v in antibody buffer was then added to each well and incubated for $1 \mathrm{~h}$ at room temperature without agitation. The wells were then washed three times with washing buffer and the secondary antibody (HRP-linked anti-rabbit antibody diluted 1:100 v/v in antibody buffer) was then added to each well and incubated for $1 \mathrm{~h}$ at room temperature without agitation. The wells were then washed five times with washing buffer and the manufacturer's developing solution was then added. After developing for $45 \mathrm{~min}$ in the dark, the stop solution was added and 
colour development was quantified by absorbance readings at $450 \mathrm{~nm}$. Absorbance values (minus blanks) are proportional to the amount of DNA-binding over a wide range.

\subsubsection{Data Analysis}

All data were analyzed as described in Chapter 2. Significant differences among groups containing three conditions were assessed using analysis of variance followed by the Dunnett's post-hoc test. Significant differences between groups containing two experimental conditions were determined using the Student's t-test. Statistical difference was accepted if $\mathrm{P}<0.05$.

\subsection{RESULTS}

\subsection{1. cDNA cloning of ChREBP}

Using RT-PCR and the primers derived from the consensus sequence of ChREBP from other vertebrates, a $648 \mathrm{bp}$ PCR product was retrieved from total RNA prepared from $T$. s. elegans liver. The product was confirmed as encoding a portion of ChREBP using BLAST. FIG.6.2 shows the partial nucleotide sequence of ChREBP with the corresponding amino acid sequence. FIG.6.3 shows the translated amino acid sequence of turtle ChREBP aligned with sequences from chicken, human, and mouse, whereas FIG.6.4 shows a homology tree (FIG.6.4A) and a homology matrix (FIG6.4B) comparing the percent identities for the four protein sequences. Turtle ChREBP amino acid sequence was the closest to that of the chicken, showing an $82 \%$ identity, with 77 $79 \%$ identity with the mammalian sequences. 
The turtle sequence showed seven unique amino acid substitution compared to the other vertebrates; these are bold/shaded in FIG.6.3. These were substitutions of glycine, histidine, threonine, leucine, glutamic acid, lysine and asparagine, respectively, as compared with lysine, arginine, serine, proline, glutamic acid, arginine and glutamic acid the other vertebrate sequences. Of additional note is the preservation in the turtle sequence of the PKA phosphorylation site that is seen at Ser-196 in mammals and Ser197 of the chicken sequence.

\subsubsection{Expression of ChREBP in response to anoxia}

Transcript levels of chrebp in kidney and liver from normoxic, $5 \mathrm{~h}$ anoxic and 20 $\mathrm{h}$ anoxic T. s. elegans were assessed using RT-PCR (FIG.6.5A). Alpha-tubulin mRNA, a constitutively expressed gene, was also amplified from the same samples and ChREBP transcript levels were normalized against the tubulin transcript level in each sample. Transcript levels of chrebp in increased slightly in the kidney by $1.2 \pm 0.03$ fold as compared with controls in response to $5 \mathrm{~h}$ anoxia and remained at $1.2 \pm 0.03$ fold higher (both $\mathrm{P}<0.05$ as compared with controls). In the liver, chrebp transcripts increased to 1.8 \pm 0.2 fold of control in response to $5 \mathrm{~h}$ of anoxia but returned to normoxic levels by $20 \mathrm{~h}$ of anoxia.

ChREBP protein levels were assessed via western blotting in kidney and liver extracts from normoxic, $5 \mathrm{~h}$ anoxic, and $20 \mathrm{~h}$ anoxic T. s. elegans (FIG.6.5B). The polycolonal antibody crossreacted with a band of $95 \mathrm{kDa}$ that corresponded with the known molecular weight for ChREBP in other vertebrates. In both tissues, ChREBP protein levels increased significantly after $5 \mathrm{~h}$ anoxia but had returned to control values 
by $20 \mathrm{~h}$. After $5 \mathrm{~h}$ anoxia exposure ChREBP protein values were $1.9 \pm 0.2$ fold higher than the normoxic level in kidney $(\mathrm{P}<0.05)$ and $1.8 \pm 0.2$ fold higher than controls in liver $(\mathrm{P}<0.05)$.

\subsubsection{Subcellular distribution of ChREBP}

The subcellular distribution of ChREBP in kidney and liver of normoxic and $5 \mathrm{~h}$ anoxic turtles was assessed using western blotting. FIG.6.6A shows the relative protein levels of ChREBP in the cytoplasm and nucleus of kidney during normoxia and anoxia, whereas FIG.6.6B shows the comparable data for liver. Cytoplasmic levels of ChREBP in kidney remained unchanged between normoxic and anoxic conditions, but the nuclear ChREBP content increased significantly during anoxia to $2.8 \pm 0.1$ fold higher than controls $(\mathrm{P}<0.05)$. This increase indicates translocation of ChREBP to the nucleus. Liver ChREBP was elevated in both compartments in response to $5 \mathrm{~h}$ anoxia; cytoplasmic and nuclear ChREBP levels rose by $1.8 \pm 0.07$ and $2.2 \pm 0.3$ fold, respectively $(\mathrm{P}<0.05)$.

\subsubsection{DNA binding activity of ChREBP}

Glucose-mediated activation of ChREBP results in both nuclear translocation, as well as enhanced DNA-binding activity, inducing the transcription of downstream genes. Relative changes in ChREBP DNA binding activity between normoxic and $5 \mathrm{~h}$ anoxic states were assessed in turtle kidney and liver (FIG.6.7). Kidney showed no significant change in the DNA binding activity of ChREBP between the two states. By contrast, the 
liver showed a significant increase in the DNA binding activity of ChREBP during anoxia, rising by $4.2 \pm 0.6$ fold as compared with the control $(\mathrm{P}<0.05)$.

\subsection{5. cDNA cloning and $m R N A$ levels of $L P K$}

The primers designed for $L P K$ were based on a $T$. s. elegans LPK sequence obtained from GeneBank (accession number: AJ243137). A 415 bp PCR product was retrieved from total RNA prepared from turtle liver and was confirmed as $L P K$ by BLAST.

Transcript levels of $L P K$ in livers from normoxic and anoxic turtles were assessed using RT-PCR (FIG.6.8). LPK transcripts in liver rose to $2.1 \pm 0.1$ fold higher than control values $(\mathrm{P}<0.05)$ after $5 \mathrm{~h}$ anoxia but returned to normoxic values by $20 \mathrm{~h}$.

\subsection{DISCUSSION}

Several important processes unfold when animals are subjected to oxygen limitation. Inability to proceed with oxidative phosphorylation renders anaerobic glycolysis the chief energy producer in the cell, creating a need for a higher glycolytic flux to compensate for energy demands. A higher glycolytic flux can be obtained by changing the properties of the regulatory enzymes of glycolysis, as well as upregulating the levels of these enzymes (as mediated by HIF). In anoxia-intolerant animals (such as mammals) elevated levels of glycolysis cannot be sustained for long because of the inefficiency of glycolysis, insufficiency of substrate stores (glucose, glycogen), and the accumulation of toxic end products. Turtles have solved these problems through several 
mechanisms, including large glycogen stores in the liver that fuel both liver glycolysis and provide glucose for export to other organs, buffering and removal of glycolytic end products, and very strong metabolic rate depression that can lower energy demands in anoxia to just $10-20 \%$ of the aerobic rate (Storey, 1996). However, little is known to date about metabolic adjustments at the gene level. The present chapter investigates ChREBP, a pivotal transcription factor that regulates carbohydrate metabolism, and its potential role in anoxia tolerance.

ChREBP has important roles in regulating enzymes of glycolysis and lipogenesis. To date its function has been primarily investigated in the liver, due to this organ's key role in carbohydrate metabolism. However, this transcription factor is expressed in other tissues as well, and is of potential importance in them. The present model of ChREBP regulation suggests that under basal conditions it is found in the cytoplasm in a phosphorylated state (Kabashima et al., 2003). High glucose concentrations result in dephosphorylation of ChREBP on serine 196, allowing it to change conformation and translocate into the nucleus. Once in the nucleus, high glucose concentrations drive a further dephosphorylation of ChREBP on the threonine 666 residue, allowing it to acquire DNA-binding activity and mediate transcription (FIG.6.1) (Kawagushi et al., 2001).

A partial sequence of $T$. s. elegans ChREBP was obtained using RT-PCR (FIG. 6.2) and aligned with sequences from other vertebrates for comparison. Unfortunately, apart from the mammalian sequences only chicken ChREBP was available for comparison, so turtle ChREBP could not be compared to a broad spectrum of vertebrates. Among the species used, the partial turtle amino acid sequence was closest to the 
chicken, sharing $83 \%$ homology with $77-79 \%$ identity with the mammalian sequence (FIG.6.4). Significantly, the known PKA phosphorylation site (Ser-196 in mammals, Ser-197 in chicken), and its surrounding amino acids, was conserved which argues that turtle liver ChREBP would likely be regulated by PKA.

FIG.6.3 shows the amino acid alignment of the partial ChREBP sequence compared to the other vertebrates. Interestingly, the turtle sequence displayed many unique amino acid substitutions (shaded and bolded in the figure). Among these, several could have potentially significant effect on the structural properties of ChREBP. For example, one such substitution is a leucine in the turtles compared to proline in the other vertebrates. While both amino acids are nonpolar and neutral, leucine is hydrophobic, while proline is hydrophilic. Moreover, these amino acids are quite different structurally. Another potentially significant substitution is a glutamic acid in the turtle compared to proline in the other vertebrates. Glutamic acid is polar and negative, while proline is nonpolar and neutral. Difference in the properties of some amino acids between the turtle sequence and the other vertebrates could potentially reflect an evolutionary adjustment to enhance protein stabilization under the anoxic conditions. However, it is also possible that these substitutions are not turtle-specific but are merely ectotherm-specific, or even random, and might not be associated with anoxia tolerance. Evidently, the significance of these substitutions will require that more sequence data for other vertebrates become available.

To assess ChREBP response to anoxia, I initially examined its transcript and protein expression in turtle kidney and liver. Interestingly, both mRNA and protein levels of ChREBP increase in response to anoxia in both tissues (FIG.5), suggesting that 
regulation at the expression level is a potentially important mechanism of ChREBP activation. This suggests that some hypoxia/anoxia-responsive upstream factor(s) controls the expression of ChREBP and its upregulation in response to anoxia. The liver displayed an increase in ChREBP expression in response to $5 \mathrm{~h}$ of anoxic submergence, with transcript and proteins levels each increasing by 1.8 fold. Furthermore, there was strong evidence of enhanced transcriptional activity by ChREBP during short term anoxia: a 2.2-fold increase in nuclear ChREBP levels (FIG.6.6), a 4.2 fold increase in ChREBP DNA-binding activity (FIG.6.7), and a 2.1 fold increase in transcript levels of $L P K$, a known target gene of ChREBP (FIG.6.8). All of these effects occurred at the $5 \mathrm{~h}$ time point but were reversed after long term $20 \mathrm{~h}$ anoxia exposure. Although $L P K$ gene expression probably also responds to other transcription factors, the correlation seen in turtle liver between ChREBP activation and the up-regulation of $L P K$ strongly suggests that $L P K$ expression is regulated in a ChREBP-dependent manner in response to anoxia. Furthermore, Brooks and Storey (1989) reported a 20\% increase in LPK maximal activity in liver of turtles subjected to $5 \mathrm{~h}$ anoxic submergence so it is possible that upregulation of the $L P K$ gene leads to increased LPK protein.

The response of turtles to oxygen deprivation during submergence occurs in two stages. In the initial stage, conditions become increasingly hypoxic, ATP levels fall somewhat and animals compensate by increasing glycolysis to meet organ demands for ATP. In contrast, when oxygen levels drop below a critical limit, a second phase is entered. Metabolic rate is strongly suppressed and this factor greatly lowers the demand for ATP by cellular processes resulting in energy conservation and a recovery of ATP levels (Kelly and Storey, 1988). Increased amounts of LPK within $5 \mathrm{~h}$ of anoxic 
submergence, which is within the hypoxic transition stage, could help hepatic cells enhance the glycolytic rate to meet cellular energy demands before the onset of metabolic rate depression. However, by $20 \mathrm{~h}$ of anoxia, the turtle is expected to significantly lower its metabolic rate, resulting in lower energy demands and making a high glycolytic rate (and therefore enhanced activation of the ChREBP pathway) unnecessary.

Previous studies have suggested that the initial response to submergence in $\mathrm{N}_{2}$ bubbled water (at $18^{\circ} \mathrm{C}$ ) is the Pasteur Effect, with glycolysis becoming activated in response to $1 \mathrm{~h}$ of anoxia in most organs (Kelly and Storey, 1988). By $5 \mathrm{~h}$, this was reversed and organs showed evidence of glycolytic inhibition. Applied to the present study which was carried out at $4{ }^{\circ} \mathrm{C}$, these periods of glycolytic activation and inhibition would be time-shifted and are represented by $5 \mathrm{~h}$ and $20 \mathrm{~h}$ anoxia exposures. Interestingly, the liver did not follow this pattern and showed evidence of glycolytic inhibition from $1 \mathrm{~h}$ (Kelly and Storey, 1988), a response that is quite typical when liver glycogenolysis is activated to produce glucose for export. However, it is possible that a Pasteur Effect does occur in the liver shortly after anoxic onset, with an initial influx in the glycolytic flux, followed by a decrease in the metabolic rate and export of glucose, concomitant with reduction in glycolysis. Overall, these results suggest a potentially important role for ChREBP in the hypoxic adaptation in turtle liver cells and induction of glycolytic genes by ChREBP could potentially be key to the animal's survival of hypoxic/anoxic onset in its initial hours.

Mechanistically, the activation of ChREBP in the turtle liver during anoxia is also expected. As oxygen runs out, the glycogen stores in the liver begin to break down in order to provide the animal with sufficient substrate to fuel glycolysis (Mehrani and 
Storey, 1985; Brooks and Storey, 1989). In consequence, the glucose content of the liver significantly increases (Buck et al., 1993). Since enhanced glucose content is the primary factor responsible for dephosphorylation and activation of ChREBP, these high glucose levels are probably responsible for nuclear translocation, as well as enhanced DNA-binding of ChREBP.

In contrast to the liver, the kidney presented a somewhat different activation pattern for ChREBP. Whereas ChREBP expression itself was elevated initially, with enhanced transcript and protein levels (1.2-1.9 fold, FIG.6.5) present in response to $5 \mathrm{~h}$ of anoxic submergence, as well as elevated amounts of ChREBP in the nucleus of $5 \mathrm{~h}$ anoxic animals (2.8 fold of control; FIG.6.6), no increase in ChREBP DNA-binding activity was observed (FIG.6.7). The upregulation of ChREBP transcript and protein levels during anoxia suggests that upstream mechanisms similar to those in the liver act to enhance ChREBP presence in the cell during short term anoxia, but ultimately, the pathway is attenuated. The differences in the ChREBP responses observed in the kidney and liver could potentially be attributed to the functional differences between the two tissues. While the liver does significantly decrease its metabolic rate in response to anoxia, it still continues to function as a central regulator of metabolism, whereas kidney function is greatly curtailed (Warburton and Jackson, 1995) and its energy demands might be different from those of the liver. It is also possible that some increase in kidney glycolytic flux does occur upon anoxic submergence, but that this increase is primarily mediated through changes in the properties of its regulatory enzymes, rather than an increase in their amounts. The attenuated ChREBP response in kidney and lack of change in ChREBP DNA binding could also be explained mechanistically. Since kidney does not 
have the high glycogen stores that the liver does, it is not expected to have as sharp an increase in glucose levels as observed for the liver in response to anoxia. The nuclear translocation in this case might not be due to enhanced glucose levels, but merely to the increased expression. Alternatively, it is possible that a mild increase in glucose levels does occur, sufficient to send ChREBP to the nucleus, but insufficient to acquire DNAbinding activity and activate gene transcription.

In summary, a strong activation of the transcription factor ChREBP was observed in the turtle liver in response to short-term anoxia. This activation was concomitant with enhanced levels of $L P K$ transcripts, suggesting that activation of ChREBP in response to anoxia might be contributing to elevated glycolytic flux, allowing hepatic cells to retain energy homeostasis during the hypoxic transition stage. Future studies could address other molecular mechanisms of potential relevance to metabolic adjustments during anoxia by, for example, examining transcription factors that regulate other important metabolic genes. 
FIG.6.1

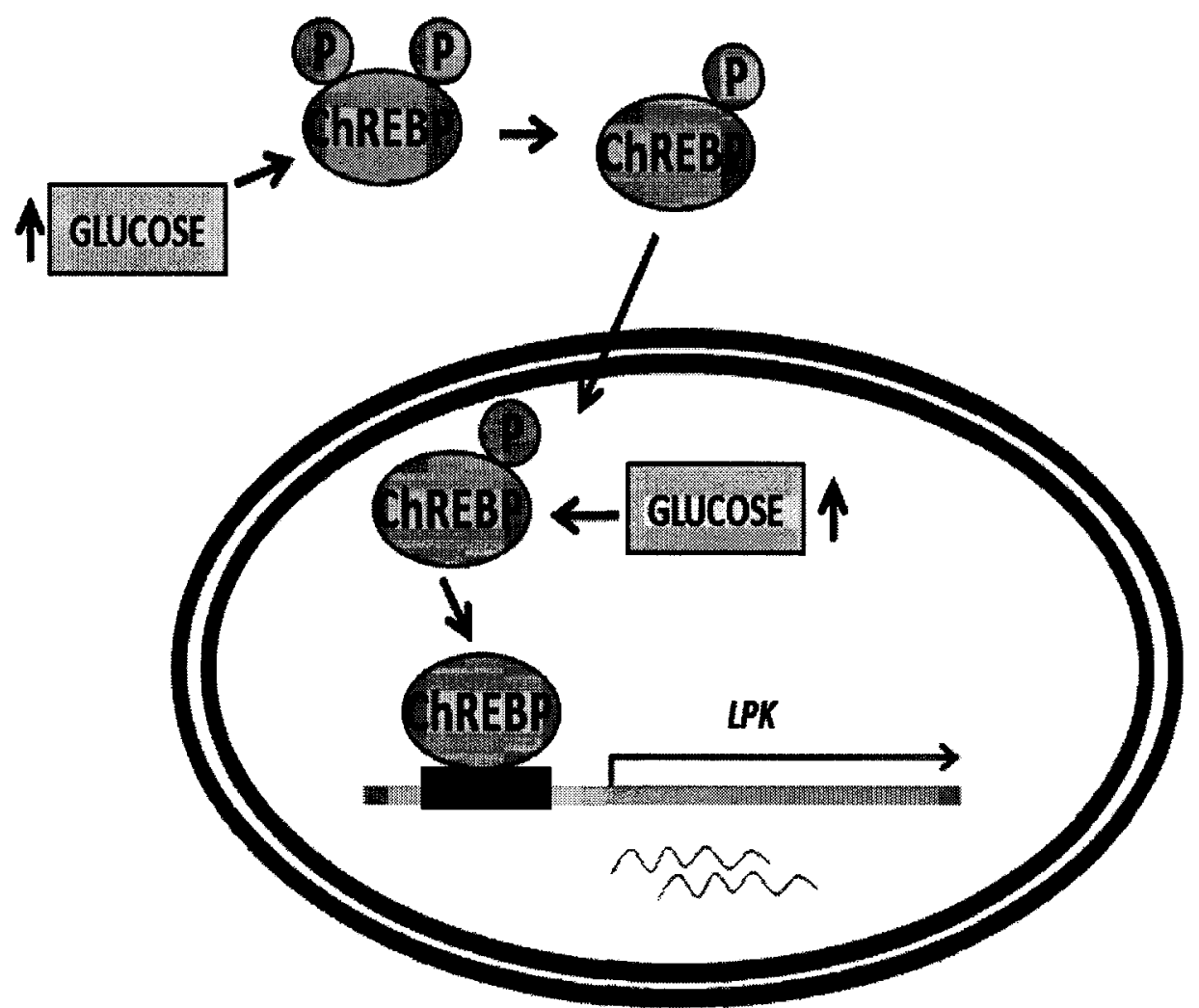

FIG.6.1. A model for ChREBP activation. Under basal conditions, ChREBP is found in the cytoplasm in a phosphorylated state. In response to high glucose, ChREBP becomes dephosphorylated and translocates to the nucleus. In the nucleus it is further dephosphorylated, allowing it to acquire DNA-binding activity. It then mediates the transcription of $L P K$ and other gene targets under its control. 
FIG.6.2

1

1

61

21

121

41

181

61

241

81

301

101

361

121

421

141

481

161

541

181

601

201
ACTCGGTGCCCGCCGGCGCCACCATGGTACTGAGCCGGAGCCAGCTGATCCCCACACCAT

$\begin{array}{llllllllllllllllllll}\mathrm{L} & \mathrm{G} & \mathrm{A} & \mathrm{R} & \mathrm{R} & \mathrm{R} & \mathrm{H} & \mathrm{H} & \mathrm{G} & \mathrm{T} & \mathrm{E} & \mathrm{P} & \mathrm{E} & \mathrm{P} & \mathrm{A} & \mathrm{D} & \mathrm{P} & \mathrm{H} & \mathrm{T} & \mathrm{I}\end{array}$

CGACCTGACCCTCACTCGGCTCTTCGAGTGCATGAGCCTGGCCTACAGTGGGAAGCTGGT

$\begin{array}{llllllllllllllllllll}\mathrm{D} & \mathrm{L} & \mathrm{T} & \mathrm{L} & \mathrm{T} & \mathrm{R} & \mathrm{L} & \mathrm{F} & \mathrm{E} & \mathrm{C} & \mathrm{M} & \mathrm{S} & \mathrm{L} & \mathrm{A} & \mathrm{Y} & \mathrm{S} & \mathrm{G} & \mathrm{K} & \mathrm{L} & \mathrm{V}\end{array}$

GTCTCCGAAATGGAAGAATTTCAAGGGTCTGAGGCTGCTTTGCAGGGATAAAATCCGCCT $\begin{array}{llllllllllllllllllll}\mathrm{S} & \mathrm{P} & \mathrm{K} & \mathrm{W} & \mathrm{K} & \mathrm{N} & \mathrm{F} & \mathrm{K} & \mathrm{G} & \mathrm{L} & \mathrm{R} & \mathrm{L} & \mathrm{L} & \mathrm{C} & \mathrm{R} & \mathrm{D} & \mathrm{K} & \mathrm{I} & \mathrm{R} & \mathrm{L}\end{array}$

CAACAATGCTATCTGGAGAGCCTGGTACATCCAATATGTGGAGCGGAGGAAGAGCCCCGT $\begin{array}{llllllllllllllllllll}N & N & A & I & W & R & A & W & Y & I & Q & Y & V & E & R & R & K & S & P & V\end{array}$

GTGTGGGTTTGTGACCCCCCTGGAGGGCTCCGAGGCGGAAGAGCACCGGAAACCCGAGGC $\begin{array}{llllllllllllllllllll}C & G & F & V & T & P & L & E & G & S & E & A & E & E & H & R & K & P & E & A\end{array}$ TGTTGTGTTGGAGGGGAACTACTGGAAGCGACGTATCGAGGTGGTGATGAAGGAGTATCA $\begin{array}{llllllllllllllllllll}\mathrm{V} & \mathrm{V} & \mathrm{L} & \mathrm{E} & \mathrm{G} & \mathrm{N} & \mathrm{Y} & \mathrm{W} & \mathrm{K} & \mathrm{R} & \mathrm{R} & \mathrm{I} & \mathrm{E} & \mathrm{V} & \mathrm{V} & \mathrm{M} & \mathrm{K} & \mathrm{E} & \mathrm{Y} & \mathrm{H}\end{array}$ CAAGTGGAGGATTTACTACAAGAAGCGGCTTCGAAAGTCCAGCCGGGAGGGTGAGATTTC $\begin{array}{llllllllllllllllllll}K & \text { W } & R & I & Y & Y & K & K & R & \text { L } & R & K & S & S & R & E & G & E & I & S\end{array}$ CAGCCCGAAGCAGGATGAGGGTGTGTGGAAGCCATCGGAGAAATGGTGCAACCAGCTATT $\begin{array}{llllllllllllllllllll}S & P & K & Q & D & E & G & V & W & K & P & S & E & K & W & C & N & Q & L & F\end{array}$ CTCCAGCGTTGTGCCCATGCTGCTGGGGGATGAGAATGAGGAGGCCGGAGGGCGGCAGCT $\begin{array}{llllllllllllllllllll}S & S & V & V & P & M & \text { L } & \text { L } & G & \text { D } & \text { E } & \text { N } & \text { E } & \text { E } & \text { A } & \text { G } & \text { G } & \text { R } & \text { Q } & \text { L }\end{array}$ CTTTGACTTGGACAGCTTCCTCTCGGACATCTCAGACACACTCTTCACCATGACGCAGCC $\begin{array}{lllllllllllllllllllll}F & D & L & D & S & F & L & S & D & I & S & D & T & L & F & T & M & T & Q & P\end{array}$ CCCCTGCTCCCACCAGACGCTCCCAGAAGACGCCTACACTGGGAACGC

$\begin{array}{lllllllllllllll}P & C & S & H & Q & T & L & P & E & D & A & Y & T & G & N\end{array}$

FIG. 6.2. Nucleotide and deduced amino acid sequence for $T$. s. elegans partial ChREBP sequence. Nucleotides and amino acids are numbered on the left. The nucleotide sequence was 648 nucleotides in length, while the amino acid sequence was 215 amino acids. This protein covered amino acids $68-278$ of the chicken ChREBP sequence, representing $\sim 25 \%$ of the total ChREBP protein. 
FIG.6.3

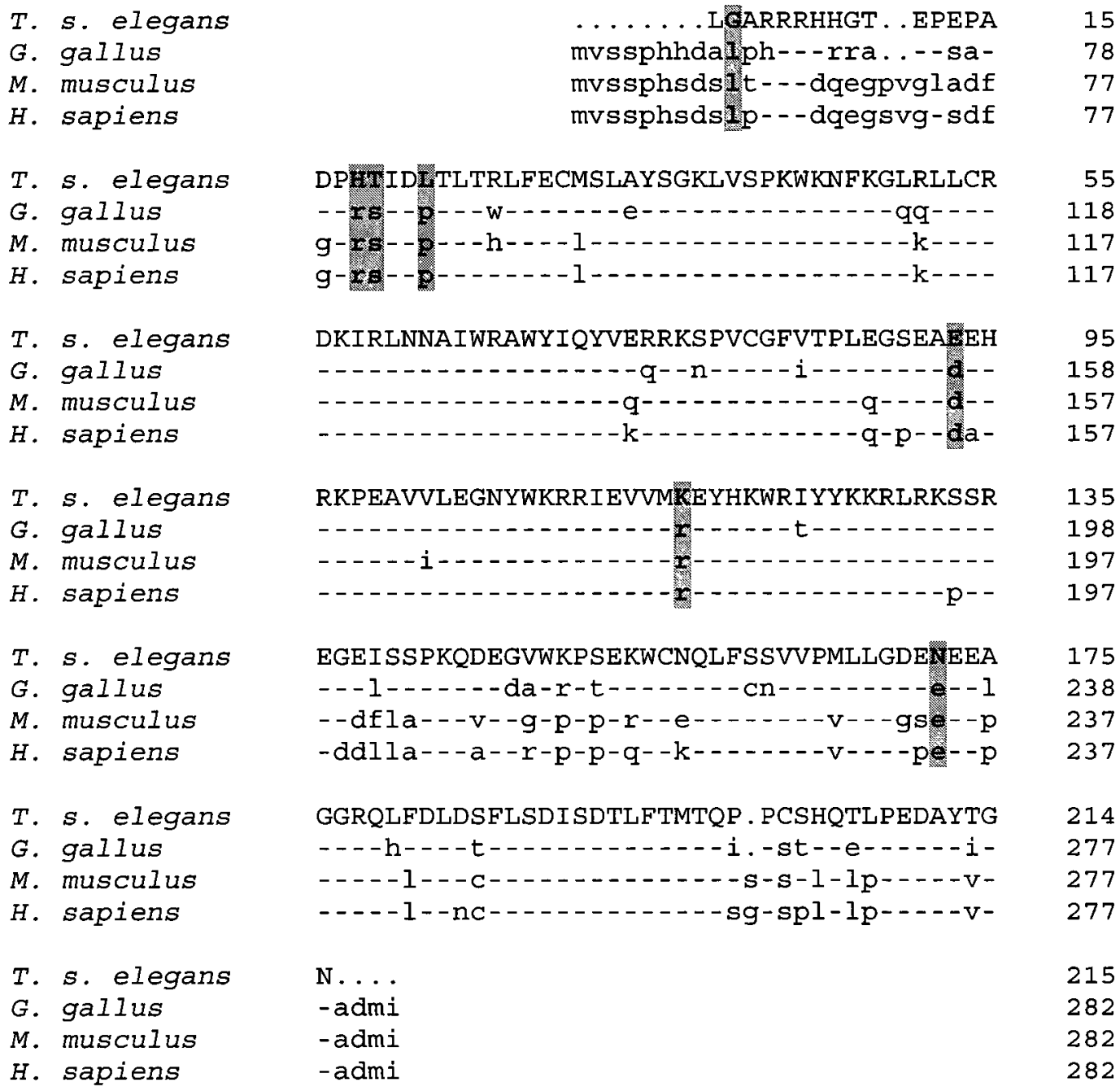

FIG. 6.3. The partial amino acid sequence of ChREBP from $T$. s. elegans aligned with ChREBP sequences from chicken (Gallus gallus), human (Homo sapiens) and mouse (Mus musculus). Accession numbers are NP_001104311, NP_116569.1 and NP_067430.2 for human, chicken and mouse, respectively. Dashes replace amino acid residues identical with $T$. $s$. elegans whereas spacer dots indicate residues that are missing in the sequence. Turtle-specific amino acid substitutions are bolded and shaded. 


\section{FIG.6.4}

A)
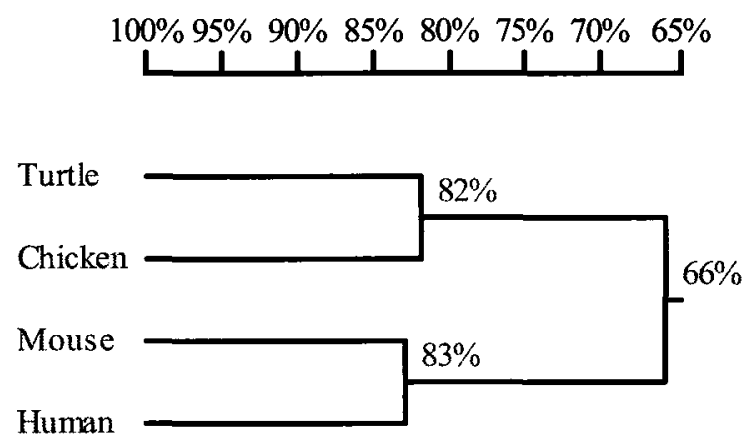

B)

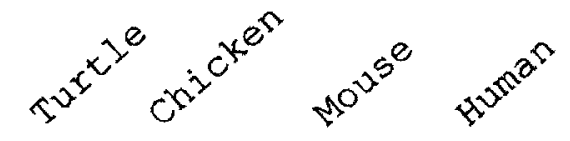

Turtle
Chicken
Mouse
Human

$100 \%$

$82.3 \frac{0}{6} 100 \%$

$78.6 \% 51.6$ 응 $100 \%$

$77.2 \% \quad 55.2 \% \quad 82.6$ 응 $100 \%$

FIG. 6.4. Homology tree and matrix for T. s. elegans ChREBP compared with other species

(A) Homology tree produced from an alignment of the T. s. elegans ChREBP protein sequence with ChREBP from chicken (Gallus gallus), human (Homo sapiens) and mouse (Mus musculus). Accessions numbers are the same as the previous figure.

(B) Homology matrix showing amino acid identity of the $T$. $s$. elegans ChREBP sequence compared with ChREBP from the other vertebrates. 
FIG. 6.5
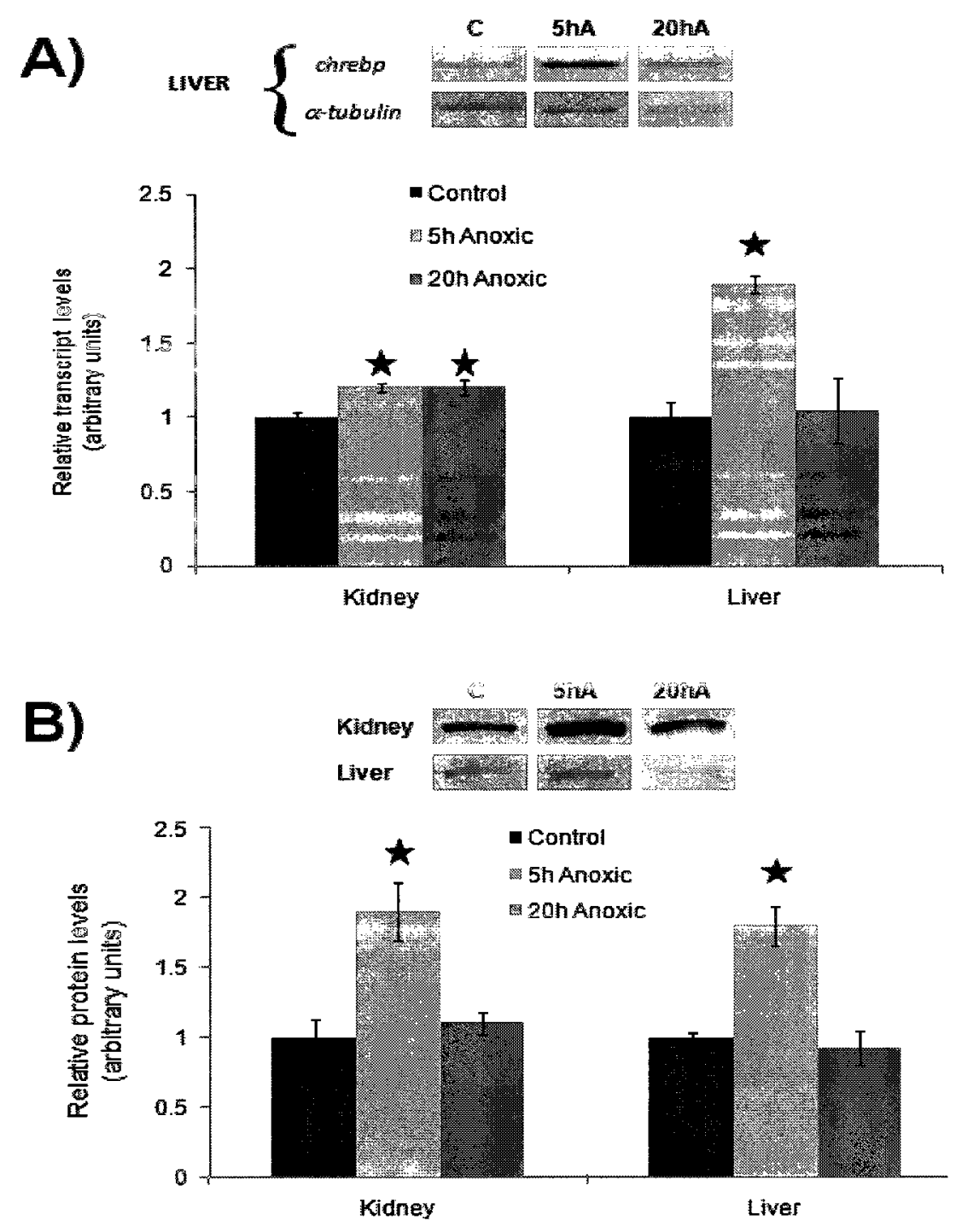

FIG. 6.5. Effect of 5 or $20 \mathrm{~h}$ of anoxic submergence on ChREBP expression in $T$. $s$. elegans kidney and liver.

(A) Changes in chrebp transcript levels in response to anoxia, as determined by RT-PCR. Bands show representative chrebp amplicons for liver with corresponding $\alpha$-tubulin for control and anoxic conditions. The histogram shows the normalized transcript levels under control and anoxic conditions for liver and kidney; bands were normalized against the corresponding $\alpha$-tubulin bands amplified from the same sample.

(B) Changes in ChREBP protein levels in response to anoxia, as determined by Western immunoblotting. Representative western immunoblot bands are shown above the histogram. The histogram shows normalized protein levels under control and anoxic conditions.

Data are means \pm SEM, $n=3-5$ independent trials. * Significantly different from the corresponding control, $P<0.05$. 
FIG. 6.6

A)

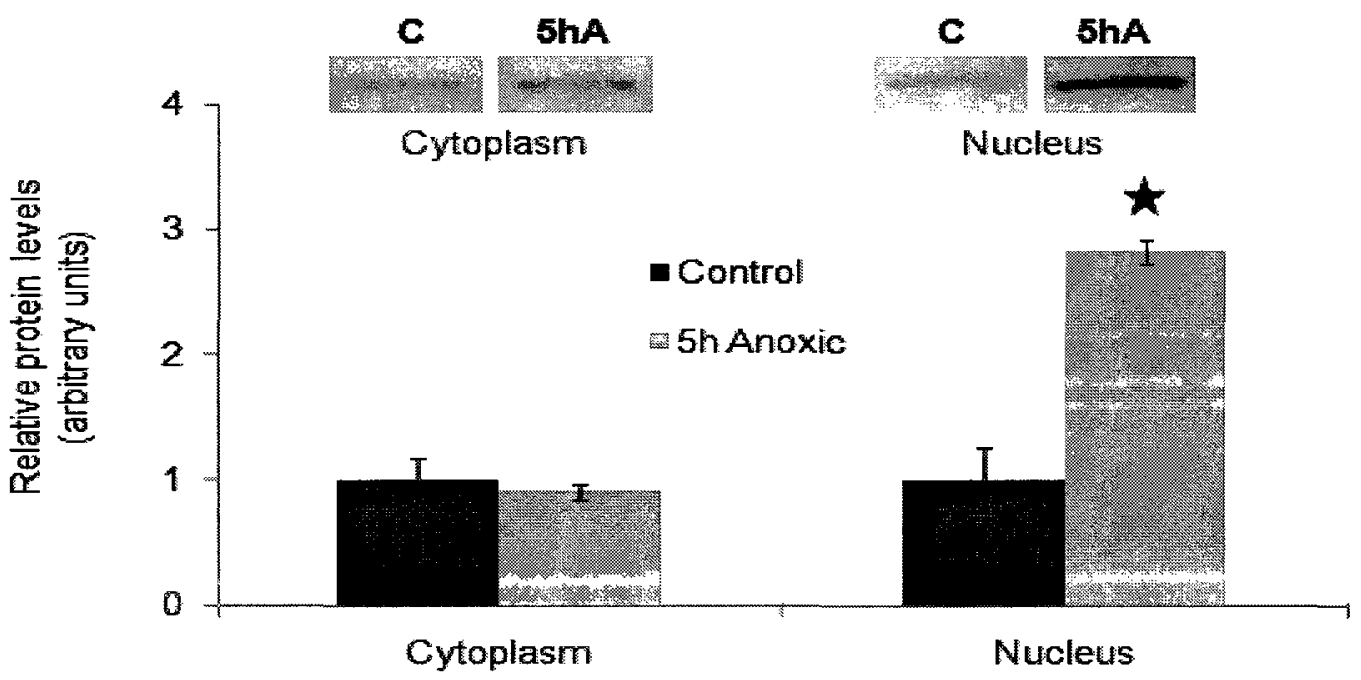

B)

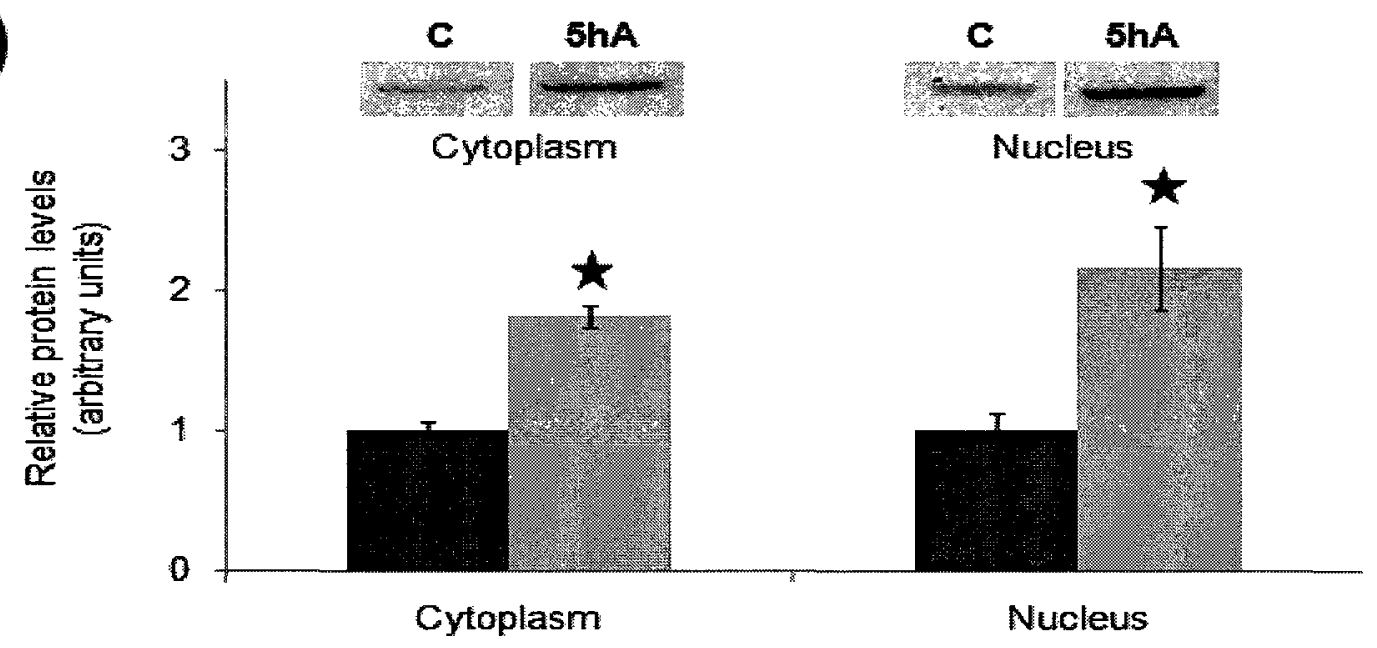

FIG. 6.6. Effect of $5 \mathrm{~h}$ anoxic submergence on subcellular distribution of ChREBP. Changes in ChREBP protein levels in kidney (A) and liver (B) cytoplasm and nucleus were determined by Western immunoblotting. Representative immunoblot bands are shown above the histogram. Data are means \pm SEM, $n=3-4$ independent trials. * Significantly different from the corresponding control, $P<0.05$. 
FIG. 6.7

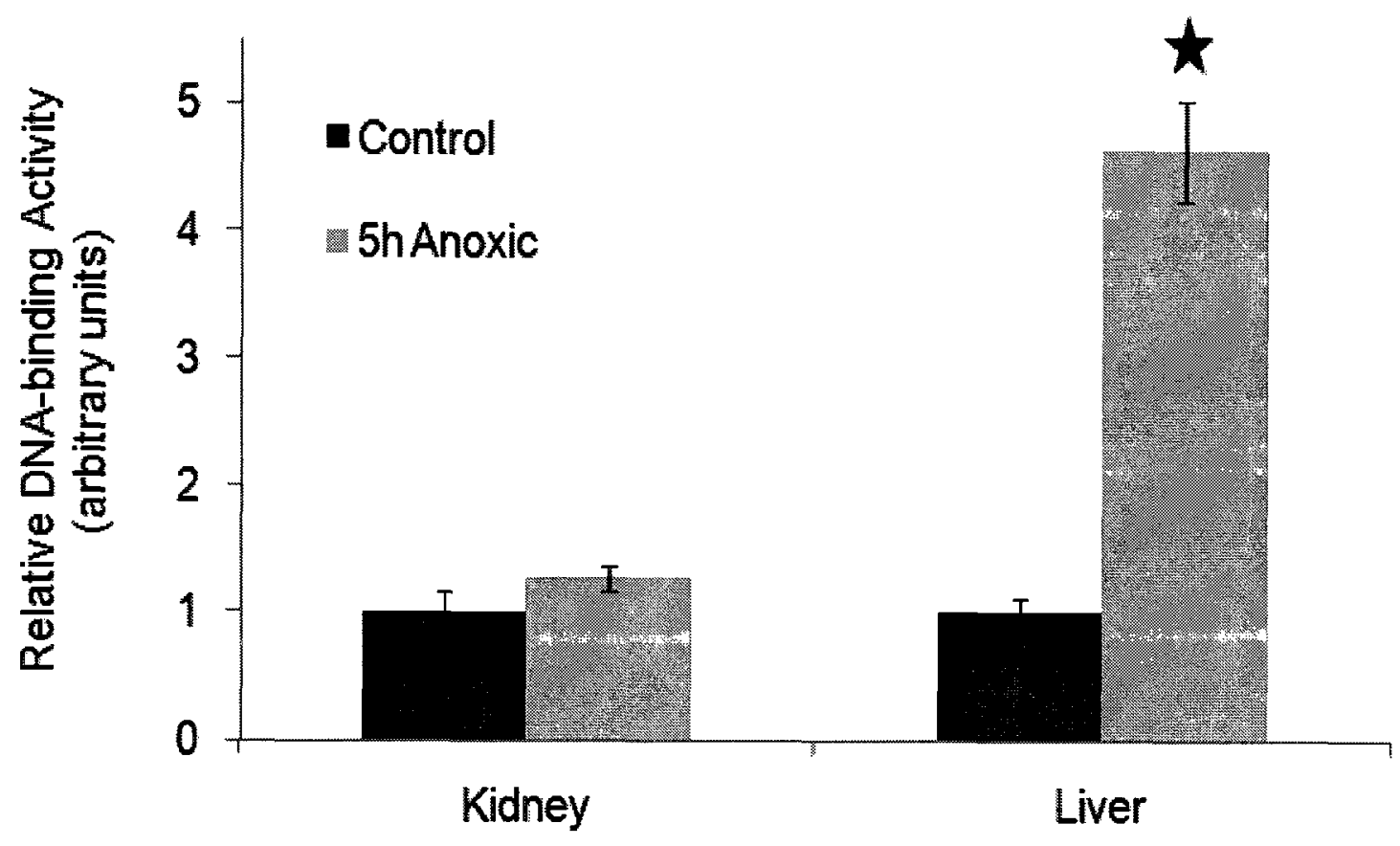

FIG. 6.7. Effect of $5 \mathrm{~h}$ anoxic submergence on the DNA-binding activity of ChREBP in nuclei isolated from kidney and liver as determined by a DNA-binding assay. Data are means \pm SEM, $n=3-4$ independent trials for each tissue. * Significantly different from the corresponding control, $P<0.05$. 
FIG. 6.8

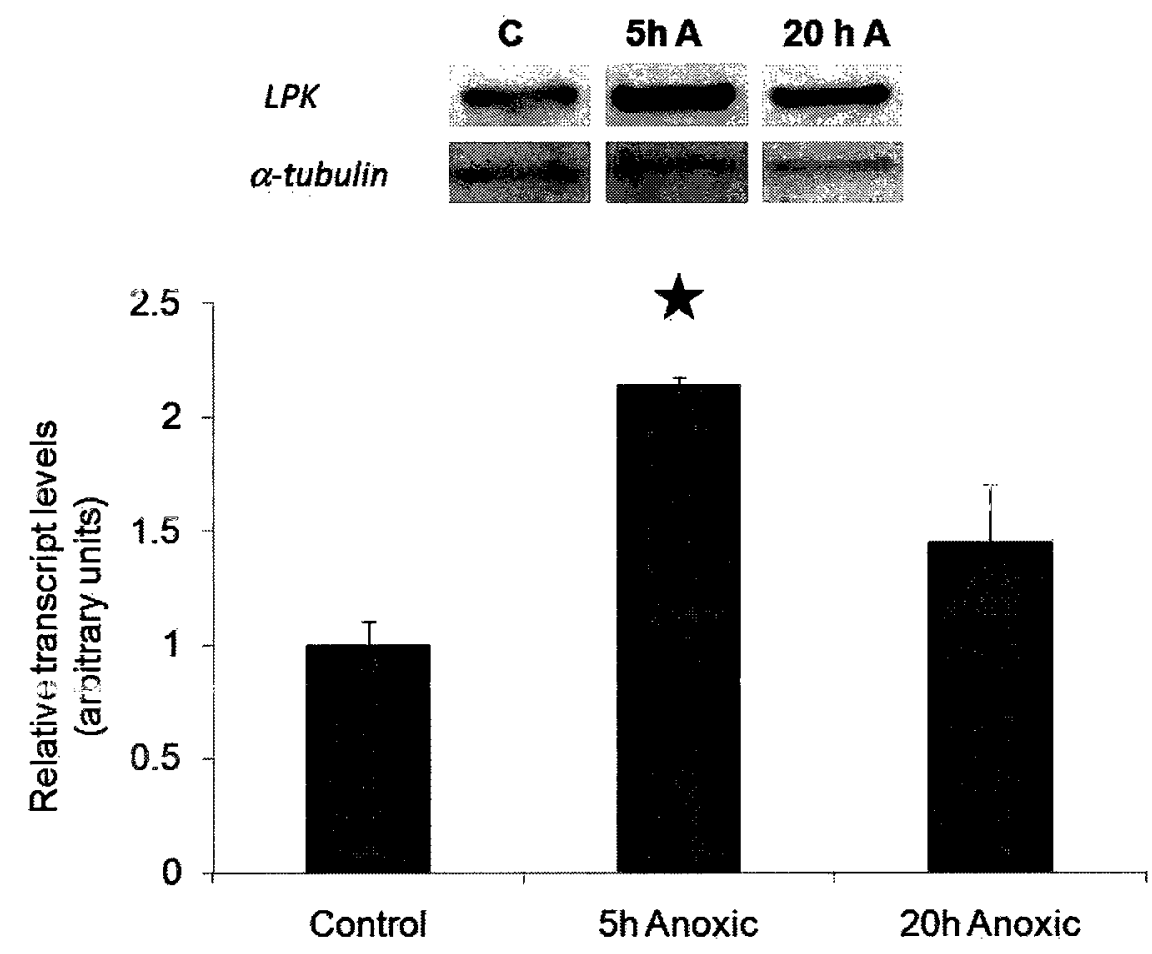

FIG. 6.8. Effect of 5 or $20 \mathrm{~h}$ anoxic submergence on $L P K$ transcript expression in $T$. s. elegans liver as determined by RT-PCR. Bands show representative $l p k$ amplicons with corresponding $\alpha$-tubulin for control and anoxic conditions. The histogram shows normalized transcript levels under control and anoxic conditions; bands were normalized against the corresponding $\alpha$-tubulin bands from the same sample. Data are means \pm SEM, $n=3-4$ independent trials. * Significantly different from the corresponding control, $P<$ 0.05 . 


\section{CHAPTER 7}

\section{General Discussion}


Oxygen deprivation is extremely harmful for most organisms. In aerobic animals, the complete catabolism of all fuel sources relies on oxidative phosphorylation, in which oxygen plays a crucial role as the final electron acceptor. When oxidative phosphorylation is inhibited, anaerobic glycolysis becomes the sole energy producer of the cell. However, this pathway has several limitations, including poor ATP yield, toxic end products (lactic acid), and a quick depletion of fuel sources. Therefore, most aerobic life forms are fairly intolerant of oxygen limitation and sustain significant damage and/or death when subjected to anoxia.

In contrast, some animals can survive quite well with or without oxygen. Among terrestrial vertebrates, the best facultative anaerobes are turtles belonging to the Trachemys and Chrysemys genera, including the red eared slider, Trachemys scripta elegans and the painted turtle Chrysemys picta. These turtles have developed several mechanisms to deal with the problems posed by usage of anaerobic glycolysis as their primary ATP producing pathway (Storey, 2007). The end product of glycolysis, lactate, is collected and stored in the shell and bones of the turtles, and $\mathrm{H}^{+}$is neutralized by bicarbonate released from shell and bone, preventing acidification. In addition, huge glycogen stores in the liver ensure adequate fuel to supply glycolysis for extended periods of anaerobic life. Finally, to deal with the problem of insufficient ATP production by glycolysis, turtles significantly depress their metabolic rate to $10-20 \%$ of the corresponding aerobic rate, as well as reorganize their metabolic priorities, in order to meet the energy needs of vital cell functions in the anoxic state (Storey, 1996, 2007).

While research on turtle anoxia tolerance has primarily been focused on the physiological and biochemical processes involved, little has been done to investigate the 
gene expression responses to anoxia in turtles. The goal of my thesis was to address this problem by using $T$. s. elegans as the model and examining the transcriptional responses to three key aspects of anoxia survival: stress-tolerance, hypometabolism and metabolic reorganization. I used a variety of different techniques, including western immunoblotting, subcellular fractionation, semi-quantitative RT-PCR and Transcription Factor ELISAs to examine the responses to anoxia of several important proteins and pathways at various levels of regulation, including transcript expression, protein levels, subcellular localization, post-translational modifications and DNA-binding activity. The results of my thesis point to multiple previously unknown mechanisms that play a role in anoxia tolerance.

\section{ANOXIA TOLERANCE AND THE STRESS RESPONSE}

Complete oxygen deprivation can present several problems to the cell. Besides the aforementioned stress on metabolism, oxygen deprivation is characterized by an altered redox environment, as well as decreased cellular $\mathrm{pH}$ resulting from lactic acid production. These conditions could potentially be stressful to the cell and compromise various cellular processes, such as protein folding. Furthermore, when animals use metabolic rate depression to sustain viability over long periods of stress, the strong suppression of ATPexpensive activities such as transcription, translation, and protein degradation means that cells must place a greater emphasis on extending the functional lifespan of existing proteins because the option of protein turnover is very much reduced. Therefore, an important aspect of anoxia tolerance might involve the induction of various cytoprotective pathways that could ensure a smooth transition into and maintenance of the anoxic state. In my thesis, I examined several pathways and proteins (primarily in 
Chapters 2 and 3) that could contribute to stress tolerance in T. s. elegans.

\section{Protein folding and the unfolded protein response}

As mentioned above, long term maintenance of native protein structure could be compromised in the anoxic state and protective mechanisms might be required to ensure correct folding. Indeed, previous studies in turtle tissues have shown that various molecular chaperones are induced in response to anoxia (Kesaraju et al., 2009; Prentice et al., 2004; Ramaglia and Buck, 2004; Krivoruchko and Storey, 2010b; Storey, 2007), suggesting that folding stress does occur. In my thesis, I investigated this matter further by examining a key pathway associated with folding stress: the unfolded protein response (UPR). The endoplasmic reticulum (ER) is the site of secretory protein synthesis as well as the site where the majority of secretory and transmembrane proteins are folded into their native conformations and undergo post-translational modifications. Various stresses can compromise the folding functions of the ER resulting in accumulation of unfolded proteins in the ER lumen and leading to activation of the UPR (Schroder, 2008; Schroder and Kaufman, 2005a,b). This response is composed of several branches controlled by three main ER-resident sensors and is activated with the purpose of eliminating folding stress by (temporarily) reducing/ceasing protein translation, degrading permanently misfolded or slowly folding proteins, and inducing various molecular chaperones to enhance the folding capacity of the cell (Schroder, 2008; Schroder and Kaufman, 2005a,b).

Chapter 2 examines the activation of the UPR in turtle tissues with an emphasis on the PERK-controlled branch of this pathway. PERK is a transmembrane ER protein 
that becomes activated through phosphorylation in response to ER stress. Upon activation, it phosphorylates the $\alpha$ subunit of eukaryotic initiation factor 2 , inhibiting capdependent translation (Harding et al, 1999; Shi et al, 1998; Kaufman, 2004). Chapter 2 showed that the phosphorylated levels of PERK increased in the heart, kidney, liver and muscle of turtles in response to anoxia (FIG.2.2A), suggesting that the UPR is activated in response to anoxic stress. In addition, increased phosphorylation of eIF2 $\alpha$ was observed in the heart, kidney and liver (FIG.2.2B), indicating a UPR-mediated inhibition of protein translation in these tissues in response to anoxia. This translational inhibition could decrease the number of unfolded proteins that accumulate in the ER lumen during anoxia, alleviating ER stress, as well as contribute to the general lowering in protein synthesis rates observed in these tissues during anoxia.

The transcription factor ATF4, which lies downstream of PERK activation, was also examined in Chapter 2. This transcription factor is regulated by a unique mechanism that suppresses translation under normal conditions, but induces translation under stress in response to eIF $2 \alpha$ phosphorylation. This occurs because of the presence of multiple upstream reading frames (uORFs) present 5' of its reading frame (Wek and Cavener, 2007). In Chapter 2, I cloned the full-length sequence of ATF4 mRNA from T. s. elegans and found that it contained the same regulatory upstream structure as found in other vertebrates (FIG.2.3), suggesting that it is regulated in a similar fashion under stress conditions. ATF4 was activated in response to anoxia in heart, kidney and liver, as determined through measurement of its levels, nuclear presence, and DNA-binding activity (FIG.2.6 and 2.7). ATF4 plays important roles in induction of various protective proteins including chaperones, as well as antioxidant defenses, and its anoxia-mediated 
activation in turtle tissues underlines the importance of this transcription factor in protecting these tissues during anoxic stress.

In addition to ATF4 activation, upregulation of one of its targets, ATF3, was observed in heart and liver of anoxia-exposed turtles (FIG.2.9). While the exact functions of this transcription factor are not well-known, it is generally characterized as an adaptive-response protein (Hai, 2006; Lu et al., 2006) that is inducible by a variety of stressful conditions (Hai, 2006). ATF3 appears to be involved in the induction of a program of gene expression responsible for cellular remediation, as well as apoptosis (Harding et al, 2003) and its activation in the heart and kidney suggests a potential cytoprotective role in these tissues. In addition, ATF3 has been shown to contribute to the expression of GADD34 (Jiang et al., 2004), and its anoxia-responsive expression pattern does appear to correlate with that of GADD34 (FIG.2.10), suggesting that GADD34 could be activated in an ATF3-dependent manner in response to anoxia. GADD34 is required for feedback inhibition of the UPR by targeting the type 1 serine/threonine protein phosphatase to eIF2 $\alpha$ and dephosphorylating it (Jiang et al, 2004; Connor et al, 2001; Brush et al, 2003), and GADD34 could potentially play a similar role in turtle heart tissue. In the liver, however, GADD34 upregulation in response to $5 \mathrm{~h}$ of anoxia did not match the observation that phosphorylation of eIF $2 \alpha$ was still elevated at $20 \mathrm{~h}$ of anoxia, suggesting that GADD34 plays a different role in this tissue. For example, some studies have shown increased GADD34 expression to be associated with increased expression of the cell cycle inhibitor p21 (Minami et al., 2007; Yagi et al., 2003), which could contribute the overall metabolic depression that characterizes anoxia. Indeed, such an activation pattern in the liver seems to be in concert with data from Chapters 4 and 5 , 
that show that other proteins implicated in cell cycle arrest are upregulated under anoxia.

I also measured the anoxia-responsive expression of two other UPR target genes, GRP78 and GRP94 (FIG.2.12 and 2.13). Both are important ER chaperones that function in the folding of protein substrates and are inducible by multiple UPR branches (Lee, 2001). These proteins were also induced in response to anoxia, GRP78 being upregulated in the heart, kidney and liver, and GRP94 in heart and liver. Since GRP94 is not a known ATF4 target, its upregulation in response to anoxia hints at the possible activation of additional UPR branches.

Overall, the data in Chapter 2 suggest that the UPR is a key component of anoxia survival in multiple turtle tissues and activation of this pathway could contribute to a safe transition into the anoxic state.

$N F-\kappa B$

Chapter 3 of my thesis explored the activation of NF- $\kappa B$, another important stress-responsive transcription factor. NF- $\mathrm{KB}$ is an oxygen-responsive transcription factor that is known to be activated by a variety of stimuli and control the expression of hundreds of target genes, including genes involved in the immune response, stress response, antioxidant defenses, cell growth and differentiation, and apoptosis (reviewed by Pahl, 1999). NF- $\kappa B$ is a dimeric transcription factor and is composed of proteins containing the Rel Homology domain. The best studied dimer, and the one typically referred to as 'NF- $\mathrm{KB}$ ' occurs between p65 (RelA) and p50. Under normoxic conditions $\mathrm{NF}-\kappa \mathrm{B}$ is retained in the cytoplasm through association with the inhibitor I $\mathrm{KB}$, but in response to anoxia, it undergoes nuclear translocation, mediating the expression of target 
genes. This control mechanism allows NF- $\mathrm{kB}$ to respond rapidly to stress. In mammals, $\mathrm{NF}-\mathrm{KB}$ acts as an important mediator of transcriptional responses under ischemia/ reperfusion (reviewed in by Latanich and Toledo-Pereyra, 2009). Chapter 3 shows that activation of NF- $\mathrm{kB}$ occurs in T. s. elegans liver in response to anoxia. The phosphorylation status of the NF- $\mathrm{kB}$ inhibitor rose in response to $5 \mathrm{~h}$ anoxia, suggesting NF- $\mathrm{kB}$ activation (FIG. 3.2), increased expression of $\mathrm{p} 50$ and $\mathrm{p} 65$ was observed, and nuclear enrichment and increased DNA-binding activity occurred (FIG.3.5, 3.6, 3.7 and 3.8). These data suggest that NF- $\mathrm{kB}$ has key functions in protecting the turtle liver under anoxic conditions. Whereas many of the genes inducible by NF- $\mathrm{kB}$ could play beneficial roles in anoxia tolerance, the vast array of genes that lie under NF- $\mathrm{KB}$ control, as well as the lack of tools available to accurately analyze large-scale transcriptional responses in the turtle, makes it rather difficult to pinpoint all the genes that are activated by NF- $\mathrm{kB}$ in turtle liver in response to anoxia. Some potential candidates include the antioxidant genes, ferritin heavy chain and SOD, as well as the pro-survival genes Bcl-2 and Bcl-xL. Indeed, my data from Chapter 3 show increased transcript levels of these genes in anoxic turtle liver that could lead to a corresponding increase in their protein levels.

\section{$N F-\kappa B$ and antioxidant proteins}

The electron carriers of the mitochondrial transport chain become reduced under anoxia, which can potentially lead to increased production of reactive oxygen species (ROS) upon reoxygenation (Hermes-Lima et al., 2001), resulting in oxidative stress. In mammalian systems undergoing ischemia and reperfusion, the reperfusion phase can be just as dangerous as the ischemic phase, since many organs can incur oxidative damage 
during this phase (Bolli and Marban, 1999; Lipton, 1999). Anoxia-tolerant animals solve this problem by both maintaining high constitutive levels of antioxidant defenses, as well as upregulating antioxidant proteins in preparation for recovery (Bolli and Marban, 1999; Lipton, 1999). Previous research showed that turtles used the former mechanism, displaying constitutively high activities of antioxidant enzymes (Willmore and Storey, 1997a,b). My results from Chapter 3 indicate that turtles use the latter mechanism as well, because anoxia exposure upregulated transcript levels of $\mathrm{Cu} / \mathrm{Zn}$ SOD, Mn SOD, and ferritin (FIG.3.12). Ferritin sequesters redox active iron, thereby reducing the potential hydroxyl radical formation via the Fenton reaction (Hentze et al., 2004). $\mathrm{Cu} / \mathrm{Zn}$ - and $\mathrm{Mn}$ SOD reduce oxidative stress by catalyzing the dismutation of superoxide radicals into water and hydrogen peroxide. All of these proteins lie downstream of NF- $\mathrm{kB}$ activation and their upregulation in the liver in response to anoxia could be due to NF- $\mathrm{kB}$ action.

Interestingly, in addition to the increases in the transcript levels of the enzymes mentioned above, Chapter 4 of my thesis also showed that transcript levels of another antioxidant enzyme, catalase, also increased in turtle liver in response to $5 \mathrm{~h}$ anoxia (FIG.4.13). This enzyme is an important component of the cellular antioxidant defenses and functions in reducing $\mathrm{H}_{2} \mathrm{O}_{2}$ to oxygen and water. Together, these results suggest an important role for cellular antioxidant defenses in the liver in response to anoxia.

\section{Anti-apoptotic proteins}

Mammalian studies have shown that apoptosis is oxygen-responsive, with exposure to hypoxia promoting cell survival and exposure to anoxia promoting apoptosis 
(Santore et al., 2002; Papandreou et al., 2005). However, tissues of $T$. s. elegans can survive anoxia for many weeks without any apparent damage, suggesting that inhibition of apoptosis must occur on a molecular level during anaerobiosis. The promotion or inhibition of apoptosis is controlled by two conserved pathways, the death receptor pathway and the mitochondrial pathway, and the decision to initiate apoptosis is often dependent on the levels of pro- and anti-apoptotic proteins (Gupta, 2001). The transcript levels of two major anti-apoptotic proteins, Bcl-2 and Bcl-xL, were analyzed in Chapter 3 and were shown to increase (FIG.3.14), possibly in an NF-אB-dependent manner, in response to anoxia in turtle liver. These proteins reside in the outer mitochondrial membrane and inhibit apoptosis by preventing the loss of outer mitochondrial membrane integrity, which leads to lethal downstream events such as activation of caspases (Kluck et al., 1997; Janumyan et al., 2003). Since previous studies, as well as my results in Chapters 2 and 3, suggest that the turtle liver does experience stress in response to anoxia, these stressful stimuli could potentially promote apoptotic responses. Increased apoptosis during anoxia could be quite damaging, since it could lead to tissue loss, and therefore upregulation of anti-apoptotic proteins during the anoxic episode would counteract any apoptotic signals and ensure tissue integrity. The upregulation of these proteins further highlights the importance of protecting the liver against anoxia-mediated stress.

\section{ANOXIA TOLERANCE AND HYPOMETABOLISM}

Because anaerobic glycolysis is not nearly as efficient as oxidative phosphorylation at producing ATP, cellular energy requirements must be lowered during 
periods of anaerobiosis so that they can be met by anaerobic energy supply. This involves a coordinated downregulation of many energy-consuming processes, including gene transcription, protein translation and ion motive ATPases, as well as a reorganization of cellular priorities (Storey, 1996, 2007). In my research, I decided to examine molecular mechanisms that could promote metabolic rate depression in turtle cells, and by doing so promote survival under anoxic conditions.

\section{Hypometabolism and FoxOs}

In Chapter 4, I examined the anoxia-responsive activation of FoxOs, a group of conserved transcription factors that have been implicated in hypometabolism. Their hypometabolic roles have been largely studied in C. elegans, where the absence of Akt signaling results in the activation of the FoxO homologue, DAF-16, and subsequent entrance into the quiescent dauer state (Ogg et al., 1997; Paradis et al., 1998). Activation of FoxOs is largely controlled in a negative manner by Akt-mediated phosphorylation, where increased phosphorylation of FoxO members results in their nuclear exclusion, whereas reduced phosphorylation levels leads to FoxO activation (Rena et al., 2001; Brownawell et al., 2001; Brunet et al., 2002).

Chapter 4 examined the expression and activation of FoxO1 and FoxO3, as well as several target genes, in response to anoxic submergence in T. s. elegans. FoxO1 was shown to be activated in liver, as evident through increased transcript/protein levels, decreased phosphorylation and increased nuclear levels and DNA-binding activity (FIG. 4.4, 4.5 and 4.6). FoxO3 was activated in heart, kidney, and liver as shown by increased nuclear localization and increased DNA-binding activity (FIG.4.9, 4.10 and 4.11). 
Interestingly, enhanced nuclear localization and subsequent activation was sometimes observed for FoxO3 despite unchanged or even elevated phosphorylation state in response to anoxia, suggesting that some additional factors may also regulate FoxO activity. Stress stimuli have been shown to trigger FoxO activation regardless of their phosphorylation status (Brunet et al., 2004; Kitamura et al., 2005; Frescas et al., 2005). Chapter 2 suggested that cell stress occurred in heart, kidney and liver and FoxO3 activation in these tissues might therefore be stress-mediated.

In some instances, such as in the case of FoxO3 in the heart, as well as both FoxOs in kidney and muscle, increased protein expression was observed despite constant transcript levels. This observation suggests that a nontranscriptional mode of FoxO regulation contributes to the anoxia response, possibly control of FoxO degradation by polyubiquitination, that had been shown previously to be an effective part of FoxO control (Matsuzaki et al., 2003; Plas and Thompson, 2003; Kitamura et al., 2005).

While Chapter 4 showed that FoxOs were activated in heart and kidney under anoxia, the liver is the main organ where FoxO hypometabolic actions in cell cycle arrest would be expected to occur. Indeed, transcript levels of p27kipl, an important FoxOcontrolled cell cycle inhibitor, were anoxia-inducible in turtle liver (FIG.4.12), suggesting a FoxO-mediated inhibition of the cell cycle.

It should be noted that despite increased FoxO activation in the heart and kidney, no increase in any of the target genes examined was observed in these tissues. A possible explanation is that other FoxO target genes become activated in response to anoxia in these tissues, and these should be examined further.

Overall, the data presented in Chapter 4 indicated a strong activation of FoxOs in 
the liver, and to a lesser extent in the heart and kidney, suggesting an important role for these transcription factors in turtle tissues in response to anoxia, and a potentially important role in mediating cell cycle arrest in the liver.

\section{Hypometabolism and epigenetics}

In Chapter 5, I examined another mechanism that could potentially contribute to hypometabolism in turtle tissues: epigenetic modifications. It is estimated that $1-10 \%$ of cellular energy is devoted to gene transcription, depending on the tissue (Rolfe and Brown, 1997), a significant metabolic cost that must be reduced in situations of hypometabolism. Suppressed rates of gene transcription have been observed in a number of anoxia-responsive hypometabolic systems including brine shrimp (Artemia franciscana) and intertidal snails (Littorina littorea) (Van Breukelen et al., 2000; Larade and Storey, 2002), as well as in states of aerobic hypometabolism such as mammalian hibernation (Bocharova et al., 1992; Van Breukelen and Martin, 2002). A prominent mechanism of global or localized transcriptional control is the posttranslational modification of histones (Allfrey et al., 1964; Spotswood and Turner, 2002). Histone acetylation, for example, is associated with higher transcriptional activity because this modification makes chromatin more accessible to proteins of the transcriptional apparatus (Hebbes et al., 1988). Histone deacetylases (HDACs) are a group of enzymes that catalyze the removal of acetyl groups from histones, and as such are associated with transcriptional repression.

Chapter 5 examined the anoxia-responsive expression of several HDACs in $T$. $s$. elegans. These proteins were examined in the heart, liver and white skeletal muscle of 
turtles and each tissue displayed a different pattern with respect to HDAC expression. In the muscle, a robust HDAC response was observed in response to $20 \mathrm{~h}$ of anoxia, with a uniform upregulation of all five HDACs occurring at both transcript and protein levels (FIG.5.10 and 5.11). In addition, HDAC activity in muscle increased in response to $20 \mathrm{~h}$ anoxia (FIG.5.12). Both total and acetylated histone $\mathrm{H} 3$ levels were also measured, and whereas total $\mathrm{H} 3$ levels increased, acetyl $\mathrm{H} 3$ levels were decreased in response to anoxia (FIG.5.13), further arguing for increased chromatin condensation in muscle in response to anoxia as part of global transcriptional repression. These results suggest that HDAC activation is an important mechanism of metabolic suppression in white skeletal muscle in response to anoxia.

Liver displayed a milder response, with HDAC1, -4 and -5 increasing in response to $5 \mathrm{~h}$ of anoxia (FIG.5.8 and 5.9). Levels of acetylated histone $\mathrm{H} 3$ also decreased more in response to $5 \mathrm{~h}$ than to $20 \mathrm{~h}$ of anoxia (FIG.5.13). This lesser response suggests that rather than promoting an overall state of higher chromatin condensation, liver HDACs play a role in silencing key promoter regions in response to short-term anoxia. HDAC1 is also an important mediator of cell cycle arrest via its interaction with the retinoblastoma protein $\mathrm{Rb}$ and the transcription factor $\mathrm{E} 2 \mathrm{~F}$ (Brehm et al., 1998). Therefore, upregulation of $\mathrm{HDAC} 1$ in response to $5 \mathrm{~h}$ of anoxia could be involved in cell cycle arrest in turtle hepatocytes. Indeed, the activation of HDAC1 in this tissue correlates with activation of FoxOs and p27kip, as observed in Chapter 4.

The heart showed a lesser HDAC response than either of the other tissues (FIG.5.6 and 5.7), suggesting that HDACs (with the exception of HDAC5, which was upregulated) do not play a major role in transcriptional silencing in this tissue in response 
to anoxia. This conclusion is corroborated by the data for acetylated histone $\mathrm{H} 3$ levels, which did not change significantly in the heart in response to anoxia (FIG.5.13).

The tissue specific variations in HDAC expression/activation documented in this chapter may be due to functional differences, as well as differences in the amount of metabolic suppression or the means of such suppression in these tissues. Since turtles are likely to be mostly immobile during anoxic submergences, skeletal muscle is largely unused, and a very strong metabolic suppression can occur. The liver does have important functions in providing fuel during anoxic submergence, but metabolic rate is still known to significantly decrease, creating a need for mechanisms involved in metabolic suppression. By contrast, the heart remains active throughout the anoxic episode, although beating at a lower rate, and transcriptional silencing in this tissue might not be as prominent as it is in liver or muscle. Alternately, it is possible transcriptional silencing in heart occurs by mechanisms other than chromatin condensation.

\section{ANOXIA TOLERANCE AND METABOLIC CHANGES}

This section of my thesis examined the effect of anoxia on an important metabolic transcription factor. Since any fuel sources that require complete catabolism via oxidative phosphorylation to generate ATP become obsolete during anoxia, and glycolysis becomes the primary energy pathway, it was of interest to investigate the effects of anoxia on transcriptional responses involved in the regulation of carbohydrate metabolism. The transcription factor that I focused on was ChREBP, a glucose responsive transcription factor that has been found to be an important mediator of carbohydrate metabolism in the cell (reviewed by Towle, 2005). This transcription factor 
is activated in response to high glucose levels and mediates the transcription of genes involved in glycolysis (reviewed by Towle, 2005).

Chapter 6 examined the activation of ChREBP by assessing transcript and protein expression under aerobic and anoxic conditions, as well as nuclear localization and DNAbinding activity. Overall, a strong activation of this transcription factor was found in the liver during the hypoxic transition stage ( $5 \mathrm{~h}$ of anoxic exposure), but not in response to $20 \mathrm{~h}$ of anoxia (FIG.6.5, 6.6 and 6.7).

Increased expression of the glycolytic target gene of ChREBP, liver pyruvate kinase (LPK), was also observed in response to $5 \mathrm{~h}$ of anoxia (FIG.6.8). These results suggest that ChREBP might be involved in enhancing glycolysis during the hypoxic transition stage of anoxia, when oxygen becomes limited, but metabolic rate is not yet fully depressed. Levels and activation of ChREBP returned to normoxic values by $20 \mathrm{~h}$ of anoxia, suggesting that at this point, the metabolic demands of the cell are sufficiently lowered, so that an elevated glycolytic rate is no longer necessary. Overall, these results suggest that ChREBP is an important mediator of the normoxic-hypoxic transition in turtle liver.

\section{CROSS TALK AND THE "EVERYTHING IS CONNECTED" HYPOTHESIS}

As seen throughout this discussion, many of the pathways/processes examined in my thesis are involved in more than one aspect of anoxia tolerance and "cross-talk" does seem to occur between pathways (summarized in FIG.7.1). In this section I will discuss some of the links between the various pathways examined to establish an overall picture of the anoxia responses exhibited by $T$. s. elegans. 
The UPR and NF- $\mathrm{KB}$ were the primary stress-responsive pathways examined in this thesis. While the main function of the unfolded protein response is to reduce the levels of unfolded proteins in the ER, it has also been shown to play an important role in the activation of cellular antioxidant defenses. As mentioned in Chapter 2, activated PERK can phosphorylate the transcription factor Nrf2, resulting in its activation (Cullinan et al, 2003; Cullinan and Diehl, 2004). Nrf2 is a major activator of antioxidant gene transcription (Cullinan et al, 2003) and UPR-mediated activation of this transcription factor can result in the activation of multiple cellular defenses. Therefore, in addition to the NF- $\mathrm{KB}$ and FoxO mediated induction of antioxidant defenses, a UPRmediated activation of these defenses could also potentially occur in the turtle in response to anoxia.

In addition, it has been shown that PERK-mediated phosphorylation of eIF $2 \alpha$, leading to translational inhibition, results in the clearance of short-lived proteins from the

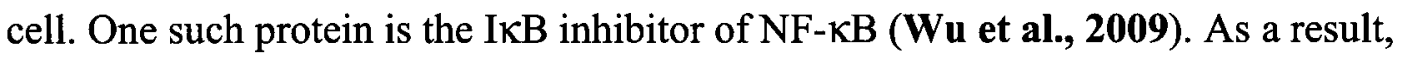
activation of NF- $\kappa B$ can occur in a UPR-related manner (Pahl andBaeuerle, 1995). Indeed, both the UPR and NF- $\kappa \mathrm{B}$ were shown to be activated in turtle liver in response to anoxia (Chapters 3 and 4), and therefore activation of the UPR could also be a factor in the NF- $\kappa B$ activation observed.

Another result of UPR-mediated translational inhibition is the clearance of D-type cyclins from the cell, leading to cell cycle arrest at G1 (Brewer et al., 1999; Brewer and Diehl, 2000). D-type cyclins activate cyclin-dependent kinases 4 and 6 that phosphorylate and inactivate the retinoblastoma protein Rb (Sherr, 1996; Ho and Dowdy, 2002). $R b$ represses the transcription of cyclin $E$, which is required for passage 
through the restriction point and entry into S phase (Sherr, 1996). In addition, anoxiaresponsive upregulation of GADD34 was seen in turtle liver, which can also be linked to cell cycle arrest through induction of p21 (Minami et al., 2007; Yagi et al., 2003). As mentioned above, cell-cycle arrest at G1 can also be mediated by FoxOs in a p27kipdependent manner, as well as HDAC1, which facilitates cell cycle arrest through its interaction with $\mathrm{Rb}$ and the transcription factor $\mathrm{E} 2 \mathrm{~F}$. All of these proteins were induced in turtle liver in response to $5 \mathrm{~h}$ of anoxia and all could contribute to anoxia-responsive cell cycle suppression in this tissue.

Finally, some overlap can also occur at the level of metabolism. In addition to their contributions to the cellular processes mentioned above, FoxOs can also contribute to the regulation of metabolism through their effects in the liver, muscle, adipose tissue and pancreas (Barthel et al., 2005). Specifically, FoxOs have been shown to regulate many metabolic genes, such as glucose-6-phosphatase (G6Pase), the G6P transporter, phosphoenolcarboxykinase (PEPCK) and PGC-1 $\alpha$ (Barthel et al., 2005). Both G6Pase and PEPCK are involved in gluconeogenesis and glycogenolysis in liver and could play a key role in anoxia survival, by supplementing the glucose provided from glycogenolysis with sugar made by catabolizing selected amino acids or by recycling some of the accumulated lactate. Therefore, induction of these enzymes in response to anoxia could have an important physiological role. Although levels of these enzymes were not examined in this thesis, they could potentially be upregulated in a FoxO-dependent manner in response to anoxia in the liver, and contribute to the capacity for glucose release by the liver into the bloodstream. 


\section{OUTLOOK: NEW QUESTIONS, NEW PATHWAYS AND NEW DIRECTIONS}

While my thesis has provided new information on many processes involved in anoxia survival at the molecular level, some new questions have emerged.

The stress response

The data presented in Chapter 2 showed that activation of the PERK branch of the UPR occurred in response to anoxia, but also hinted at possible activation of the other branches, controlled by IRE1 and ATF6. These branches should be further examined. IRE 1 is a type 1 ER-resident transmembrane protein. In response to the accumulation of unfolded proteins in the ER lumen, IRE1 undergoes trans-autophosphorylation, which activates its endonuclease activity. This activity initiates the removal of a 26 nucleotide intron from XBP1 mRNA (Yoshida et al, 2001; Calfon et al, 2002; Lee et al, 2002; Shen et al, 2001), resulting in a translational frameshift that produces a larger form of XBP1 containing a novel transcriptional activation domain in its $\mathrm{C}$-terminus. The XBP1 protein encoded by the spliced form functions as an important activator of many UPR target genes (Yoshida et al, 2001). XBP1 stimulates the expression of EDEM (Yoshida et al, 2003) and DERLIN-2 and -3 (Oda et al, 2006), both of which are components of ER-associated degradation (ERAD), a mechanism by which the cell degrades permanently unfolded or slowly folding proteins. XBP1 is also required for the full induction of many chaperone genes, such as GRP78, GRP94, p58ipk, Erdj4, ERO1-La (Lee et al, 2003) and ERO1-Lb (Sriburi et al, 2007). The anoxia-responsiveness of the IRE1-XBP1 branch of the UPR, as well as activation of the ERAD machinery would be of interest since this pathway has never been examined in turtle tissues and could potentially suggest a novel mechanism used by turtle cells to clear unfolded proteins in 
response to anoxia. Activation of this pathway could be assessed through examination of the phosphorylation status of IRE1, as well as measurement of the transcript and protein levels of spliced XBP1. Studies of the nuclear localization and DNA-binding activity of $\mathrm{XBP} 1$ would also be of value, as well as examination of the transcript and protein levels of genes involved in ERAD, such as EDEM and DERLIN2 and -3.

The third branch of the UPR is controlled by ATF6. In unstressed cells, ATF6 is localized to the ER membrane through its association with GRP78. However, ER stress and subsequent dissociation from GRP78 unmasks its Golgi localization sites, allowing it to translocate to the Golgi, where it is cleaved by two proteases (Haze et al., 1999; Li et al., 2000). The processed form of ATF6 translocates to the nucleus and activates UPR target genes, inducing expression of several chaperones (Shen et al., 2005), including GRP78, GRP94, calreticulin, and the DnaJ co-chaprone p58ipk (159), several PDIs including PDI and Erp72 (Okada et al., 2002; Lee et al., 2003) and the cargo receptor ERGIC-53 (Renna et al., 2007). In addition, ATF6 is known to induce proteins involved in ERAD, such as Herp (Kokame et al., 2001; Ma and Hendershot, 2004; Yamamoto et al., 2004). Because of its contribution to the induction of molecular chaperones, as well as ERAD-associated genes, examination of the ATF6 pathway in response to anoxia would help get a complete picture of UPR involvement in the response to anoxia in $T$. $s$. elegans. Activation of this pathway could be assessed through examination of the protein levels of active and inactive ATF6, its nuclear localization, and changes in its DNAbinding in response to anoxia. Changes in the transcript and protein levels of the ERADassociated gene Herp should also be examined. 


\section{Epigenetics and hypometabolism}

The anoxia-responsive activation of epigenetic mechanisms in turtle tissues is a particularly interesting area of study, as it suggests a previously unknown aspect of anoxia-responsive metabolic rate depression, and should be explored further. Future studies in this area should investigate the role of other epigenetic modifications in anoxic submergence in $T$. s. elegans. For example, besides histone acetylation, histone methylation plays an important role in transcription, and increased histone methylation has been associated with transcriptional repression (Biel et al., 2004). It would be of interest to study changes in the methylation levels of histones in response to anoxia in turtle tissues, as well as changes in the expression and activities of the enzymes involved in this process, the histone methyltransferases.

DNA methylation has also been associated with transcriptional repression (Nakao et al., 2001) and could represent another novel mechanism of transcriptional repression in the turtle. The role of this process in anoxia tolerance could be studied by examination of changes in the global methylation levels of DNA in various tissues, as well as changes in expression and activities of the DNA methyltransferase enzymes that are involved in catalyzing DNA methylation. Changes in the levels of methyl binding proteins, which bind to methylated $\mathrm{CpG}$ residues and recruit co-repressor proteins, thereby repressing transcription (Nakao et al., 2001), should also be examined.

\section{Cell cycle control}

As discussed above, several molecular mechanisms that were activated in turtle liver in response to $5 \mathrm{~h}$ of anoxia were indicative of cell cycle arrest. Anoxia-responsive 
cell cycle arrest in the turtle liver represents another novel mechanism of metabolic depression, largely unexplored in anoxia tolerant species, and one that should be researched further. For example, in addition to their induction of p27kip, FoxOs have been shown to contribute to cell cycle inhibition by inducing the expression of the retinoblastoma family member p130 (Kops et al., 2002a). This protein is involved in the repression of genes required for reentry of cells into the cell cycle, and therefore, its induction allows the maintenance of a quiescent state (Smith et al., 1996). Levels of this protein should be assessed under aerobic and anoxic conditions to further establish the role of FoxOs in cell cycle inhibition at G1. In addition, FoxOs can induce the transcription of cyclin G2 (Martinez-Gac et al., 2004), a cyclin that is highly expressed in quiescent cells but is significantly under-expressed as cells enter the cell cycle (Bennin et al., 2002; Horne et al., 1997). Enhanced presence of this cyclin in anoxic liver could be a further indicator of cell cycle arrest.

\section{CONCLUSIONS}

In conclusion, my thesis offers new insights into some of the transcriptional and post-translational mechanisms involved in anoxia tolerance in the turtle, $T$. s. elegans. It demonstrated the anoxia-responsive activation of multiple pathways implicated in the stress response, hypometabolism and carbohydrate metabolism in turtle tissues, and suggests that these pathways are of key importance to anoxia tolerance. In particular, the liver was shown to be the most responsive tissue with respect to most of the molecular processes analyzed, exhibiting strong activation of stress-responsive pathways and proteins (including those related to both protein unfolding and antioxidant defense), as 
well molecular induction of hypometabolism and metabolic reorganization (FIG.7.2). These results could reflect the importance of suppressing metabolic rate in the liver in response to anoxia, while still maintaining selective functions that contribute to both liver preservation and, because of the central role of liver in managing fuel metabolism, to the survival of the whole animal. 


\section{FIG.7.1}

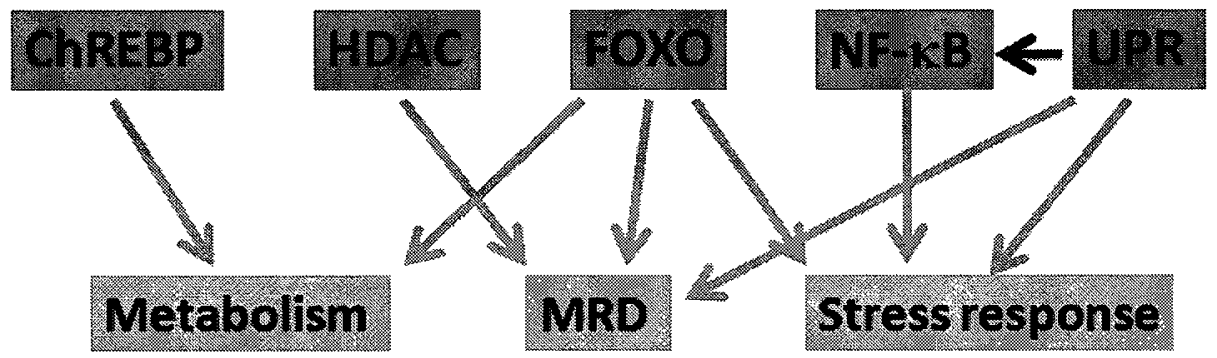

FIG.7.1. Overview of cross-talk between the different pathways examined in this thesis. 


\section{FIG.7.2}

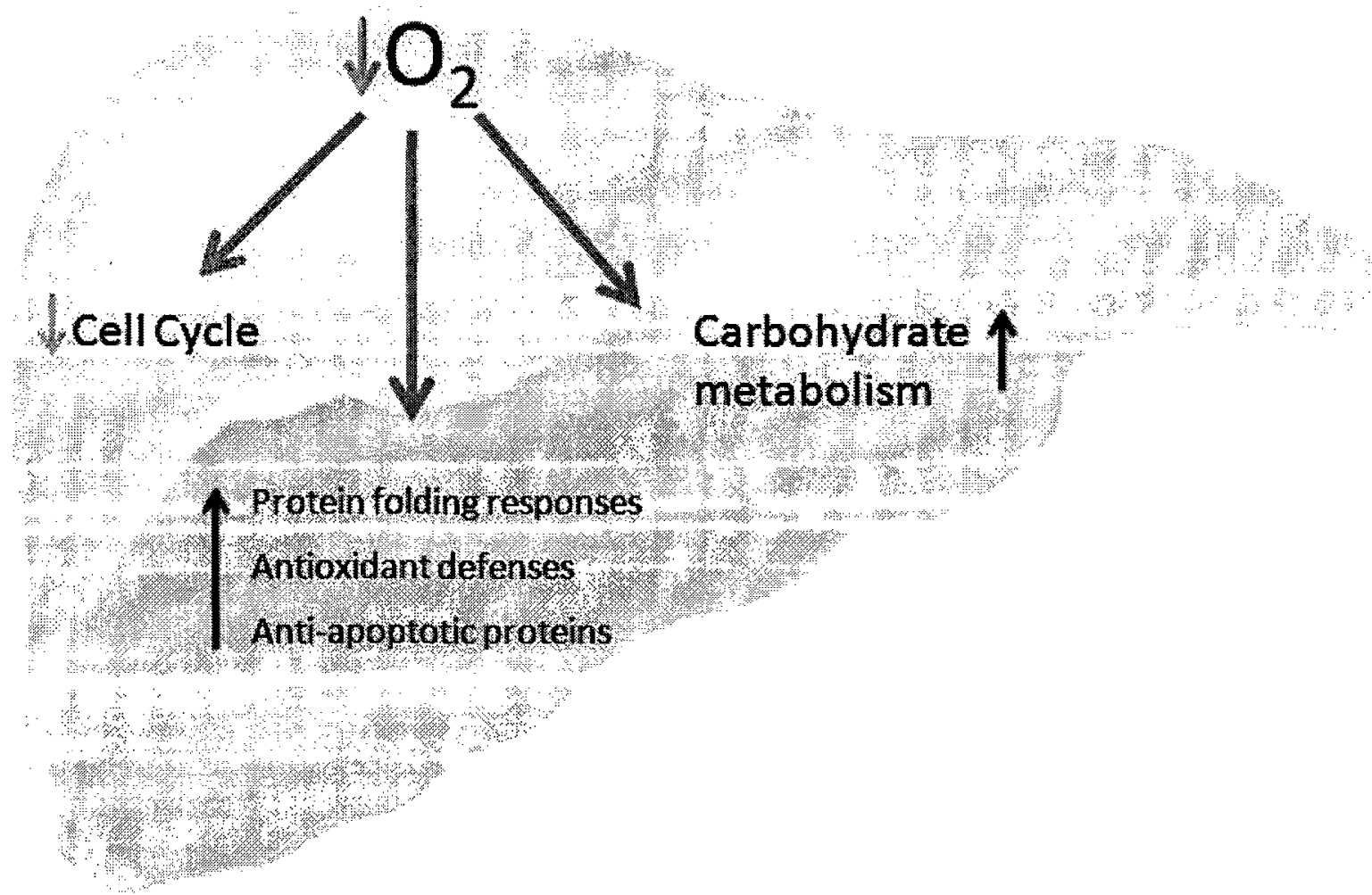

FIG.7.2. Overview of the molecular responses observed in turtle liver in response to anoxia. In response to oxygen deprivation, the liver activates multiple adaptive mechanisms. These include metabolic rate depression (at least in partially as a result of cell cycle supression), activation of various stress-responsive pathways to protect and preserve hepatocytes over prolonged periods of anaerobiosis, and activation of carbohydrate metabolism to meet energy demands. 
APPENDICES 


\section{Appendix A: List of Publications}

\section{Published/In press:}

Krivoruchko A, Storey KB. Regulation of the heat shock response under anoxia in the turtle, Trachemys scripta elegans. J Comp Physiol B. 2010;180(3):403-14.

Krivoruchko A, Storey KB. Molecular mechanisms of turtle anoxia tolerance: A role for NF-kappaB. Gene. 2010;450(1-2):63-9.

Krivoruchko A, Storey KB. Activation of antioxidant defenses in response to freezing in freeze-tolerant painted turtle hatchlings. Biochim Biophys Acta. $2010 ; 1800(7): 662-668$.

Krivoruchko A, Storey KB. Epigenetics in anoxia tolerance: a role for histone deacetylases. Mol Cell Biochem. In press. Epub: May 2010.

Krivoruchko A, Storey KB. Forever young: mechanisms of natural anoxia tolerance and potential links to longevity. Oxid Med Cell Longev. Review. In press.

\section{In preparation:}

Krivoruchko A, Storey KB. Coping with stress: activation of the unfolded protein response in response to anoxia in the turtle, Trachemys scripta elegans. In preparation.

Krivoruchko A, Storey KB. Activation of the FoxO transcription factors in an anoxia-tolerant vertebrate. In preparation.

Krivoruchko A, Storey KB. Activation of the Carbohydrate Response Element Binding Protein in response to anoxia in the turtle Trachemys scripta elegans. In preparation.

Krivoruchko A, Storey KB. Activation of the heat shock response during freezing in freeze-tolerant hatchling turtles. In preparation. 


\section{Appendix B: Communications at scientific meetings}

\section{Poster presentations:}

Krivoruchko, A. and Storey, K.B. Expression of heat shock proteins in an anoxia-tolerant turtle, Trachemys scripta elegans. College of Natural Science Research Day. Ottawa, ON. April 6, 2006.

Krivoruchko, A. and Storey, K.B. Expression of heat shock proteins in an anoxia-tolerant turtle, Trachemys scripta elegans. Ottawa Carleton Chemistry Institute Poster Day. Ottawa, ON, May 19, 2006.

Krivoruchko, A. and Storey, K.B. Expression of heat shock proteins in an anoxia-tolerant turtle, Trachemys scripta elegans. 9th Annual Chemistry \& Biochemistry Graduate Research Conference, Concordia University, Montreal, PQ, November 24-25, 2006.

Krivoruchko, A. and Storey, K.B. Differential expression of peroxiredoxins in an anoxiatolerant turtle. 5th meeting, Canadian Oxidative Stress Consortium, Montreal, PQ, May 3-6 2007.

Krivoruchko, A. and Storey, K.B. Differential expression of peroxiredoxins in an anoxiatolerant turtle. 10th Annual Chemistry \& Biochemistry Graduate Research Conference, Concordia University, Montreal, PQ, November 23-24, 2007.

Krivoruchko, A. and Storey, K.B. Differential expression of peroxiredoxins in an anoxiatolerant turtle. $5^{\text {th }}$ annual Ottawa-Carleton Institute of Biology Symposium. Ottawa, ON, April 28-29, 2008.

Krivoruchko, A. and Storey, K.B. Differential expression of peroxiredoxins in an anoxiatolerant turtle. Annual meeting of the American Society of Ichthyologists and Herpetologists. Montreal, PQ, July 23-28, 2008.

Krivoruchko, A. and Storey, K.B. Activation of ChREBP in response to anoxia in the anoxia tolerant turtle, Trachemys scripta elegans. 11th Annual Chemistry \& Biochemistry Graduate Research Conference, Concordia University, Montreal, PQ, November 21-22, 2008. 
Krivoruchko, A. and Storey, K.B. Activation of ChREBP in response to anoxia in the anoxia tolerant turtle, Trachemys scripta elegans. $6^{\text {th }}$ annual Ottawa-Carleton Institute of Biology Symposium. Ottawa, ON, April 30-May 1, 2009.

Krivoruchko, A. and Storey, K.B. Differential expression of peroxiredoxins in an anoxiatolerant turtle. 34th annual APICS/CIC Student Chemistry Conference, Antigonish, NS, May 14th -16th, 2009.

Krivoruchko, A. and Storey, K.B. Activation of ChREBP in response to anoxia in the anoxia tolerant turtle, Trachemys scripta elegans. $48^{\text {th }}$ annual meeting of the Canadian Society of Zoologists. University of Toronto Scarborough. Toronto, ON. May 12-16, 2009.

\section{Invited Symposium Presentations:}

Krivoruchko, A. Tales from the turtle pond: molecular mechanisms of anoxia tolerance in overwintering $t$ urtles. The $88^{\text {th }}$ Annual meeting of the American Society of Ichthyologists and Herpetologists. Montreal, PQ, July 23-28, 2008.

\section{Other Oral Presentations:}

Krivoruchko, A. Molecular mechanisms of anoxia tolerance in turtles: a role for NF-kB. 11th Annual Chemistry \& Biochemistry Graduate Research Conference, Concordia University, Montreal, PQ, November 21-22, 2008. (Winner for best Molecular Biology presentation)

Krivoruchko, A. Molecular mechanisms of anoxia tolerance in turtles: a role for NF-kB. $48^{\text {th }}$ annual meeting of the Canadian Society of Zoologists. University of Toronto Scarborough. Toronto, ON. May 12-16, 2009. 


\section{Appendix C: List of GenBank submissions}

Krivoruchko A, Storey KB. Trachemys scripta elegans heat shock factor 1 (HSF1) mRNA, partial cds. EU185740

Krivoruchko A, Storey KB. Trachemys scripta elegans X-linked inhibitor of apoptosis mRNA, partial cds. FJ834445

Krivoruchko A, Storey KB. Trachemys scripta elegans baculoviral IAP repeat-containing 2 mRNA, partial cds. FJ834444

Krivoruchko A, Storey KB. Trachemys scripta elegans copper, zinc superoxide dismutase mRNA, partial cds. FJ834443

Krivoruchko A, Storey KB. Trachemys scripta elegans manganese superoxide dismutase mRNA, partial cds. FJ834442

Krivoruchko A, Storey KB. Trachemys scripta elegans ferritin heavy chain mRNA, partial cds. FJ834441

Krivoruchko A, Storey KB. Trachemys scripta elegans nuclear factor of kappa light polypeptide gene enhancer in B-cells 1 mRNA, partial cds. FJ834440

Krivoruchko A, Storey KB. Trachemys scripta elegans NF-kappaB p65 subunit protein mRNA, partial cds. FJ834439

Krivoruchko A, Storey KB. Trachemys scripta elegans alpha-tubulin mRNA, partial cds. FJ834438

Krivoruchko A, Xie L, Storey KB. Trachemys scripta elegans Bcl-xL mRNA, partial cds. FJ884694

Krivoruchko A, Storey KB. Trachemys scripta elegans B-cell lymphoma protein 2 (Bcl2) mRNA, partial cds. FJ884693

Krivoruchko A, Storey KB. Chrysemys picta marginata nuclear factor (erythroid-derived 2)-like 2 (Nrf2) mRNA, partial cds. GU170376 


\title{
Appendix D: Extra amino acid alignments
}

\author{
1. ATF3
}

T. s. elegans

G. gallus

H. sapiens

$X$. Iaevis

D. rerio

T. s. elegans

G. gallus

H. sapiens

$X$. laevis

D. rerio

T. S. elegans

G. gallus

H. sapiens

$X$. Iaevis

D. rerio

T. s. elegans

G. gallus

$H$. sapiens

$X$. laevis

D. rerio

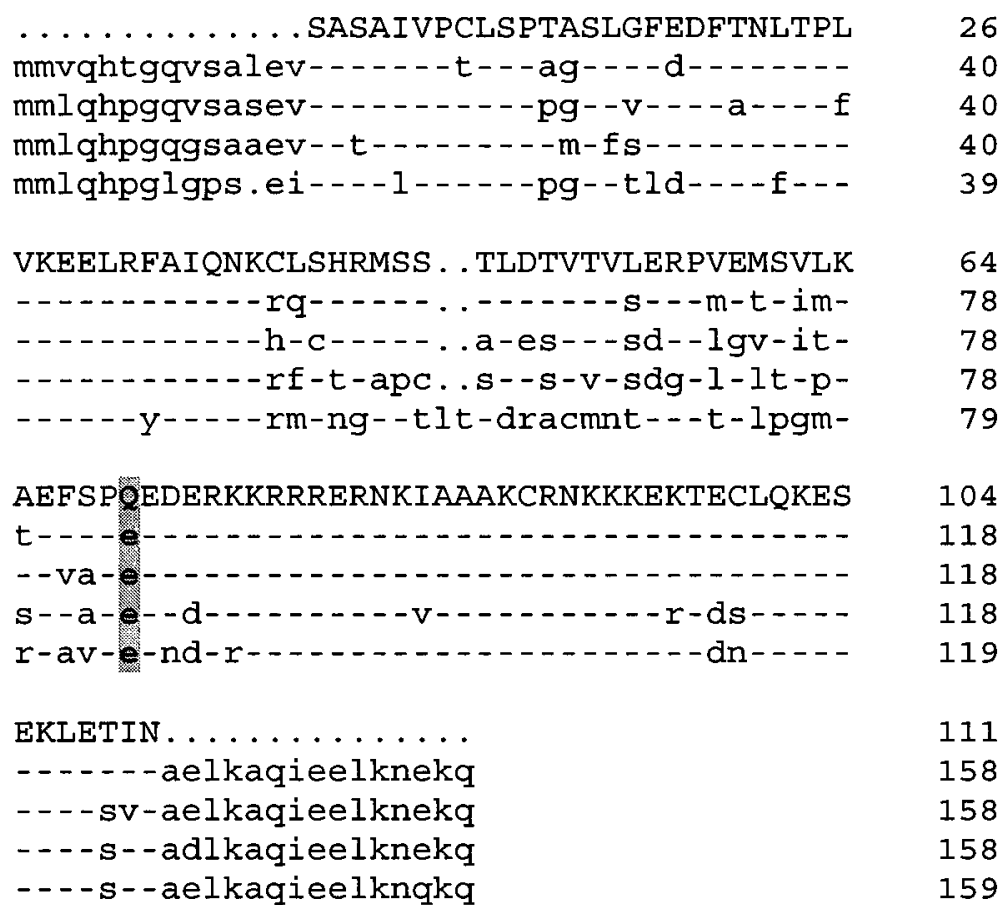

FIG. 1. The partial amino acid sequence of ATF3 from T. s. elegans compared with ATF3 sequences from human (Homo sapiens), chicken (Gallus gallus), African clawed frog (Xenopus laevis), and zebrafish (Danio rerio). Accession numbers are XP_419429 for chicken, NP 001665 for human, NP 001087487 for frog and NP 957258 for zebrafish. Dashes replace amino acid residues identical with $T$. $s$. elegans while spacer dots indicate residues that are missing in the sequence. Turtle-specific amino acid substitution is bolded and shaded. 


\section{GRP78}

T. s. elegans

G. gallus

H. sapiens

$X$. laevis

D. rerio

T. s. elegans

G. gallus

H. sapiens

$X$. laevis

$D$. rerio

T. s. elegans

........ ENTVFDAKRLIGRTWNDPSVQQDI KYLPFK

aaknqltsnp-_....................... 116

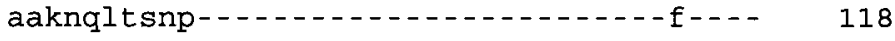

aaknqltsnp- - . -

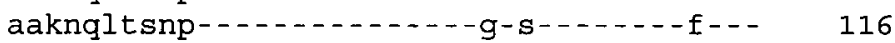

G. gallus

H. sapiens

$X$. laevis

D. rexio

VVEKKTKPYIQVDVGGGQTKTFAPEEISAMVLTKMKETAE 70

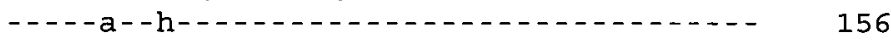

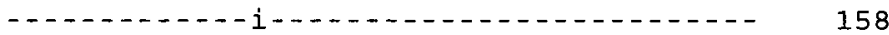

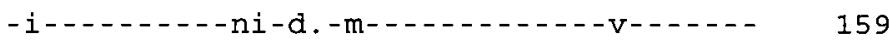

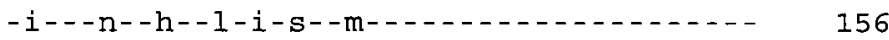

T. s. elegans

AYLGKKVTHAVVTVPAYFNDAQRQATKDAGTIAGLNVMRI 110

-

-

- . -

- - - -

G. gallus

INEPTAAAIAYGLDKREGEKNILVFDLGGGTFDVSLLTID 150

IN-10.0.

- -

. sapiens

-1-

$X$. laevis

D. rerio

T. S. elegans

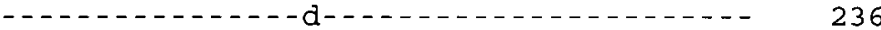

G. gallus

H. sapiens

$X$. Iaevis

NGVFEVVATNGDTHLGGEDFDQRVMEHF I KLYKKKTGKDV 190

_...

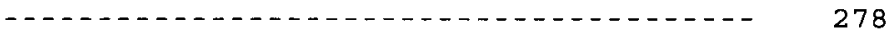

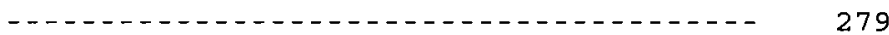

D. rerio

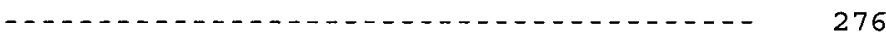

T. S. elegans

RKDNRSVQKLRREVEKAKRALSSQHQARIEIESFFEGEDF 230

G. gallus

H. sapiens

$X$. laevis

D. rerio

T. S. elegans

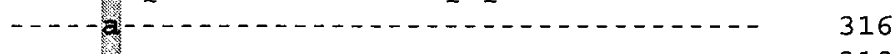

- - -

- - -

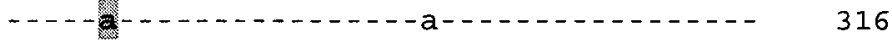

G. gallus

H. sapiens

$X$. laevis

D. rerio

SETLTRAKFEELNMDLFRSTMKPVQKVLEDSDLKKSDIDE 270

-

-

-

- 356

T. s. elegans

IVLVGGSTR. . . . . . .

279

------ipkiqqlvkeff

396

G. gallus

H. sapiens

$X$. laevis

D. rerio

--ipkiqqlvkeff

398

-...--ipkiqqlvkeff 399

-...---ipkiqqlvkeff 396

FIG. 2. The partial amino acid sequence of GRP78 from $T$. s. elegans compared with GRP78 sequences from human (Homo sapiens), chicken (Gallus gallus), African clawed frog (Xenopus laevis), and zebrafish (Danio rerio). Accession numbers are NP_990822 for chicken, NP_005338 for human, NP_001080064 for frog and AAH63946 for zebrafish. Dashes replace amino acid residues identical with $T$. s. elegans while spacer dots indicate residues that are missing in the sequence. Turtle-specific amino acid substitution is bolded and shaded. 


\section{GRP94}

T. s. elegans

G. gallus

H. sapiens

$X$. Iaevis

D. rerio

T. s. elegans

G. gallus

H. sapiens

$X$. Iaevis

D. rerio

T. s. elegans

G. gallus

$H$. sapiens

$X$. laevis

D. rerio

T. s. elegans

G. gallus

$H$. sapiens

$X$. laevis

D. rerio

T. S. elegans

G. gallus

H. sapiens

$X$. laevis

D. rerio

T. s. elegans

G. gallus

H. sapiens

$X$. laevis

D. rerio

T. s. elegans

G. gallus

$H$. sapiens

$X$. laevis

D. rerio
KSFSKEHDD PMAYIHFTAEGEVTFKSILFI PTXAPRGLFD 92

-

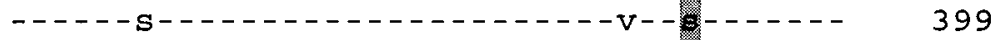

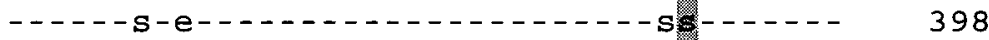

-t--rdt-e-lsh--------------v-as------ 399

EYGSKKSDYIKLYVRRVFITDDFHDMMPKYLNFVKGVVDS 132

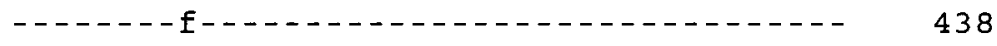

- -

$-------\mathrm{f}---\mathrm{f}---------------------\quad 438$

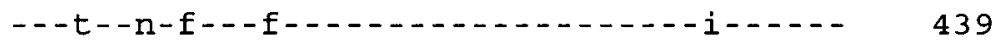

DDLPLNVSRETLQQHKLLKVIRKKLVRKTLDMIKKIAEEK 172

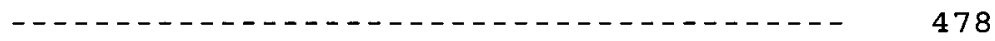

- - - - - - - - - - - -

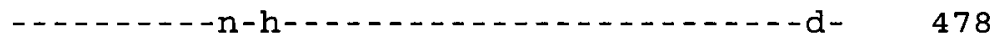

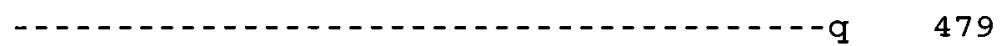

YNDTFWKEFGTNIKLGVIEDHSNRTRLAKLLRFQSSHHES 212

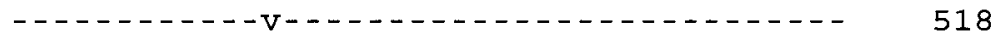

- - -

- t-k- - - n

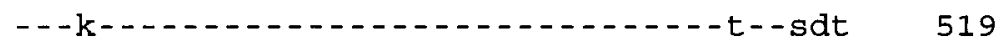

NTTSLDQYVERMKEKQDKIYFMAGSIYRKEAESSPFVERLL 252

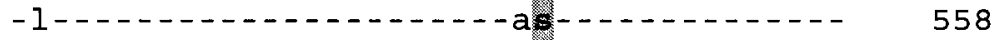

di-_..-

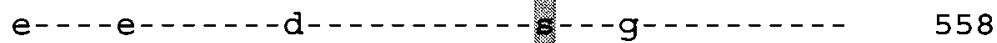

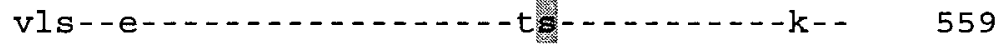

KKGYEVIYLTEPVDEYCIQALPEFDGKRFQNVAKEGVKFD 292

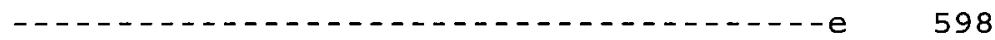

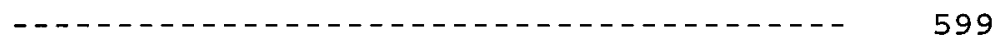

- - - - vf-i--- - - - - - - - - - - - - - lq- 598

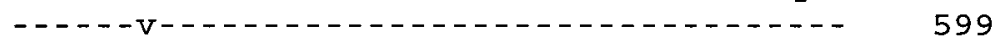

ESDKSKESREALEKEFEPLLTWMKD . . . . . . . . 317

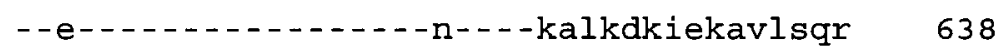

--e-t------v---.---n----kalkdkiekavvsqr 639

-ne----v-------y-------ekalkdqiekavvsqr 638

----a--k---------t-----kalkeqiekavlsqr 639

FIG. 3. The partial amino acid sequence of GRP94 from T. s. elegans compared with GRP94 sequences from human (Homo sapiens), chicken (Gallus gallus), African clawed frog (Xenopus laevis), and zebrafish (Danio rerio). Accession numbers are NP_989620 for chicken, NP_003290 for human, NP_001084280 for frog and NP_937853 for zebrafish. Dashes replace amino acid residues identical with $T$. s. elegans while spacer dots indicate residues that are missing in the sequence. Turtle-specific amino acid substitutions are bolded and shaded. 


\section{4. $\mathrm{p50}$}

T. s. elegans

G. gallus

H. sapiens

$X$. laevis

T. s. elegans

G. gallus

H. sapiens

$X$. laevis

T. S. elegans

G. gallus

H. sapiens

$X$. laevis

T. S. elegans

G. gallus

H. sapiens

$X$. laevis

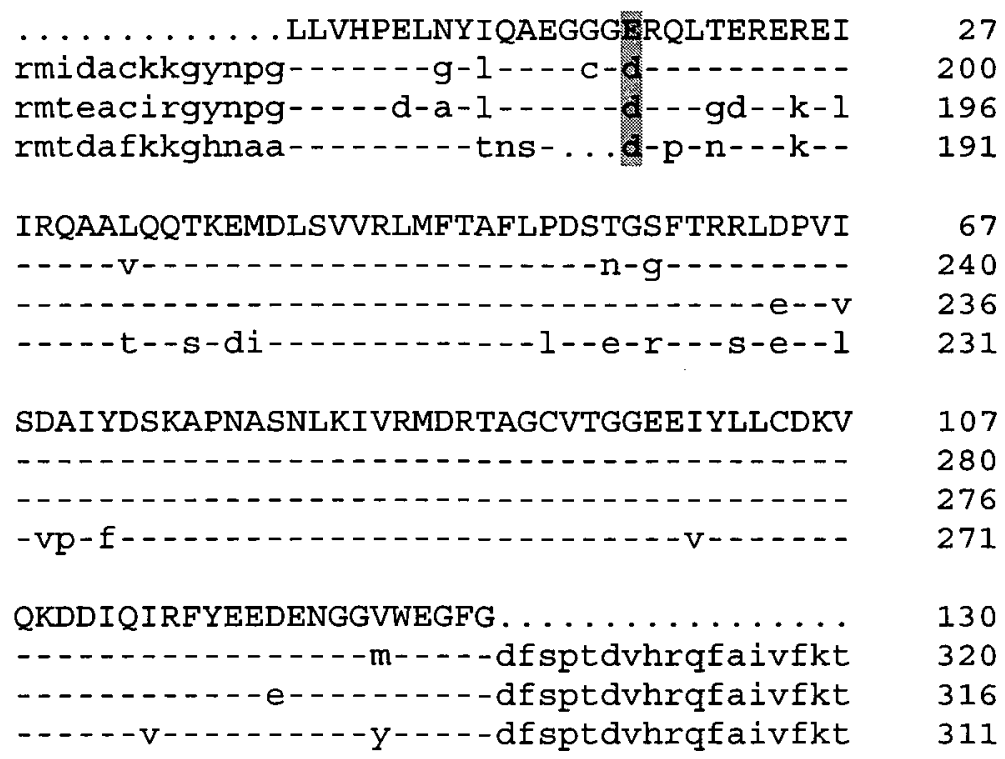

27
200

196

191

FIG. 4. The partial amino acid sequence of p50 from $T$. s. elegans compared with p50 sequences from human (Homo sapiens), chicken (Gallus gallus) and African clawed frog (Xenopus laevis). Accession numbers are ACO87295 for turtle, NP_990465 for chicken, NP_003989 for human and NP_001087808for frog. Dashes replace amino acid residues identical with $T$. s. elegans while spacer dots indicate residues that are missing in the sequence. Turtle-specific amino acid substitution is bolded and shaded. 


\section{5. $\mathrm{p} 65$}

T. S. elegans

.VLGIQCVKKRELDEAVAQRIRTNNNPFNVPLDNQKGDY . 38

G. gallus

H. sapiens

$X$. laevis

D. rerio

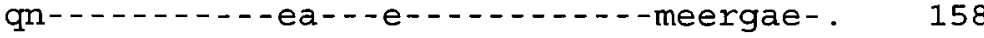

qn--...--d-eq-is---q-----q--iee-r---. 152

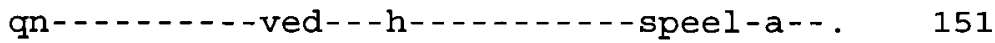

qn-1-.---kdvg---sc-lq-q---ki-dakiweeef 151

T. S. elegans

DLNAVRLCFQVWVQDPV. GTGHLVHLPLVVSQPI YDNRAP 77

G. gallus

H. sapiens

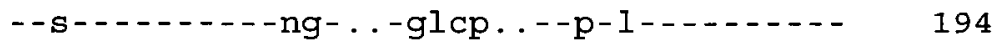

$X$. laevis

D. rerio

-...-.---t-r--s.-rplr..--p-l-h--f-..- 189

---t-c----fip-qaa-rmlp..--f---_-..-.- 189

--.---r-sitlss.-dlfp..-ep-.-.-...- 188

T. S. elegans

NTAELKICRVNR. . . . . . . . . . . . . 89

G. gallus

H. sapiens

$X$. laevis

D. rerio

s----r-lpgd-nsgscqggdeifllcdkvqkedievrfw 234

-......-nsgsclggdeifllcdkvqkedievyft 229

- - - - - knsgsclggdeifllcdkvqkedievifg 229

- . - nsgscrggdeifllcdkvqkedievrff 228

FIG. 5. The partial amino acid sequence of $\mathrm{p} 65$ from $T$. $s$. elegans compared with $\mathrm{p} 65$ sequences from human (Homo sapiens), chicken (Gallus gallus), African clawed frog (Xenopus laevis) and zebrafish (Danio rerio). Accession numbers are ACO87294 for turtle, NP_990460 for chicken, NP_068810 for human, NP_001081048 for frog and NP_001001839 for zebrafish. Dashes replace amino acid residues identical with $T$. $s$. elegans while spacer dots indicate residues that are missing in the sequence. 


\section{Ferritin heavy chain}

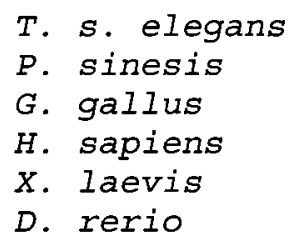

P. sinesis

G. gallus

H. sapiens

$X$. laevis

D. rerio

T. S. elegans

P. sinesis

G. gallus

H. sapiens

$X$. laevis

D. rerio

T. S. elegans

P. sinesis

G. gallus

H. sapiens

$X$. laevis

D. rerio

T. S. elegans

P. sinesis

G. gallus

H. sapiens

$X$. laevis

D. rerio

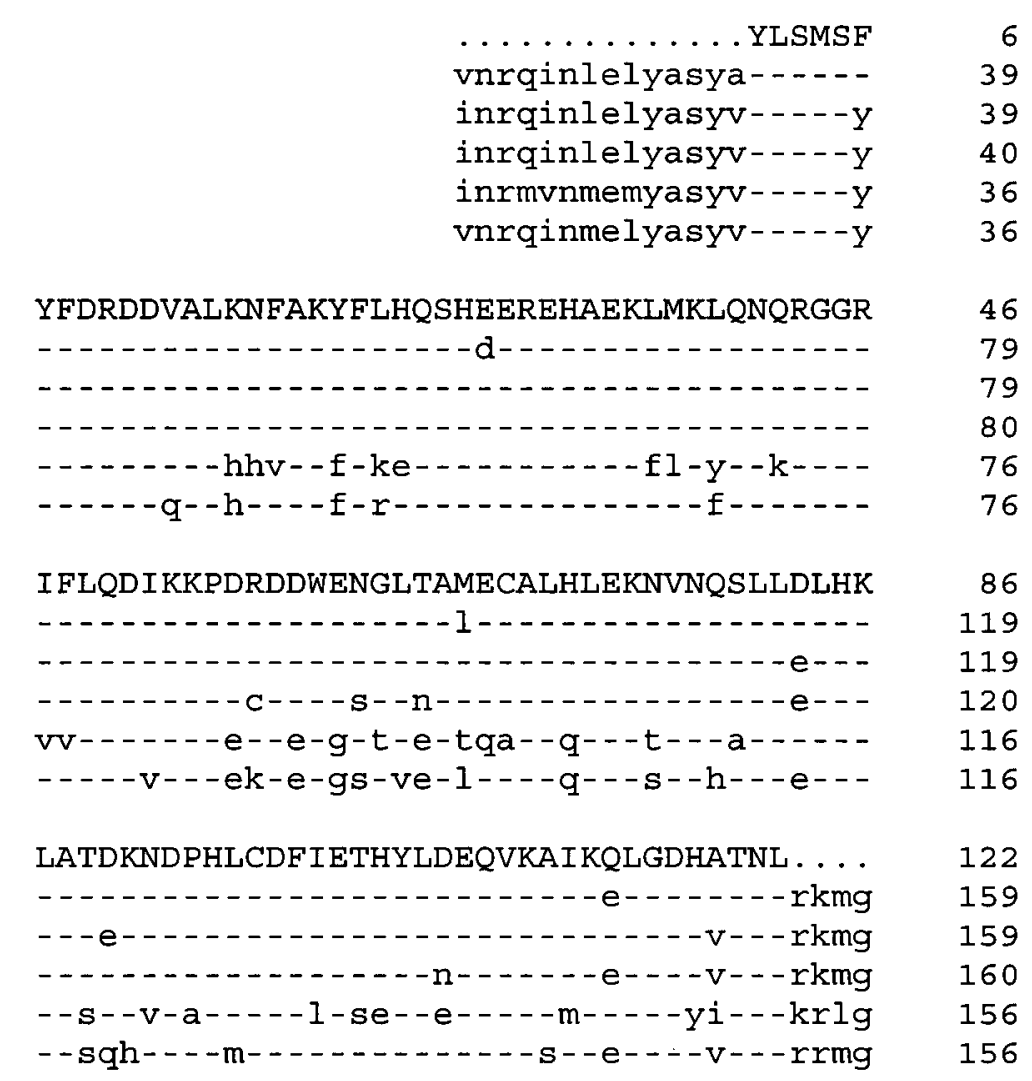

FIG. 6. The partial amino acid sequence of ferritin heavy chain from T. s. elegans compared with ferritin heavy chain sequences from soft-shell turtle (Pelodiscus sinensis), human (Homo sapiens), chicken (Gallus gallus), African clawed frog (Xenopus laevis) and zebrafish (Danio rerio). Accession numbers are ACO87296 for red-eared slider turtle, AAY64004 for soft-shell turtle, NP_990417 for chicken, NP_002023 for human, NP_ 001084057 for frog and NP_571660 for zebrafish. Dashes replace amino acid residues identical with $T$. $s$. elegans while spacer dots indicate residues that are missing in the sequence. 


\section{Mn SOD}

T. s. elegans

G. gallus

H. sapiens

$X$. laevis

D. rerio

T. s. elegans

G. gallus

H. sapiens

$X$. laevis

D. rerio

T. s. elegans

G. gallus

$H$. sapiens

$X$. laevis

D. rerio

T. s. elegans

G. gallus

H. sapiens

$X$. laevis

D. rerio

T. S. elegans

G. gallus

$H$. sapiens

$X$. laevis

D. rerio

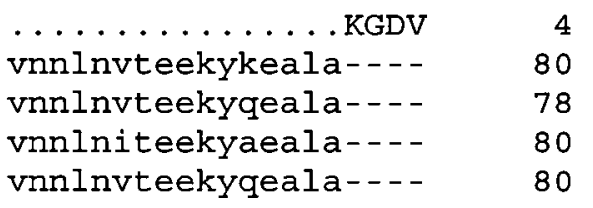

TAQVSLQPALKFNGGGHINHTIFWTNLSPNGGGEPQGELM 44

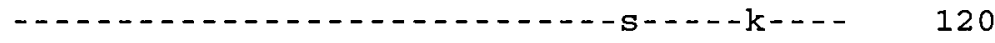

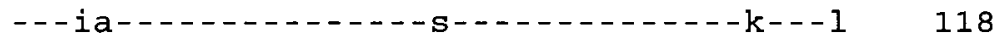

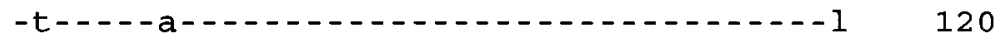

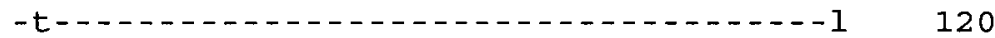

EAIKRDFGSFGNFKEKLTAVSVGVQGSGWGWLGFNQDQGR 84

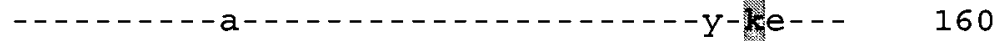

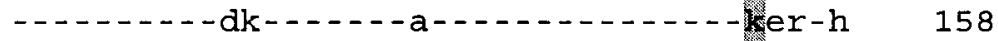

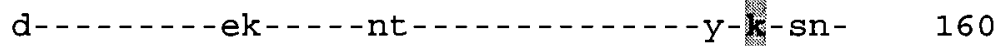

--------qkm---is-at-a---------elkes-- 160

LQVIACYNQDPLQGTTGLI PLLGIDVWEHAYYLQYKNVRP 124

- $\mathrm{i}$ Q

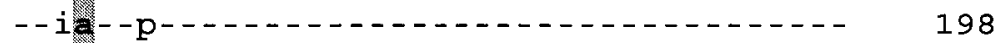

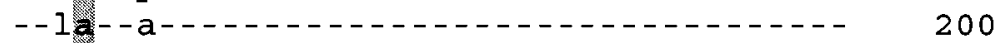

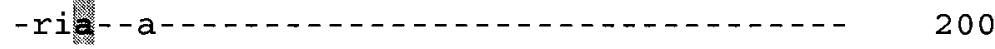

DYLKAIWN............. 132

-..----vinwenvsqryescrk 224

-------vinwenvterymackk $\quad 222$

-.-.---vinwenvteryqaskk 224

--v--.--vvnwenvserfqaakk 224

FIG. 7. The partial amino acid sequence of Mn SOD from $T$. s. elegans compared with MnSOD sequences from human (Homo sapiens), chicken (Gallus gallus), African clawed frog (Xenopus laevis) and zebrafish (Danio rerio). Accession numbers are ACO87297 for turtle, NP_989542 for chicken, NP_001019636 for human, NP_001083968 for frog and NP_956270 for zebrafish. Dashes replace amino acid residues identical with $T$. s. elegans while spacer dots indicate residues that are missing in the sequence. Turtle-specific amino acid substitutions are bolded and shaded. 


\section{Cu/Zn SOD}

T. s. elegans

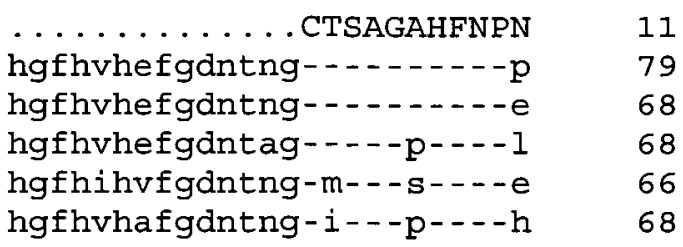

C. carreta

G. gallus

H. sapiens

$X$. laevis

D. rerio

T. s. elegans

GKNHGGPQDKERHVGDLGNVIANKDGVAEVSIKDSLISLT 51

C. carreta

G. gallus

H. sapiens

$X$. Iaevis

D. rerio

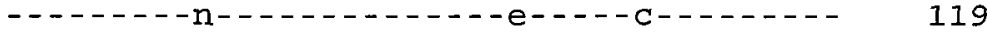

--q----k-ad---------t-.-g-----e-e--v---- 107

srk-.--k-e-.-.-.--t-d--.--d---e--v--s 108

n----a-g-td-.-.-.--t-.eg---qfk-t-----k 105

d-t----t-sv-------t-das---kie-e-amlt-s 108

T. s. elegans

GPLSIIGRTMVVHEKEDDLGKGNNN. ......... 75

C. carreta

G. gallus

H. sapiens

$X$. laevis

D. rerio

-sq-- - - - - - - - - - g-deslktgnagsrlacg 159

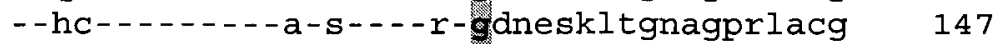

-dhc---1-1----a-----g-eestktgnagsrlacg 148

--n-----a----a-----g-deslktgnaggrlacg 145

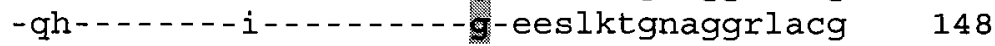

FIG. 8. The partial amino acid sequence of $\mathrm{Cu} / \mathrm{Zn}$ SOD from $T$. s. elegans compared with $\mathrm{Cu} / \mathrm{Zn}$ SOD sequences from loggerhead turtle (Carreta carreta), human (Homo sapiens), chicken (Gallus gallus), African clawed frog (Xenopus laevis) and zebrafish (Danio rerio). Accession numbers are ACO87298 for red-eared slider turtle, AAB25456 for loggerhead turtle, NP_990395 for chicken, NP_000445 for human, NP_001080933 for frog and NP_571369 for zebrafish. Dashes replace amino acid residues identical with T. $s$. elegans while spacer dots indicate residues that are missing in the sequence. Redeared slider-specific amino acid substitution is bolded and shaded. 


\section{Bcl-2}

T. s. elegans

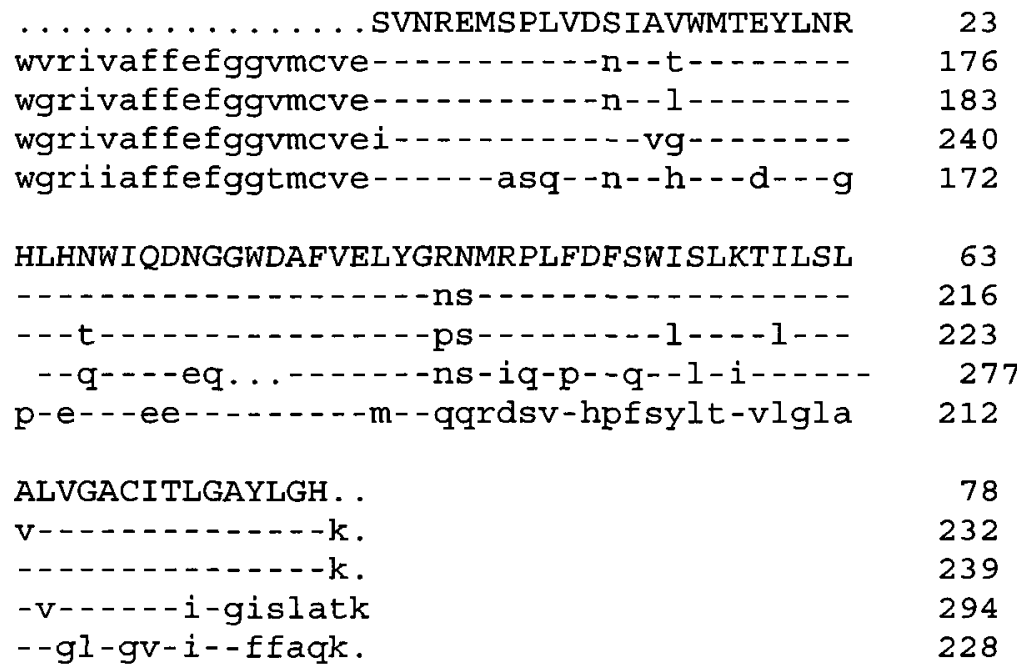

FIG. 9. The partial amino acid sequence of Bcl-2 from $T$. s. elegans compared with Bcl-2 sequences from human (Homo sapiens), chicken (Gallus gallus), African clawed frog (Xenopus laevis) and zebrafish (Danio rerio). Accession numbers are ACQ42285 for turtle, NP_990670 for chicken, NP_000624 for human, NP_001139565 for frog and NP_001025424 for zebrafish. Dashes replace amino acid residues identical with $T$. $s$. elegans while spacer dots indicate residues that are missing in the sequence. 


\section{0. p27kip1}

T. s. elegans

G. gallus

H. sapiens

D. rerio

T. s. elegans

G. gallus

H. sapiens

D. rerio

T. S. elegans

G. gallus

H. sapiens

D. rerio

T. S. elegans

G. gallus

H. sapiens

D. rerio

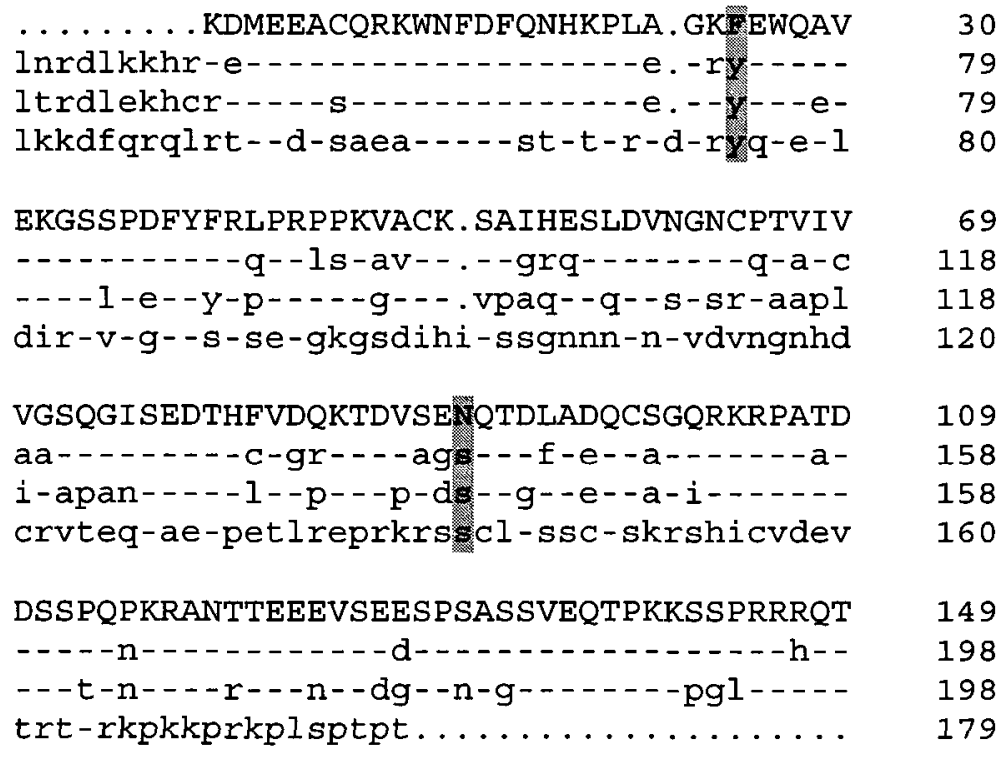

FIG. 10. The partial amino acid sequence of $\mathrm{p} 27 \mathrm{kip} 1$ from $T$. s. elegans compared with p27kip1 sequences from human (Homo sapiens), chicken (Gallus gallus) and zebrafish (Danio rerio). Accession numbers are NP_989587 for chicken, NP_004055 for human and NP_997957 for zebrafish. Dashes replace amino acid residues identical with $T . s$. elegans while spacer dots indicate residues that are missing in the sequence. Turtlespecific amino acid substitutions are bolded and shaded. 


\section{Catalase}

T. S. elegans

....FEVTHDITKYCKAKVFEHIGKRTPIAIRFSTVAGES 36

G. gallus

$H$. sapiens

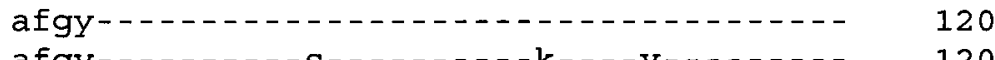

$X$. laevis

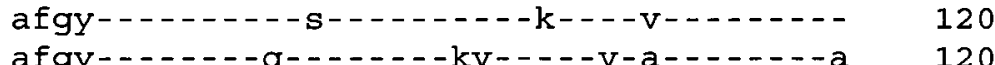

D. rerio

afgY-------r-s------v--t--.-v-------a 120

T. s. elegans

GSADTVRDPRGFAMKFYTEEGIWDLVGNNTPIFFIRDAML 76

G. gallus

H. sapiens

-

$X$. laevis

D. rerio

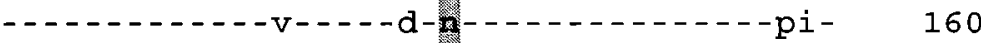

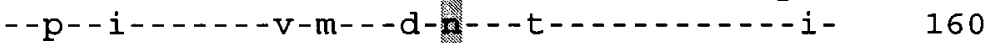

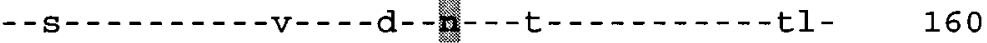

T. s. elegans

FPSFIHSQKRNPQTHLKDPDMVWDFWSLRPESLHQVSFLF 116

G. gallus

H. sapiens

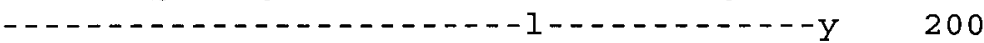

$X$. laevis

D. rerio

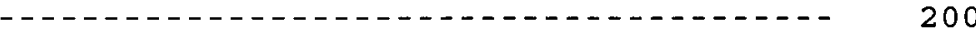

- - - v- - e-- - - - m---n---- - t-- - - - - - - 200

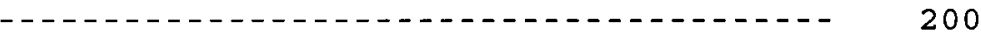

T. s. elegans

SDRGIPDGHRHMNGYG . . . . . . . . . . . . . 132

G. gallus

H. sapiens

$X$. laevis

D. rerio

- - - - - f- - - - shtfklvnasggavyckfhvktdq 240

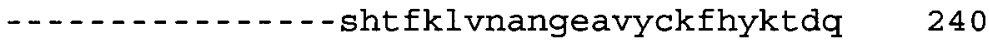

- - - - - - - - shtfklvsckdeavyckfhfktdq 240

- - - - - - - - - shtfklvnaqgqpvyckfhyktnq 240

FIG. 11. The partial amino acid sequence of catalse from $T$. s. elegans compared with catalase sequences from human (Homo sapiens), chicken (Gallus gallus), African clawed frog (Xenopus laevis) and zebrafish (Danio rerio). Accession numbers are CAG31019 for chicken, NP_001743 for human, NP_001088663 for frog and NP_570987 for zebrafish. Dashes replace amino acid residues identical with $T$. s. elegans while spacer dots indicate residues that are missing in the sequence. Turtle-specific amino acid substitution is bolded and shaded. 


\section{HDAC1}

T. S. elegans

G. gallus

$H$. sapiens

$X$. laevis

D. rerio

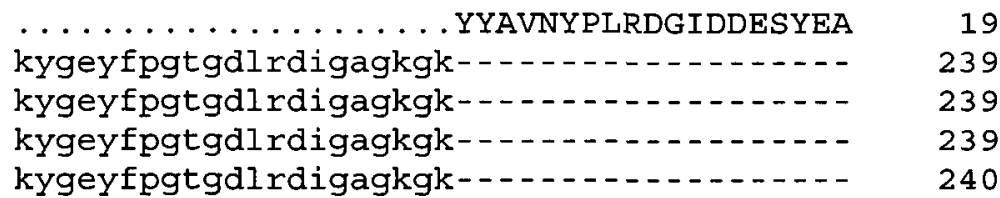

T. S. elegans

G. gallus

H. sapiens

$X$. laevis

D. rerio

T. s. elegans

G. gallus

H. sapiens

$X$. laevis

D. rerio

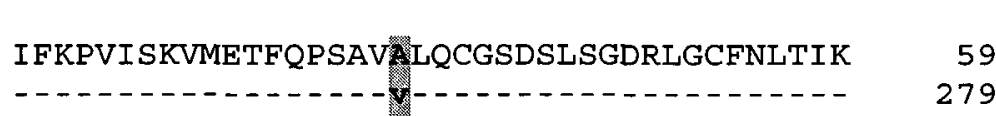

- - - m-

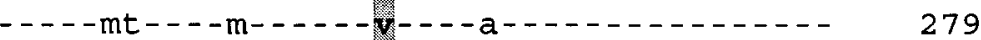

----im----my----1

T. s. elegans

GHAKCVEFVKSFNLPMLMLGGGGYTIRNVARCWTYETAVA 99

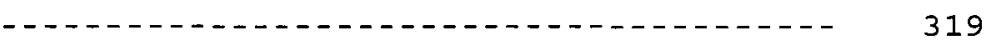

------------------------------ 319

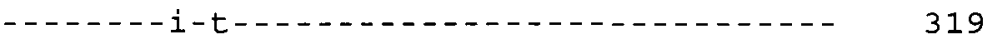

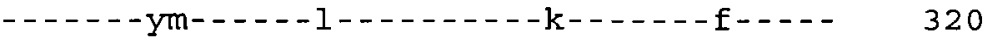

G. gallus

LDTEI PNELPYNDYFEYFGPDFKLHI SPSNMTNQNTNEYL 139

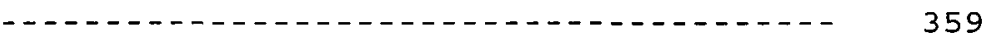

359

- S-D.- 359

$X$. laevis

D. rerio

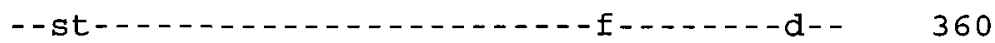

T. s. elegans

EKIKQR.......... 145

-----lfenlrmlphapgvq 399

-..--lfenlrmlphapgvq 399

----lfenlrmlphapgvg 399

H. sapiens

$X$. laevis

D. rerio

-..--lfenlrmlphapgrq

399

FIG. 12. The partial amino acid sequence of HDAC1 from $T$. $s$. elegans compared with HDAC1 sequences from human (Homo sapiens), chicken (Gallus gallus), African clawed frog (Xenopus laevis) and zebrafish (Danio rerio). Accession numbers are NP_989487 for chicken, NP_004955 for human, NP_001079396 for frog and NP_775343 for zebrafish. Dashes replace amino acid residues identical with T. $s$. elegans while spacer dots indicate residues that are missing in the sequence. Turtle-specific amino acid substitution is bolded and shaded. 


\section{HDAC2}

T. s. elegans

G. gallus

H. sapiens

$X$. laevis

T. S. elegans

G. gallus

H. sapiens

$X$. Laevis

T. s. elegans

G. gallus

H. sapiens

$X$. laevis

T. s. elegans

G. gallus

H. sapiens

$X$. laevis

T. s. elegans

G. gallus

H. sapiens

$X$. laevis

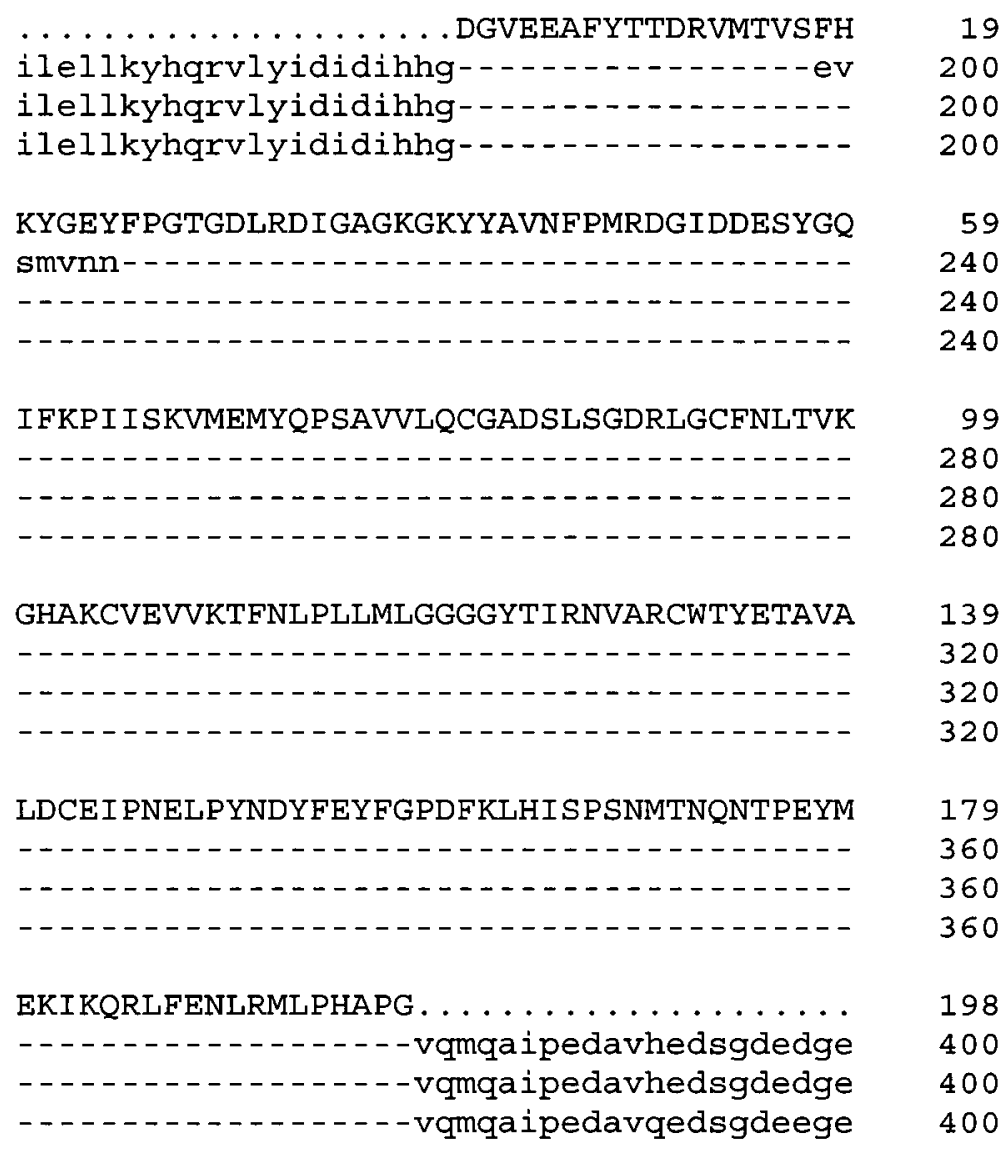

T. s. elegans

G. gallus

H. sapiens

$X$. laevis

FIG. 13. The partial amino acid sequence of HDAC2 from $T$. $s$. elegans compared with HDAC2 sequences from human (Homo sapiens), chicken (Gallus gallus) and African clawed frog (Xenopus laevis). Accession numbers are NP_990162 for chicken, NP_001518 for human and NP_001084011 for frog. Dashes replace amino acid residues identical with $T$. s. elegans while spacer dots indicate residues that are missing in the sequence. 


\section{HDAC3}

T. s. elegans

G. gallus

H. sapiens

$X$. laevis

$D$. rerio

.......... gaesgryyalnvpgaesgryyclnvpgaesgryyclnvpgaesgryyclnvp-

T. S. elegans

223

G. gallus

$H$. sapiens

$X$. laevis

D. rerio

RDGIDDQSYKHLFQPVINQVVDYYQPTCIVLQCGADSLGC $\quad 41$

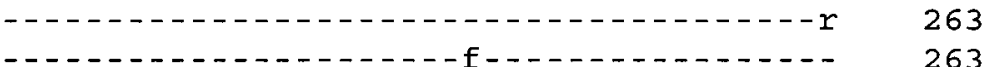

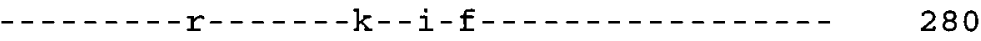

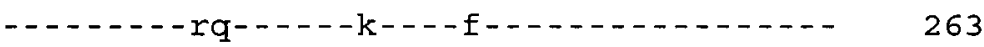

T. s. elegans

DRLGCFNLSIRGHGECVEYVKSFNI PLLVLGGGGYTVRNV 81

G. gallus

H. sapiens

303

$X$. laevis

-

D. rerio

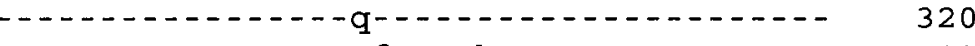

T. s. elegans

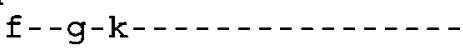

303

G. gallus

H. sapiens

ARCWTYETSLLVDEAISEELPYSEYFEYFAPDFTLHPDVS 121

$X$. laevis

D. rerio

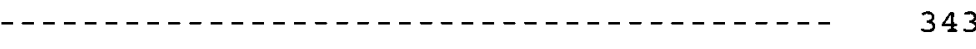

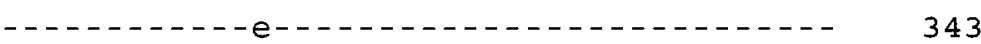

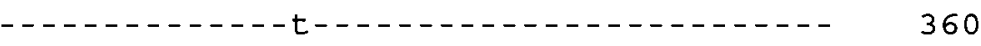

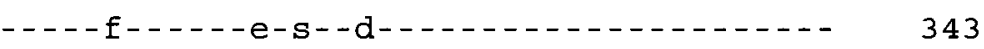

T. S. elegans

TRIENQNSRQYLDQIRQTIFENLKMLNHAP . . . . . . 151

G. gallus

H. sapiens

$X$. laevis

D. rerio

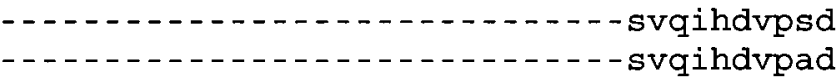

FIG. 14. The partial amino acid sequence of HDAC3 from $T$. s. elegans compared with HDAC3 sequences from human (Homo sapiens), chicken (Gallus gallus), African clawed frog (Xenopus laevis) and zebrafish (Danio rerio). Accession numbers are NP_990078 for chicken, NP_003874 for human, AAH70873 for frog and NP_957284 for zebrafish. Dashes replace amino acid residues identical with $T$. s. elegans while spacer dots indicate residues that are missing in the sequence. 


\section{HDAC4}

T. S. elegans

G. gallus

H. sapiens

D. rerio

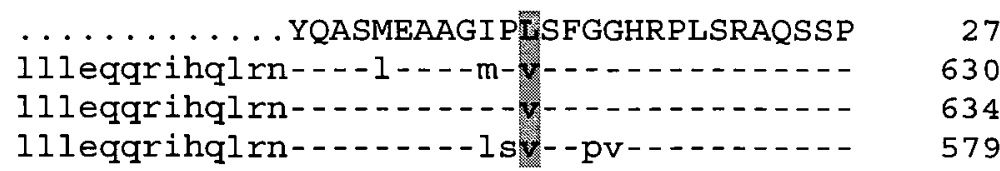

T. s. elegans

ASATFPMSVQEPPTKPRFTTGLVYDTLMLKHQCTCGNTNS 67

G. gallus

H. sapiens

A

D. rerio

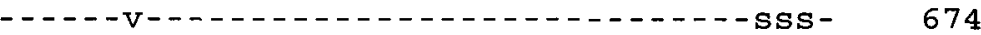

T. S. elegans

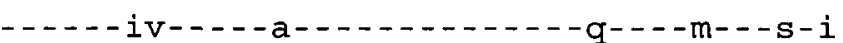

619

G. gallus

H. sapiens

HPEHAGRIQSIWS .............

D. rerio

FIG. 15. The partial amino acid sequence of HDAC4 from T. s. elegans compared with HDAC4 sequences from human (Homo sapiens), chicken (Gallus gallus) and zebrafish (Danio rerio). Accession numbers are NP_989644 for chicken, NP_006028 for human and NP_001034447 for zebrafish. Dashes replace amino acid residues identical with $T$. $s$. elegans while spacer dots indicate residues that are missing in the sequence. Turtlespecific amino acid substitution is bolded and shaded. 


\section{HDAC5}

T. S. elegans

H. sapiens

D. rerio

T. S. elegans

H. sapiens

D. rerio

T. s. elegans

$H$. sapiens

D. rerio

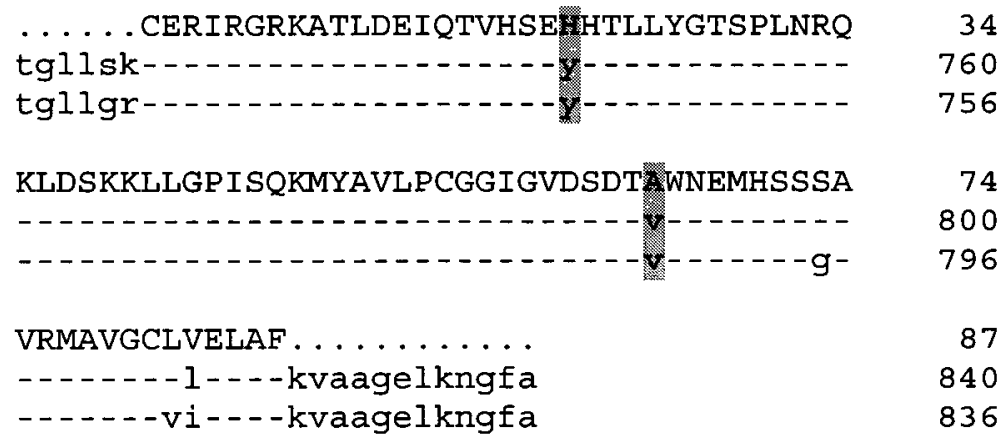

FIG. 16. The partial amino acid sequence of HDAC5 from $T$. s. elegans compared with HDAC5 sequences from human (Homo sapiens) and zebrafish (Danio rerio). Accession numbers are NP_005465 for human and XP_685659 for zebrafish. Dashes replace amino acid residues identical with $T$. s. elegans while spacer dots indicate residues that are missing in the sequence. Turtle-specific amino acid substitutions are bolded and shaded. 


\section{Appendix E: Ensuring antibody specificity for western blotting}

Throughout this thesis, several steps were taken to ensure that the correct band was quantified for western blotting. These include:

1. Blocking with milk before probing with the antibody was optimized to minimize any non-specific binding and ensure that only one band appeared at the expected molecular weight.

2. Most blots were run with a mammalian control homogenate in addition to the turtle samples to ensure that bands cross-react at the same molecular weight.

3. In case where antibodies were tested on several different animals by the company, antibodies that were shown to cross-react well with a wide number of different species were selected. For example, the histone $\mathrm{H} 3$ antibody used cross-reacted with animals ranging from mammals to fruit flies.

4. If antibodies for different regions of the protein were available from the company, the amino acid sequences of these regions were aligned with sequences from several vertebrates and antibodies specific to the best conserved regions were selected.

5. In cases where a partial turtle sequence was available prior to western blotting from PCR experiments, the translated turtle sequence was aligned with the sequence of the epitope the antibody was made against to assess conservation. Antibodies made from regions well-conserved with the turtle sequence were selected. 


\section{Appendix F: Examples of constantly expressed proteins in $T$. s. elegans tissues}

\begin{tabular}{|l|l|}
\hline Tissue & Proteins \\
\hline Heart & Actin, GSTK1, GST P1 \\
\hline Kidney & MnSOD, GST P1, GST T1, GST M3 \\
\hline Liver & MnSOD, GST M3, Nrf2, MCL-1 \\
\hline Muscle & Actin, Myosin, BCL-XL \\
\hline
\end{tabular}


REFERENCES 
Abraham NG, Kappas A. Pharmacological and clinical aspects of heme oxygenase. Pharmacol Rev 2008; 60(1):79-127.

Alessi DR, James SR, Downes CP, Holmes AB, Gaffney PR, Reese CB, Cohen P. Characterization of a 3-phosphoinositide-dependent protein kinase which phosphorylates and activates protein kinase B alpha. Curr Biol. 1997;7(4):2619.

Allert S, Ernest I, Poliszczak A, Opperdoes FR, Michels PA. Molecular cloning and analysis of two tandemly linked genes for pyruvate kinase of Trypanosoma brucei. Eur J Biochem. 1991;200(1):19-27.

Allfrey VG, Faulkner R, Mirsky AE. Acetylation and methylation of histones and their possible role in the regulation of RNA synthesis. Proc Natl Acad Sci USA 1964; 51:786-794.

Ameri K, Hammond EM, Culmsee C, Raida M, Katschinski DM, Wenger RH, Wagner E, Davis RJ, Hai T, Denko N, Harris AL. Induction of activating transcription factor 3 by anoxia is independent of $\mathrm{p} 53$ and the hypoxic HIF signalling pathway. Oncogene. 2007;26(2):284-9.

Anderson MJ, Viars CS, Czekay S, Cavenee WK, Arden KC. Cloning and characterization of three human forkhead genes that comprise an FKHR-like gene subfamily. Genomics. 1998;47(2):187-99.

Arden KC. FoxO: linking new signaling pathways. Mol Cell. 2004;14(4):416-8.

Assimacopoulos-Jeannet F, Jeanrenaud B. Insulin activates 6-phosphofructo-2-kinase and pyruvate kinase in the liver. Indirect evidence for an action via a phosphatase. $\mathrm{J}$ Biol Chem. 1990;265(13):7202-6.

Bakker WJ, Harris IS, Mak TW. FOXO3a is activated in response to hypoxic stress and inhibits HIF1-induced apoptosis via regulation of CITED2. Mol Cell. 2007;28(6):941-53.

Barrier A, Olaya N, Chiappini F, Roser F, Scatton O, Artus C, Franc B, Dudoit S, Flahault A, Debuire B, Azoulay D, Lemoine A. Ischemic preconditioning modulates the expression of several genes, leading to the overproduction of IL$1 \mathrm{Ra}$, iNOS, and Bcl-2 in a human model of liver ischemia-reperfusion. FASEB J 2005;19(12), 1617-26.

Barros SP, Offenbacher S. Epigenetics: connecting environment and genotype to phenotype and disease. J Dent Res. 2009;88(5):400-408.

Barthel A, Schmoll D, Unterman TG. FoxO proteins in insulin action and metabolism. Trends Endocrinol Metab. 2005;16(4):183-9. 
Bennin DA, Don AS, Brake T, McKenzie JL, Rosenbaum H, Ortiz L, DePaoli-Roach AA, Horne MC. Cyclin G2 associates with protein phosphatase 2A catalytic and regulatory B' subunits in active complexes and induces nuclear aberrations and a G1/S phase cell cycle arrest. J Biol Chem. 2002;277(30):27449-67.

Berberat PO, Katori M, Kaczmarek E, Anselmo D, Lassman C, Ke B, Shen X, Busuttil RW, Yamashita K, Csizmadia E, Tyagi S, Otterbein LE, Brouard S, Tobiasch E, Bach FH, Kupiec-Weglinski JW, Soares MP. Heavy chain ferritin acts as an antiapoptotic gene that protects livers from ischemia reperfusion injury. FASEB J. 2003;17(12):1724-6.

Berenshtein E, Vaisman B, Goldberg-Langerman C, Kitrossky N, Konijn AM, Chevion $\mathrm{M}$. Roles of ferritin and iron in ischemic preconditioning of the heart. Mol Cell Biochem. 2002;234-235(1-2):283-92.

Beresewicz A, Czarnowska E, Maczewski M. Ischemic preconditioning and superoxide dismutase protect against endothelial dysfunction and endothelium glycocalyx disruption in the postischemic guinea-pig hearts.Mol Cell Biochem. 1998;186(1-2):87-97.

Bergot MO, Diaz-Guerra MJ, Puzenat N, Raymondjean M, Kahn A. Cis-regulation of the L-type pyruvate kinase gene promoter by glucose, insulin and cyclic AMP. Nucleic Acids Res. 1992;20(8):1871-7.

Bertolotti, A., Zhang, Y., Hendershot, L. M., Harding, H. P. and Ron,D. Dynamic interaction of $\mathrm{BiP}$ and $\mathrm{ER}$ stress transducers in the unfolded-protein response. Nat. Cell Biol. 2000; 2:326-332.

Bickler PE, Buck LT. Hypoxia tolerance in reptiles, amphibians, and fishes: life with variable oxygen availability. Annu Rev Physiol 2007; 69:145-170.

Bickler PE, Donohoe PH, Buck LT. The hypoxic brain: suppressing energy-expensive membrane functions by regulation of receptors and ion channels. In Molecular Mechanisms of Metabolic Arrest (ed. Storey KB), Oxford: BIOS Scientific Publishers, 2001: 77-102.

Biel M, Wascholowski V, Giannis A. Epigenetics--an epicenter of gene regulation: histones and histone-modifying enzymes. Angew Chem Int Ed Engl. 2005;44(21):3186-216.

Bocharova LS, Gordon RY, Arkhipov V I. Uridine uptake and RNA synthesis in the brain of torpid and awakened ground squirrels. Comp Biochem Physiol B 1992; 101:189-192.

Bolli R, Marban E. Molecular and cellular mechanisms of myocardial stunning. Physiol Rev. 1999;79:609-634. 
Borkhardt A, Repp R, Haas OA, Leis T, Harbott J, Kreuder J, Hammermann J, Henn T, Lampert F. Cloning and characterization of AFX, the gene that fuses to MLL in acute leukemias with a $t(X ; 11)(q 13 ; q 23)$. Oncogene. 1997;14(2):195-202.

Boveris A, Chance B. The mitochondrial generation of hydrogen peroxide. Biochem $\mathbf{J}$ $1973 ; 134: 707-716$.

Brehm A, Miska EA, McChance DJ, Reid JL, Bannister AJ, Kouzarides T (1998) Retinoblastoma protein recruits histone deacetylase to repress transcription. Nature 391:597-601.

Brewer JW, Diehl JA. PERK mediates cell-cycle exit during the mammalian unfolded protein response. Proc Natl Acad Sci U S A. 2000;97(23):12625-30.

Brewer JW, Hendershot LM, Sherr CJ, Diehl JA. Mammalian unfolded protein response inhibits cyclin D1 translation and cell-cycle progression. Proc Natl Acad Sci U S A. 1999;96(15):8505-10.

Brooks SP, Storey KB. Regulation of glycolytic enzymes during anoxia in the turtle Pseudemys scripta. Am J Physiol. 1989;257(2 Pt 2):R278-83.

Brown SW. Heterochromatin. Science. 1666;151:417-425.

Brownawell AM, Kops GJ, Macara IG, Burgering BM. Inhibition of nuclear import by protein kinase $\mathrm{B}(\mathrm{Akt})$ regulates the subcellular distribution and activity of the forkhead transcription factor AFX. Mol Cell Biol. 2001;21:3534-3546.

Brunelle JK, Chandel NS. Oxygen deprivation induced cell death: an update. Apoptosis. 2002;7(6), 475-82.

Brunet A, Bonni A, Zigmond MJ, Lin MZ, Juo P, Hu LS, Anderson MJ, Arden KC, Blenis J, Greenberg ME. Akt promotes cell survival by phosphorylating and inhibiting a Forkhead transcription factor. Cell. 1999;96(6):857-68.

Brunet A, Kanai F, Stehn J, Xu J, Sarbassova D, Frangioni JV, Dalal SN, DeCaprio JA, Greenberg ME, Yaffe MB. 14-3-3 transits to the nucleus and participates in dynamic nucleocytoplasmic transport. J Cell Biol. 2002;156(5):817-28.

Brunet A, Sweeney LB, Sturgill JF, Chua KF, Greer PL, Lin Y, Tran H, Ross SE, Mostoslavsky R, Cohen HY, Hu LS, Cheng HL, Jedrychowski MP, Gygi SP, Sinclair DA, Alt FW, Greenberg ME. Stress-dependent regulation of FOXO transcription factors by the SIRT1 deacetylase. Science. 2004;303(5666):20115 .

Brush MH, Weiser DC, Shenolikar S. Growth arrest and DNA damage-inducible protein GADD34 targets protein phosphatase 1 alpha to the endoplasmic reticulum and promotes dephosphorylation of the alpha subunit of eukaryotic translation initiation factor 2. Mol Cell Biol. 2003;23(4):1292-303. 
Buck LT, Land SC, Hochachka PW. Anoxia-tolerant hepatocytes: model system for study of reversible metabolic suppression. Am J Physiol. 1993;265(1 Pt 2):R49-56.

Burgering BM, Coffer PJ. Protein kinase B (c-Akt) in phosphatidylinositol-3-OH kinase signal transduction. Nature. 1995;376:599-602.

Burgering BM, Kops GJ. Cell cycle and death control: long live Forkheads. Trends Biochem Sci. 2002;27(7):352-60.

Burke LJ, Baniahmad A. Co-repressors 2000. FASEB J. 2000;14:1876-1888.

Cadenas E, Boveris A, Ragan CI, Stoppani AO. Production of superoxide radicals and hydrogen peroxide by NADH-ubiquinone reductase and ubiquinol-cytochrome c reductase from beef-heart mitochondria. Arch Biochem Biophys 1977; 180:248-257.

Calfon M, Zeng H, Urano F, Till JH, Hubbard SR, Harding HP, Clark SG, Ron D. IRE1 couples endoplasmic reticulum load to secretory capacity by processing the XBP-1 mRNA. Nature. 2002;415(6867):92-6.

Calnan DR, Brunet A. The FoxO code. Oncogene. 2008;27(16):2276-88.

Calvanese V, Lara E, Kahn A, Fraga MF. The role of epigenetics in aging and age-related diseases. Ageing Res Rev. 2009; 8(4):268-276.

Catz SD, Johnson JL. Transcriptional regulation of bcl-2 by nuclear factor kappa B and its significance in prostate cancer. Oncogene. 2001;20(50):7342-51.

Chang J, Knowlton AA, Wasser JS. Expression of heat shock proteins in turtle and mammal hearts: relationship to anoxia tolerance. Am J Physiol 2000; 278(1):R209-R214.

Chen F, Demers LM, Vallyathan V, Lu Y, Castranova V, Shi X. Involvement of 5'flanking kappaB-like sites within bcl-x gene in silica-induced Bcl-x expression. J Biol Chem. 1999;274(50):35591-5.

Chen FE, Ghosh G. Regulation of DNA binding by Rel/NF-kappaB transcription factors: structural views. Oncogene.1999;18:6845-6852.

Chicoine LG, Schulman IG, Richman R, Cook RG, Allis CD. Nonrandom utilization of acetylation sites in histones isolated from Tetrahymena. Evidence for functionally distinct H4 acetylation sites. J Biol Chem. 1986;261(3):10711076.

Churchill TA. Organ preservation for transplantation, in: K.B. Storey (Ed.), Functional Metabolism: Regulation and Adaptation. Hoboken, NJ: Wiley-Liss, 2004: 383413. 
Connor JH, Weiser DC, Li S, Hallenbeck JM, Shenolikar S. Growth arrest and DNA damage-inducible protein GADD34 assembles a novel signaling complex containing protein phosphatase 1 and inhibitor 1. Mol Cell Biol. 2001;21(20):6841-50.

Cordis GA, Maulik G, Bagchi D, Riedel W, Das DK. Detection of oxidative DNA damage to ischemic reperfused rat hearts by 8 -hydroxydeoxyguanosine formation. J Mol Cell Cardiol. 1998;30:1939-1944.

Cordis GA, Maulik N, Das DK. Detection of oxidative stress in heart by estimating the dinitrophenyl-hydrazine derivative of malonaldehyde. J Mol Cell Cardiol.1995; 27:1645-1653.

Cullinan SB, Diehl JA. PERK-dependent activation of Nrf2 contributes to redox homeostasis and cell survival following endoplasmic reticulum stress. J Biol Chem. 2007;279(19):20108-17.

Cullinan SB, Zhang D, Hannink M, Arvisais E, Kaufman RJ, Diehl JA. Nrf2 is a direct PERK substrate and effector of PERK-dependent cell survival.Mol Cell Biol. 2003;23(20):7198-209.

Cummins EP, Taylor CT. Hypoxia-responsive transcription factors. Pflugers Arch. 2005;450(6):363-71.

Dansen TB, Kops GJ, Denis S, Jelluma N, Wanders RJ, Bos JL, Burgering BM, Wirtz $\mathrm{KW}$. Regulation of sterol carrier protein gene expression by the forkhead transcription factor FOXO3a. J Lipid Res. 2004;45(1):81-8.

Das, K.C., Lewis-Molock, Y., White, C.W., 1995. Activation of NF-kappa B and elevation of MnSOD gene expression by thiol reducing agents in lung adenocarcinoma (A549) cells. Am. J. Physiol. 269(5 Pt 1), L588-602.

Dawson TL, Gores GJ, Nieminen AL, Herman B, Lemasters J. Mitochondria as a source of reactive oxygen species during reductive stress in rat hepatocytes. Am J Physiol Cell Physiol.1993; 264, C961-C967.

Dentin R, Benhamed F, Pégorier JP, Foufelle F, Viollet B, Vaulont S, Girard J, Postic C. Polyunsaturated fatty acids suppress glycolytic and lipogenic genes through the inhibition of ChREBP nuclear protein translocation. J Clin Invest. 2005;115(10):2843-54.

Dentin R, Pégorier JP, Benhamed F, Foufelle F, Ferré P, Fauveau V, Magnuson MA, Girard J, Postic C. Hepatic glucokinase is required for the synergistic action of ChREBP and SREBP-1c on glycolytic and lipogenic gene expression. J Biol Chem. 2004;279(19):20314-26. 
Dignam JD, Lebovitz RM, Roeder RG. Accurate transcription initiation by RNA polymerase II in a soluble extract from isolated mammalian nuclei. Nucleic Acids Res. 1983;11(5):1475-89.

Dinkelacker SA, Costanzo JP, Lee RE Jr. Anoxia tolerance and freeze tolerance in hatchling turtles. J Comp Physiol B 2005;175(3):209-217.

Doi K, Akaike T, Fujii S, Tanaka S, Ikebe N, Beppu T, Shibahara S, Ogawa M, Maeda $\mathrm{H}$. Induction of haem oxygenase-1 nitric oxide and ischaemia in experimental solid tumours and implications for tumour growth. Br J Cancer 1999; 80:19451954.

Dorner, A. J., Wasley, L. C. and Kaufman, R. J. Overexpression of GRP78 mitigates stress induction of glucose regulated proteins and blocks secretion of selective proteins in Chinese hamster ovary cells. EMBO J. 1992; 11:1563-1571.

Ellgaard L, Ruddock LW. The human protein disulphide isomerase family: substrate interactions and functional properties. EMBO Rep. 2005;6(1):28-32.

El-Maghrabi MR, Haston WS, Flockhart DA, Claus TH, Pilkis SJ. Studies on the phosphorylation and dephosphorylation of L-type pyruvate kinase by the catalytic subunit of cyclic AMP-dependent protein kinase. J Biol Chem. 1980;255(2):668-75.

Essers MA, de Vries-Smits LM, Barker N, Polderman PE, Burgering BM, Korswagen HC. Functional interaction between beta-catenin and FOXO in oxidative stress signaling. Science. 2005;308(5725):1181-4.

Fan C, Li Q, Zhang Y, Liu X, Luo M, Abbott D, Zhou W, Engelhardt JF. IkappaBalpha and IkappaBbeta possess injury context-specific functions that uniquely influence hepatic NF-kappaB induction and inflammation.J Clin Invest. 2004;113(5):746-55.

Fels DR, Koumenis C. The PERK/eIF2alpha/ATF4 module of the UPR in hypoxia resistance and tumor growth. Cancer Biol Ther. 2006;5(7):723-8.

Fischle W, Dequiedt F, Hendzel MJ, Guenther MG, Lazar MA, Voelter W, Verdin E. Enzymatic activity associated with class II HDACs is dependent on a multiprotein complex containing HDAC3 and SMRT/N-CoR. Mol Cell. 2002; 9:45-57.

Fischle W, Emiliani S, Hendzel MJ, Nagase T, Nomura N, Voelter W, Verdin E. A new family of human histone deacetylases related to Saccharomyces cerevisiae HDAlp. J Biol Chem. 1999;274:11713-11720.

Franke TF, Yang SI, Chan TO, Datta K, Kazlauskas A, Morrison DK, Kaplan DR, Tsichlis PN. The protein kinase encoded by the Akt proto-oncogene is a target of the PDGF-activated phosphatidylinositol 3-kinase. Cell. 1995;81(5):727-36. 
Fraser K P, Houlihan D F, Lutz PL, Leone-Kabler S, Manuel L, Brechin JG. Complete suppression of protein synthesis during anoxia with no post-anoxia protein synthesis debt in the red-eared slider turtle Trachemys scripta elegans. J Exp Biol 2001; 204:4353-60.

Frescas D, Valenti L, Accili D. Nuclear trapping of the forkhead transcription factor FoxOl via Sirt-dependent deacetylation promotes expression of glucogenetic genes. J Biol Chem. 2005; 280(21):20589-95.

Friedlander, R., Jarosch, E., Urban, J., Volkwein, C. and Sommer, T. A regulatory link between ER-associated protein degradation and the unfolded-protein response. Nat Cell Biol .2000; 2:379-384.

Galili N, Davis RJ, Fredericks WJ, Mukhopadhyay S, Rauscher FJ 3rd, Emanuel BS, Rovera G, Barr FG. Fusion of a fork head domain gene to PAX3 in the solid tumour alveolar rhabdomyosarcoma. Nat Genet. 1993;5(3):230-5.

Glozak MA, Sengupta N, Zhang X, Seto E. Acetylation and deacetylation of non-histone proteins. Gene. 2005; 363:15-23.

Guo S, Rena G, Cichy S, He X, Cohen P, Unterman T. Phosphorylation of serine 256 by protein kinase B disrupts transactivation by FKHR and mediates effects of insulin on insulin-like growth factor-binding protein-1 promoter activity through a conserved insulin response sequence. J Biol Chem. 1999;274(24):17184-92.

Gupta S. Molecular steps of death receptor and mitochondrial pathways of apoptosis. Life Sci 2001; 69:2954-2957.

Hai T. The ATF transcription factors in cellular adaptive responses. In: Ma J (ed) Gene expression and regulation. 2005. Higher Education Press, Beijing, China, pp 322-333.

Halliwell B. Biochemistry of oxidative stress. Biochem Soc Trans 2007; 35(Pt 5):11471150.

Hamdane M, David-Cordonnier MH, D'Halluin JC. Activation of p65 NF-kappaB protein by $\mathrm{p} 210 \mathrm{BCR}-\mathrm{ABL}$ in a myeloid cell line (P210BCR-ABL activates p65 NFkappaB).Oncogene. 1997;15(19):2267-75.

Harding HP, Zhang Y, Bertolotti A, Zeng H, Ron D. Perk is essential for translational regulation and cell survival during the unfolded protein response. Mol Cell. 2000;5:897-904.

Harding HP, Zhang Y, Ron D. Protein translation and folding are coupled by an endoplasmicreticulum- resident kinase. Nature. 1999;397:271-274. 
Harding HP, Zhang Y, Zeng H, Novoa I, Lu PD, Calfon M, Sadri N, Yun C, Popko B, Paules R, Stojdl DF, Bell JC, Hettmann T, Leiden JM, Ron D. An integrated stress response regulates amino acid metabolism and resistance to oxidative stress. Mol Cell. 2003;11(3):619-33.

Haze K, Yoshida H, Yanagi H, Yura T, Mori K. Mammalian transcription factor ATF6 is synthesized as a transmembrane protein and activated by proteolysis in response to endoplasmic reticulum stress. Mol Biol Cell 1999;10:3787-3799.

Hebbes TR, Thorne AW, Crane-Robinson C. A direct link between core histone acetylation and transcriptionally active chromatin. EMBO J 1988; 7(5):13951402 .

Hemmings SJ, Storey KB. Hepatic changes in the freeze-tolerant turtle Chrysemys picta marginata in response to freezing and thawing. Cell Biochem Funct 2000;18(3):175-186.

Henderson ST, Johnson TE. Daf-16 integrates developmental and environmental inputs to mediate aging in the nematode Caenorhabditis elegans. Curr Biol. $2001 ; 11(24): 1975-80$.

Hentze MW, Muckenthaler MU, Andrews NC. Balancing acts: molecular control of mammalian iron metabolism. Cell. 2004; 117:285 -297.

Herbert CV, Jackson DC. Temperature effects on the responses to prolonged submergence in the turtle Chrysemys picta bellii. II. Metabolic rate, blood-acid base and ionic changes, and cardiovascular function in aerated and anoxic water. Physiol Zool. 1985; 58:670-681.

Hermes-Lima M, Storey JM, Storey KB. Antioxidant defenses and animal adaptation to oxygen availability during environmental stress. In: Cell and Molecular Responses to Stress (Storey, K.B. and Storey, J.M., eds.), Elsevier Press, Amsterdam, 2001; Vol. 2: pp. 263-287.

Hermes-Lima M, Storey KB. Antioxidant defenses and metabolic depression in a pulmonate land snail. Am J Physiol. 1995;268(6 Pt 2):R1386-93.

Hermes-Lima M, Storey KB. Antioxidant defenses in the tolerance of freezing and anoxia by garter snakes. Am J Physiol. 1993;265(3 Pt 2):R646-52.

Hermes-Lima M. Oxygen in biology and biochemistry: role of free radicals. In: K.B. Storey (Ed.), Functional Metabolism: Regulation and Adaptation. Hoboken, NJ: Wiley-Liss, 2004: 319-368.

Hillion J, Le Coniat M, Jonveaux P, Berger R, Bernard OA. AF6q21, a novel partner of the MLL gene in $\mathrm{t}(6 ; 11)(\mathrm{q} 21 ; \mathrm{q} 23)$, defines a forkhead transcriptional factor subfamily. Blood. 1997;90(9):3714-9. 
Ho A, Dowdy SF. Regulation of G(1) cell-cycle progression by oncogenes and tumor suppressor genes. Curr Opin Genet Dev. 2002;12(1):47-52.

Hochachka PW, Buck LT, Doll CJ, Land SC. Unifying theory of hypoxia tolerance: molecular/metabolic defense and rescue mechanisms for surviving oxygen lack. Proc Natl Acad Sci U S A. 1996;93(18):9493-8.

Hochachka PW, Lutz PL. Mechanism, origin and evolution of anoxia tolerance in animals. Comp Biochem Physiol 2001; 130B:435-459.

Hollander JM, Lin KM, Scott BT, Dillmann WH. Overexpression of PHGPx and HSP60/10 protects against ischemia/reoxygenation injury. Free Radic Biol Med 2003; 35(7):742-751.

Honda Y, Honda S. The daf-2 gene network for longevity regulates oxidative stress resistance and Mn-superoxide dismutase gene expression in Caenorhabditis elegans. FASEB J. 1999;13(11):1385-93

Horne MC, Donaldson KL, Goolsby GL, Tran D, Mulheisen M, Hell JW, Wahl AF. Cyclin G2 is up-regulated during growth inhibition and B cell antigen receptormediated cell cycle arrest. J Biol Chem. 1997;272(19):12650-61.

$\mathrm{Hu} \mathrm{GH}$, Lu XS .Effect of normothermic liver ischemic preconditioning on the expression of apoptosis-regulating genes $\mathrm{C}-\mathrm{jun}$ and $\mathrm{Bcl}-\mathrm{XL}$ in rats. World $\mathrm{J}$ Gastroenterol. 2005; 11(17):2579-82.

Hudson NJ, Lonhienne TG, Franklin CE, Harper GS, Lehnert SA. Epigenetic silencers are enriched in dormant desert frog muscle. J Comp Physiol B. 2008; 178:729734.

Idriss NK, Blann AD, Lip GY. Hemoxygenase-1 in cardiovascular disease. J Am Coll Cardiol 2008; 52(12):971-978.

Imamura, K., Noguchi, T. and Tanaka, T. Regulation of isozyme patterns of pyruvate kinase in normal and neoplastic tissues. 1986. In: Markers of Human Neuroectodermal Tumors (Staal, G. E. J. and van Veelen, C. W. M., eds.), pp. 191-222, CRC Press, Boca Raton, FL

Ishibashi H, Cottam GL. Glucagon-stimulated phosphorylation of pyruvate kinase in hepatocytes. J Biol Chem. 1978;253(24):8767-71.

Ishii S, Iizuka K, Miller BC, Uyeda K. Carbohydrate response element binding protein directly promotes lipogenic enzyme gene transcription. Proc Natl Acad Sci U S A. 2004;101:15597-602.

Jackson DC. Living without oxygen: lessons from the freshwater turtle. Comp Biochem Physiol A Mol Integr Physiol. 2000;125(3):299-315. 
Jackson DC, Heisler N. Intracellular and extracellular acid-base and electrolyte status of submerged anoxic turtles at $3^{\circ} \mathrm{C}$. Respir Physiol. 1983; 53:187-201

Jacobs FM, van der Heide LP, Wijchers PJ, Burbach JP, Hoekman MF, Smidt MP. FoxO6, a novel member of the FoxO class of transcription factors with distinct shuttling dynamics. J Biol Chem. 2003;278(38):35959-67.

Janumyan YM, Sansam CG, Chattopadhyay A, Cheng N, Soucie EL, Penn LZ, Andrews D, Knudson CM, Yang E. Bcl-XL/Bcl-2 coordinately regulates apoptosis, cell cycle arrest and cell cycle entry. EMBO J 2003; 22:5459-5470.

Jiang HY, Wek SA, McGrath BC, Lu D, Hai T, Harding HP, Wang X, Ron D, Cavener $\mathrm{DR}$, Wek RC. Activating transcription factor 3 is integral to the eukaryotic initiation factor 2 kinase stress response. Mol Cell Biol. 2004;24(3):1365-77.

Johnson CA, Turner BM. Histone deacetylases: complex transducers of nuclear signals. Semin Cell Dev Biol. 1999;10:179-188.

Jones P, Altamura S, De Francesco R, Gallinari P, Lahm A, Neddermann P, Rowley M, Serafini S, Steinkühler C. Probing the elusive catalytic activity of vertebrate class IIa histone deacetylases. Bioorg Med Chem Lett. 2008;18:1814-1819.

Kabashima T, Kawaguchi T, Wadzinski BE, Uyeda K. Xylulose 5-phosphate mediates glucose-induced lipogenesis by xylulose 5-phosphate-activated protein phosphatase in rat liver. Proc Natl Acad Sci U S A. 2003;100(9):5107-12.

Kalmar B, Greensmith L. Induction of heat shock proteins for protection against oxidative stress. Adv Drug Deliv Rev 2009; 61(4):310-318.

Karin M, Ben Neriah Y. Phosphorylation meets ubiquitination: the control of NF-kappaB activity. Annu Rev Immunol. 2000;18:621.

Karin M. How NF-kappaB is activated: the role of the IkappaB kinase (IKK) complex. Oncogene. 1999;18:6867-74.

Katic M, Kahn CR. The role of insulin and IGF-1 signaling in longevity. Cell Mol Life Sci. 2005;62(3):320-43.

Kaufman RJ. Regulation of mRNA translation by protein folding in the endoplasmic reticulum. Trends Biochem Sci 2004;29:152-158.

Kawaguchi T, Takenoshita M, Kabashima T, Uyeda K. Glucose and cAMP regulate the L-type pyruvate kinase gene by phosphorylation/dephosphorylation of the carbohydrate response element binding protein. Proc Natl Acad Sci U S A. 2001;98(24):13710-5.

Kelly DA, Storey KB. Organ-specific control of glycolysis in anoxic turtles. Am J Physiol. 1988;255(5 Pt 2):R774-9. 
Kesaraju S, Schmidt-Kastner R, Prentice HM, Milton SL. Modulation of stress proteins and apoptotic regulators in the anoxia tolerant turtle brain. J Neurochem 2009; 109(5):1413-1426.

Kim MS, Kwon HJ, Lee YM, Baek JH, Jang JE, Lee SW, Moon EJ, Kim HS, Lee SK, Chung HY, Kim CW, Kim KW. Histone deacetylases induce angiogenesis by negative regulation of tumor suppressor genes. Nat Med. 2001;7:437-443

Kitamura YI, Kitamura T, Kruse JP, Raum JC, Stein R, Gu W, Accili D. FoxO1 protects against pancreatic beta cell failure through NeuroD and MafA induction. Cell Metab. 2005;2(3):153-63.

Kluck RM, Bossy-Wetzel E, Green DR, Newmeyer DD. The release of cytochrome c from mitochondria: a primary site for Bcl-2 regulation of apoptosis. Science 1997; 275:1132-1136.

Kobayashi Y, Furukawa-Hibi Y, Chen C, Horio Y, Isobe K, Ikeda K, Motoyama N. SIRT1 is critical regulator of FOXO-mediated transcription in response to oxidative stress. Int J Mol Med. 2005;16(2):237-43.

Kokame K, Kato H, Miyata T. Identification of ERSE-II, a new cis-acting element responsible for the ATF6-dependent mammalian unfolded protein response. J Biol Chem. 2001;276(12):9199-205.

Kops GJ, Medema RH, Glassford J, Essers MA, Dijkers PF, Coffer PJ, Lam EW, Burgering BM. Control of cell cycle exit and entry by protein kinase Bregulated forkhead transcription factors. Mol Cell Biol. 2002a;22(7):2025-36.

Kops GJ, Dansen TB, Polderman PE, Saarloos I, Wirtz KW, Coffer PJ, Huang TT, Bos JL, Medema RH, Burgering BM. Forkhead transcription factor FOXO3a protects quiescent cells from oxidative stress. Nature. 2002b;419(6904):31621.

Kozutsumi,Y., Segal, M.,Normington, K.,Gething,M.-J. and Sambrook, J. The presence of malfolded proteins in the endoplasmic reticulum signals the induction of glucose-regulated proteins. Nature. 1988; 332:462-464.

Krivoruchko A, Storey KB. Activation of antioxidant defenses in response to freezing in freeze tolerant painted turtle hatchlings. Biochim Biophys Acta 2010a; 1800(7):662-668.

Krivoruchko A, Storey KB. Regulation of the heat shock response under anoxia in the turtle, Trachemys scripta elegans. J Comp Physiol B 2010b; 180(3):403-414.

Krivoruchko A, Storey KB. Molecular mechanisms of turtle anoxia tolerance: A role for NF-kappaB. Gene. 2010c;450(1-2):63-9. 
Krivoruchko A, Storey KB. Epigenetics in anoxia tolerance: a role for histone deacetylases.Mol Cell Biochem. 2010d. Epub

Kuboki, S., Okaya, T., Schuster, R., Blanchard, J., Denenberg, A., Wong, H.R., Lentsch, A.B., 2007. Hepatocyte NF-kappaB activation is hepatoprotective during ischemia-reperfusion injury and is augmented by ischemic hypothermia. Am. J. Physiol. Gastrointest. Liver Physiol. 292(1), G201-7.

Kwak EL, Larochelle DA, Beaumont C, Torti SV, Torti FM. Role for NF-kappa B in the regulation of ferritin $\mathrm{H}$ by tumor necrosis factor-alpha. J Biol Chem. 1995;270(25):15285-93.

Laemmli UK. Cleavage of structural proteins during the assembly of the head of bacteriophage T4. Nature. 1970;227(5259):680-5.

Lahm A, Paolini C, Pallaoro M, Nardi MC, Jones P, Neddermann P, Sambucini S, Bottomley MJ, Lo Surdo P, Carfí A, Koch U, De Francesco R, Steinkühler C, Gallinari P. Unraveling the hidden catalytic activity of vertebrate class IIa histone deacetylases. Proc Natl Acad Sci U S A. 2007;104(44):17335-40.

Land SC, Buck LT, Hochachka PW. Response of protein synthesis to anoxia and recovery in anoxia-tolerant hepatocytes. Am J Physiol. 1993;265(1 Pt 2):R418.

Larade K, Storey KB. A profile of the metabolic responses to anoxia in marine invertebrates. In Sensing, Signaling and Cell Adaptation (ed. Storey KB and Storey JM), Amsterdam: Elsevier Science, 2002: 27-46.

Latanich CA, Toledo-Pereyra LH. Searching for NF-kappaB-based treatments of ischemia reperfusion injury. J Invest Surg 2009: 22(4):301-315.

Lee AH, Iwakoshi NN, Glimcher LH. XBP-1 regulates a subset of endoplasmic reticulum resident chaperone genes in the unfolded protein response. Mol Cell Biol. 2003;23(21):7448-59.

Lee AS. The glucose-regulated proteins: stress induction and clinical applications. Trends Biochem Sci. 2001 Aug;26(8):504-10.

Lee HH, Dadgostar H, Cheng Q, Shu J, Cheng G. NF-kappaB-mediated up-regulation of Bcl- $x$ and $\mathrm{Bfl}-1 / \mathrm{A} 1$ is required for CD40 survival signaling in $\mathrm{B}$ lymphocytes. Proc Natl Acad Sci U S A. 1999;96(16):9136-41.

Lee K, Tirasophon W, Shen X, Michalak M, Prywes R, Okada T, Yoshida H, Mori K, Kaufman RJ. IRE1-mediated unconventional mRNA splicing and S2Pmediated ATF6 cleavage merge to regulate XBP1 in signaling the unfolded protein response. Genes Dev. 2002;16(4):452-66. 
Lehtinen MK, Yuan Z, Boag PR, Yang Y, Villén J, Becker EB, DiBacco S, de la Iglesia N, Gygi S, Blackwell TK, Bonni A. A conserved MST-FOXO signaling pathway mediates oxidative-stress responses and extends life span. Cell. 2006;125(5):987-1001.

Li M, Baumeister P, Roy B, Phan T, Foti D, Luo S, Lee AS. ATF6 as a transcription activator of the endoplasmic reticulum stress element: thapsigargin stressinduced changes and synergistic interactions with NF-Y and YY1. Mol Cell Biol. 2000;20(14):5096-106.

Lin K, Dorman JB, Rodan A, Kenyon C. daf-16: An HNF-3/forkhead family member that can function to double the life-span of Caenorhabditis elegans. Science. 1997;278(5341):1319-22

Lindquist S, Craig EA. The heat-shock proteins. Annu Rev Genet 1988; 22:631-677.

Lipton P. Ischemic cell death in brain neurons. Physiol Rev. 1999;79:1431-1568.

$\mathrm{Lu} \mathrm{D}$, Wolfgang CD, Hai T. Activating transcription factor 3, a stress-inducible gene, suppresses Ras-stimulated tumorigenesis. J Biol Chem. 2006;281(15):1047381.

Lu J, McKinsey TA, Nicol RL, Olson EN. Signal-dependent activation of the MEF2 transcription factor by dissociation from histone deacetylases. Proc Natl Acad Sci USA. 2000;97:4070-4075.

Luedde T, Assmus U, Wüstefeld T, Meyer zu Vilsendorf A, Roskams T, SchmidtSupprian M, Rajewsky K, Brenner DA, Manns MP, Pasparakis M, Trautwein C. Deletion of IKK2 in hepatocytes does not sensitize these cells to TNFinduced apoptosis but protects from ischemia/reperfusion injury. J Clin Invest. 2005;115(4):849-59.

Luedde, T., Trautwein, C., 2006. Intracellular survival pathways in the liver. Liver. Int. 26(10), 1163-74.

Lutz PL, Nilsson GE. Vertebrate brains at the pilot light. Respir Physiol Neurobiol 2004;141:285-296.

Ma A, Qi S, Chen H. Antioxidant therapy for prevention of inflammation, ischemic reperfusion injuries and allograft rejection. Cardiovasc Hematol Agents Med Chem 2008; 6(1):20-43.

Ma Y, Hendershot LM. Delineation of a negative feedback regulatory loop that controls protein translation during endoplasmic reticulum stress. J Biol Chem. 2003;278(37):34864-73.

Ma Y, Hendershot LM. Herp is dually regulated by both the endoplasmic reticulum stress-specific branch of the unfolded protein response and a branch that is 
shared with other cellular stress pathways. J Biol Chem. 2004;279(14):137929.

Macaluso M, Montanari M, Cinti C, Giordano A. Modulation of cell cycle components by epigenetic and genetic events. Semin Oncol. 2005;32(5):452-457.

Martínez-Gac L, Marqués M, García Z, Campanero MR, Carrera AC. Control of cyclin G2 mRNA expression by forkhead transcription factors: novel mechanism for cell cycle control by phosphoinositide 3-kinase and forkhead. Mol Cell Biol. 2004;24(5):2181-9.

Matsui N, Kasajima K, Hada M, Nagata T, Senga N, Yasui Y, Fukuishi N, Akagi M. Inhibiton of NF-kappaB activation during ischemia reduces hepatic ischemia/reperfusion injury in rats. J Toxicol Sci. 2005;30(2):103-10.

Matsuzaki H, Daitoku H, Hatta M, Tanaka K, Fukamizu A. Insulin-induced phosphorylation of FKHR (Foxol) targets to proteasomal degradation. Proc Natl Acad Sci U S A. 2003;100(20):11285-90.

McClintock DS, Santore MT, Lee VY, Brunelle J, Budinger GR, Zong WX, Thompson CB, Hay N, Chandel NS. Bcl-2 family members and functional electron transport chain regulate oxygen deprivation-induced cell death. Mol Cell Biol. 2002;22(1):94-104.

McKinsey TA, Zhang CL, Lu J, Olson EN. Signal-dependent nuclear export of a histone deacetylase regulates muscle differentiation. Nature. 2000;408:106-111.

Medema RH, Kops GJ, Bos JL, Burgering BM. AFX-like Forkhead transcription factors mediate cell-cycle regulation by Ras and PKB through p27kip1.Nature. 2000;404(6779):782-7.

Mehrani H, Storey KB. Enzymatic control of glycogenolysis during anoxic submergence in the freshwater turtle Trachemys scripta. Int J Biochem Cell Biol. $1995 ; 27(8): 821-30$.

Minami K, Inoue H, Terashita T, Kawakami T, Watanabe R, Haneda M, Isobe K, Okabe $\mathrm{H}$, Chano T. GADD34 induces p21 expression and cellular senescence. Oncol Rep. 2007;17(6):1481-5.

Misra A, Haudek SB, Knuefermann P, Vallejo JG, Chen ZJ, Michael LH, Sivasubramanian N, Olson EN, Entman ML, Mann DL. Nuclear factor-kappaB protects the adult cardiac myocyte against ischemia-induced apoptosis in a murine model of acute myocardial infarction. Circulation. 2003;108(25):30758.

Morin P Jr, Ni Z, McMullen DC, Storey KB. Expression of Nrf2 and its downstream gene targets in hibernating 13-lined ground squirrels, Spermophilus tridecemlineatus. Mol Cell Biochem. 2008;312(1-2):121-9. 
Morin $\mathrm{P} \mathrm{Jr}$, Storey KB. Evidence for a reduced transcriptional state during hibernation in ground squirrels. Cryobiology. 2006;53(3):310-318.

Motterlini R, Foresti R, Bassi R, Calabrese V, Clark JE, Green CJ. Endothelial heme oxygenase-1 induction by hypoxia. Modulation by inducible nitric-oxide synthase and S-nitrosothiols. J Biol Chem 2000; 275:13613-13620.

Motterlini R, Foresti R, Intaglietta M, Winslow RM. NO-mediated activation of heme oxygenase: endogenous cytoprotection against oxidative stress to endothelium. Am J Physiol 1996; 270:H107-H114.

Nakamura N, Ramaswamy S, Vazquez F, Signoretti S, Loda M, Sellers WR. Forkhead transcription factors are critical effectors of cell death and cell cycle arrest downstream of PTEN. Mol Cell Biol. 2000;20(23):8969-82.

Nakao M. Epigenetics: interaction of DNA methylation and chromatin. Gene. $2001 ; 278(1-2): 25-31$

Nichols TC. NF-kappaB and reperfusion injury. Drug News Perspect. 2004;17(2):99-104.

Noguchi T, Yamada K, Yamagata K, Takenaka M, Nakajima H, Imai E, Wang Z, Tanaka T. Expression of liver type pyruvate kinase in insulinoma cells: involvement of LF-B1 (HNF1). Biochem Biophys Res Commun. 1991;181(1):259-64.

Novoa I, Zeng H, Harding HP, Ron D. Feedback inhibition of the unfolded protein response by GADD34-mediated dephosphorylation of eIF2alpha. J Cell Biol. 2001;153(5):1011-22.

Obolensky A, Berenshtein E, Konijn AM, Banin E, Chevion M. Ischemic preconditioning of the rat retina: protective role of ferritin. Free Radic Biol Med. 2008;44(7):1286-94.

Oda Y, Okada T, Yoshida H, Kaufman RJ, Nagata K, Mori K. Derlin-2 and Derlin-3 are regulated by the mammalian unfolded protein response and are required for ER-associated degradation. J Cell Biol. 2006; 172:383-393.

Ogg S, Paradis S, Gottlieb S, Patterson GI, Lee L, Tissenbaum HA, Ruvkun G. The Fork head transcription factor DAF-16 transduces insulin-like metabolic and longevity signals in C. elegans. Nature. 1997;389(6654):994-9.

Oh SW, Mukhopadhyay A, Svrzikapa N, Jiang F, Davis RJ, Tissenbaum HA. JNK regulates lifespan in Caenorhabditis elegans by modulating nuclear translocation of forkhead transcription factor/DAF-16. Proc Natl Acad Sci U S A. 2005;102(12):4494-9.

Okada T, Yoshida H, Akazawa R, Negishi M, Mori K. Distinct roles of activating transcription factor 6 (ATF6) and double-stranded RNA-activated protein 
kinase-like endoplasmic reticulum kinase (PERK) in transcription during the mammalian unfolded protein response. Biochem J. 2002;366(Pt 2):585-94.

Ollinger R, Wang H, Yamashita K, Wegiel B, Thomas M, Margreiter R, Bach FH. Therapeutic applications of bilirubin and biliverdin in transplantation. Antioxid Redox Signal 2007; 9(12):2175-85.

Onai Y, Suzuki J, Kakuta T, Maejima Y, Haraguchi G, Fukasawa H, Muto S, Itai A, Isobe $\mathrm{M}$. Inhibition of IkappaB phosphorylation in cardiomyocytes attenuates myocardial ischemia/reperfusion injury. Cardiovasc Res. 2004;63(1):51-9.

Ostojić S, Pereza N, Kapović M. A current genetic and epigenetic view on human aging mechanisms. Coll Antropol. 2009;33(2):687-699.

Pahl HL, Baeuerle PA. A novel signal transduction pathway from the endoplasmic reticulum to the nucleus is mediated by transcription factor NF-kappa B. EMBO J. 1995;14(11):2580-8.

Pahl HL. Activators and target genes of Rel/NF-kappaB transcription factors. Oncogene $1999 ; 18: 6853-6866$.

Papandreou I, Krishna C, Kaper F, Cai D, Giaccia AJ, Denko NC. Anoxia is necessary for tumor cell toxicity caused by a low-oxygen environment. Cancer Res. $2005 ; 65: 3171-3178$.

Paradis S, Ruvkun G. Caenorhabditis elegans Akt/PKB transduces insulin receptor-like signals from AGE-1 PI3 kinase to the DAF-16 transcription factor. Genes Dev. 1998;12(16):2488-98.

Passier R, Zeng H, Frey N, Naya FJ, Nicol RL, McKinsey TA, Overbeek P, Richardson JA, Grant SR, Olson EN .CaM kinase signaling induces cardiac hypertrophy and activates the MEF2 transcription factor in vivo. J Clin Invest 2000;105:1395-1406.

Peralta C, Perales JC, Bartrons R, Mitchell C, Gilgenkrantz H, Xaus C, Prats N, Fernández L, Gelpí E, Panés J, Roselló-Catafau J. The combination of ischemic preconditioning and liver $\mathrm{Bcl}-2$ overexpression is a suitable strategy to prevent liver and lung damage after hepatic ischemia-reperfusion. Am J Pathol. 2002;160(6):2111-22.

Piret JP, Minet E, Cosse JP, Ninane N, Debacq C, Raes M, Michiels C. Hypoxiainducible factor-1-dependent overexpression of myeloid cell factor-1 protects hypoxic cells against tert-butyl hydroperoxide-induced apoptosis. J Biol Chem. 2005;280(10):9336-44.

Plas DR, Thompson CB. Akt activation promotes degradation of tuberin and FOXO3a via the proteasome. J Biol Chem. 2003;278(14):12361-6. 
Prentice HM, Milton SL, Scheurle D, Lutz PL. The upregulation of cognate and inducible heat shock proteins in the anoxic turtle brain. J Cereb Blood Flow Metab 2004; 24(7):826-828.

Ramaglia V, Buck LT. Time-dependent expression of heat shock proteins 70 and 90 in tissues of the anoxic western painted turtle. J Exp Biol 2004; 207(Pt 21):377584.

Read MA, Brownell JE, Gladysheva TB, Hottelet M, Parent LA, Coggins MB, Pierce JW, Podust VN, Luo RS, Chau V, Palombella VJ. Nedd8 modification of cul-1 activates $\mathrm{SCF}($ beta(TrCP))-dependent ubiquitination of IkappaBalpha. Mol Cell Biol. 2000;20(7):2326-33.

Rena G, Prescott AR, Guo S, Cohen P, Unterman TG. Roles of the forkhead in rhabdomyosarcoma (FKHR) phosphorylation sites in regulating 14-3-3 binding, transactivation and nuclear targetting. Biochem J. 2001;354:605-612.

Rena G, Woods YL, Prescott AR, Peggie M, Unterman TG, Williams MR, Cohen P. Two novel phosphorylation sites on FKHR that are critical for its nuclear exclusion. EMBO J. 2002;21(9):2263-71.

Renna M, Caporaso MG, Bonatti S, Kaufman RJ, Remondelli P. Regulation of ERGIC53 gene transcription in response to endoplasmic reticulum stress. J Biol Chem. 2007;282(31):22499-512.

Reynisdóttir I, Polyak K, Iavarone A, Massagué J. Kip/Cip and Ink4 Cdk inhibitors cooperate to induce cell cycle arrest in response to TGF-beta. Genes Dev. 1995;9(15):1831-45.

Rice ME, Lee EJ, Choy Y. High levels of ascorbic acid, not glutathione, in the CNS of anoxia-tolerant reptiles contrasted with levels in anoxia-intolerant species. J Neurochem 1995; 64:1790-1799.

Ridder DA, Schwaninger M. NF-kappaB signaling in cerebral ischemia. Neuroscience. 2009;158(3):995-1006.

Rider MH, Hussain N, Dilworth SM, Storey KB. Phosphorylation of translation factors in response to anoxia in turtles, Trachemys scripta elegans: role of the AMPactivated protein kinase and target of rapamycin signalling pathways. Mol Cell Biochem. 2009;332(1-2):207-13.

Rojo AI, Salinas M, Martín D, Perona R, Cuadrado A. Regulation of Cu/Zn-superoxide dismutase expression via the phosphatidylinositol 3 kinase/Akt pathway and nuclear factor-kappaB. J Neurosci. 2004;24(33):7324-34.

Rolfe DF, Brown GC. Cellular energy utilization and molecular origin of standard metabolic rate in mammals. Physiol Rev 1997; 77:731-758. 
Ron D, Walter P. Signal integration in the endoplasmic reticulum unfolded protein response. Nat Rev Mol Cell Biol. 2007;8(7):519-29.

Ron D. Translational control in the endoplasmic reticulum stress response. J Clin Invest. 2002;110:1383-1388.

Ruddock LW, Molinari M. N-glycan processing in ER quality control. J Cell Sci. 2006;119(Pt 21):4373-80.

Rufo C, Teran-Garcia M, Nakamura MT, Koo SH, Towle HC, Clarke SD. Involvement of a unique carbohydrate-responsive factor in the glucose regulation of rat liver fatty-acid synthase gene transcription. J Biol Chem. 2001;276(24):21969-75.

Rupec RA, Baeuerle PA. The genomic response of tumor cells to hypoxia and reoxygenation. Differential activation of transcription factors AP-1 and NFkappa B. Eur J Biochem. 1995;234(2):632-40.

Rutkowski DT, Kaufman RJ. All roads lead to ATF4. Dev Cell. 2003;4(4):442-4.

Ruuge EK, Ledenev AN, Lakomkin VL, Konstantinov AA, Ksenzenko MYu. Free radical metabolites in myocardium during ischemia and reperfusion. Am J Physiol. 1991;261(4 Suppl):81-6.

Ryo A, Suizu F, Yoshida Y, Perrem K, Liou YC, Wulf G, Rottapel R, Yamaoka S, Lu KP. Regulation of NF-kappaB signaling by Pin1-dependent prolyl isomerization and ubiquitin-mediated proteolysis of p65/RelA. Mol Cell. 2003;12(6):1413-26.

Santore MT, McClintock DS, Lee VY, Budinger GR, and Chandel NS. Anoxia-induced apoptosis occurs through a mitochondria-dependent pathway in lung epithelial cells. Am J Physiol 2002; 282:L727-L734.

Sauve AA, Wolberger C, Schramm VL, Boeke JD. The biochemistry of sirtuins. Annu Rev Biochem.2006;75:435-465.

Scheuner D, Song B, McEwen E, Liu C, Laybutt R, Gillespie P, Saunders T, BonnerWeir S, Kaufman RJ. Translational control is required for the unfolded protein response and in vivo glucose homeostasis. Mol Cell. 2001;7(6):1165-76.

Schreck R, Rieber P, Baeuerle PA. Reactive oxygen intermediates as apparently widely used messengers in the activation of the NF-kappa B transcription factor and HIV-1. EMBO J. 1991;10(8):2247-58.

Schröder M, Kaufman RJ. ER stress and the unfolded protein response. Mutat Res. 2005a; 569(1-2):29-63.

Schröder M, Kaufman RJ. The mammalian unfolded protein response. Annu Rev Biochem. 2005b;74:739-89. 
Schröder M. Endoplasmic reticulum stress responses. Cell Mol Life Sci. 2008;65(6):86294

Schuetz A, Min J, Allali-Hassani A, Schapira M, Shuen M, Loppnau P, Mazitschek R, Kwiatkowski NP, Lewis TA, Maglathin RL, McLean TH, Bochkarev A, Plotnikov AN, Vedadi M, Arrowsmith CH. Human HDAC7 harbors a class IIa histone deacetylase-specific zinc binding motif and cryptic deacetylase activity. J Biol Chem. 2008; 283:11355-11363.

Scorziello A, Santillo M, Adornetto A, Dell'aversano C, Sirabella R, Damiano S, Canzoniero LM, Renzo GF, Annunziato L. NO-induced neuroprotection in ischemic preconditioning stimulates mitochondrial Mn-SOD activity and expression via Ras/ERK1/2 pathway. J Neurochem. 2007;103(4):1472-80.

Selye H. The nature of stress. Basal Facts. 1985;7(1):3-11.

Shen X, Ellis RE, Lee K, Liu CY, Yang K, Solomon A, Yoshida H, Morimoto R, Kurnit DM, Mori K, Kaufman RJ. Complementary signaling pathways regulate the unfolded protein response and are required for C. elegans development. Cell. 2001;107(7):893-903.

Shen X, Ellis RE, Sakaki K, Kaufman RJ. Genetic interactions due to constitutive and inducible gene regulation mediated by the unfolded protein response in $\mathrm{C}$. elegans. PLoS Genet. 2005;1(3):e37.

Sherr CJ, Roberts JM. CDK inhibitors: positive and negative regulators of G1-phase progression. Genes Dev. 1999; 13(12):1501-12.

Sherr CJ. Cancer cell cycles.Science. 1996;274(5293):1672-7.

Shi L, Wu J. Epigenetic regulation in mammalian preimplantation embryo development. Reprod Biol Endocrinol. 2009;7:59.

Shi Y, An J, Liang J, Hayes SE, Sandusky GE, Stramm LE, Yang NN. Characterization of a mutant pancreatic eIF-2alpha kinase; PEK, and co-localization with somatostatin in islet delta cells. J Biol Chem. 1999;274(9):5723-30.

Shi Y, Vattem KM, Sood R, An J, Liang J, Stramm L, Wek RC. Identification and characterization of pancreatic eukaryotic initiation factor 2 alpha-subunit kinase, PEK, involved in translational control. Mol Cell Biol. 1998; 18: 7499 7509.

Shih H, Towle HC. Definition of the carbohydrate response element of the rat S14 gene. Context of the CACGTG motif determines the specificity of carbohydrate regulation. J Biol Chem. 1994;269(12):9380-7. 
Sidrauski C, Walter $P$. The transmembrane kinase Irelp is a site-specific endonuclease that initiates mRNA splicing in the unfolded protein response. Cell. 1997;90(6):1031-9.

Smith EJ, Leone G, DeGregori J, Jakoi L, Nevins JR. The accumulation of an E2F-p130 transcriptional repressor distinguishes a G0 cell state from a G1 cell state. Mol Cell Biol. 1996; 16(12):6965-76.

Soares MP, Bach FH. Heme oxygenase-1: from biology to therapeutic potential. Trends Mol Me. 2009; 15(2):50-58.

Sobel RE, Cook RG, Perry CA, Annunziato AT, Allis CD. Conservation of depositionrelated acetylation sites in newly synthesized histones $\mathrm{H} 3$ and $\mathrm{H} 4$. Proc Natl Acad Sci USA. 1995; 92(4):1237-1241.

Spotswood HT, Turner BM. An increasingly complex code. J Clin Invest 2002; 110(5):577-582.

Sriburi R, Bommiasamy H, Buldak GL, Robbins GR, Frank M, Jackowski S, Brewer JW. Coordinate regulation of phospholipid biosynthesis and secretory pathway gene expression in XBP-1(S)-induced endoplasmic reticulum biogenesis. J Biol Chem. 2007;282(10):7024-34.

Stahl M, Dijkers PF, Kops GJ, Lens SM, Coffer PJ, Burgering BM, Medema RH. The forkhead transcription factor FoxO regulates transcription of $\mathrm{p} 27 \mathrm{Kip} 1$ and Bim in response to IL-2. J Immunol. 2002;168(10):5024-31.

Stokoe D, Stephens LR, Copeland T, Gaffney PR, Reese CB, Painter GF, Holmes AB, McCormick F, Hawkins PT. Dual role of phosphatidylinositol-3,4,5trisphosphate in the activation of protein kinase B. Science. 1997;277(5325):567-70.

Storey KB, Storey JM. Metabolic rate depression in animals: transcriptional and translational controls. Biol Rev Camb Philos Soc 2004; 79(1):207-233.

Storey KB, Storey JM. Putting life on 'pause' - molecular regulation of hypometabolism. J Exp Biol 2007; 210(Pt 10):1700-1714.

Storey KB. Anoxia tolerance in turtles: metabolic regulation and gene expression. Comp Biochem Physiol A 2007; 147(2):263-276.

Storey KB. Gene hunting in hypoxia and exercise. Adv Exp Biol Med 2006;588:293-309.

Storey KB. Metabolic adaptations supporting anoxia tolerance in reptiles: recent advances. Comp Biochem Physiol B 1996;113(1):23-35. 
Storey, KB. Oxygen limitation and metabolic rate depression, in: K.B. Storey (Ed.), Functional Metabolism: Regulation and Adaptation. Hoboken, NJ: Wiley-Liss, 2004a: 415-442.

Strahl BD, Allis CD (2000) The language of covalent histone modifications. Nature 403:41-45.

Sun XJ, Rothenberg P, Kahn CR, Backer JM, Araki E, Wilden PA, Cahill DA, Goldstein BJ, White MF. Structure of the insulin receptor substrate IRS-1 defines a unique signal transduction protein. Nature. 1991;352(6330):73-7.

Tacchini L, Recalcati S, Bernelli-Zazzera A, Cairo G. Induction of ferritin synthesis in ischemic-reperfused rat liver: analysis of the molecular mechanisms. Gastroenterology. 1997;113(3):946-53.

Tanaka M, Kirito K, Kashii Y, Uchida M, Watanabe T, Endo H, Endoh T, Sawada K, Ozawa K, Komatsu N. Forkhead family transcription factor FKHRL1 is expressed in human megakaryocytes. Regulation of cell cycling as a downstream molecule of thrombopoietin signaling. J Biol Chem. 2001;276(18):15082-9.

Tattersall GJ, Boutilier RG. Balancing hypoxia and hypothermia in cold-submerged frogs. J Exp Biol. 1997;200(Pt 6):1031-8.

Towle HC. Glucose as a regulator of eukaryotic gene transcription. Trends Endocrinol Metab. 2005;16(10):489-94.

Tran H, Brunet A, Grenier JM, Datta SR, Fornace AJ Jr, DiStefano PS, Chiang LW, Greenberg ME. DNA repair pathway stimulated by the forkhead transcription factor FOXO3a through the Gadd45 protein. Science. 2002;296(5567):530-4.

Travers, K. J., Patil, C. K., Wodicka, L., Lockhart, D. J., Weissman, J. S. andWalter, P. Functional and genomic analyses reveal an essential coordination between the unfolded protein response and ER-associated degradation. Cell. 2000; 101:249-258.

Ultsch GR. The ecology of overwintering among turtles: where turtles overwinter and its consequences. Biol Rev Camb Philos Soc 2006; 81(3):339-367.

Valko M, Izakovic M, Mazur M, Rhodes CJ, Telser J. Role of oxygen radicals in DNA damage and cancer incidence. Mol Cell Biochem 2004; 266(1-2):37-56.

Van Breukelen F, Maier R, Hand SC. Depression of nuclear transcription and extension of mRNA half-life under anoxia in Artemia franciscana embryos. J Exp Biol 2000; 203:1123-1130.

Van Breukelen F, Martin SL. Reversible depression of transcription during hibernation. J Comp Physiol B 2002; 172:355-361. 
van der Horst A, Burgering BM. Stressing the role of FoxO proteins in lifespan and disease. Nat Rev Mol Cell Biol. 2007;8(6):440-50.

van der Horst A, de Vries-Smits AM, Brenkman AB, van Triest MH, van den Broek N, Colland F, Maurice MM, Burgering BM. FOXO4 transcriptional activity is regulated by monoubiquitination and USP7/HAUSP. Nat Cell Biol. 2006;8(10):1064-73.

van der Maarel SM. Epigenetic mechanisms in health and disease. Ann Rheum Dis.2008; 67 Suppl 3:iii97-100.

van der Vos KE, Coffer PJ. FOXO-binding partners: it takes two to tango. Oncogene. 2008;27:2289- 2299.

Vega RB, Harrison BC, Meadows E, Roberts CR, Papst PJ, Olson EN, McKinsey TA Protein kinases $\mathrm{C}$ and $\mathrm{D}$ mediate agonist-dependent cardiac hypertrophy through nuclear export of histone deacetylase 5. Mol Cell Biol. 2004;24:83748385.

Verdin E, Dequiedt F, Kasler HG. Class II histone deacetylases: versatile regulators. Trends Genet. 2003;19:286-293.

Vig E, Nemcsok J. The effects of hypoxia and paraquat on the superoxide dismutase activity in different organs of carp Cyprinus carpio L. J Fish Biol. 1989;35:2325.

Vile GF, Basu-Modak S, Waltner C, Tyrrell RM. Heme oxygenase 1 mediates an adaptive response to oxidative stress in human skin fibroblasts. Proc Natl Acad Sci USA 1994; 91(7):2607-2610.

Wade PA.Transcriptional control at regulatory checkpoints by histone deacetylases: molecular connections between cancer and chromatin. Hum Mol Genet. 2001; 10:693-698

Warburton SJ, Jackson DC. Turtle (Chrysemys picta bellii) shell mineral content is altered by exposure to prolonged anoxia. Physiol Zool. 1995; 68:783-798.

Wasser JS, Warburton SJ, Jackson DC. Extracellular and intracellular acid-base effects of submergence anoxia and nitrogen breathing in turtles. Resp Physiol. 1991; $83: 239-252$

Watowich SS, Morimoto RI, Lamb RA. Flux of the paramyxovirus hemagglutininneuraminidase glycoprotein through the endoplasmic reticulum activates transcription of the GRP78-BiP gene. J Virol. 1991;65(7):3590-7.

Wek RC, Cavener DR. Translational control and the unfolded protein response. Antioxid Redox Signal. 2007;9(12):2357-71. 
Willmore WG, Storey KB. Antioxidant systems and anoxia tolerance in a freshwater turtle Trachemys scripta elegans. Mol Cell Biochem. 1997a; 170:177-185.

Willmore WG, Storey KB. Glutathione systems and anoxia tolerance in turtles. Am J Physiol. 1997b; 273:R219-R225.

Willmore WG, Storey KB. Purification and properties of glutathione reductase from liver of the anoxia-tolerant turtle, Trachemys scripta elegans. Mol Cell Biochem. 2007; 297(1-2):139-149.

Willmore WG, Storey KB. Purification and properties of the glutathione S-transferases from the anoxia-tolerant turtle, Trachemys scripta elegans. FEBS J. 2005; 272(14):3602-3614.

Winter J, Jakob U. Beyond transcription - new mechanisms for the regulation of molecular chaperones. Crit Rev Biochem Mol Biol. 2004; 39:297-317.

Wolffe AP, Matzke MA. Epigenetics: regulation through repression. Science. 1999;286:481-486.

Wu S, Tan M, Hu Y, Wang JL, Scheuner D, Kaufman RJ. Ultraviolet light activates NFkappaB through translational inhibition of IkappaBalpha synthesis. J Biol Chem. 2004;279(33):34898-902.

Yagi A, Hasegawa Y, Xiao H, Haneda M, Kojima E, Nishikimi A, Hasegawa T, Shimokata K, Isobe K. GADD34 induces p53 phosphorylation and p21/WAF1 transcription. J Cell Biochem. 2003;90(6):1242-9.

Yamada K, Noguchi T. Nutrient and hormonal regulation of pyruvate kinase gene expression. Biochem J. 1999;337 (Pt 1):1-11.

Yamamoto K, Yoshida H, Kokame K, Kaufman RJ, Mori K. Differential contributions of ATF6 and XBP1 to the activation of endoplasmic reticulum stress-responsive cis-acting elements ERSE, UPRE and ERSE-II. J Biochem. 2004;136(3):34350.

Yamashita H, Takenoshita M, Sakurai M, Bruick RK, Henzel WJ, Shillinglaw W, Arnot $\mathrm{D}$, Uyeda K. A glucose-responsive transcription factor that regulates carbohydrate metabolism in the liver. Proc Natl Acad Sci U S A. 2001;98(16):9116-21.

Yamashita N, Nishida M, Hoshida S, Kuzuya T, Hori M, Taniguchi N, Kamada T, Tada $M$. Induction of manganese superoxide dismutase in rat cardiac myocytes increases tolerance to hypoxia 24 hours after preconditioning. J Clin Invest. 1994;94(6):2193-9. 
Yang H, Park SH, Choi HJ, Moon Y. Epithelial cell survival by activating transcription factor 3 (ATF3) in response to chemical ribosome-inactivating stress. Biochem Pharmacol. 2009;77(6):1105-15.

Yoshida H, Okada T, Haze K, Yanagi H, Yura T, Negishi M, Mori K. ATF6 activated by proteolysis binds in the presence of NF-Y (CBF) directly to the cis-acting element responsible for the mammalian unfolded protein response. Mol Cell Biol. 2000;20(18):6755-67.

Yoshida H, Matsui T, Yamamoto A, Okada T, Mori K. XBP1 mRNA is induced by ATF6 ánd spliced by IRE1 in response to ER stress to produce a highly active transcription factor. Cell. 2001;107:881-891

Yoshida, H., Matsui, T., Hosokawa, N., Kaufman, R. J., Nagata, K. and Mori, K. A timedependent phase shift in the mammalian unfolded protein response. Dev Cell. $2003 ; 4: 265-271$.

Zhan L, Wang T, Li W, Xu ZC, Sun W, Xu E. Activation of Akt/FoxO signaling pathway contributes to induction of neuroprotection against transient global cerebral ischemia by hypoxic preconditioning in adult rats. J Neurochem. 2010 Epub May 18.

Zhang CL, McKinsey TA, Lu JR, Olson EN. Association of COOH-terminal-binding protein (CtBP) and MEF2-interacting transcription repressor (MITR) contributes to transcriptional repression of the MEF2 transcription factor. $\mathbf{J}$ Biol Chem. 2001;276:35-39.

Zhang X, Gan L, Pan H, Guo S, He X, Olson ST, Mesecar A, Adam S, Unterman TG. Phosphorylation of serine 256 suppresses transactivation by FKHR (FOXO1) by multiple mechanisms. Direct and indirect effects on nuclear/cytoplasmic shuttling and DNA binding. J Biol Chem. 2002a;277(47):45276-84.

Zhang CL, McKinsey TA, Olson EN. Association of class II histone deacetylases with heterochromatin protein 1: potential role for histone methylation in control of muscle differentiation. Mol Cell Biol. 2002b;22:7302-7312.

Zong WX, Edelstein LC, Chen C, Bash J, Gélinas C. The prosurvival Bcl-2 homolog Bfl1/A1 is a direct transcriptional target of NF-kappaB that blocks TNFalphainduced apoptosis. Genes Dev. 1999;13(4):382-7. 